SAND96-2736 • UC-721

\title{
Automation of ORIGEN2 Calculations for the Transuranic Waste Baseline Inventory Database Using a Pre-Processor and a Post-Processor
}

Jennifer LiscumHPowell

Prepared by

Sandia National Laboratorios

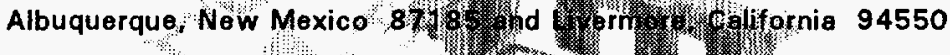

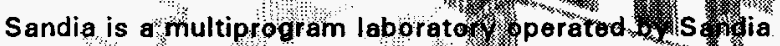

Corporation, a Lockteed Martin O. Jpany, for the United States

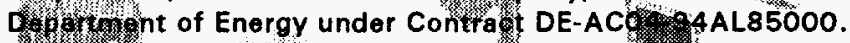

(1)

(Y)

Approved for public release; distribution is unlimif

ution is unlimit

(10) Sandia National Labogerories
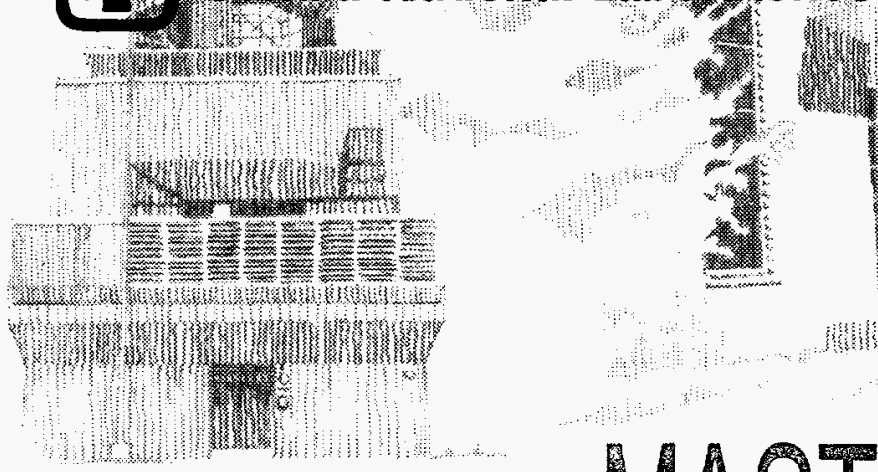

SF $29000(8-81)$
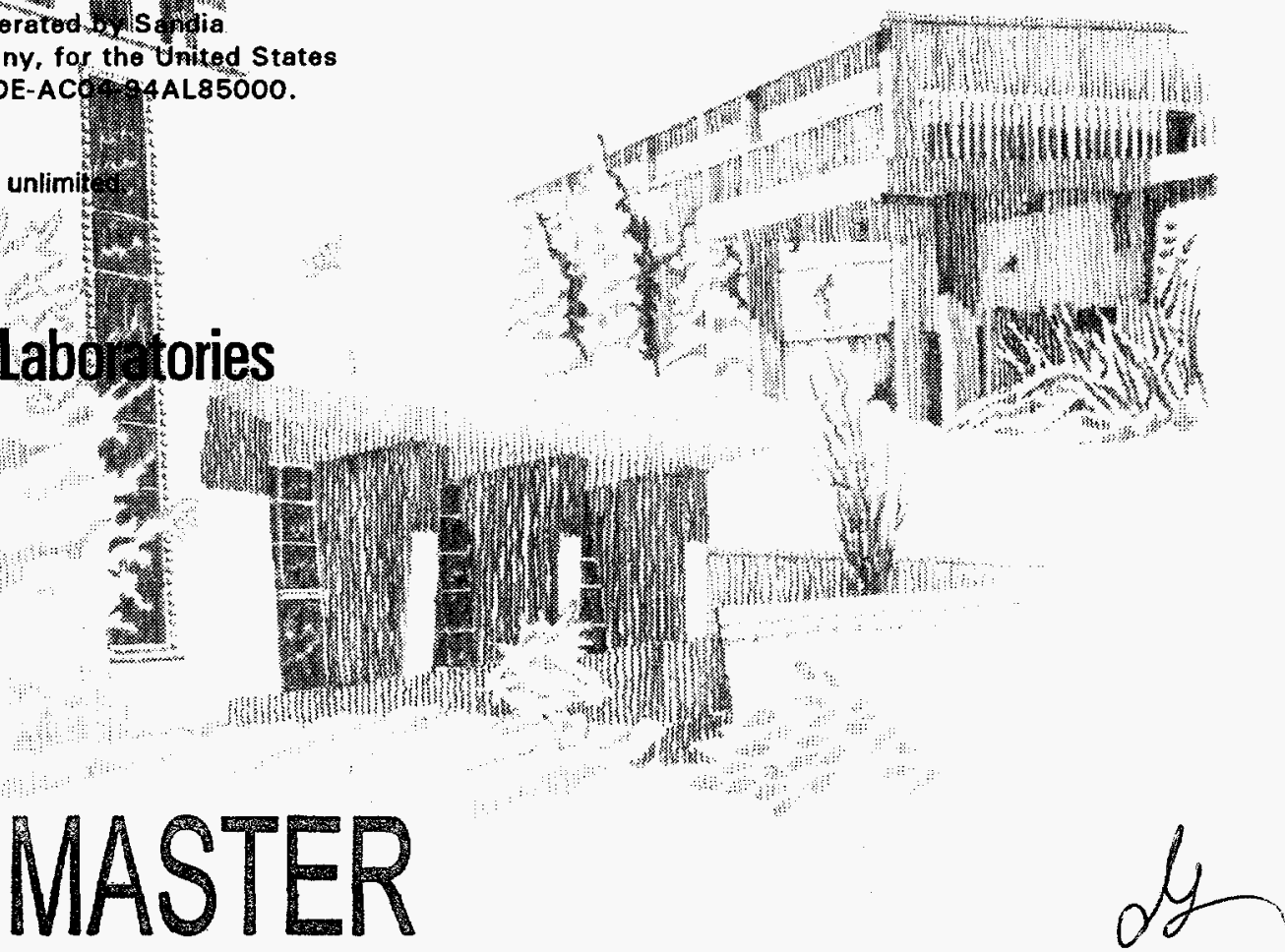

DSTRIBUTION OF THIS DOCUMENT IS UNLIMITED 
Issued by Sandia National Laboratories, operated for the United States Department of Energy by Sandia Corporation.

NOTICE: This report was prepared as an account of work sponsored by an agency of the United States Government. Neither the United States Government nor any agency thereof, nor any of their employees, nor any of their contractors, subcontractors, or their employees, makes any warranty, express or implied, or assumes any legal liability or responsibility for the accuracy, completeness, or usefulness of any information, apparatus, product, or process disclosed, or represents that its use would not infringe privately owned rights. Reference herein to any specific commercial product, process, or service by trade name, trademark, manufacturer, or otherwise, does not necessarily constitute or imply its endorsement, recommendation, or favoring by the United States Government, any agency thereof, or any of their contractors or subcontractors. The views and opinions expressed herein do not necessarily state or reflect those of the United States Government, any agency thereof, or any of their contractors.

Printed in the United States of America. This report has been reproduced directly from the best available copy.

Available to DOE and DOE contractors from

Office of Scientific and Technical Information

P.O. Box 62

Oak Ridge, TN 37831

Prices available from (615) 576-8401, FTS 626-8401

Available to the public from

National Technical Information Service

U.S. Department of Commerce

5285 Port Royal Rd

Springfield, VA 22161

NTIS price codes

Printed copy: A08

Microfiche copy: A01 


\title{
Automation of ORIGEN2 Calculations for the Transuranic Waste Baseline Inventory Database Using a Pre-Processor and a Post-Processor
}

\author{
Jennifer Liscum-Powell \\ Nuclear Safety and Systems Analysis \\ Sandia National Laboratories \\ P.O. Box 5800 \\ Albuquerque, NM 87185-1175
}

\begin{abstract}
The purpose of the work described in this report was to automate ORIGEN2 calculations for the Waste Isolation Pilot Plant (WIPP) Transuranic Waste Baseline Inventory Database (WTWBID); this was done by developing a pre-processor to generate ORIGEN2 input files from WWBID inventory files and a post-processor to remove excess information from the ORIGEN2 output files. The calculations performed with ORIGEN2 estimate the radioactive decay and buildup of various radionuclides in the waste streams identified in the WTWBD. The resulting radionuclide inventories are needed for performance assessment calculations for the WIPP site.

The work resulted in the development of PreORG, which requires interaction with the user to generate ORIGEN2 input files on a site-by-site basis, and PostORG, which processes ORIGEN2 output into more manageable files. Both programs are written in the FORTRAN 77 computer language. After running PreORG, the user will run ORIGEN2 to generate the desired data; upon completion of ORIGEN2 calculations, the user can run PostORG to process the output to make it more manageable. All the programs run on a $386 \mathrm{PC}$ or higher with a math co-processor or a computer platform running under VMS operating system. The pre- and post-processors for ORIGEN2 were generated for use with Rev. 1 data of the WTWBID and can also be used with Rev. 2 and 3 data of the TWBID (Transuranic Waste Baseline Inventory Database).
\end{abstract}




\section{ACKNOWLEDGMENTS}

The author wishes to thank Lawrence Sanchez, Org. 6848, and Leo Rahal,

Geocenters Inc., for their technical review and comments regarding this document. The author also wishes to thank Molly Minahan, Tech Reps, Inc., for her assistance as a technical editor and writer, and Debbie Rivard and Jackie Ripple, Tech Reps Inc., for their expert word processing. Carol Crawford, 4415 , provided a comprehensive reference check of this document. 


\section{CONTENTS}

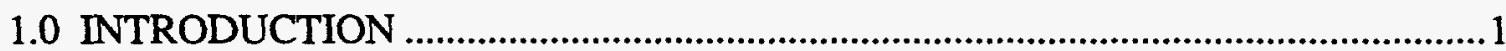

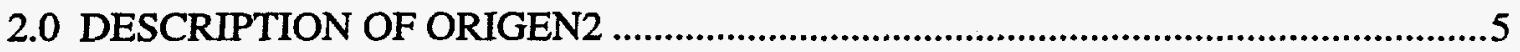

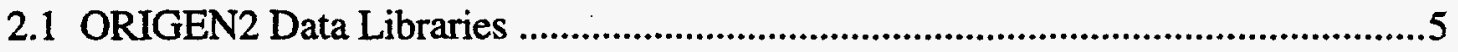

2.2 Characteristics ORIGEN2 Calculates .................................................................6

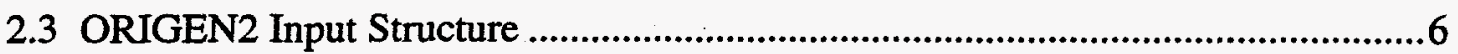

2.3.1 Entering Initial Nuclide Concentrations ...................................................

2.4 Calculations Performed by ORIGEN2 ..............................................................

2.5 Method of Solving Differential Equations in ORIGEN2 .......................................6

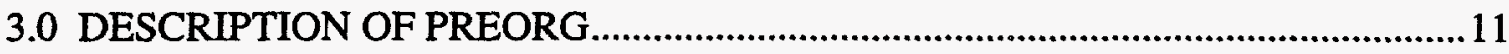

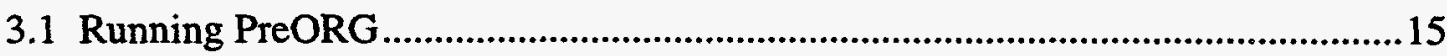

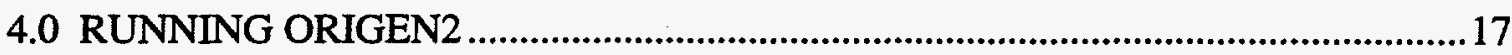

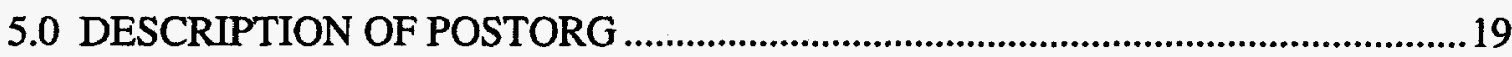

5.1 Running PostORG

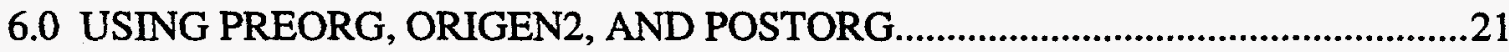

6.1 Running PreORG, ORIGEN2, and PostORG on a PC ........................................21

6.2 Running PREORG, ORIGEN2, and POSTORG on a VMC computer platform.22

6.3 Cautions to Observe when Running PreORG, ORIGEN2, and PostORG ............22

7.0 SUMMARY

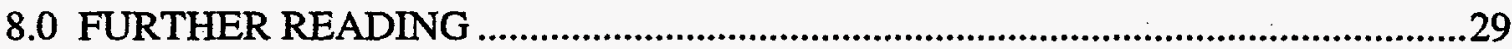

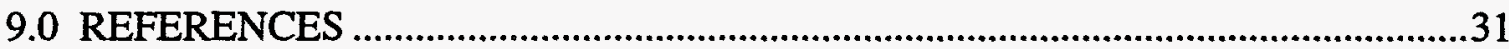

APPENDIX A： SAMPLE PREORG.FOR DATA INPUT FILE ....................................A-1

APPENDIX B: ORIGEN2.BAT FILE LISTING FOR A PC ........................................1

APPENDIX C: ORIGEN2 COMMAND DESCRIPTIONS AND SAMPLE ORIGEN2 INPUT FILE CREATED BY PREORG .............................................

APPENDIX D： OR1_PREORG.FOR PROGRAM LISTING_.....................................

APPENDIX E: PERSONAL COMMUNICATION WITH SAYAN CHAKRABORTI OF SCIENCE APPLICATIONS INTERNATIONAL CORPORATION BY JENNIFER POWELL ON JULY 26, 1995 ..................................E-1

APPENDIX F: $\quad$ OR3_POSTORG.FOR PROGRAM LISTING ...................................... F-1 
Figures

Figure 6.2.1 Sample command file used to run PreORG, ORIGEN2, and PostORG. .....23

\section{Tables}

Table 1. ORIGEN2 Input Files Created by PreORG 


\section{DISCLAMIXR}

Portions of this document may be illegible in electronic image products. Images are produced from the best available original document. 


\subsection{INTRODUCTION}

The purpose of this work was to automate ORIGEN2 calculations for the Waste Isolation Pilot Plant (WIPP) Transuranic Waste Baseline Inventory Database (WTWBID). This automation was accomplished by developing a pre-processor that generates complete ORIGEN2 input files from Baseline Inventory Database (BID) data

files and a post-processor that processes the ORIGEN2 output files by extracting only the useful information.

There are two printed versions of the radionuclide inventory data at the present time. The first version is the WIPP Transurance Waste Baseline Inventory Report (WTWBIR), which is the WTWBID (Rev. 1 of radionuclide data) (WTAC, 1995a). The second version is the Transurance Waste Baseline Inventory Report (TWBIR), which is in TWBID (Rev. 2 of the radionuclide data) (WTAC, 1995b). PREORG and POSTORG can be used with both sets of data.

The calculations performed with ORIGEN2 estimate the radioactive decay and buildup of various radionuclides in the WIPP waste streams identified in the WTWBID. The resulting radionuclide inventories are needed for performance assessment calculations for the WIPP site.

For the purposes of the WIPP Performance Assessment (PA), PreORG and PostORG are used in conjunction with ORIGEN2 to perform decay and radioactive buildup calculations for inventories of "stored" waste (also termed legacy waste). The data for the decayed stored waste is used directly by the TWBIR team. The TWBIR team supplements this data with information on "future" or projected waste to yield WIPPScale data, which is used directly in the WIPP PA calculations as part of the source term parameters (i.e., these data are incorporated into the WIPP PA database).

The work resulted in the development of PreORG, which requires interaction with the user to generate ORIGEN2 input files on a site-by-site basis, and PostORG, which processes ORIGEN2 output into more manageable files. Both programs are written in the FORTRAN 77 computer language. After running PreORG, the user will run ORIGEN2 
to generate the desired data; upon completion of ORIGEN2 calculations, the user can run PostORG to process the output to make it more manageable. All the programs run on a $386 \mathrm{PC}$ or higher with a math co-processor or a computer platform running under a VMS operating system.

PostORG and PreORG meet the requirements of the WIPP Quality Assurance Plan with extensive testing to ensure accuracy of the calculations performed by this suite of codes (PreORG, ORIGEN2, and.PostORG). Documentation of PreORG and PostORG compliance with the requirements of the WIPP Performance Assessment Quality Assurance Plan is located in the WIPP PA Analysis Report for ORIGEN2 Suite (LiscumPowell et al., 1996). PreORG and PostORG were tested against analytical and hand calculations to verify the accuracy and validity of results obtained from both of these codes (Liscum-Powell et al., 1996).

To utilize this program, data from the WTWBID must be located in data files structured as depicted in Appendix A. Each site (i.e., waste generating site that sends TRU waste to the WIPP facility) has its own data file for each type of waste: contacthandled transuranic (CH-TRU), remotely handled (RH-TRU), and buried TRU. Using the pre-processor system, the user enters the site of interest as well as the type of waste (CH-TRU, RH-TRU, or BURIED TRU) of interest and a base year; the purpose of this process is to decay all inventory in the associated data files to a specific starting point for further decay calculations. The purpose of the base year is to bring all the data for a given site to the same point in time, as site data files list nuclide inventories year by year (e.g., $1970,1971,1973$, etc.). Following completion of calculations to decay all data to the designated year, PostORG can be used to generate an additional ORIGEN2 input file to decay the total base year inventory for a site into the future. PostORG allows the user to choose how far into the future he/she would like to decay the base year inventory, ranging from 75 years to 10,000 years. (The user should note that ORIGEN2 input files generated by PreORG and PostORG can be used as a template to assist in developing input files for other ORIGEN2 problems, by making the appropriate modifications to the cards. A card 
is a line of data in the ORIGEN2 input file that includes an ORIGEN2 command and the information needed to execute the command.)

This report provides documentation on the programs developed in the course of this work as well as a description of the ORIGEN2 code. When using PreORG and PostORG, this report can be used as a manual to aid in performing the calculations desired. This report describes the three codes: ORIGEN2 (Section 2), PreORG (Section 3), and PostORG (Section 5). Section 4 details how to run ORIGEN2. Section 6 provides step-by-step instructions on using PreORG, ORIGEN2, and PostORG. A summary is presented in Section 7 and references are provided in Section 8. 
Intentionally Left Blank 


\subsection{DESCRIPTION OF ORIGEN2}

ORIGEN2 is a point-depletion and radioactive-decay computer code developed at Oak Ridge National Laboratory (ORNL) and distributed by the Laboratory's Radiation Shielding Information Center (RSIC). This code was developed to simulate nuclear fuel cycles and calculate nuclide compositions and characteristics of materials. One of its primary uses is to evaluate the radionuclide composition of nuclear waste material following its radioactive decay. The code itself is written entirely in the FORTRAN computer language and runs in a matter of minutes on a 386 PC (see Appendix B) or higher with a math co-processor (Croff, 1980).

\subsection{ORIGEN2 Data Libraries}

The ORIGEN2 database consists of three libraries: (1) activation products with 720 nuclides, (2) actinides with 130 nuclides, and (3) fission products with 850 nuclides (Croff, 1980). Of the nuclides in the database, 1300 are unique isotopes; 300 of these nuclides are stable. When running ORIGEN2, it is necessary that all nuclides from a given library are grouped together - starting first with the activation products, followed by the actinides and fission products, respectively (see Appendix C). The decay library contains these three libraries in three separate sections with two cards for each nuclide. The information contained for each nuclide includes the nuclide identifier, radioactive half-life, type of decay, and information on build-up of the nuclide. The nuclide identifier used by ORIGEN2 for a given nuclide is determined as follows (Croff, 1980):

$$
\text { NUCLID }=10000 * Z+10 * A+I S
$$

where NUCLID is the six-digit nuclide identifier used in the code, $\mathrm{Z}$ is the nuclide atomic number, $\mathrm{A}$ is the atomic mass of nuclide, and IS indicates the isomeric state of the nuclide $(0=$ ground state or $1=$ excited state $)$. For example, the nuclide identifier for Pu239 ( $\mathrm{Z}=94, \mathrm{~A}=239$ ) would be 942390 . 


\subsection{Characteristics ORIGEN2 Calculates}

ORIGEN2 can determine numerous characteristics of the nuclear materials following radioactive buildup and decay of a material and can simulate the reactor irradiation of cladding and structural material and burnup of the fuel following simulation of reactor operation. These characteristics include the following: mass, fractional isotopic composition, radioactivity, thermal power, toxicity following ingestion or inhalation, neutron absorption and emission rates, and photon emission rates (Croff, 1980). Internally, ORIGEN2 stores these data or characteristics in tables or vectors indexed by user-assigned numbers. By including certain commands in the input file, the user controls what type of data ORIGEN2 will generate. Appendix C contains a sample ORIGEN2 input file generated by PreORG; Appendix $\mathrm{C}$ also provides tables indicating the type of data the code will generate and information regarding how to instruct ORIGEN2 to include particular information in its output files.

\subsection{ORIGEN2 Input Structure}

The ORIGEN2 input structure is composed of a series of command cards with each line of input representing a single operation and the details of its execution. The commands are executed sequentially by ORIGEN2 as they are read from the input file. Every ORIGEN2 input file must have the extension.U5 to indicate to the code that the input file is the source of ORIGEN2's instructions for a given run. To perform a simple radioactive decay calculation as is done in this work, the following instructions must be given to ORIGEN2 in the input file:

1. Indicate the data libraries to be used for the calculations.

2. Instruct ORIGEN2 to read initial radionuclide concentrations in grams or gramsatom. (Note: ORIGEN2 does not accept input in Curies although it will output values in Curies. When converting curies into grams for ORIGEN2 input, the user should use the half lives from the ORIGEN2 decay library. The ORIGEN2 decay library is a part of the ORIGEN2 code package and can be found in the directory where the data libraries are located [e.g., /ORIGEN2/LIBS]).

3. Instruct ORIGEN2 to decay out initial data for a given length of time:

4. Tell ORIGEN2 what output tables you desire. 
5. Instruct ORIGEN2 to write desired results to the output file. (Note: The primary ORIGEN2 output files have the extension .U6).

6. Terminate execution of program.

The steps above are accomplished using a series of commands as those shown in the sample input file in Appendix C. It should be noted that there are several limitations in ORIGEN2 as far as the number of command cards of a certain type that can be used; the user is directed to the ORIGEN2 manual for further elaboration (Croff, 1980). For instance, the total number of commands that can be used in a given input file is 300 .

\subsubsection{Entering Initial Nuclide Concentrations}

The initial nuclide data is either entered in the ORIGEN2 input file after the STP or END card, or it is placed in an external file with the proper instruction. Each line of initial data cannot exceed 80 bytes (equal to 3 nuclide-identifier and initial-concentration pairs) and must end with two zeros $(00)$ to indicate that the end of that card has been reached. The first entry on a card must indicate which of the three libraries the nuclidedecay and accumulation data is contained in $(1,2$, or 3$)$; all nuclides from a given library must be grouped together. Each input card reads data until it encounters a line with one zero (0) indicating that the following data is read by a new input card. A particular isotope can be read in a given input string more than once and ORIGEN2 will calculate internally the total initial concentration of the repeated nuclide.

\subsection{Calculations Performed by ORIGEN2}

ORIGEN2 performs a number of trivial calculations such as simple summations and multiplications, but it is also characterized by two unique features (Croff, 1983): (1) the method it employs to store the equations describing radionuclide decay and accumulation and (2) the method employed to solve these equations for nuclide concentrations. The general form of the equation ORIGEN2 solves is as follows (Croff, 1983): 


$$
\frac{d N_{i}}{d t}=\sum_{j=1}^{N_{\text {tot }}} \lambda_{i j} \lambda_{j} N_{j}+\phi \sum_{k=1}^{N_{\text {tot }}} f_{i k} \sigma_{k} N_{k}-\left(\lambda_{i}+\phi \sigma_{i}+r_{i}\right) N_{i}+F_{i}, i=1, \ldots, \mathrm{N}_{\mathrm{tot}}
$$

where

$$
\begin{aligned}
\mathrm{N}_{i}, \mathrm{~N}_{k}= & \text { Atom density of nuclide } i, k \\
\mathrm{~N}_{\text {tot }}= & \text { Number of nuclides } \\
\lambda_{i j}= & \text { Fraction of radioactive disintegration by other nuclides that leads to } \\
& \text { formation of species } i \\
\lambda_{i}= & \text { Radioactive decay constant of species } i \\
\phi= & \text { Position and energy averaged neutron flux } \\
\sigma_{k}= & \text { Spectrum averaged neutron absorption cross - section of nuclide } k \\
\mathrm{f}_{i k}= & \text { Fraction of neutron absorption by other nuclides that leads to formation } \\
& \text { of species } i \\
\mathrm{r}_{i}= & \text { Continuous removal rate of nuclide } i \text { from the system } \\
\mathrm{F}_{i}= & \text { Continuous feed rate of nuclide } i
\end{aligned}
$$

For each nuclide of interest, there is one equation of this general form. For decay calculations independent of constant power or flux irradiation, these equations reduce to the Bateman equations (Kaplan, 1964):

$$
\frac{d N_{i}}{d t}=\sum_{j=1}^{N_{t o t}} \lambda_{i j} \lambda_{j} N_{j}-\lambda_{i} N_{i}, i=1, \ldots, \mathrm{N}_{\text {tot }}
$$

These equations make up a system of ordinary differential equations that translate into a sparse matrix. ORIGEN2 stores only the nonzero elements of this matrix by utilizing indexing techniques in what is referred to as the transition matrix (Croff, 1983). Thus, the transition matrix contains all the transformation rates of each parent nuclide through decay and other processes such as neutron absorption. 


\subsection{Method of Solving Differential Equations in ORIGEN2}

The coupled, ordinary differential equations discussed above that represent the accumulation and decay of each nuclide of interest in an ORIGEN2 calculation are solved internally using a compilation of three solution techniques (Croff, 1983). The primary or cornerstone solution technique is the exponential matrix technique; however, problems are encountered with this method when trying to solve a matrix containing widely separated eigenvalues, which are often encountered with the type of problems ORIGEN2 solves (Croff, 1983). To avoid these calculation problems, asymptotic versions of analytical solutions to the nuclide accumulation and decay equations are employed at different points during the solution process (Croff, 1983).

Essentially, ORIGEN2 solves the system of ordinary differential equations by following three steps for each time step or decay interval. These solution steps include the following (Croff, 1983):

1. A set of asymptotic solutions are utilized that are suitable for handling short-lived nuclides whose parent nuclides are not long-lived. ORIGEN2 defines a shortlived nuclide as one whose half-life is less than $14.4 \%$ that of the decay interval (Croff, 1983). The concentrations of these nuclides reach an equilibrium or constant value within the time step, and thus, the asymptotic solution is an adequate approximation. These solutions are used to quantify nuclides that reach transient equilibrium during a given decay interval. Transient equilibrium occurs when the parent nuclide is characterized by a half-life that is greater than its daughter's half-life but not by an excessive amount $\left(T_{1 / 2}^{P} \approx 3\right.$ to 10 times $\left.T_{1 / 2}^{D}\right)$.

2. The transition matrix is further reduced to a matrix containing only long-lived nuclides and this reduced matrix is solved for the concentrations of these nuclides at the end of the time step using the exponential method. The matrix exponential method utilizes the series representation of the exponential function by translating the summations found in Equations (1) and (2) into exponential functions. The exponential method is described by the equations below (Croff, 1983):

$$
\mathbf{N}_{\mathbf{d t}}=\mathbf{A N}
$$


where $\mathbf{N}_{\mathbf{d t}}$ is a column matrix with the time derivative of the nuclide concentrations, $\mathbf{A}$ is the reduced transition matrix with the long-lived nuclide transformation rates, and $\mathbf{N}$ is a column matrix containing the nuclide concentrations at the end of the time step. Using the exponential method solution technique, this equation reduces to the following relationship (Croff, 1983):

$$
\mathrm{N}(t)=\mathrm{e}^{\mathrm{At}} \mathrm{N}(t=0)
$$

where $\mathrm{N}(t)$ is the nuclide concentration at time $t, \mathrm{~N}(t=0)$ is the initial nuclide concentration at the beginning of the decay interval, and $t$ is the time at the end of a given decay interval.

3. During the third and final calculation step, ORIGEN2 uses another set of asymptotic soluticns to analytical equations to calculate concentrations of shortlived nuclides with long-lived parents $\left(T_{1 / 2}^{P}>10\right.$ times $\left.T_{1 / 2}^{D}\right)$. These nuclides reach secular equilibrium in which the daughter and parent activity reach an equilibrium state after approximately seven daughter half-lives have passed. The Gauss-Seidel successive substitution method is employed to solve these equations (Croff, 1983). 


\subsection{DESCRIPTION OF PREORG}

The program PreORG was developed to serve as a pre-processor to ORIGEN2 calculations by automating WIPP TWBID data files into ORIGEN2 input files to perform nuclide decay and accumulation calculations. A listing of PreORG, written entirely in the FORTRAN computer language, is located in Appendix D.

The pre-processor requires interaction with the user and generates ORIGEN2 input files through the following steps:

STEP 1 Prompts user for input:

a. Base year to which all data are initially decayed

b. Filename to write a list of input files generated

c. Site name

d. Enriched Uranium isotopic composition

e. Type of waste

STEP 2 Reads all data from input file into arrays containing nuclide name (e.g., PU239) and the initial activity in Curies. Counts the number of years in the data file and the number of isotopes in each year.

STEP 3 Writes basic ORIGEN2 commands to read input for each year.

STEP 4 Writes ORIGEN2 commands to decay all data to the common base year given by user.

STEP 5 Converts all nuclide activities to grams using specific activities, assigns appropriate nuclide identifiers (e.g., PU239 $\Rightarrow 942390$ ), and determines ORIGEN2 data library where each nuclide decay data is located. (Note: Halflives used to determine a nuclide activity are identical to those found in the ORIGEN2 decay libraries.)

STEP 6 Writes out initial activities year-by-year, one library at a time (Appendix B).

STEP 7 Ensures all data in the PreORG input file has been incorporated in ORIGEN2 input file (PreORG output file). Because each ORIGEN2 input file can use a maximum of 15 input cards, if there are more than 15 years of data, return to STEP 3 using STP card and repeat STEPS 3 to 6 until all data are in input file.

STEP 8 Prompts user to indicate whether or not he/she would like to generate more input files (for a given run, PreORG assumes same base year for all sites):

- If yes, repeat STEPS 1(c) through 1(e) and STEPS 2 through 7. 
- If no, terminate execution.

Appendix A contains a list of input files that PreORG will accept from the WIPP WTWBID, plus a listing of what is written to the screen when running PreORG with examples of user input. The user can choose from sites and waste streams that are specifically associated with the WIPP WTWBID or a designated user site as long as the input file structure and name are in the correct format (Appendix A and Table 1). To choose a site or input file that is not associated with the WIPP WTWBID, choose the 'OTHER SITE' category when prompted for a site number and PreORG will prompt you for needed information.

PreORG generates one ORIGEN2 input file for a given year (e.g., 1993) for each site and each waste type and names the input file as indicated in the following table (Note: The user can specify a base year as needed for the calculation.). The first four letters of the ORIGEN2 input file indicate the site name, the next two letters describe the type of waste, and the numbers indicate the base year to which all initial inventories are decayed out prior to future decay calculations (Table 1).

Table 1. ORIGEN2 Input Files Created by PreORG

\begin{tabular}{|ccc|}
\hline & PREORG OUTPUT & EXAMPLE FOR ORNL \\
FIYPE OF WASTE & SITE \\
& & \\
\hline \hline CH TRU & XXXXCH93.U5 & ORNLCH93.U5 \\
RH TRU & XXXXRH93.U5 & ORNLRH93.U5 \\
BURIED TRU & XXXXBU93.U5 & ORNLBU93.U5 \\
& & \\
\hline
\end{tabular}

In the WIPP WTWBID data files, activities may be listed that are not broken down into specific isotopes but are grouped together and given the following names: UENR, UDPL, UNAT, UNKN, TBGMAP, and TBGMFP. When PreORG encounters these names, it automatically breaks the activities into the following isotopic breakdowns by multiplying the supplied activity by the activity factor as follows: 


\section{UENR - Enriched Uranium}

The user enters the atom fractions for U234, U235, and U238 at the beginning of the PreORG run for a given site. The enriched uranium atom fractions can be modified for each site if desired.

UDPL - Depleted Uranium

\begin{tabular}{|c|c|}
\hline ISOTOPE & $\begin{array}{c}\text { ACTIVITY } \\
\text { FACTOR }\end{array}$ \\
\hline U238 & 1.00 \\
\hline
\end{tabular}

UNAT - Natural Uranium (General Electric Nuclear Energv, 1989)

\begin{tabular}{|c|c|}
\hline ISOTOPE & $\begin{array}{c}\text { ACTIVITY } \\
\text { FACTOR }\end{array}$ \\
\hline & \\
U234 & 0.000055 \\
U235 & 0.00720 \\
U238 & 0.992745 \\
\hline
\end{tabular}

\section{UNKN - Unknown}

Ignores these activities for lack of a better breakdown

\section{IBGMAP - Mixed Activation Products (see Appendix E)}

\begin{tabular}{|c||c|}
\hline ISOTOPE & $\begin{array}{c}\text { ACTIVITY } \\
\text { FACTOR }\end{array}$ \\
\hline Ba137m & $9.7000 \mathrm{E}-03$ \\
C14 & $1.0000 \mathrm{E}-02$ \\
Co58 & $1.0000 \mathrm{E}-02$ \\
Co60 & $6.7000 \mathrm{E}-01$ \\
Cs137 & $1.0300 \mathrm{E}-02$ \\
Eu152 & $1.0000 \mathrm{E}-02$ \\
Eu154 & $8.5000 \mathrm{E}-02$ \\
Eu155 & $8.5000 \mathrm{E}-02$ \\
Fe59 & $4.0000 \mathrm{E}-02$ \\
Mn54 & $7.0000 \mathrm{E}-02$ \\
\hline
\end{tabular}




\section{TBGMFP - Mixed Fission Products (see Appendix E)}

\begin{tabular}{|c|c|}
\hline IsOTOPE & ACTIVIY \\
FACTOR \\
\hline Ag110 & \\
Ag110m & $5.0200 \mathrm{E}-06$ \\
Ba137m & $2.5070 \mathrm{E}-06$ \\
Cd113m & $2.0090 \mathrm{E}-02$ \\
Ce144 & $5.0000 \mathrm{E}-10$ \\
Cs136 & $2.7840 \mathrm{E}-01$ \\
Cs135 & $5.5570 \mathrm{E}-04$ \\
Cs137 & $5.7500 \mathrm{E}-06$ \\
Eu152 & $2.0090 \mathrm{E}-02$ \\
Eu154 & $3.0000 \mathrm{E}-07$ \\
Eu155 & $3.0380 \mathrm{E}-05$ \\
Gd162 & $7.3530 \mathrm{E}-04$ \\
Nb95 & $9.8620 \mathrm{E}-06$ \\
Nb95m & $1.0530 \mathrm{E}-01$ \\
Pd107 & $1.0590 \mathrm{E}-03$ \\
Pm147 & $8.5000 \mathrm{E}-09$ \\
Pr144 & $7.0220 \mathrm{E}-02$ \\
Rh106 & $2.7840 \mathrm{E}-01$ \\
Ru106 & $4.0510 \mathrm{E}-02$ \\
Sb125 & $4.0510 \mathrm{E}-02$ \\
Sb126 & $7.9850 \mathrm{E}-04$ \\
Sb126m & $1.0940 \mathrm{E}-07$ \\
Se79 & $1.1050 \mathrm{E}-07$ \\
Sm151 & $4.9900 \mathrm{E}-08$ \\
Sn119m & $1.9380 \mathrm{E}-04$ \\
Sn121m & $4.3720 \mathrm{E}-05$ \\
Sn123m & $3.9430 \mathrm{E}-06$ \\
Sn126 & $1.8470 \mathrm{E}-05$ \\
Sr90 & $1.1050 \mathrm{E}-07$ \\
Tb162 & $1.8810 \mathrm{E}-02$ \\
Tc99 & $9.8620 \mathrm{E}-06$ \\
Te125m & $2.8670 \mathrm{E}-06$ \\
Te127 & $2.1760 \mathrm{E}-04$ \\
Te127m & $1.3540 \mathrm{E}-03$ \\
Y90 & $1.3690 \mathrm{E}-03$ \\
Zr93 & $1.8810 \mathrm{E}-02$ \\
Zr95 & $6.4550 \mathrm{E}-07$ \\
& $4.9880 \mathrm{E}-02$ \\
\hline
\end{tabular}




\subsection{Running PreORG}

To run the ORIGEN2 pre-processor on a personal computer (PC), the source code must be obtained from either Jennifer Liscum-Powell, Nuclear Facilities Safety and Risk Analysis, Org. 9364, or Lawrence C. Sanchez, WIPP Performance Assessment Computational Support, Org. 6848. If neither of the two personnel listed is available, the user can contact the WIPP Performance Assessment Computational Support Department, Org. 6848. The user installs PREORG.FOR into a specified directory and compiles PREORG.FOR with a FORTRAN77 compiler. Once an executable file has been created, the user types PREORG at the prompt and presses the carriage return. The user then follows the instructions as they appear on the screen. (Note: All WIPP WTWBID input files must be in the same directory, otherwise PreORG will fail to execute.)

A user directly related to the WIPP project should obtain an OpenVMS account on the WIPP PA Cluster and run the PreORG from the Code Management System (CMS). PreORG resides in the OR library of CMS. The user can locate the executable file-PREORG_PA96.EXE—in the WP\$PRODROOT:[OR.EXE] library. PreORG should be run using command files; examples of these command files are located in the WP\$TESTROOT:[OR2.TEST] directory. 
Intentionally Left Blank 


\subsection{RUNNING ORIGEN2}

To run ORIGEN2 on a PC, the user must follow a few instructions. First, a user ensures that a licensed copy of ORIGEN2 from the Radiation Shielding Information Center (RSIC) of Oak Ridge National Laboratory is installed on the computer. Once ORIGEN2 is installed on a PC, the following directories should be located on the hard drive: C:IORIGEN2, C:IORIGEN2ICODE, C:IORIGEN2LLIBS, C:IORIGEN2LSAMPLES, and C:IORIGEN2UNPUTS. To run the code, the file ORIGEN2.BAT must be present in the C:IORIGEN2 directory and F7713.EER must be located in the C:IORIGEN2ICODE directory along with the executable ORIGEN2.EXE. A listing of the ORIGEN2.BAT file for the PC is given in Appendix B. This file is not issued by RSIC with the ORIGEN2 package. To obtain a copy of the source code, contact either Jennifer Liscum-Powell, Org. 9364, or Lawrence C. Sanchez, Org. 6848, or contact the WIPP PA Computational Support Department, Org. 6848. To run ORIGEN2 without this .BAT file, refer to the ORIGEN2 manual (Croff, 1980).

To run ORIGEN2, simply type the following line at the prompt in the C:IORIGEN2 directory:

\section{C:IORIGEN2 ORIGEN2 filename [return]}

For example, if the ORIGEN2 input file is ORNLCH93.U5, the user would type ORIGEN2 ORNLCH93 to run ORIGEN2. (Note: Do not include the .U5 extension when ORIGEN2 runs, as it will not execute and will write over the input file!) Running ORIGEN2 generates a number of output files; however, the only significant file is the one with the .U6 extension. This file contains the existing desired output of ORIGEN2. It will be a sizable file because ORIGEN2 writes out a vast amount of data to its output files, a large portion of which is not needed by the user.

A user directly related to the WIPP project should obtain an OpenVMS account on the WIPP PA Cluster and run ORIGEN2 from the Code Management System (CMS). The user can locate the executable file-ORIGEN2_PA96.EXE-in the 
WP\$PRODROOT:[OR.EXE] library. ORIGEN2 should be run using command files; examples of these command files are located in WP\$TESTROOT:[or2.test] directory.

PostORG, described in more detail in the next section, was created to process the output files and remove all excessive and irrelevant data for the purposes of the WIPP WTWBID nuclide decay and accumulation calculations. PostORG also creates a master list of nuclides, sums the radionuclide inventories at the base year and creates an ORIGEN2 input file to decay the accumulated base year radionuclide inventories into the future. 


\subsection{DESCRIPTION OF POSTORG}

PostORG was developed to process ORIGEN2 output files with the extension .U6 to remove excessive data and extract the nuclide decay and accumulation data that are of interest for the scope of these calculations. This post-processor uses the following steps:

STEP 1 Prompts user for name of ORIGEN2 output file to be processed (e.g., ORNLCH93.U6 or ORNLC93F.U6).

STEP 2 Prompts user if he/she wishes to generate a ORIGEN2 input file to decay base year (e.g., 1993) data (located in ORNLCH93.SUM file) to a specified time in the future.

STEP 2a If answer for step 2 is yes, ORIGEN2 input file is created with a name like ORNLC93F.U5 (Must run ORIGEN2 with this input file to generate data and then use PostORG to reduce data to ORNLC93F.SUM file).

STEP $2 b$ If answer to step 2 is no, no new ORIGEN2 input file is created.

STEP 3 Reads through output file and separates data by year into individual files (e.g., ORNLCH70.DAT, ORNLCH71.DAT, etc.) and creates a list of these files in an .IND file (e.g., ORNLCH93.IND).

STEP 4 Calls subroutine to add all activities at same years together. (Note: The process only considers isotopes of interest to the WIPP that are identical to those listed in Appendix E of the WIPP Baseline Inventory Report, Rev. 1 [WTAC, 1995a]).

STEP 5 Writes sums to file .SUM (e.g., ORNLCH93.SUM).

STEP 6 Prompts user to indicate whether or not he/she wishes to process another output file

- If yes, return to STEP 1.

- If no, terminate execution.

The resulting output files can be easily imported into a spreadsheet and manipulated as desired by the user. A program listing is provided in Appendix $\mathrm{F}$ and a sample output file is listed in Appendix $G$ along with a listing of the output written to the server where POSTORG is executed. 


\subsection{Running PostORG}

To run the ORIGEN2 post-processor on a PC, the user copies PostORG.for into a specified directory and compiles data with a FORTRAN77 compiler. The source code, POSTORG.FOR, may be obtained by contacting Jennifer Liscum-Powell, Org. 9364, or Lawrence C. Sanchez, Org. 6848, or the WIPP PA Computational Support Department, Org. 6848. Once an executable file has been created, the user types PostORG at the prompt and presses the carriage return. The user then follows the instructions as they appear on the screen. (Note: All ORIGEN2 output files that are to be processed must be in the same directory, otherwise PostORG will fail to execute.)

A user directly related to the WIPP project should obtain an OpenVMS account on the WIPP PA Cluster and run PostORG from the Code Management System (CMS). PostORG resides in the OR library of CMS. The user can locate the executable file POSTORG_PA86.EXE—in the WP\$PRODROOT:[OR.EXE] library. PostORG should be run using command files; examples of these command files are located in the WP\$TESTROOT:[OR2.test] directory. 


\subsection{USING PREORG, ORIGEN2, AND POSTORG}

This section offers step-by-step instructions for running the three codes discussed in this report. By following these instructions, the user should be able to successfully obtain the desired information. There is also a list of warnings for the user to note, particularly when running ORIGEN2 and analyzing the data.

\subsection{Running PreORG, ORIGEN2, and PostORG on a PC}

To successfully run the three codes discussed in this report, a user should follow the instructions listed below:

\section{PreORG}

STEP 1 Change directory to the one containing PREORG.EXE.

STEP 2 At prompt, type PREORG and hit return.

STEP 3 Answer questions as they appear on screen.

\section{ORIGEN2}

STEP 1 Copy all input files with .U5 extension into ORIGEN2 directory.

STEP 2 For each ORIGEN2 input file specified to run, type ORIGEN2 filename without extension at the prompt and hit return.

STEP 3 Copy all ORIGEN2 output files with .U6 extension to directory containing POSTORG.EXE.

STEP 4 Delete all unnecessary output files with extensions other than .u6.

\section{PostorG}

STEP 1 Change directory to one containing POSTORG.EXE.

STEP 2 At prompt, type POSTORG and hit return.

STEP 3 Answer questions as they appear on screen.

(Note: POSTORG output files may be imported into EXCEL spreadsheet to view and print [optional]). 


\subsection{Running PREORG, ORIGEN2, and POSTORG on a VMC Computer Platform}

To successfully run the three codes discussed in this report on VMS, a user should develop a command file to run the codes sequentially: (1) PREORG, to generate ORIGEN2 input files, (2) ORIGEN2, to generate radionuclide decay and accumulation data, and (3) POSTORG, to extract useful information from ORIGEN2 output files.

A sample command file is shown in Figure 6.2.1 that runs ORIGEN2 for CH-TRU waste from the Hanford and Oak Ridge waste generation sites.

\subsection{Cautions to Observe when Running PreORG, ORIGEN2, and PostORG}

To successfully run the three codes discussed in this report, please observe the following cautions listed below:

\section{PreORG Cautions}

- All input files for a given site and given waste type must have the correct name (see Appendix A).

- All input files must be in the correct format (see Appendix A).

\section{ORIGEN2 Cautions}

- Input file must be in correct directory.

- When running the code, do not include. U5 extension on input or ORIGEN2 will fail to execute and write over input file.

- Disable EMM386.EXE memory extender.

- ORIGEN2 does not warn you if errors are present in input. The user is responsible for checking output to make sure data makes sense.

- ORIGEN2 has problems (loses accuracy and yields isotope concentrations that are not feasible) when instructed to decay each initial year of inventory out and then print the years all together in one output table. Thus, PreORG writes commands to decay out each year to the given base year (e.g., 1993) individually and outputs each year's in its wn output table (see Appendix C). 


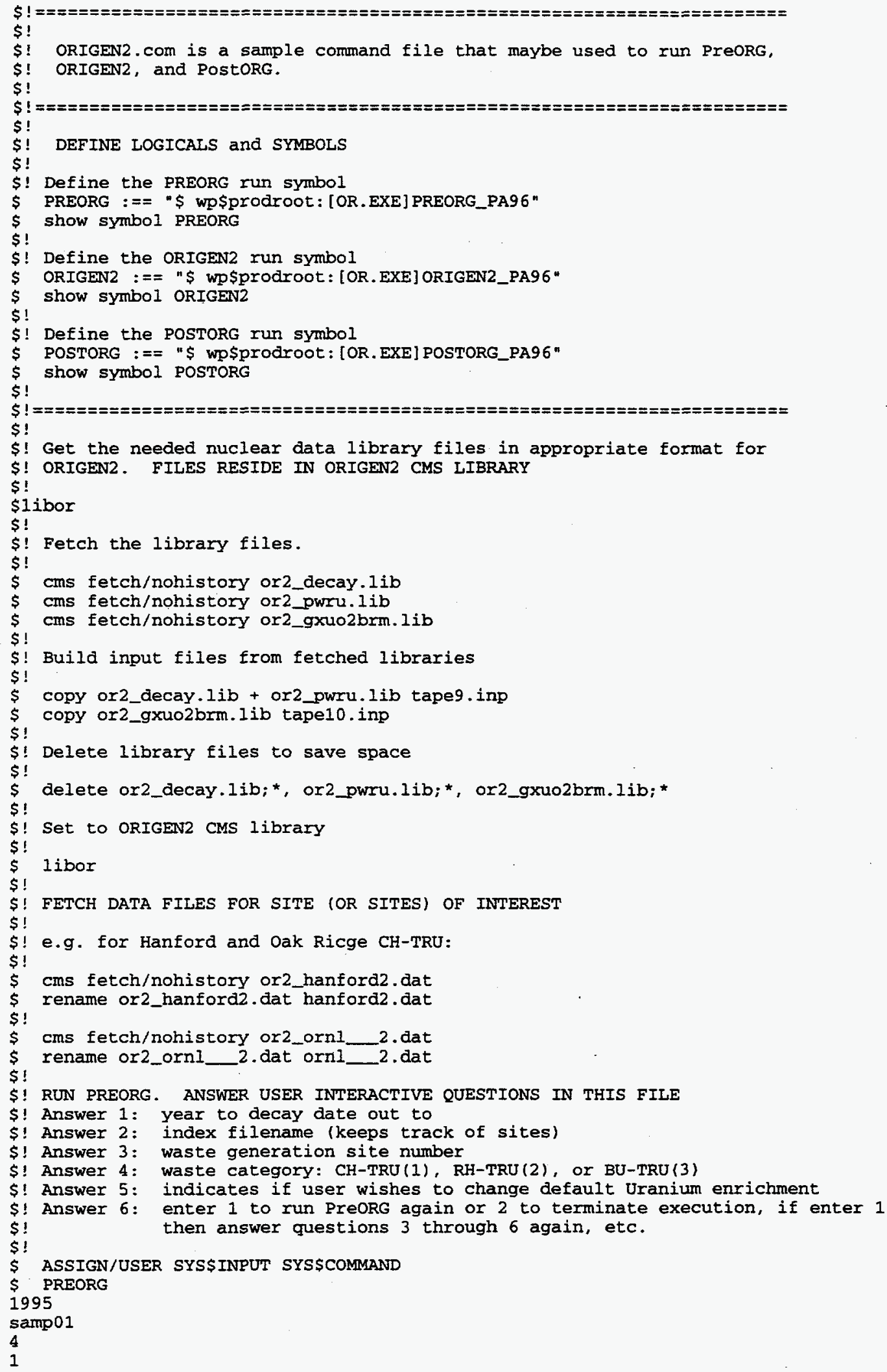

Figure 6.2.1 Sample command file used to run PreORG, ORIGEN2, and PostORG. 


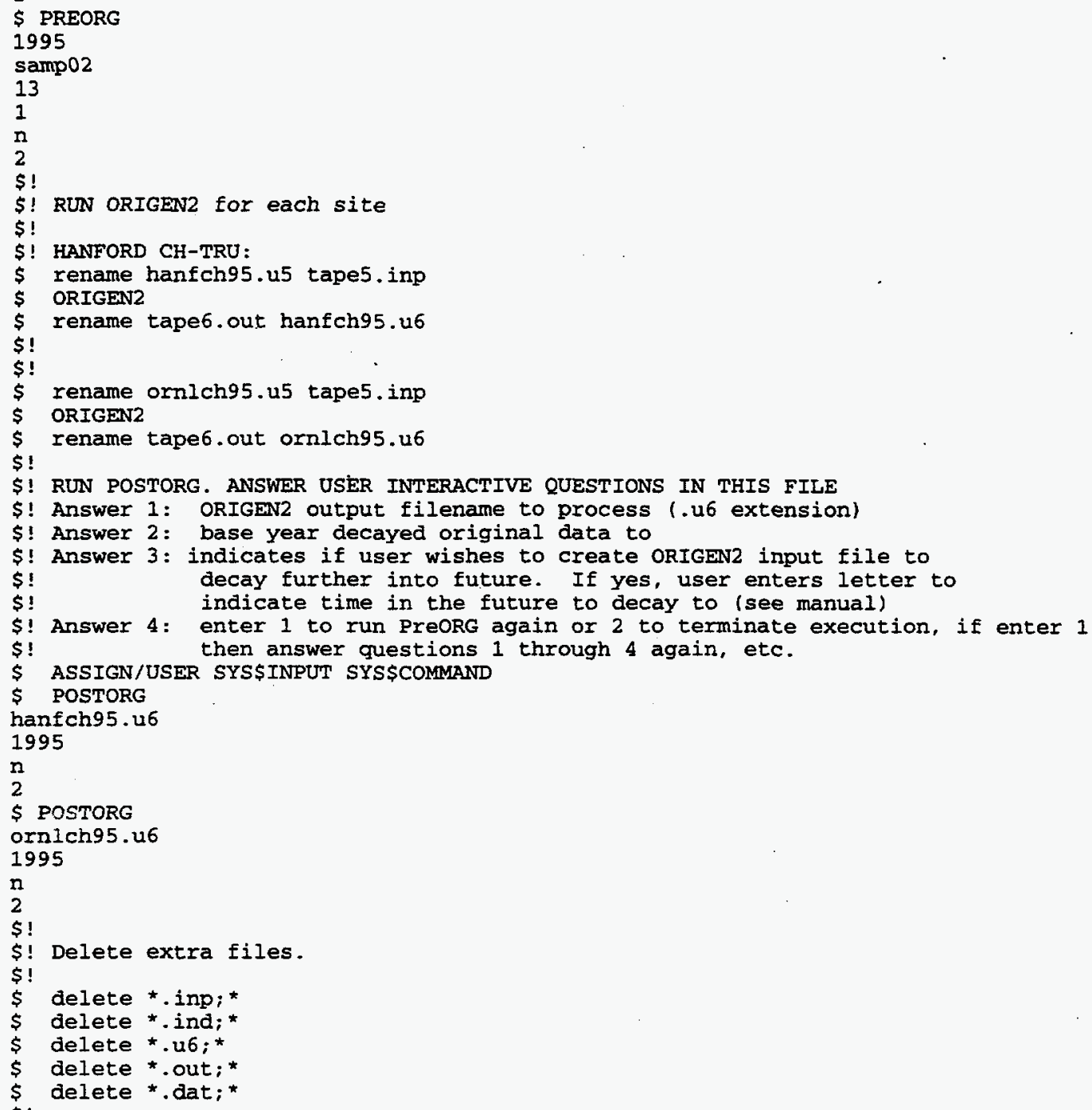

Figure 6.2.1 Sample command file used to run PreORG, ORIGEN2, and PostORG (continued). 
- ORIGEN2 includes an "ADD" command that will supposedly add nuclide concentrations stored in one output vector to another one nuclide at a time. However, when this command is used to add final nuclide data together after all initial inventories were decayed out to the base year, inaccuracies are observed along with activities for isotopes that are physically impossible. This problem with ORIGEN2 is avoided by first decaying all initial inventories to a common year by running ORIGEN 2 with the input files created by PreORG and then running PostORG to process the output and generate an input file for ORIGEN2 to decay isotopes into the future (Appendix G).

- ORIGEN2 is limited in how many output vectors it will print in its output file. For a given initial nuclide concentrations, ORIGEN 2 can output 11 vectors without error. Thus, the initial values can be included in the output along with 10 vectors containing data at 10 different times.

- When using the decay command several times to look at nuclide concentrations at various time intervals in the future, please note that the time interval or step of the decay is determined by subtracting the time given in the previous decay command from the time given in the present one. Please make sure the decay time is greater in the latter command or errors will result (Appendix C).

\section{Postorg Cautions}

- Input file names must be 11 characters long and include the extension .u6.

- Input files must be in the same directory as POSTORG.EXE.

- Must enter the same base year as used in PREORG (e.g., 1993). 
Intentionally Left Blank 


\subsection{SUMMARY}

PreORG and PostORG were created to automate decay calculations using ORIGEN2 for the WIPP WTWBID. The pre-processor, PreORG, translates WTWBID data files into ORIGEN2 input files by requiring interaction with the user. The user indicates which waste-generation site and waste type is of interest and the base year to which he/she wishes to decay all years of inventory. A user can choose to use a site other than the 20 currently contributing to the WIPP inventory on a site-level basis; however, the user should be cautioned that the naming convention is the same and PreORG will fail to execute if the data file does not exist in the correct format. PostORG processes the ORIGEN2 output files, eliminating excess data and thus creating a more manageable output. PostORG can also generate an ORIGEN2 input file to decay the base year data for a given waste generation site up to 10,000 years into the future. These two codes enable the automation of ORIGEN2 decay and accumulation calculations for the WIPP WTWBID. 
Intentionally Left Blank 


\subsection{FURTHER READING}

Further generalized discussion on ORIGEN2 can be found in Croff, 1983.

Detailed information on the validation (as applied to analysis of PWR spent fuel) and functional requirements (related to long-term storage of LWR spent fuel) of ORIGEN2 can be found in Hermann et al., 1995, and Broadhead et al., 1995. 
Intentionally Left Blank 


\subsection{REFERENCES}

Broadhead, B.L., M.D. DeHart, J.C. Ryman, J.S. Tang, and C.V. Parkss. 1995. Investigation of Nuclide Importance to Functional Requirements Related to Transport and Long-Term Storage of LWR Spent Fuel. ORNL/TM-12742. Oak Ridge, TN: Oak Ridge National Laboratory.

Croff, A.G. 1980. A User's Manual for ORIGEN2 Computer Code. ORNL/TM-7175. Oak Ridge, TN: Oak Ridge National Laboratory.

Croff, A.G. 1983. "ORIGEN2: A Versatile Computer Code for Calculating the Nuclide Compositions and Characteristics of Nuclear Materials," Nuclear Technology. Vol. 62, no. 3, 335-352.

Hermann, O.W., S.M. Bowman, C.V. Parks, and M.C. Brady. 1995. Validation of the Scale System for PWR Spent Fuel Isotopic Composition Analyses. ORNL/TM-12667. Oak Ridge, TN: Oak Ridge National Laboratory.

Kaplan, I. 1964. Nuclear Physics. 2nd ed. Reading, MA: Addison-Wesley Publishing Company. pp. 229, 242-243.

Liscum-Powell, J., R. Carter, and H. Trellue. 1996. "WIPP PA Analysis Report for ORIGEN2 Suite," WBS\# 1.1.6.1, SWCF-A:1.4.01.6:SFT:VH511196:QA:ORIGEN2: Analysis Package for Initial Radionuclide Inventory, April 22, 1996. (Copy on file in the Sandia WIPP Central Files, Sandia National Laboratories, Albuquerque, NM as WPO 36974.)

Tuli, J.K. 1985. Nuclear Wallet Cards. Upton, NY: National Nuclear Data Center, Brookhaven National Laboratory. (Copy on file in the Sandia WIPP Central Files, Sandia National Laboratories, Albuquerque, NM as WPO 41010.)

Turner, J.E. 1986. Atoms, Radiation, and Radiation Protection. New York, NY: Pergamon Press. pp. 39, 64. 
Walker, F.W., J.R. Parrington, and F. Feiner. 1989. Nuclides and Isotopes: Chart of the Nuclides. 14th ed. San Jose, CA: General Electric Company.

WTAC (WIPP Technical Assistance Contractor). 1995a. Waste Isolation Pilot Plant Transuranic Waste Baseline Inventory Report. CAO-94-1005, Revision 1. [Carlsbad, NM]: WIPP Technical Assistance Contractor for U.S. Department of Energy. Vols. 1-3.

WTAC (WIPP Technical Assistance Contractor). 1995b. Transuranic Waste Baseline Inventory Report (Revision 2). DOE/CAO-95-1121. Carlsbad, NM: Carlsbad Area Office Technical Assistance Contractor. 
APPENDIX A: SAMPLE PREORG.FOR DATA INPUT FILE 
Intentionally Left Blank

A-2 


\section{Appendix A: Sample PREORG.FOR Data Input File}

On the following pages is a sample input file for "PREORG.FOR" containing data from the Waste Isolation Pilot Plant (WIPP) Baseline Inventory Database (BID) for the Oak Ridge National Laboratory (ORNL) site for contact-handled transuranic (CH TRU) waste from 1970 to 1977. (Note: This is a portion of the data file for ORNL CH TRU waste).

All input files used with the pre-processor must follow the format presented in this appendix. All input files must also have the proper name structure as indicated in Table A-1 below. The input filename must be eight characters long, excluding the extension-DAT - that is required. The eighth position of the input file name must indicate the type of waste contained in the file: a $\mathbf{2}$ for CH TRU waste, a $\mathbf{3}$ for remotely handled (RH) TRU waste, or a $\mathbf{7}$ for Buried TRU waste.

Table A-1. PreORG.for Input Filename Structure

\begin{tabular}{|ccc|}
\hline TYPE OF WASTE & PREORG.FOR INPUT FILENAME & EXAMPLE FOR ORNL STTE \\
\hline \hline CH TRU & $x \times x \times x \times x 2 . D A T$ & ORNL_2.DAT \\
RH TRU & $x \times x \times x \times 3$ 3.DAT & ORNL_3.DAT \\
BURIED TRU & $x \times x \times x \times 7$.DAT & ORNL_7.DAT \\
& & \\
\hline
\end{tabular}

To run the ORIGEN2 pre-processor to generate an ORIGEN2 input file, all input files must be identical in format to the file presented in this appendix. For the purposes of this report, the first two lines are added to indicate the columns in which data are read. (Note: In order for PREORG.FOR to properly read an input file, all data must be located in the designated column because it uses character strings to read and manipulate the data.) If the year is not given in columns 1 to 4, PREORG.FOR will not work. Likewise, it is necessary that the isotope names start in column 3 and that the initial activities are listed in Curies in columns 41 through 50. The end of the data for each year is indicated by the presence of ' $Z Z Z Z Z$ ' in columns 3 through 7 . The data shown in columns 11 to 16 are not necessary for the preprocessor to run properly, but if 
the data are deleted, they must be replaced with spaces to maintain the column integrity in order for the initial activities to be read in correctly.

Following the sample file, Table A-2 lists specific input files for the WIPP Transuranic Waste BID (TWBID). 
$\begin{array}{lccccr}1 & 2 & 3 & 4 & 5 & 5\end{array}$

1234567890123456789012345678901234567890123456789012345

ORNL STORED CONTACT HANDLED TRU WASTE

$1970 \longleftarrow$ Year of Nuclide Inventories

PU239 $20 \lcm{31}$

AM2 $41 \quad 20 \quad 36$

$\begin{array}{lll}\mathrm{U} 233 & 20 \quad 23\end{array}$

AM2 $43 \quad 20 \quad 37$

CF252 $20 \quad 44$

CM244 $20 \quad 45$

U235 $20 \quad 25$

UNKN 00

ZZZZZ $99 \quad 0$

11

12

13

14

1971

PU238 $20 \quad 30$

PU239 $20 \quad 31$

PU2 $40 \quad 20 \quad 32$

PU241 $20 \quad 33$

AM241 $20 \quad 36$

$\begin{array}{lll}\text { U233 } & 20 & 23\end{array}$

$\begin{array}{lll}\text { CO60 } & 10 & 07\end{array}$

AM243 $20 \quad 37$

BK249 $20 \quad 46$

CF252 $20 \quad 44$

CM242 $20 \quad 38$

CM244 $20 \quad 45$

NP237 $20 \quad 28$

U235 $20 \quad 25$

UNKN . 00

ZZZZZ $99 \quad 0$

1972

PU238 $20 \quad 30$

PU239 $20 \quad 31$

PU240 $20 \quad 32$

PU241 $20 \quad 33$

AM2 $41 \quad 20 \quad 36$

AM243 $20 \quad 37$

BK249 $20 \quad 46$

CF252 $20 \quad 44$

CM244 $20 \quad 45$

NP237 20 28

TH232 $20 \quad 18$

$\begin{array}{lll}\mathrm{U} 235 & 20 & 25\end{array}$

$\begin{array}{lll}\text { U238 } & 20 & 27\end{array}$

UNKN 00

ZZZZZ

$$
1973
$$

PU238 990

PU239

$20 \quad 30$

PU240 $20 \quad 32$

PU241 $20 \quad 33$

AM241 $20 \quad 36$

$\begin{array}{lll}\mathrm{U} 233 & 20 & 23\end{array}$

AM2 $43 \quad 20 \quad 37$

BK2 $49 \quad 20 \quad 46$

CF252 $20 \quad 44$

CM2 $44 \quad 20 \quad 45$

NP237 $20 \quad 28$

$\begin{array}{lll}\text { U235 } 20 & 25\end{array}$

ZZZZZ $99 \quad 0$ 1974

군

7. $0000 \mathrm{E}+00$

2. $8400 \mathrm{E}-04$

2. $8400 \mathrm{E}-04$

1. $0100 \mathrm{E}-04$

1. $0000 \mathrm{E}-06$

4. $4300 \mathrm{E}+00$

7. $5000 \mathrm{E}-05$

3. $6000 \mathrm{E}+01$

$4.2600 \mathrm{E}+03$

3. $2700 E+01$

1. $7600 \mathrm{E}+00$

4. $0800 \mathrm{E}+02$

8. $8000 \mathrm{E}+02$

1. $5200 \mathrm{E}-01$

1. $0000 \mathrm{E}-06$

2. $4800 \mathrm{E}+00$

3. $6200 \mathrm{E}+00$

1. $1400 \mathrm{E}+00$

3. $3200 \mathrm{E}+01$

4. $9300 \mathrm{E}+02$

3. $2300 E+00$

4. $1000 \mathrm{E}-04$

3. $6200 \mathrm{E}+00$

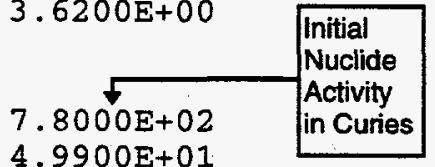

$4.9900 \mathrm{E}+01$

4. $6900 \mathrm{E}-01$

3. $0000 E+03$

1. $6200 \mathrm{E}+02$

3. $7000 \mathrm{E}+00$

2. $0100 \mathrm{E}-03$

5. $5300 \mathrm{E}-01$

4. $2000 \mathrm{E}+02$

1. 0900E-01

3. 3400E-02

1. $6600 \mathrm{E}-04$

2. 2000E-05

1. $0800 \mathrm{E}+02$

5. $8400 \mathrm{E}+01$

3. $6600 \mathrm{E}+00$

3. 5900E-01

8. $4000 \mathrm{E}+01$

1. $3900 \mathrm{E}+01$

2.1900E+00

2. $2000 \mathrm{E}+00$

1. $8200 \mathrm{E}+00$

5. $3500 \mathrm{E}+01$

5. $5100 \mathrm{E}+02$

6.1800E-03

2. 3100E-04

\section{ORIGEN2 TITLE}

Nuclide Name

Indicates end of inventory for current year has been reached. 


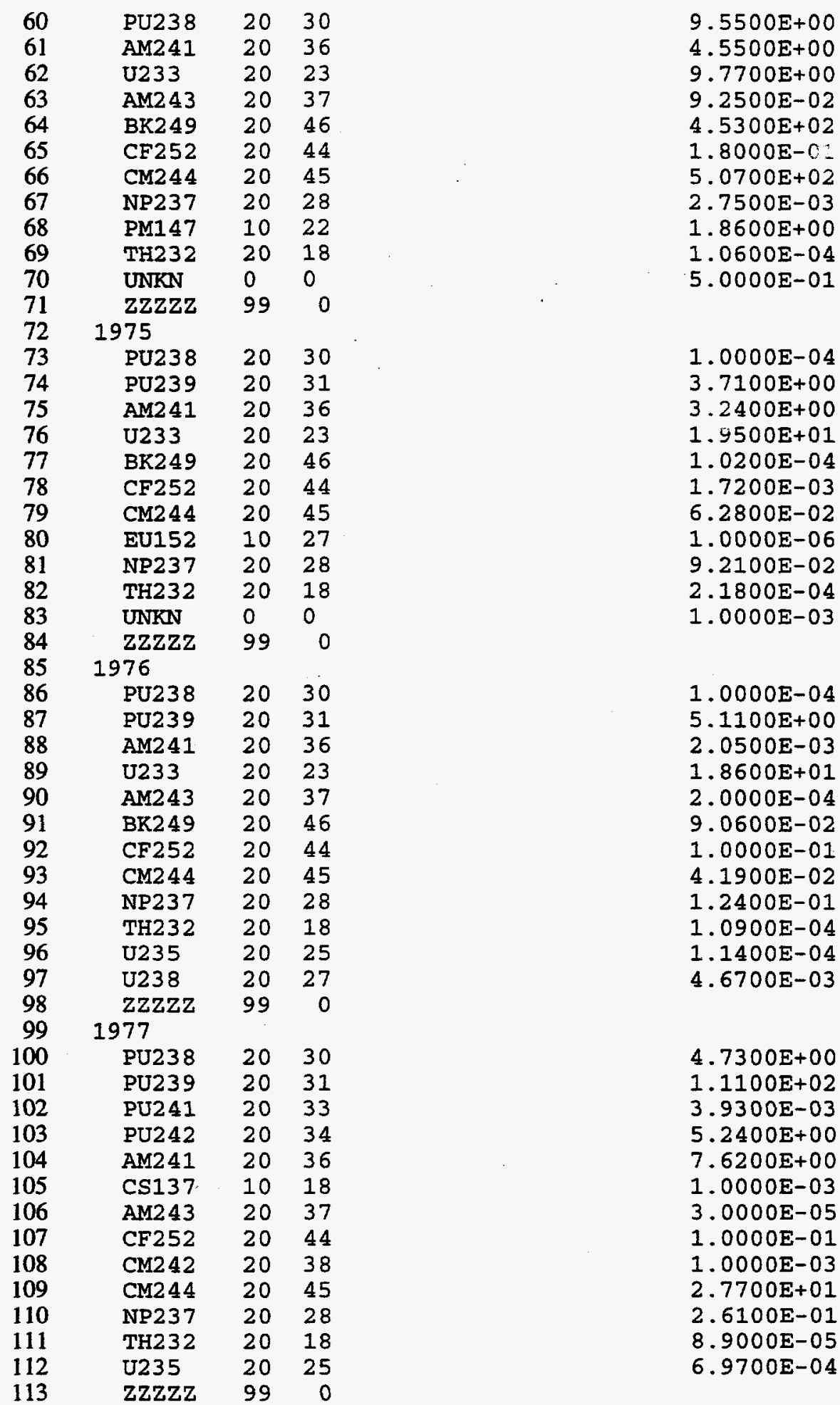


Table A-2. Acceptable WIPP TWBID Data Files for PreORG Input

\begin{tabular}{|c|c|c|c|}
\hline \multirow[b]{2}{*}{ SITE } & \multicolumn{3}{|c|}{ WIPP TWBID DATA FILENAME } \\
\hline & CH-TRU & RH-TRU & BURIED TRU \\
\hline ARCO & arcomp_2.dat & arcomp_3.dat & - \\
\hline Argonne & argonne2.dat & - & - \\
\hline ETEC & etec_sl2, dat & - & - \\
\hline Hanford & hanford2.dat & hanford3.dat & hanford7. dat \\
\hline INEL & inel__.dat & inel__3.dat & \\
\hline Knolls & - & knolls_3.dat & - \\
\hline LB Lab & 1_b_lab2.dat & - & - \\
\hline LANL & $\operatorname{lanl} 2 . \operatorname{dat}$ & $\operatorname{lan} 1 \_3$. dat & 一 \\
\hline LLNL & $\ln 1 \ldots 2$. dat & - & - \\
\hline Mound & mound__ 2.dat & - & - \\
\hline MURR-DOE & murr___ dat & - & - \\
\hline NTS & nts___ 2.dat & nts__ 3. dat & — \\
\hline ORNL & ornl___ dat & ornl__3. dat & ornl__ 7. dat \\
\hline Paducah & paducah2.dat & - & - \\
\hline Pantex & pantex_2.dat & - & - \\
\hline Rocky Flats & rocky_f2.dat & - & - \\
\hline Savannah River & savanna2.dat & savanna3.dat & savanna7.dat \\
\hline SNL/NM & sn1_nm_2.dat & - & snl_nm_7.dat \\
\hline USARMY & armymc_2.dat & armymc_3.dat & - \\
\hline West Valley & w_val_d2.dat & w_val_d3.dat & w_val_d7.dat \\
\hline
\end{tabular}

A typical PreORG run. A listing of what is written to the server follows on the next pages. 

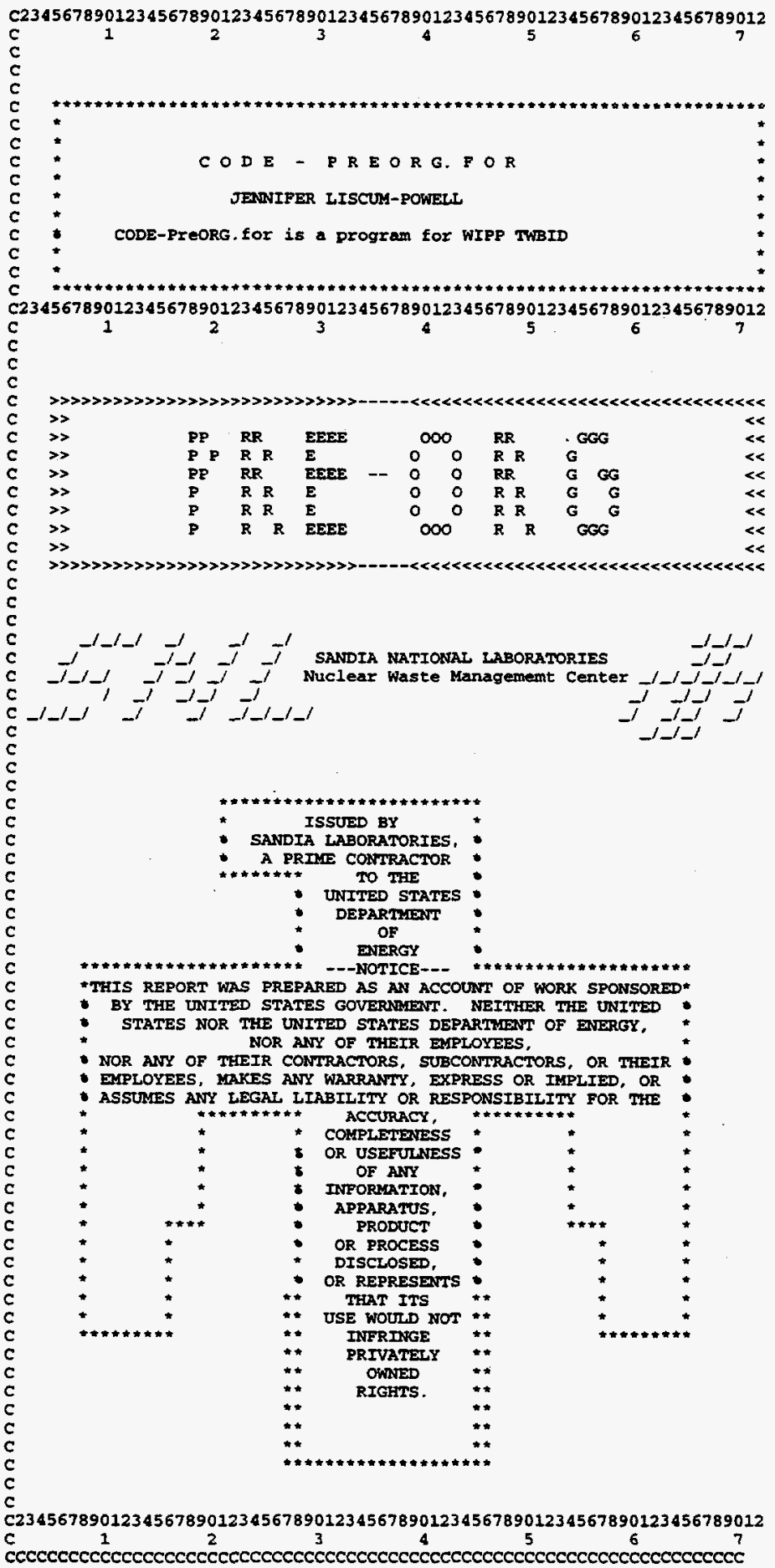

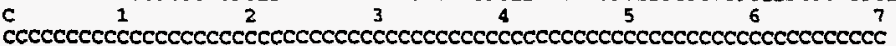

THIS PROGRAM TRANSLATES DATA IN THE WIPP BID INTO ORIGEN2 INPUT FILES TO DECAY INVENTORIES IN THE USER SPECIFIED SITE DATA FILE TO THE SAYE YEAR AS GIVEN BY THE USER.

BYTER YEAR WISH TO DECAY ALCL INVENTORY DATA TO:

ecccecccccccecccececceccecccccccc $c c c c c c c c c c c c c c c c c c c c c c c c c c c c c c c c c c c c c$ 1995 USER ANSWER

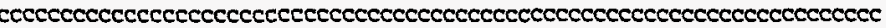

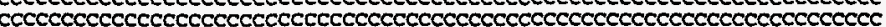

FOR YOUR REFERENCE PREORG.FOR WILL GENERATE A IIST OF THE ORIGEN2 INPUT FILES CREATED DURING THIS RUN

PLEASE ENTER THE NAME OF THE LIST FILE 
6 CHARACTERS OR LESS:

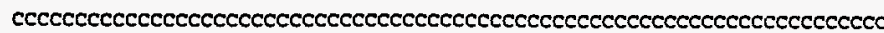

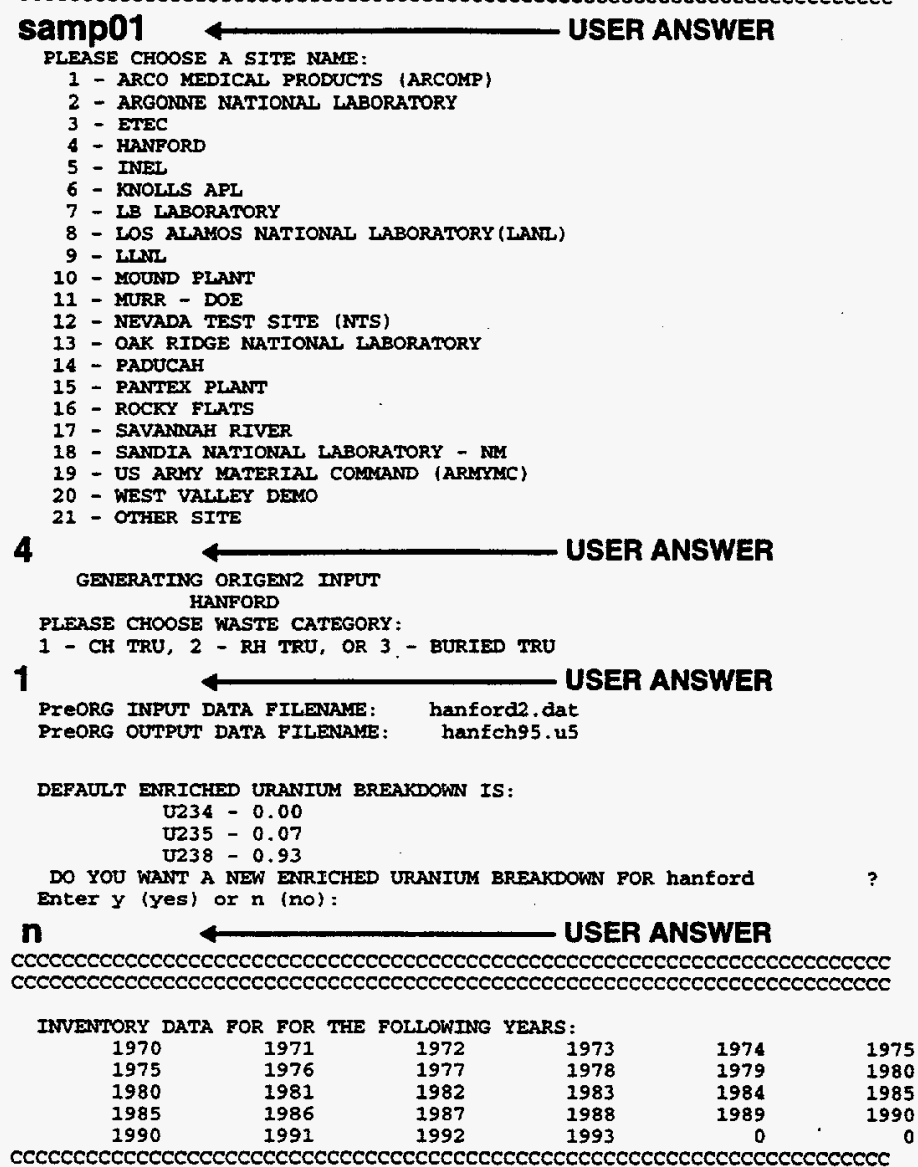

WOULD YOU LIKE TO RUN PROGRAM AGAIN TO GMMERATE

MORE ORIGEN2 INPUT FILES? 1-YES 2-NO

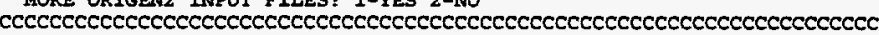

1

4 SITE NAME.

USER ANSWER

PLEASE CHOOSE A SITE NAME:

1 - ARCO MEDICAL PRODUCTS (ARCOMP

2 - ARGONRE NATIONAL LLABORATORY

3 - ETEC

4 - HANEORD

5 - INE

6 - KNOLLS API

7 - LB LABORATORY

- LOS ALAMOS NATIONAL LABORATORY (LANL)

9 - LLNR

10 - MOUND PLANT

11 - MURR - DOE

12 - NEVADA TEST SITE (NTS)

13 - OAK RIDGE NATIONAI LABORATORY

14 - PADUCAF

15 - PANTEX PLANT

16 - ROCKY FLATS

17 - SAVANNAH RIVER

18 - SANDIA NATIONAL LABORATORY - ND

19 - US ARMY MATERIAL COMMAND (ARMYMC)

20 - WEST VALLEY DEMO

21 - OTHER SITE

8

GENERATING ORIGEN2 INPUT

USER ANSWER

PLEASE CHOOS

1 - CH TRU, 2 - RH TRU, OR 3 - BURIED TRU

2

PreORG INPUT DATA FILINAANE: $\quad \operatorname{lanl} 3$. da
PreORG OUTPUT DATA FILENAAME:

DEFAULT ENRICHED URANIUT BREAKDOWN IS

$\mathrm{U} 234-0.00$

$\mathrm{U} 235=0.07$
$\mathrm{U} 238=0.93$

D YOU WANT A NEW ENRICHED URANTUM BREAKDOWN FOR lanI_-

Enter $y$ (yes) of $n$ (no) 


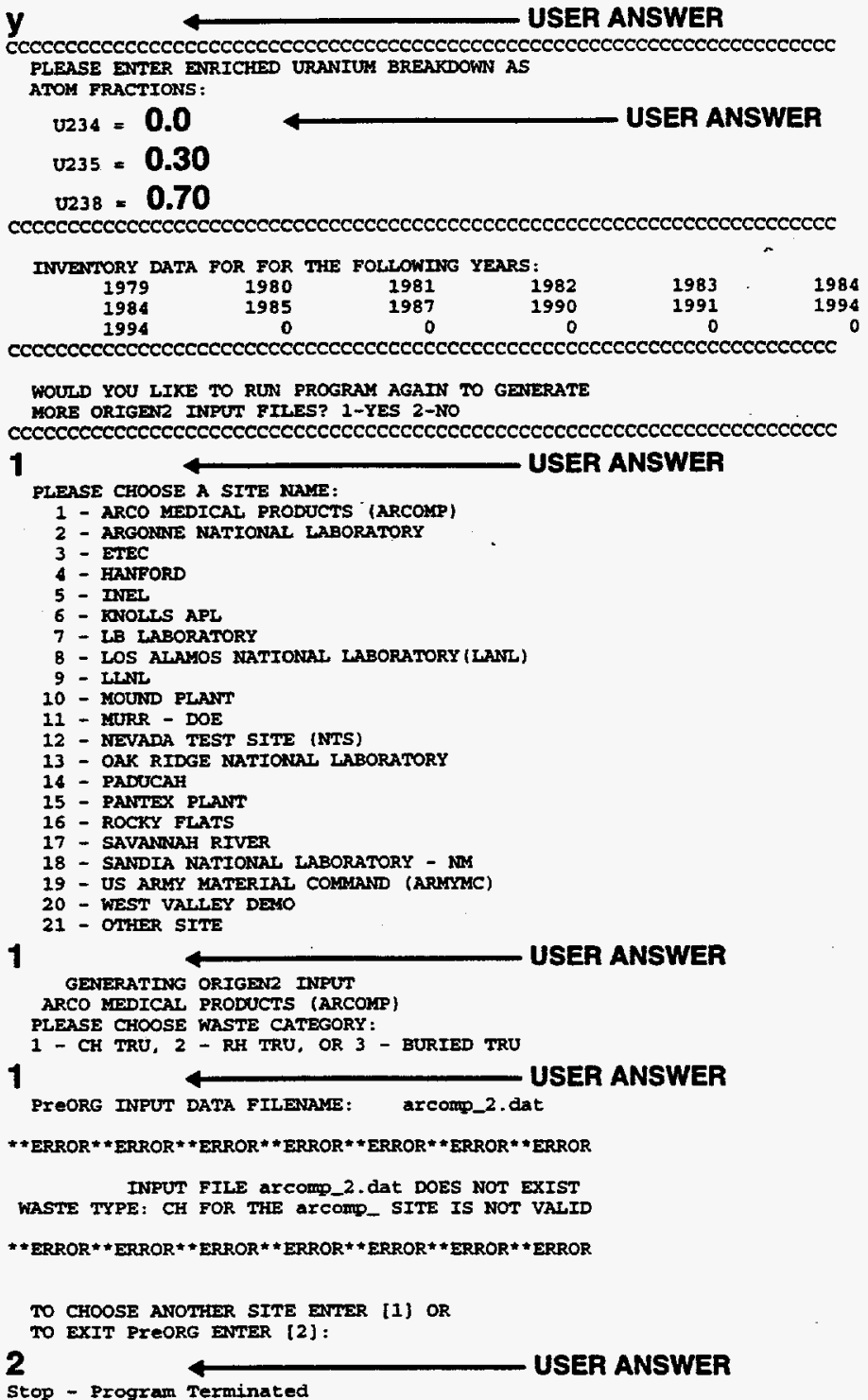


APPENDIX B: ORIGEN2.BAT FILE LISTING FOR A PC 
Intentionally Left Blank 


\section{APPENDIX B: ORIGEN2.BAT FILE LISTING FOR A PC}

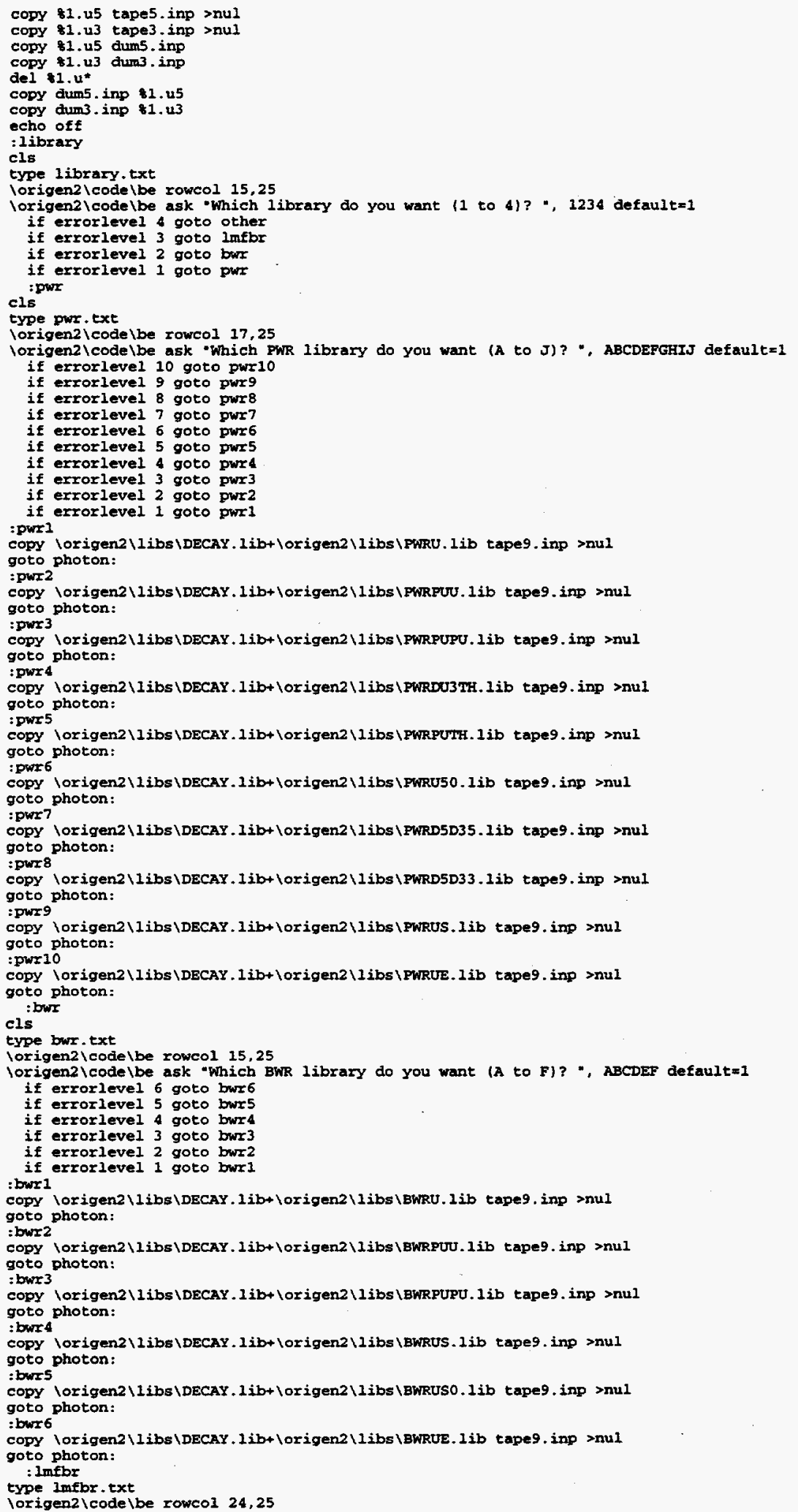




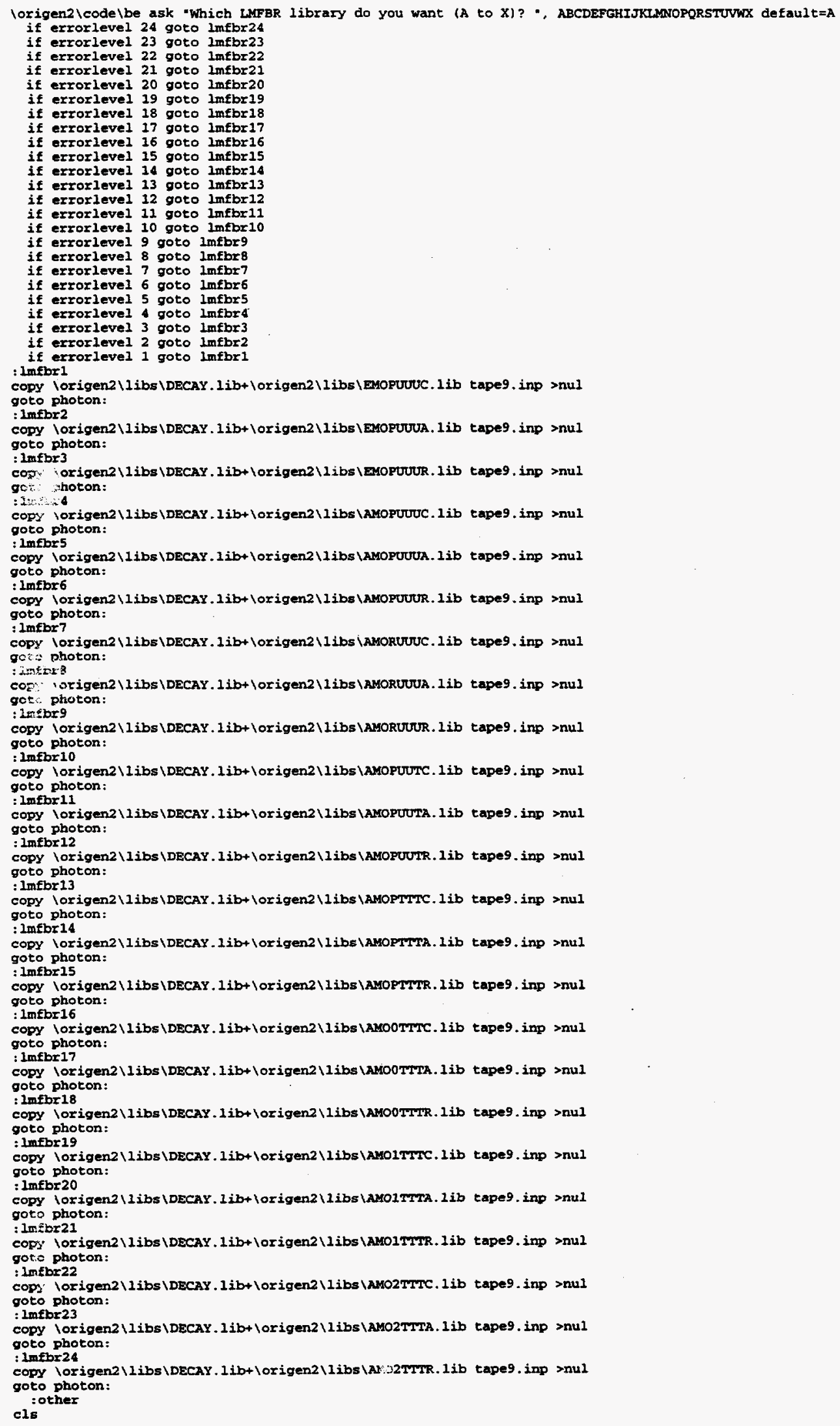




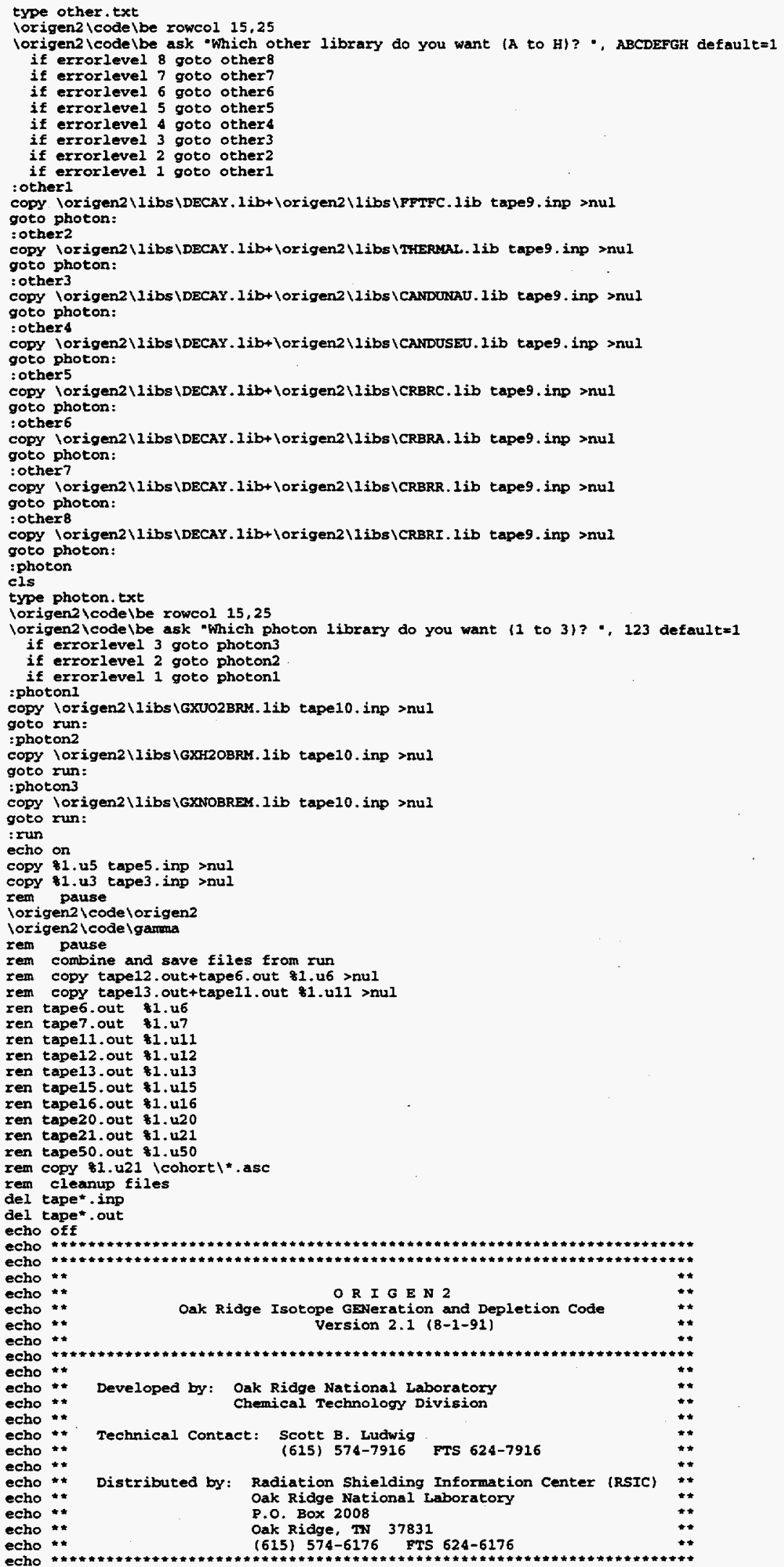




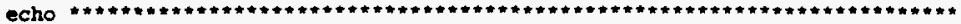

echo ** Execution continuing

echo accong

echo :* Version $2.1(8-1-91)$ for mainframes and 80386 or 00486 PCs

echo **

echo :

年

echo on 
APPENDIX C: ORIGEN2 COMMAND DESCRIPTIONS AND SAMPLE ORIGEN2 INPUT FILE CREATED BY PREORG 
Intentionally Left Blank 


\section{APPENDIX C: ORIGEN2 Command Descriptions and Sample ORIGEN2 Input File Created by Preorg}

\section{C.1 ORIGEN2 Command Description}

For the calculations performed by ORIGEN2 with the input files generated by PreORG, the commands described in Table C-1 are utilized. Table C-1 list the commands in the order in which they appear in the sample ORIGEN2 input file listed in this appendix. The descriptions offered in the table are general and come from the ORIGEN2 manual (Croff, 1980). The table also shows the maximum number of times the command can be used in a given input file.

Table C-1. Description of ORIGEN2 Commands Used in PreORG

\begin{tabular}{|c|c|c|}
\hline $\begin{array}{l}\text { ORIGEN2 } \\
\text { COMMAND }\end{array}$ & $\begin{array}{l}\text { MAXIMUM } \\
\text { NUMBER }\end{array}$ & DESCRIPTION \\
\hline BAS & 10 & $\begin{array}{l}\text { Supplies case basis printed in ORIGEN2 output file } \\
\text { Consists of up to } 71 \text { alphanumeric characters }\end{array}$ \\
\hline TIT & 20 & $\begin{array}{l}\text { Case title } \\
\text { Consists of up to } 71 \text { alphanumeric characters }\end{array}$ \\
\hline $\begin{array}{l}\text { RDA } \\
\text { LIP }\end{array}$ & $\begin{array}{c}300 \\
5\end{array}$ & $\begin{array}{l}\text { Comment card among input commands added by user to explain input file } \\
\text { Controls the printing of the input data libraries }\end{array}$ \\
\hline LIB & 5 & Reads decay and cross-section libraries \\
\hline $\begin{array}{l}\text { PHO } \\
\text { INP }\end{array}$ & $\begin{array}{c}5 \\
15\end{array}$ & $\begin{array}{l}\text { Reads photon production rate per disintegration in } 18 \text { energy groups } \\
\text { Calls for nuclide composition, continuous nuclide feed rate, or continuous } \\
\text { removal rate to be read }\end{array}$ \\
\hline DEC & 150 & Decay nuclide concentration data in one vector for a single decay interval \\
\hline $\begin{array}{l}\text { MOV } \\
\text { HED }\end{array}$ & $\begin{array}{l}99 \\
50\end{array}$ & $\begin{array}{l}\text { Moves nuclide composition in one vector to another vector, nuclide by nuclide } \\
\text { Assians headinas to a specified vector }\end{array}$ \\
\hline OPTA & 20 & $\begin{array}{l}\text { Specifies which output table types (nuclide, element or summary) are to be } \\
\text { printed for the actinides }\end{array}$ \\
\hline OPTF & 20 & $\begin{array}{l}\text { Specifies which output table types (nuclide, element or summary) are to be } \\
\text { printed for the fission products }\end{array}$ \\
\hline OPTL & 20 & $\begin{array}{l}\text { Specifies which output table types (nuclide, element or summary) are to be } \\
\text { printed for the activation products }\end{array}$ \\
\hline $\begin{array}{l}\text { OUT } \\
\text { ADD } \\
\text { STP }\end{array}$ & $\begin{array}{l}20 \\
30 \\
\mathrm{n} / \mathrm{a}\end{array}$ & $\begin{array}{l}\text { Calls for calculated results in some or all output vectors to be printed to a file } \\
\text { Adds the nuclide concentration data of two vectors together, nuclide by nuclide } \\
\text { Executes the set of commands preceding the STP command, then reads and } \\
\text { executes commands that follow. }\end{array}$ \\
\hline END & 1 & Terminates execution of ORIGEN2 \\
\hline
\end{tabular}




\section{C.2 ORIGEN2 Output Tables Description}

As described in Section 2.2, ORIGEN2 can calculate several different characteristics of nuclear materials. The following table lists output tables that can be generated by ORIGEN2. The user can control whether or not any of the first 24 tables will be printed; the remaining are always included in the output file. This table can be found in the ORIGEN2 manual (Croff, 1980). For the purposes of the calculations that are desired from the Waste Isolation Pilot Plant (WIPP) Baseline Inventory Database (BID) input files, only Table 7 (see Table C-2) is printed to the output file.

Table C-2. Description of ORIGEN2 Output Tables

\begin{tabular}{|c|c|c|}
\hline TABLE \# & DESCRIPTION & UNITS \\
\hline $\begin{array}{l}1 \\
2 \\
3 \\
4 \\
5 \\
6 \\
7 \\
8 \\
9 \\
10 \\
11 \\
12 \\
13 \\
14 \\
15 \\
16 \\
17 \\
18 \\
19 \\
20 \\
21 \\
22 \\
23 \\
24 \\
25 \\
26 \\
27 \\
28\end{array}$ & $\begin{array}{l}\text { Isotopic composition of each element } \\
\text { Isotopic composition of each element } \\
\text { Composition } \\
\text { Composition } \\
\text { Composition } \\
\text { Composition } \\
\text { Radioactivity (total) } \\
\text { Radioactivity (total) } \\
\text { Thermal power } \\
\text { Thermal power } \\
\text { Radioactivity (total) } \\
\text { Radioactivity (total) } \\
\text { Radioactive inhalation hazard } \\
\text { Radioactive inhalation hazard } \\
\text { Radioactive ingestion hazard } \\
\text { Radioactive ingestion hazard } \\
\text { Chemical ingestion hazard } \\
\text { Chemical ingestion hazard } \\
\text { Neutron absorption rate } \\
\text { Neutron absorption rate } \\
\text { Neutron-induced fission rate } \\
\text { Neutron-induced fission rate } \\
\text { Radioactivity (alpha) } \\
\text { Radioactivity (alpha) } \\
\text { (alpha,n) neutron production } \\
\text { Spontaneous fission neutron production } \\
\text { Photon emission rate } \\
\text { Set test parameter ERR }\end{array}$ & $\begin{array}{c}\text { atom fraction } \\
\text { weight fraction } \\
\text { gram-atoms } \\
\text { atom fraction } \\
\text { grams } \\
\text { weight fraction } \\
\text { Ci } \\
\text { fractional } \\
\text { watts } \\
\text { fractional } \\
\text { Bq } \\
\text { fractional } \\
\mathrm{m}^{3} \text { air } \\
\text { fractional } \\
\mathrm{m}^{3} \text { water } \\
\text { fractional } \\
\mathrm{m}^{3} \text { water } \\
\text { fractional } \\
\text { neutrons/sec } \\
\text { fractional } \\
\text { fissions/sec } \\
\text { fractional } \\
\text { Ci } \\
\text { fractional } \\
\text { neutrons/sec } \\
\text { neutrons/sec } \\
\text { photons/sec } \\
\text { - }\end{array}$ \\
\hline
\end{tabular}


To specify the output of the tables listed in Table C-2, a number corresponding to those listed in Table C-3 must be placed on the OPTA, OPTF, and OPTL cards for each of the 24 tables. On each of these cards, 24 NOPTA, NOPTF, or NOPTL values must be entered. ORIGEN2 prints the tables by nuclide, by element, and in a summary table.

Table C-3. Specification of Output Tables on OPTA, OPTF and OPTL Cards

\begin{tabular}{|c|c|c|c|}
\hline \multirow{2}{*}{$\begin{array}{l}\text { NOPTA(I) } \\
\text { NOPTF(I) } \\
\text { NOPTL(I) }\end{array}$} & \multicolumn{3}{|c|}{ TABLE TYPE PRINTED } \\
\hline & NUCLIDE & ELEMENT & SUMMARY \\
\hline $\begin{array}{l}1 \\
2 \\
3 \\
4 \\
5 \\
6 \\
7 \\
8\end{array}$ & $\begin{array}{l}\text { Yes } \\
\text { Yes } \\
\text { Yes } \\
\text { No } \\
\text { Yes } \\
\text { No } \\
\text { No } \\
\text { No }\end{array}$ & $\begin{array}{l}\text { Yes } \\
\text { Yes } \\
\text { No } \\
\text { Yes } \\
\text { No } \\
\text { Yes } \\
\text { No } \\
\text { No }\end{array}$ & $\begin{array}{l}\text { Yes } \\
\text { No } \\
\text { Yes } \\
\text { Yes } \\
\text { No } \\
\text { No } \\
\text { Yes } \\
\text { No }\end{array}$ \\
\hline
\end{tabular}

ORIGEN2 specifies time units for decay intervals and others using the numbers listed in Table C-4 to represent a given unit.

Table C-4. Time Unit Designation

\begin{tabular}{|c|}
$1=$ seconds \\
$2=$ minutes \\
$3=$ hours \\
$4=$ days \\
$5=$ years \\
$6=$ stable \\
$7=10^{3}$ years $(\mathrm{kY})$ \\
$8=10^{6}$ years $(\mathrm{MY})$ \\
$9=10^{9}$ years (GY) \\
\end{tabular}




\section{C-3. Sample ORIGEN2 Input File Created by PreORG}

The following pages are a sample ORIGEN2 input file created by.PreORG for the Oak Ridge National Laboratory (ORNL) site for contact-handled transuranic (CH TRU) waste (ORNLCH93.U5). The spacing in the input file should be observed in all input files for ORIGEN2. 


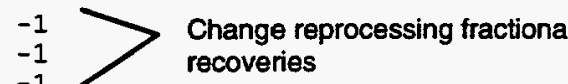

BAS WIPP BASELINE INVENTORY DATA

TIT ORNL STORED CONTACT HANDLED TRU WASTE

RDA ORNL

LIP $\quad \begin{array}{lllll}0 & 0 & 0 & \text { - Library Print Card }\end{array}$

LIB

PHO

RDA

INP

RDA

INP

RDA

INP

RDA

INP

RDA

INP

RDA

INP

RDA

INP

RDA

INP

RDA

INP

RDA

INP

RDA

INP

RDA

INP

RDA

INP

RDA

INP

RDA

INP

BAS

RDA

DEC

RDA

MOV

MOV

HED

HED

RDA

OPTA

OPTF

OPTL

RDA

OUT

RDA

DEC

RDA

MOV

MOV

HED

HED

RDA

$\begin{array}{llllllll}0 & 1 & 3 & 0 & 0 & 0 & 9 & 3\end{array}$ $\begin{array}{llll}101 & 102 & 103 & 10\end{array}$

INITIAL VALUES FOR: 1970

$\begin{array}{llllll}-1 & 1 & -1 & -1 & 1 & 1\end{array}$

INITIAL VALUES FOR: $1971^{\circ}$
$-2 \quad 1 \quad-1 \quad-1$ 1

INITIAL VALUES FOR: 1972

$\begin{array}{llllll}-3 & 1 & -1 & -1 & 1 & 1\end{array}$

INITIAI VALUES FOR: 1973

$\begin{array}{llllll}-4 & 1 & -1 & -1 & 1 & 1\end{array}$

INITIAL VALUES FOR: 1974

$\begin{array}{llllll}-5 & 1 & -1 & -1 & 1 & 1\end{array}$

INITIAI VALUES FOR: 1975

$\begin{array}{llllll}-6 & 1 & -1 & -1 & 1 & 1\end{array}$

INITIAL VALUES FOR: 1976

$\begin{array}{llllll}-7 & 1 & -1 & -1 & 1 & 1\end{array}$

INITIAL VALUES FOR: 1977 $\begin{array}{llllll}-8 & 1 & -1 & -1 & 1 & 1\end{array}$

INITIAL VALUES FOR: 1978

$\begin{array}{llllll}-9 & 1 & -1 & -1 & 1 & 1\end{array}$

INITIAL VALUES FOR: 1979

$\begin{array}{llllll}-10 & 1 & -1 & -1 & 1 & 1\end{array}$

INITIAL VALUES FOR: 1980

$\begin{array}{llllll}-11 & 1 & -1 & -1 & 1 & 1\end{array}$

INITIAL VALUES FOR: 1981

$\begin{array}{llllll}-12 & 1 & -1 & -1 & 1 & 1\end{array}$

INITIAL VALUES FOR: 1982

$\begin{array}{llllll}-13 & 1 & -1 & -1 & 1 & 1\end{array}$

INITIAL VALUES FOR: 1983

$\begin{array}{llllll}-14 & 1 & -1 & -1 & 1 & 1\end{array}$

INITIAL VALUES FOR: 1984

$\begin{array}{llllll}-15 & 1 & -1 & -1 & 1 & 1\end{array}$

DECAY INITIAL INVENTORIES TO: 1993 :orn]

DECAY YEAR 1970 TO 23.00 YRS

$23.00 \quad-1 \quad 15$ K

OUTPUT 1970 DECAY TO 1993 DATA

$\begin{array}{llll}1 & 2 & 0 & 1\end{array}$

$\begin{array}{llll}-1 & 1 & 0 & 1\end{array}$

11970

21993

PRINT NUCLIDE QUANTITIES IN CURIES

$6 \star 8 \quad 5 \quad 17 \star 8$

$6 * 8 \quad 5 \quad 17 * 8$

$6 * 8 \quad 5 \quad 17 * 8$

OUTPUT COMMANDS

$\begin{array}{llll}2 & 1 & -1 & 0\end{array}$

DECAY YEAR 1971 TO 22.00 YRS

$\begin{array}{lllll}22.00 & -2 & 2 & 5 & 1\end{array}$

OUTPUT 1971 DECAY TO 1993 DATA

$\begin{array}{llll}2 & 2 & 0 & 1\end{array}$

$\begin{array}{llll}-2 & 1 & 0 & 1\end{array}$

11971

21993

OPTA $6 * 8 \quad 5 \quad 17 * 8$

INPUT cards read initial nuclide inventories attached after STP card into vectors to be decayed out to 1993.

Library Card - Designates

Data Libraries Used By

ORIGEN2. For DECAY

calculations the $1,2,3$

designate the appropriate

libraries.

PRINT NUCLIDE QUANTITIES IN CURIES

Length of decay interval

Vector where initial nuclide concentrations are stored

Vector to store nuclide concentrations at end of decay interval
Determine continuous nuclide removal and feec rates if needed

ORG input file is

ornl_2.dat

- ORNL site

DECAY card instructs ORIGEN2 to periorm decay and accumulation calculations for nuclide concentrations stored in a given vector over a given interval:

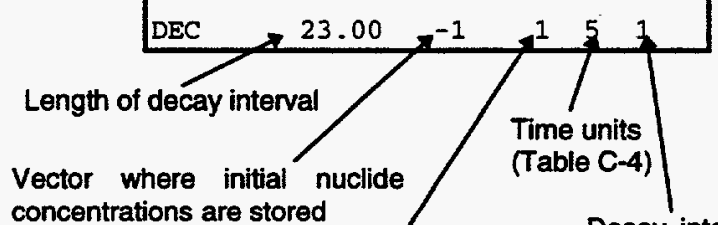

Decay interval length is independent of previous decay intervals used with prior DEC cards 


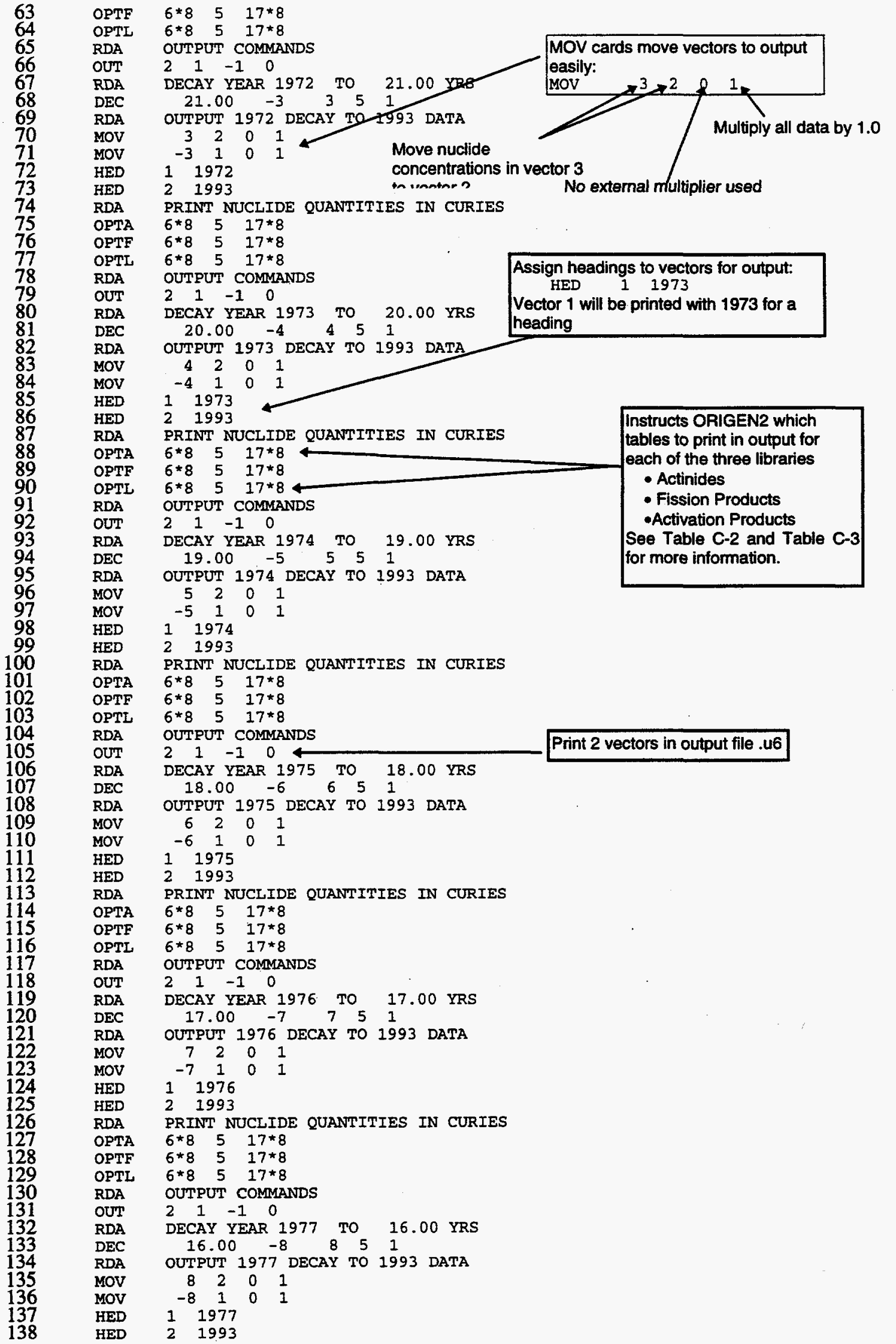




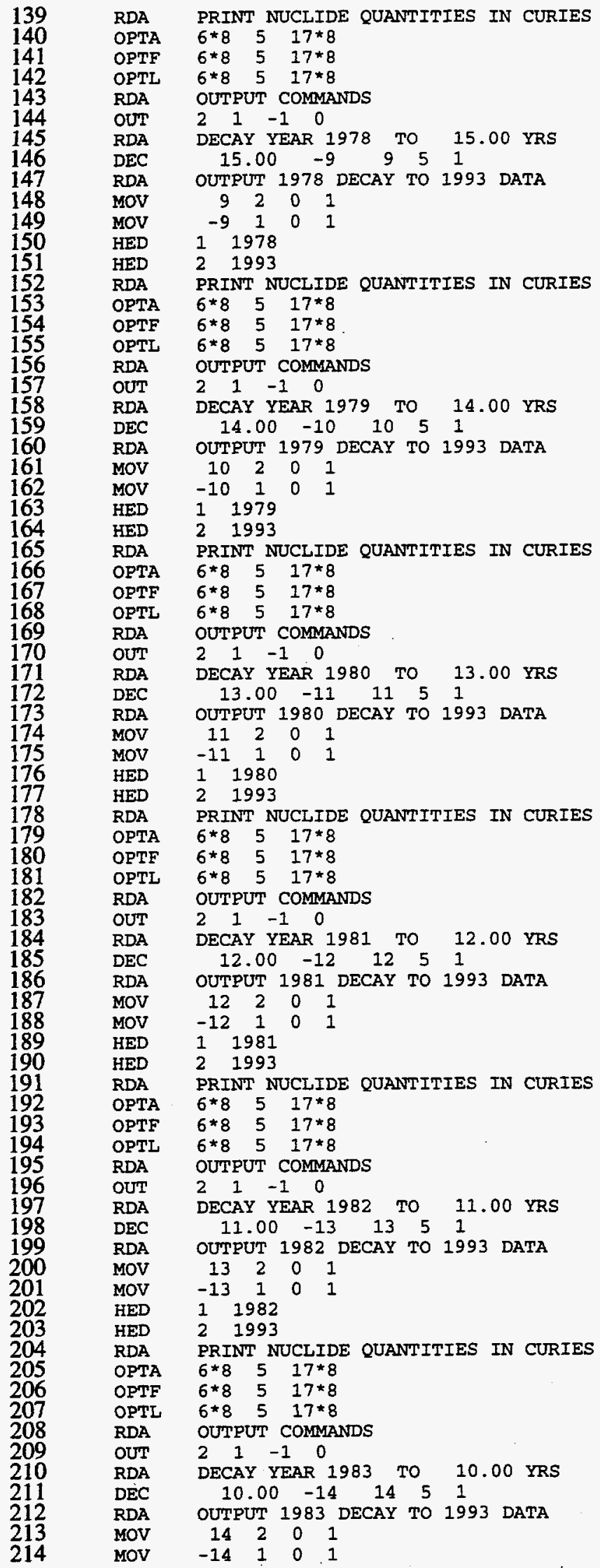


$\begin{array}{lll}982520 & .1860 \mathrm{E}-03 & 962420 \\ 932370 & .3703 \mathrm{E}+03 & 902320\end{array}$

$\begin{array}{lll}982520 & .1860 \mathrm{E}-03 & 962420 \\ 932370 & .3703 \mathrm{E}+03 & 902320\end{array}$

$\begin{array}{lll}982520 & .1860 \mathrm{E}-03 & 962420 \\ 932370 & .3703 \mathrm{E}+03 & 902320\end{array}$
First year of inventory file in grams

$.8277 E-04 \quad 922330$ $.1860 \mathrm{E}-08 \quad 952440 \quad .5477 \mathrm{E}-01$

Data that follows is read when new input

$.5262 \mathrm{E}+03 \quad 942400$ $.2565 E+03 \quad 922330$ $.2210 \mathrm{E}-02 \quad 982520$ $.6095 \mathrm{E}+01 \quad 932370$

$.8030 E+03$

$.1029 E-02$

$.3046 E+06$

$.5889 \mathrm{E}+02$

$.4051 \mathrm{E}+01$

$.8769 \mathrm{E}+01$

942400

952430

962440

922350

$.3902 \mathrm{E}+01$

$.5970 \mathrm{E}+02$

$.6227 \mathrm{E}-07$
$.1307 \mathrm{E}+03$

982520

902320

942400

922330

982520

922350

922330

982520

902320

952410

952410

972490

932370

922380

942410

952430

962440

922350

$.7726 E+01$ $.1571 E+02$

$.2121 \mathrm{E}-02$

$.4583 E+04$

$2059 \mathrm{E}+01$ $.1857 \mathrm{E}+02$

$.5193 \mathrm{E}+01$

$.7681 \mathrm{E}+02$

$.1576 \mathrm{E}+01$

$.2263 \mathrm{E}+03$

$.9952 \mathrm{E}-01$

$.1069 \mathrm{E}+03$

$.1009 \mathrm{E}+04$

$.3348 \mathrm{E}-03$

$.9667 \mathrm{E}+03$

$.9443 E+00$

$.3199 \mathrm{E}-05$

$.1988 \mathrm{E}+04$

$.5531 \mathrm{E}-04$

$.1759 \mathrm{E}+03$

$.1389 \mathrm{E}+05$

\begin{abstract}
$.8117 E+03$
\end{abstract}
$.1505 \mathrm{E}-03$

$.3425 E+00$

$.3225 \mathrm{E}+03$ in oml__ 2.dat input card is encountered.

$.1326 \mathrm{E}+01$

Indicates end of card of data

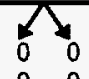

$.5975 \mathrm{E}-03$

$.3816 \mathrm{E}-04$

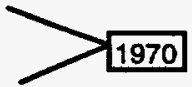

1970
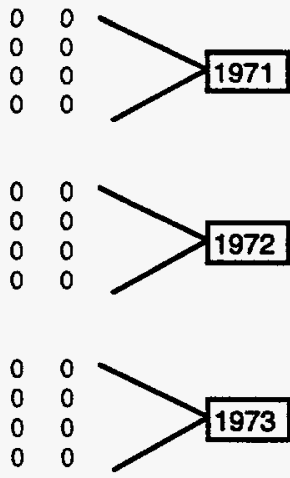

902320
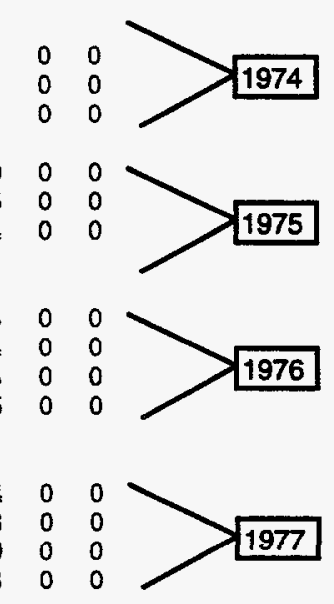
Sample ORIGEN2 Input File Created by PreORG

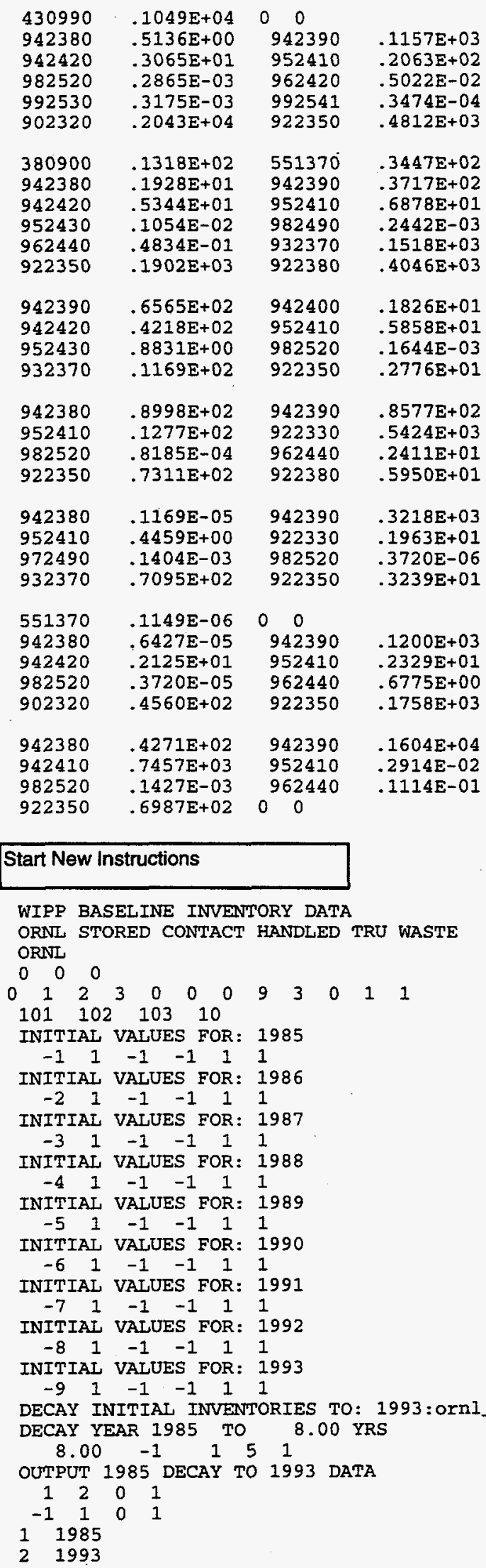


RDA PRINT NUCLIDE QUANTITIES IN CURIES

$\begin{array}{lll}6 \star 8 & 5 & 17 \star 8\end{array}$

$\begin{array}{llll}\text { OPTF } & 6 * 8 & 5 & 17 * 8\end{array}$

OPTL $6 * 8 \quad 5 \quad 17 * 8$

RDA OUTPUT COMMANDS

OUT $2 \begin{array}{llll}2 & 1 & -1 & 0\end{array}$

RDA DECAY YEAR 1986 TO 7.00 YRS

DEC $\quad 7.00 \quad-2 \quad 2 \quad 5 \quad 1$

RDA OUTPUT 1986 DECAY TO 1993 DATA

MOV $\quad 2 \quad 2 \quad 001$

MOV $-2 \quad 1 \quad 0 \quad 1$

HED 11986

HED 21993

RDA PRINT NUCLIDE QUANTITIES IN CURIES

OPTA $6 * 8 \quad 5 \quad 17 * 8$

OPTF $\quad 6 * 8 \quad 5 \quad 17 * 8$

OPTL $6 * 8 \quad 5 \quad 17 * 8$

RDA OUTPUT COMMANDS

OUT $2 \begin{array}{llll}2 & 1 & -1 & 0\end{array}$

RDA DECAY YEAR 1987 TO 6.00 YRS

DEC $\quad 6.00 \quad-3 \quad 3 \quad 5 \quad 1$

RDA OUTPUT 1987 DECAY TO 1993 DATA

MOV $\quad 3 \quad 25001$

MOV

HED

RDA

OPTA

OPTF

OPTL

RDA

OUT

RDA

DEC

RDA

MOV

MOV

HED

HED

RDA

OPTA

OPTF

OPTI

RDA

OUT

RDA

DEC

RDA

MOV

MOV

HED

HED

RDA

OPTA

OPTF

OPTL

RDA

OUT

RDA

DEC

RDA

MOV

MOV

HED

HED

RDA

OPTA

OPTF

OPTL

RDA

$\begin{array}{llll}-3 & 1 & 0 & 1\end{array}$

11987

$\begin{array}{ll}2 & 1993\end{array}$

PRINT NUCLIDE QUANTITIES IN CURIES

$6 * 8 \quad 5 \quad 17 * 8$

$6 * 8 \quad 5 \quad 17 * 8$

$6 * 8 \quad 5 \quad 17 * 8$

OUTPUT COMMANDS

$\begin{array}{llll}2 & 1 & -1 & 0\end{array}$

DECAY YEAR 1988. TO 5.00 YRS

$$
5.00-4 \quad 4 \quad 5 \quad 1
$$

OUTPUT 1988 DECAY TO 1993 DATA

$\begin{array}{rrrr}4 & 2 & 0 & 1 \\ -4 & 1 & 0 & 1\end{array}$

11988

21993

PRINT NUCLIDE QUANTITIES IN CURIES

$6 * 8 \quad 5 \quad 17 \star 8$

$6 * 8 \quad 5 \quad 17 * 8$

$6 \star 8 \quad 5 \quad 17 \star 8$

OUTPUT COMMANDS

$\begin{array}{llll}2 & 1 & -1 & 0\end{array}$

DECAY YEAR 1989 TO 4.00 YRS $4.00 \quad-5 \quad 5 \quad 5 \quad 1$

OUTPUT 1989 DECAY TO 1993 DATA

$\begin{array}{llll}5 & 2 & 0 & 1\end{array}$

$\begin{array}{llll}-5 & 1 & 0 & 1\end{array}$

11989

21993

PRINT NUCLIDE QUANTITIES IN CURIES

$6 * 8 \quad 5 \quad 17 * 8$

$6 * 8 \quad 5 \quad 17 * 8$

$6 * 8 \quad 5 \quad 17 * 8$

OUTPUT COMMANDS

$\begin{array}{llll}2 & 1 & -1 & 0\end{array}$

DECAY YEAR 1990 TO 3.00 YRS $\begin{array}{lllll}3.00 & -6 & 6 & 5 & 1\end{array}$

OUTPUT 1990 DECAY TO 1993 DATA

$\begin{array}{llll}6 & 2 & 0 & 1\end{array}$

$\begin{array}{llll}-6 & 1 & 0 & 1\end{array}$

11990

21993

PRINT NUCLIDE QUANTITIES IN CURIES

$6 * 8 \quad 5 \quad 17 * 8$

$6 * 8 \quad 5 \quad 17 * 8$

$6 * 8 \quad 5 \quad 17 * 8$

OUTPUT COMMANDS

OUT $\quad 2 \quad 1 \quad-1 \quad 0$

RDA

DEC

RDA

MOV

$\begin{array}{llllll}430 & \text { MOV } & -7 & 1 & 0 & 1\end{array}$

DECAY YEAR 1991 TO 2.00 YRS

OUTPUT 1991 DECAY TO 1993 DATA

$\begin{array}{llll}7 & 2 & 0 & 1\end{array}$ 
Sample ORIGEN2 Input File Created by PreORG

ornich93.u5

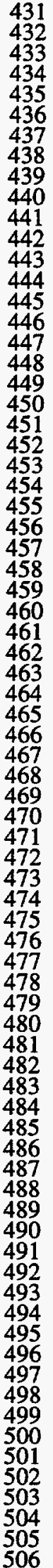

HED $\quad 1 \quad 1991$

HED 201993

RDA PRINT NUCLIDE QUANTITIES IN CURIES

OPTA $6 * 8 \quad 5 \quad 17 \star 8$

OPTF $6 * 8 \quad 5 \quad 17 * 8$

OPTL $\quad 6 * 8 \quad 5 \quad 17 * 8$

RDA OUTPUT COMMANDS

OUT $\quad 2 \quad \begin{array}{llll}1 & -1 & 0\end{array}$

RDA DECAY YEAR 1992 TO 1.00 YRS

DEC $1.00 \quad-8 \quad 8 \quad 5 \quad 1$

RDA OUTPUT 1992 DECAY TO 1993 DATA

MOV $\quad \begin{array}{rrrr}8 & 2 & 0 & 1\end{array}$

MOV $\quad \begin{array}{lllll}-8 & 1 & 0 & 1\end{array}$

HED 111992

HED 21993

RDA PRINT NUCLIDE QUANTITIES IN CURIES

OPTA $6 * 8 \quad 5 \quad 17 * 8$

OPTF $6 * 8 \quad 5 \quad 17 * 8$

OPTL $\quad 6 * 8 \quad 5 \quad 17 * 8$

RDA OUTPUT COMMANDS

OUT $2 \begin{array}{llll} & 1 & -1 & 0\end{array}$

RDA DECAY YEAR 1993 TO .00 YRS

DEC $\quad .00 \quad-9 \quad 9051$

RDA OUTPUT 1993 DECAY TO 1993 DATA

MOV $\quad 9 \begin{array}{llll}2 & 0 & 1\end{array}$

MOV $\quad-9 \quad 1 \quad 0 \quad 1$

HED 11993

HED $\quad 2 \quad 1993$

RDA PRINT NUCLIDE QUANTITIES IN CURIES

OPTA $6 * 8 \quad 5 \quad 17 * 8$

OPTF $\quad 6 * 8 \quad 5 \quad 17 * 8$

OPTL $\quad 6 * 8 \quad 5 \quad 17 * 8$

RDA OUTPUT COMMANDS

$\begin{array}{llll}2 & 1 & -1 & 0\end{array}$

END

942380

922330

962420

882260

380900

942380

952410

982520

932370

611470

942380

942410

922330

932370

922380

942380

942410

952430

962440

882260

942380

942410

922330

922320

942380

952410

982520

882260

942390

982490

962440

922380

260590

$\$ 90900$
$.1209 \mathrm{E}+02$

$.8958 \mathrm{E}+03$

$.3025 E-04$

.1012E-03

$.5859 \mathrm{E}-03$

$.1250 \mathrm{E}-02$

$.2434 \mathrm{E}+01$

$.1602 \mathrm{E}-04$

$.9748 \mathrm{E}+00$

$.1186 \mathrm{E}-04$

$.2179 E+01$

$.4816 \mathrm{E}+01$

$.4588 \mathrm{E}+02$

$4966 E+02$

$.3600 E+03$

$.5843 \mathrm{E}-02$

$.2719 \mathrm{E}-04$

$.5018 \mathrm{E}+00$

$.6132 \mathrm{E}-03$

$.3035 E-02$

$.3506 \mathrm{E}-03$

$.5340 \mathrm{E}-03$

$.4123 \mathrm{E}+03$

$.5094 \mathrm{E}-03$

$.1186 \mathrm{E}-02$

$.2049 \mathrm{E}+00$

$.1382 \mathrm{E}-05$

.1770 E-02

$.6453 E+01$

$.4884 \mathrm{E}-03$

$.1154 \mathrm{E}-01$

$.5058 \mathrm{E}+02$

$8124 E-0$

$\begin{array}{ll}.8124 E-04 & 270600 \\ .5508 E-11 & 551370\end{array}$

942390

952430

962440

922350

551370

942390

922330

962420

922320

00

942390

942420

982520

922320

00

942390

952410

982490

962480

00

942390

942420

982520

922340

942390

922330

962440

922360

952410

982520

882260

0
Terminate ORIGEN2 execution $\begin{array}{lllll}2716 \mathrm{E}+04 & 952410 \quad .6732 \mathrm{E}+01 & 0 & 0\end{array}$

$\begin{array}{llll}922380 & .5950 \mathrm{E}+01 & 0 & 0\end{array}$

$.9191 \mathrm{E}-03 \quad 0 \quad 0$

$.4232 E+02 \quad 942400$

$2149 \mathrm{E}+03 \quad 952430$

$1513 E-06 \quad 962440$

$.8224 \mathrm{E}-02 \quad 922380$

$.4843 E+03$

$5109 \mathrm{E}+00$

$.4595 \mathrm{E}-04$

$.5608 \mathrm{E}-02$

$.1706 \mathrm{E}+02$

$7519 \mathrm{E}-01$

2442E-02

$4517 \mathrm{E}+01$

$1340 \mathrm{E}+02$

$.6025 \mathrm{E}-02$

$.1860 \mathrm{E}-04$

9604E-01

$1292 \mathrm{E}+02$

$5993 E+02$

$.1199 \mathrm{E}+01$

$3092 E+00$

2069E-02

2046E-04

$1608 \mathrm{E}+01$

$.2650 \mathrm{E}-08$

$.9191 \mathrm{E}-03$
942400

952410

962440

922340

942400

922330

982520

932370

942400

952410

962440

922350

942410

952430

932370

00

922330

962420

902320

$.1677 E+02 \quad 0 \quad 0$

$.1004 \mathrm{E}+00 \quad 0 \quad 0$

$\begin{array}{lll}.1385 E-02 & 0 & 0 \\ .6456 E+03 & 0 & 0\end{array}$

$.1734 E+03 \quad 0 \quad 0$

7548E-02 00

$.5712 E-03 \quad 0 \quad 0$

$.1601 E-0200$

4390E-02 $0 \quad 0$

$.2294 E+03 \quad 0 \quad 0$

$.8222 \mathrm{E}-04 \quad 0 \quad 0$

$.2838 \mathrm{E}+02 \quad 0 \quad 0$

$.3516 \mathrm{E}+01 \quad 0 \quad 0$

$.3148 \mathrm{E}+00 \quad 0 \quad 0$

$.3338 E-04 \quad 0 \quad 0$

$5044 E+0300$

$.7078 \mathrm{E}+01 \quad 0 \quad 0$

9283E-03 00

$.1986 \mathrm{E}+03$ 0 0

$.2066 \mathrm{E}+02 \quad 0 \quad 0$

$.2118 \mathrm{E}-04 \quad 0 \quad 0$

$.3739 E+03 \quad 0 \quad 0$

$380900 \quad .2197 \mathrm{E}-07 \quad 0 \quad 0$

$611470 \quad .2156 \mathrm{E}-07 \quad 0 \quad 0$ 
Appendix C: ORIGEN2 Command Descriptions and Sample ORIGEN2 Input File Created by PreORG

507
508
509
510
511
512
513
514
515
516
517
518
519
520

Sample ORIGEN2 Input File Created by PreORG

ornlch93.u5

942380

942410

962440

922350

270600

280630

942410

922330

922360
972490
$.4394 E+01$

$3262 \mathrm{E}+01$

$.3932 \mathrm{E}-02$

E $+02 \quad 932370$

$.1078 E-04 \quad 380900$

$1798 \mathrm{E}-05 \quad 0 \quad 0$

$.2767 \mathrm{E}+02 \quad 942420$

$1178 \mathrm{E}+01 \quad 922340$

$\begin{array}{ll}.4273 E-08 & 982490\end{array}$
$.1673 \mathrm{E}+02$

$9559 \mathrm{E}+00$
$1465 \mathrm{E}-04$

$.1166 \mathrm{E}+03$

$.1036 \mathrm{E}+01$

$.8935 E-04$

$.4908 \mathrm{E}+04$

$4349 E+01$

$.1365 \mathrm{E}+04$

$1048 \mathrm{E}+03$

Indicates data library 1. Activation Products

2 - Actinides

3 - Fission Products

C-14 
Sample Index File Created by PreORG: sample.ind

INDEX FILENAME: sample
$8 / 15 / 1995$ at 0842

ORIGEN2 INPUT FILE YEARS OF INVENTORY SITE NAME DECAY ALL YEARS TO:

hanfch93.u5 $\quad 1970$ thru $1993 \quad$ HANFORD 1993

Enriched U Atom Fractions: U234 $=.00$ U235 $=.93$ U238 $=.01$

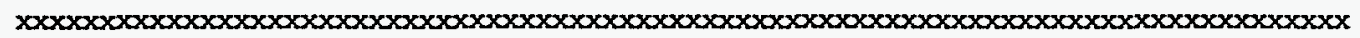

hanfrh93.u5 1970 thru $1988 \quad$ HANFORD 1993

Enriched U Atom Fractions: U234 $=.00$ U235 $=.93$ U238 $=.06$

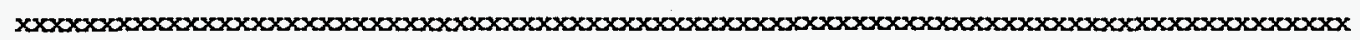

inelch93.u5 1970 thru $1992 \quad$ INEL 1993

Enriched U Atom Fractions: U234= .00 U235= .07 U238 $=.93$

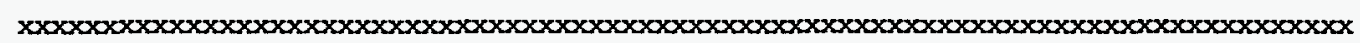

inelrh93.u5 1977 thru 1993 INEL 1993

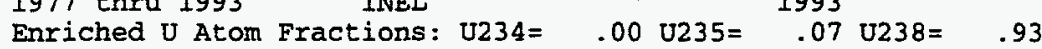

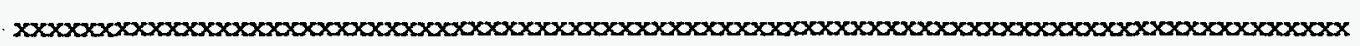

$\begin{array}{llll}\text { ornlch93.u5 } 1970 \text { thru } 1993 \quad \text { ORNL } & 1993\end{array}$

Enriched U Atom Fractions: U234 $=\quad .00$ U235 $=\quad .07$ U238 $=.93$

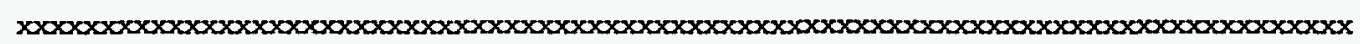

ornlrh93.u5 1970 thru $1993 \quad$ ORNL 1993

Enriched U Atom Fractions: U234 $=.00$ U235 $=.07$ U238 $=.93$

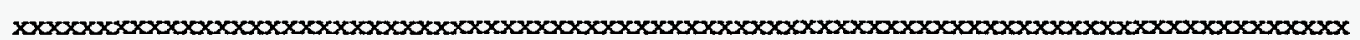

$\begin{array}{llll}\text { ornlbug3.u5 } & 1972 \text { thru } 1974 \quad \text { ORNL } & 1993\end{array}$

Enriched U Atom Fractions: U234 $=.00$ U235 $=\quad .07$ U238 $=.93$

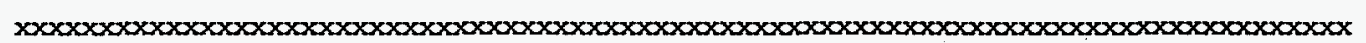


Appendix C: ORIGEN2 Command Descriptions and Sample ORIGEN2 Input File Created by PreORG

Sample ORIGEN2 Input File Created by PreORG

ornlch93.u5

Intentionally Left Blank 
APPENDIX D: OR1_PREORG.FOR PROGRAM LISTING

D-1 
Intentionally Left Blank 
PRE0001

PROGRAM PREORG

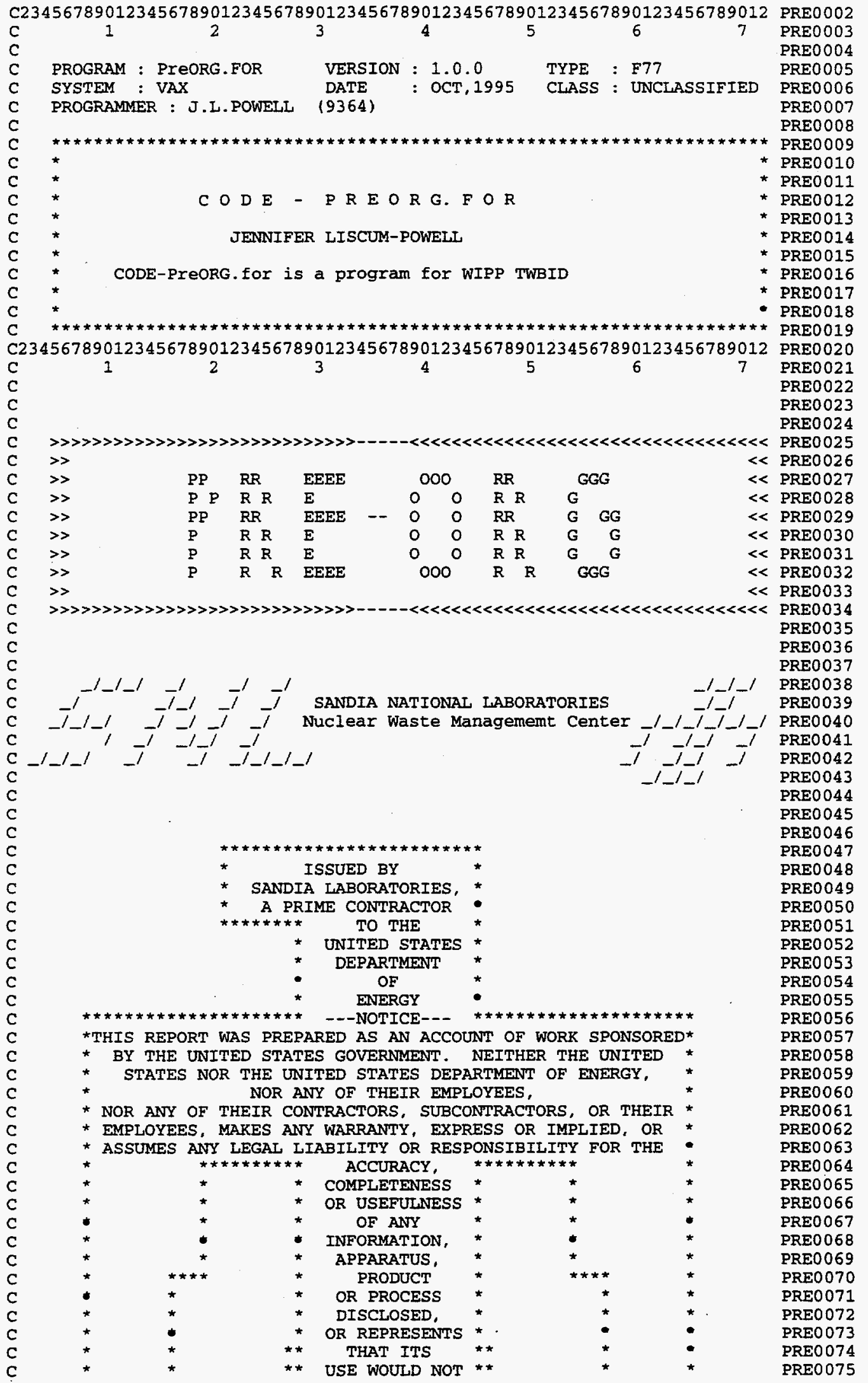

D-3 


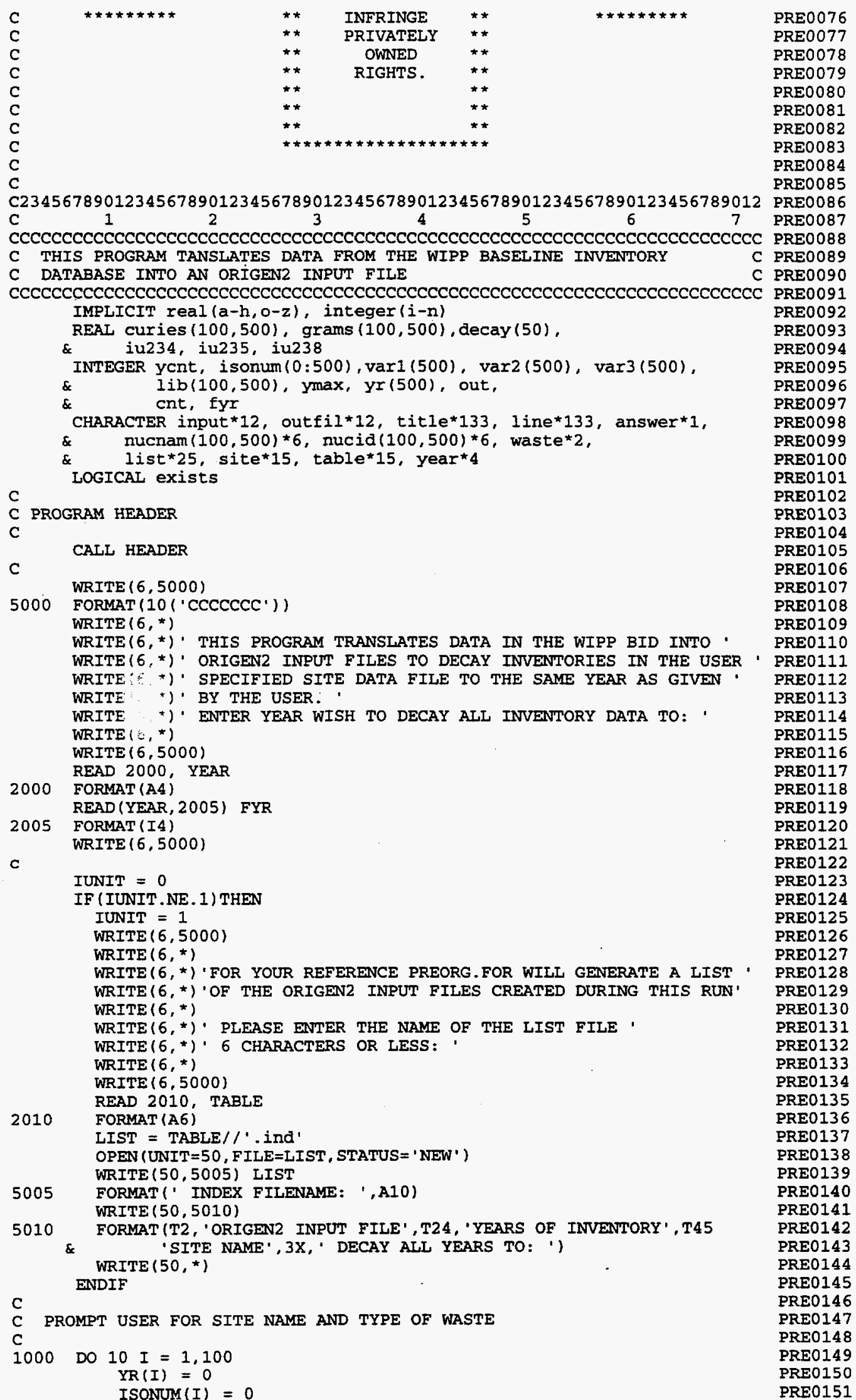




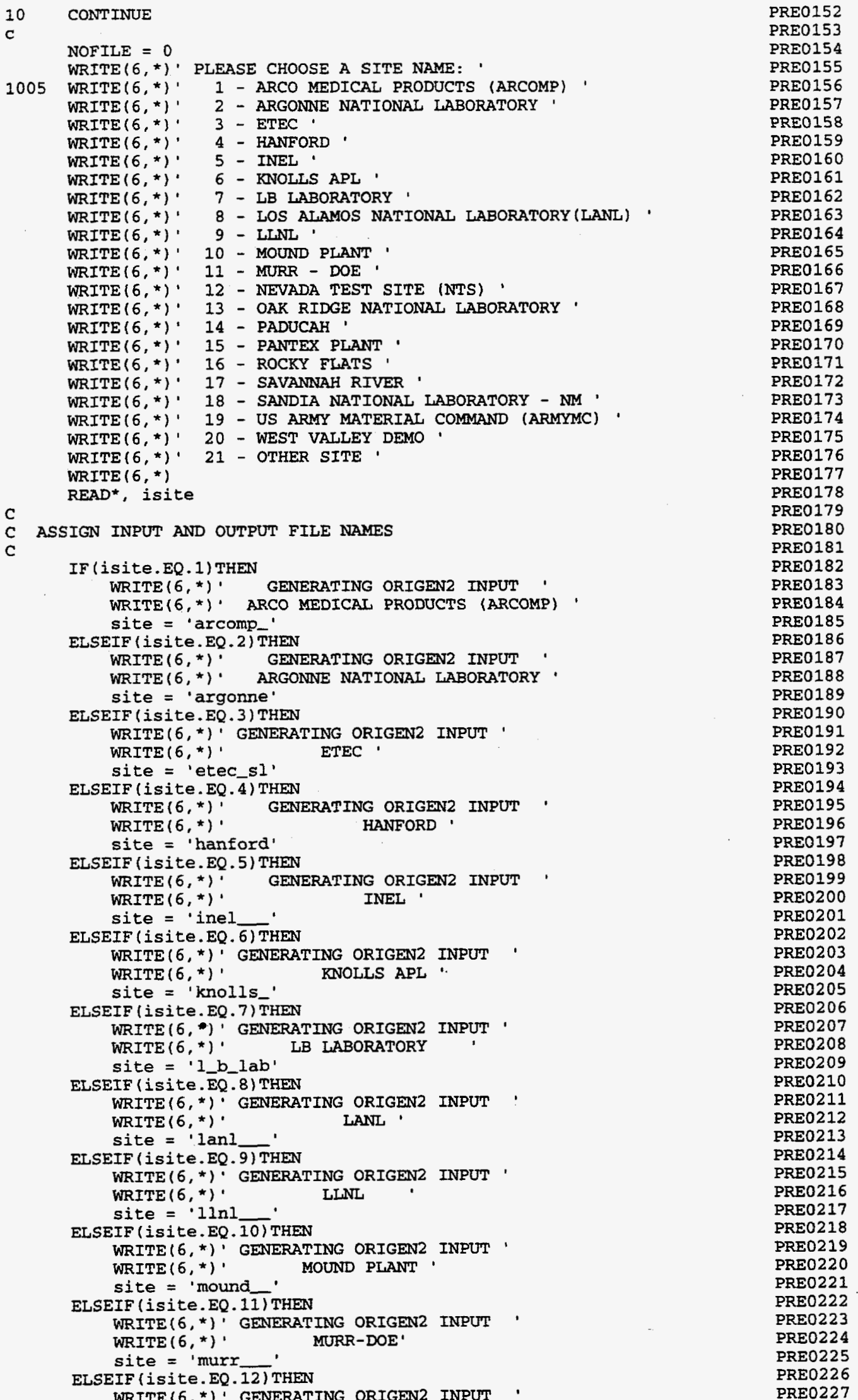




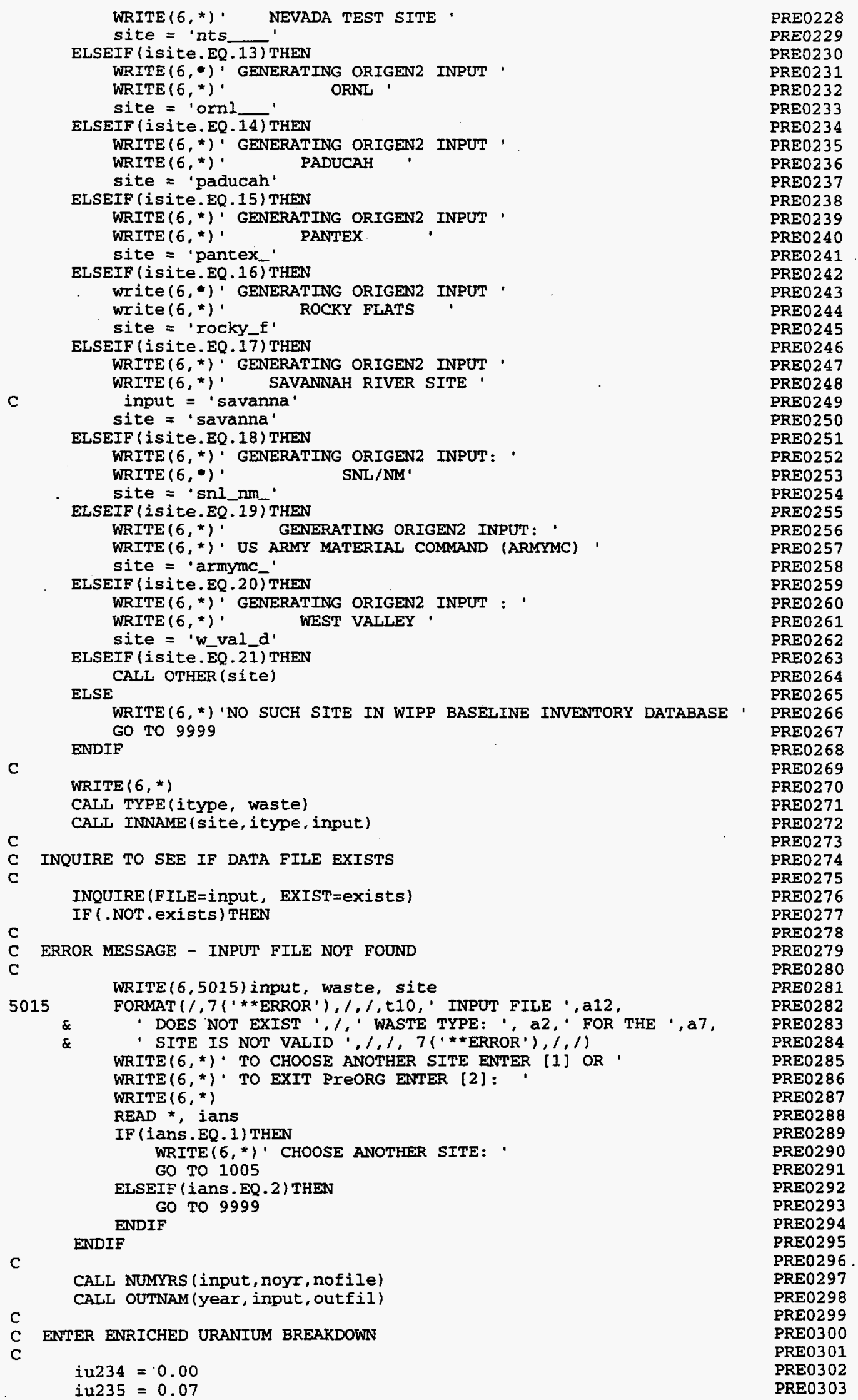


iu238 $=0.93$

WRITE $(6, *)$

WRITE $(6, *)$, DEFAULT ENRICHED URANIUM BREAKDOWN IS:

WRITE $(6, *)$. U234 - 0.00 '

WRITE $(6, *) \cdot \quad$ U235 -0.07

WRITE $(6, *)$ U238 -0.93 ,

WRITE $(6,5020)$ site

FORMAT $(2 \mathrm{x}$, ' DO YOU

\& $215, ;$ ? ')

WRITE $(6, *)$ ' Enter $y$ (yes) or $n$ (no): '

READ 5025, ANSWER

5025 FORMAT (AI)

WRITE $\left(6,{ }^{*}\right)$

WRITE $(6,5000)$

IF (answer. EQ. ' $y$ ' . OR. answer. EQ. ' $Y$ ') THEN

WRITE $(6, *)$ PLEASE ENTER ENRICHED URANIUM BREAKDOWN AS * PREO319

WRITE $(6, *)$, ATOM FRACTIONS: ' PRE0320

WRITE $(6, *)$ U234 ='

READ*, iu234

$\operatorname{WRITE}(6, *) \cdot \quad \mathrm{U} 235=$

READ*, iu235

WRITE $(6, \star)$ '

$\mathrm{U} 238=$

READ*, iu238

ENDIF

WRITE $(6, *)$

$\operatorname{WRITE}(6,5000)$

WRITE $(6, *)$

C OPEN INPUT AND OUTPUT FILES

c

OPEN (UNIT $=10$, FILE= INPUT , STATUS = ' OLD' )

OPEN (UNIT = 15, FILE=OUTFIL, STATUS= ' NEW' )

C

READ INPUT DATA FROM FILE

C

1015 yent $=0$

icnt $=1$

$c$

$1020 \operatorname{READ}(10,2020$, END $=8000)$ line

2020 FORMAT (A133)

c write $(6, *)$ line

C

IF (icnt.EQ.1) THEN

title = line

icnt $=2$

GO TO 1020

ENDIF

C

IF (1ine $(1: 2) . E Q \cdot \cdot^{\prime} 19^{\prime}$. OR. Iine $(1: 2)$, EQ.' $\left.20^{\prime}\right)$ THEN

$y c n t=y c n t+1$

isonum (yent) $=0$

READ (LINE, 2025) yr (ycnt)

2025

FORMAT (I4)

icnt $=$ icnt +1

ENDIF

GO TO 1020

IF (Iine (3:7) EQ.'ZZZZZ')GO TO 1020

C

READ ISOTOPES AND ACTIVITIES IN INVENTORY FOR PRESENT YEAR AND SITE

isonum (ycnt) = isonum (yent) +1

READ (LINE, 2030) nucnam (ycnt, isonum (ycnt)), curies (ycnt, isonum (ycnt))

2030 FORMAT (T3, A6, T41, E10.4)

c write $(6,2030)$ nucnam(ycnt, isonum (ycnt)),

$\delta$ curies (ycnt, isonum (ycnt))

DEPLETED URANIUM - ASSUME $100 \%$ U-238

IF (nucnam (ycnt, isonum (ycnt) ) (1:4) . EQ. ' UDPL ' ) THEN nucnam (ycnt, isonum (ycrt)) = 'U238' GO TO 1020

C

C NATURAL URANIUM - ATOM\& FROM CHART OF NUCLIDES

ELSEIF (nucnam (ycnt, isonum (ycnt)) (1:4). EQ. ' UNAT' ) THEN

PRE0321

PREO322

PRE0323

PRE0324

PRE0325

PRE0326

PRE0 327

PRE0328

PRE0329

PRE0330

PRE0331

PRE0332

PRE0333

PRE0334

PRE0335

PRE0336

PRE0337

PRE0338

PRE0339

PRE0340

PRE0 341

PRE0 342

PRE0343

PRE0344

PRE0345

PRE0346

PRE0347

PRE0348

PRE0349

PRE0350

PRE0351

PRE0352

PRE0353

PRE0354

PRE0355

PRE0356

PRE0357

PRE0358

PRE0359

PRE0360

PRE0361

PRE0 362

PRE0363

PRE0364

PRE0365

PRE0 366

PRE0367

PRE0368

PRE0369

PRE0370

PRE0371

PRE0 0372

PRE0 373

PRE0374

PRE0375

PRE0376

PRE0377

PRE0378

PRE0379 


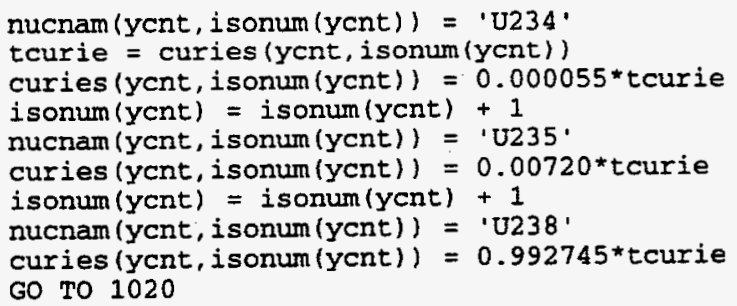

C

C

ENRICHED URANIUM - 938 U-235 AND $7 \%$ U-238

ELSEIF (nuenam (yent, i sonum (yent) ) (1:4) . EQ. ' UENR') THEN tcurie $=\operatorname{curies}(y c n t$, isonum (ycnt)) nucnam (ycnt, isonum (ycnt)) $=\mathrm{U}^{\mathrm{U}} 2 \mathrm{5}^{\circ}$ curies (ycnt, isonum $(y \subset n t))=i u 235^{*}$ tcurie isonum (ycnt) $=$ isonum (ycnt) +1 nucnam (ycnt, isonum (ycnt)) = 'U238. curies (ycnt, isonum (ycnt)) = iu238*tcurie IF (iU234.NE.0) THEN

isonum (ycnt) $=$ isonum $(y c n t)+1$

nucnam (ycnt, isonum (ycnt)) $=\mathrm{v} 238^{\circ}$

curies (ycnt, isonum (ycnt)) $=$ iu2 $38^{*}$ tcurie ENDIF

GO TO 1020

FOR UNKNOWN NUCLIDES IGNORE ACTIVITIES IN CURIES

ELSEIF (nucnam (ycnt, isonum (ycnt) ) (1:4) . EQ. ' UNKN') THEN isonum $(y c n t)=$ isonum $(y c n t)-1$ GO TO 1020

FOR P0209 - NO DATA IN ORIGEN2 LIBRARIES

ELSEIF (nucnam (ycnt, isonum (ycnt)) (1:5) .EQ. ' PO209') THEN isonum $(y \subset n t)=$ isonum $(y \subset n t)-1$ GO TO 1020

$7 / 26 / 95$ FOR MIXED ACTIVATION PRODUCTS WHOSE ISOTOPIC COMPOSITION IS NOT GIVEN BREAKDOWN PROVIDED BY SAYAN CHAKRABORTI, SAIC IS USED TO DETERMINE THE ISOTOPIC COMPOSITIONS (FROM HANFORD)

ELSEIF (nucnam (ycnt, isonum (ycnt)) $(1: 6)$.EQ. ' TBGMAP') THEN tcurie = curies (yont, isonum (ycnt) nucnam (yent, isonum (ycnt)) = BA137M curies (yent, isonum $(y \operatorname{con} t))=0.0097 *$ tcurie isonum (yent) $=$ isonum $(y c n t)+1$ nucnam (ycnt, isonum (ycnt)) $={ }^{\prime} \mathrm{C} 14$ ' curies (ycnt, isonum (ycnt)) $=0.01^{\star}$ tcurie isonum (ycnt) $=$ isonum (ycnt) +1 nuenam (yent, isonum (ycnt)) $={ }^{\prime} \mathrm{CO} 058^{\prime}$ curies (ycnt, isonum (ycnt)) $=0.01 *$ tcurie isonum (ycnt) $=$ isonum (ycnt) +1 nucnam (ycnt, isonum (ycnt)) $=' \mathrm{c} 060^{\prime}$ curies (ycnt, isonum (ycnt)) $=0.67 *$ tcurie isonum (ycnt) = isonum (yent) +1 nucnam (yent, isonum (ycnt)) $={ }^{\prime} \operatorname{CS} 137$ ' curies (ycnt, isonum (yent)) $=0.021241 *$ tcurie isonum (ycnt) $=$ isonum (ycnt) +1 nucnam (ycnt, isonum (ycnt)) = 'EU152' curies (ycnt, isonum (ycnt)) $=0.01 *$ tcurie isonum (ycnt) $=$ isonum (ycnt) +1 nucnam (ycnt, isonum (yCnt)) $=1 \mathrm{EU154}$ curies (ycnt, isonum $(y c n t))=0.085 *$ tcurie isonum (ycnt) $=$ isonum (ycnt) +1 nucnam (ycnt, isonum (ycnt)) = 'EU155' curies (ycnt, isonum (ycnt)) $=0.085^{*}$ tcurie isonum (ycnt) $=i$ sonum (ycnt) +1 nucnam (ycnt, isonum (ycnt)) = FE59 curies (ycnt, i sonum (ycnt)) $=0.04 *$ tcurie isonum (ycnt) $=i$ sonum $(y<n t)+1$ nucnam (ycnt, isonum (ycnt)) = 'MN54 curies (ycnt, isonum (ycnt)) $=0.07 *$ tcurie GO TO 1020

PRE0384

PRE0385

PRE0386

PRE0387

PRE0388

PRE0389

PRE0390

PRE0391

PRE0 392

PRE0393

PRE0394

PRE0395

PRE0396

PRE0397

PRE0398

PRE0399

PRE0400

PRE0401

PRE0402

PRE0403

PRE0 0404

PRE0405

PRE0406

PRE0407

PRE0408

PRE0409

PRE0410

PRE0411

PRE0 412

PRE 0413

PRE0414

PRE0415

PRE0416

PRE0 417

PRE0418

PRE0419

PRE0 420

PRE0421

PRE0422

PRE0423

PRE0424

PRE0425

PRE0426

PRE0 427

PRE0428

PRE0429

PRE0430

PRE0431

PRE0 432

PRE0 433

PRE0434

PRE0 435

PRE0436

PRE 0437

PRE0438

PRE0 439

PRE0440

PRE0441

PRE0442

PRE0443

PRE0444

PRE0445

PRE0 0446

PRE0447

PRE0448

PRE0449

PRE0450

PRE0451

PRE0452

PRE0453

PRE0454

PRE0 455 
C 7/26/95 FOR MIXED ACTIVATION PRODUCTS WHOSE ISOTOPIC COMPOSITION

C IS NOT GIVEN BREAKDOWN PROVIDED BY SAYAN CHAKRABORTI, SAIC IS USED TO DETERMINE THE ISOTOPIC COMPOSITIONS (FROM HANFORD)

ELSEIF (nucnam (ycnt, isonum (ycnt)) $(1: 6)$.EQ. 'TBGMFP') THEN tcurie $=\operatorname{curies}(y c n t$, isonum $(y c n t)$ ) nucnam (ycnt, isonum (ycnt)) = AG110M'

curies (ycnt, isonum (ycnt)) $=5.02 \mathrm{E}-6^{*}$ tcurie isonum (ycnt) $=$ isonum (ycnt) +1

nucnam (ycnt, isonum (ycnt)) = 'AG110'

curies (ycnt, isonum (ycnt)) $=2.507 E-8 *$ tcurie isonum (ycnt) $=$ isonum (ycnt) +1

nucnam (ycnt, isonum (ycnt)) $={ }^{\prime} B A 137 \mathrm{M}$ '

curies (ycnt, isonum (ycnt)) $=0.01848 *$ tcurie

isonum (ycnt) $=$ isonum (ycnt) +1

nucnam (ycnt, isonum (ycnt)) $={ }^{\prime} \operatorname{CD} 113 \mathrm{M}$ '

curies (ycnt, isonum (ycnt)) $=5.0 \mathrm{E}-10^{*}$ tcurie

isonum (ycnt) $=$ isonum (ycnt) +1

nucnam (ycnt, isonum (ycnt)) $=$ 'CE144'

curies (ycnt, isonum (ycnt)) $=0.2784 *$ tcurie

isonum (ycnt) $=$ isonum (ycnt) +1

nucnam (ycnt, isomum (ycnt)) $=\cdot \operatorname{cs} 134$ '

curies (ycnt, isonum (ycnt)) $=5.557 \mathrm{E}-4 *$ tcurie

isonum (ycnt) $=$ isonum (ycnt) +1

nucnam (ycnt, isonum $(y c n t))={ }^{\prime} \operatorname{cs} 135^{\circ}$

curies (ycnt, isonum (ycnt)) $=5.750 \mathrm{E}-8 *$ tcurie

isonum (ycnt) $=$ isonum (ycnt) +1

nucnam (ycnt, isonum (ycnt)) $=\cdot \operatorname{cs} 137$.

curies (ycnt, isonum (ycnt)) $=2.009 \mathrm{E}-2 *$ tcurie

isonum (ycnt) $=$ isonum (ycnt) +1

nucnam (ycnt, isonum (ycnt)) = 'EU152.

curies (ycnt, isonum (ycnt)) $=3.0 \mathrm{E}-7 *$ tcurie

isonum (ycnt) $=i$ sonum $(y \subset n t)+1$

nucnam (ycnt, isonum (ycnt)) = EU154.

curies (ycnt, isonum (ycnt)) $=3.038 \mathrm{E}-5 *$ tcurie

isonum (ycnt) $=$ isonum (ycnt) +1

nucnam (ycnt, isonum (ycnt)) = $\mathrm{EU} 155^{\circ}$

curies (ycnt, isonum (ycnt) $)=7.353 \mathrm{E}-4 *$ tcurie

isonum (ycnt) $=$ isonum $(y \circ n t)+1$

nucnam (ycnt, isonum (ycnt)) $={ }^{\mathrm{GD} 162}$.

curies (ycnt, isonum (ycnt)) $=9.862 \mathrm{E}-6 *$ tcurie

isonum (ycnt) $=$ isonum (ycnt) +1

nucnam (ycnt, isonum (ycnt)) = 'NB95'

curies (ycnt, isonum (ycnt)) $=1.053 \mathrm{E}-1 *$ tcurie

isonum (ycnt) $=$ isonum (ycnt) +1

nucnam (ycnt, isonum (ycnt)) = 'NB95M'

curies (ycnt, isonum (ycnt) ) $1.059 \mathrm{E}-1$ *tcurie

isonum (ycnt) $=$ isonum (ycnt) +1

nucram (ycnt, isonum (ycnt)) = 'PD107'

curies (ycnt, isonum (ycnt)) $=8.5 E-9 *$ tcurie

isonum (ycnt) $=$ isonum (ycnt) +1

nucnam (ycnt, isonum (ycnt)) $=$ 'PM147'

curies (ycnt, isonum (ycnt)) $=7.022 \mathrm{E}-2 *$ tcurie

isonum (ycnt) $=i$ sonum (ycnt) +1

nucnam (ycnt, isonum (ycnt)) = 'PR144

curies (ycnt, isonum (ycnt)) $=2.784 \mathrm{E}-1 *$ tcurie

isonum (ycnt) $=i \operatorname{sonum}(y c n t)+1$

nucnam (ycnt, isonum (ycnt)) $={ }^{\circ}$ RH106

curies (ycnt, isonum (ycnt)) $=4.051 \mathrm{E}-2 \star$ tcurie

isonum (ycnt) $=i \operatorname{sonum}(y c n t)+1$

nucnam (ycnt, isonum (ycnt)) $=$ RU106'

curies (ycnt, isonum (ycnt)) $=4.051 \mathrm{E}-2{ }^{\star}$ tcurie

isonum (ycnt) $=$ isonum (ycnt) +1

nucnam (ycnt, isonum (ycnt)) = SB125

curies (ycnt, isonum (ycnt)) $=7.985 \mathrm{E}-4{ }^{*}$ tcurie

isonum (ycnt) $=$ isonum (ycnt) +1

nucnam (ycnt, isonum (ycnt)) = 'SB126'

curies (ycnt, isonum (ycnt)) $=1.094 \mathrm{E}-7 *$ tcurie

isonum (ycnt) $=$ isonum (ycnt) +1

nucnam (ycnt, isonum (ycnt)) $={ }^{\prime} \mathrm{SB} 126 \mathrm{M}$ '

curies (ycnt, isonum (ycnt)) $=1.105 \mathrm{E}-7 *$ tcurie

isonum (ycnt) $=i$ sonum (ycnt) +1

nucnam (ycnt, i sonum (yCnt)) = 'SE79.

curies (ycnt, isonum (ycnt)) $=4.99 \mathrm{E}-8 *$ tcurie

isonum (ycnt) $=$ isonum (ycnt) +1

nucnam (ycnt, isonum (ycnt)) ='SM151'

PRE0 456

PRE0 457

PRE0 458

PRE0 459

PRE0 460

PRE0461

PRE0 462

PRE0 063

PRE0464

PRE0465

PRE0 466

PRE0467

PRE0468

PRE0469

PRE0470

PRE0471

PRE0472

PRE0473

PRE0 474

PRE0475

PRE0476

PRE0 477

PRE0478

PRE0479

PRE0480

PRE0481

PRE0482

PRE0483

PRE0484

PRE0485

PRE0486

PRE0487

PRE0488

PRE0489

PRE0490

PRE0491

PRE0 492

PRE0493

PRE0494

PRE0495

PRE0496

PRE0497

PRE0498

PRE0499

PRE0500

PRE0501

PRE0502

PRE0503

PRE0504

PRE0505

PRE0506

PRE0507

PRE0508

PRE0509

PRE0510

PRE0511

PRE0512

PRE0513

PRE0514

PRE0515

PRE0516

PRE0517

PRE0518

PRE0519

PRE0520

PRE0521

PRE0522

PRE0523

PRE0524

PRE0525

PRE0526

PRED527

PRE0528

PRE0529

PRE0530

PRE0531 
curies (ycnt, isonum (ycnt)) $=1.938 \mathrm{E}-4$ *tcurie

isonum (ycnt) $=$ isonum (ycnt) +1

nucnam (ycnt, isonum (ycnt)) = 'SN119M.

curies (ycnt, isonum (ycnt)) $=4.372 \mathrm{E}-5{ }^{\star}$ tcurie

isonum (ycnt) $=$ isonum (ycnt) +1

nucnam (ycnt, isonum (ycnt)) = ' $\operatorname{SN121M}$ '

curies (ycnt, isonum (ycnt)) $=3.942 \mathrm{E}-6 *$ tcurie

isonum (ycnt) $=$ isonum (ycnt) +1

nucnam (yCnt, isonum (ycnt)) = 'SN123M'

curies (ycnt, isonum $(y c n t))=1.847 E-5 *$ tcurie

isonum (ycnt) $=$ isonum (ycnt) +1

nucnam (ycnt, isonum (ycnt)) $={ }^{\prime}$ SN126'

curies (ycnt, isonum (ycnt) ) $=1.105 \mathrm{E}-7{ }^{\star}$ tcurie

isonum (ycnt) $=$ isonum (ycnt) +1

nucnam (ycnt, isonum (ycnt)) $=$ 'SR90'

curies (ycnt, isonum (ycnt)) $=1.881 \mathrm{E}-2 *$ tcurie

isonum (ycnt) $=$ isonum (ycnt) +1

nucnam (ycnt, isonum (ycnt)) = 'TB162.

curies (ycnt, isonum (ycnt)) $=9.862 \mathrm{E}-6 *$ tcurie

isonum (ycnt) $=$ isonum (ycnt) +1

$\operatorname{nucnam}(y c n t$, isonum $(y \subset n t))=1 \mathrm{TC} 99$.

curies (ycnt, isonum (ycnt)) $=2.867 \mathrm{E}-6 * t$ curie

isonum (ycnt) $=$ isonum (ycnt) +1

nucnam (ycnt, isonum (ycnt)) $=$ TE125M.

curies (ycnt, isonum (ycnt)) $=2.178 \mathrm{E}-4{ }^{*}$ tcurie

isonum (ycnt) $=i \operatorname{sonum}(y \circ n t)+1$

nucnam (ycnt, isonum (ycnt)) $={ }^{\prime} T E 127$.

curies (ycnt, isonum (ycnt)) $=1.354 \mathrm{E}-3 *$ tcurie

isonum (ycnt) $=$ isonum (ycnt) +1

nucnam (ycnt, isonum (ycnt)) = TE127M.

curies (ycnt, isonum (ycnt)) $=1.369 \mathrm{E}-3^{*}$ tcurie

isonum (ycnt) $=i \operatorname{sonum}(y \circ n t)+1$

nucnam (ycnt, isonum (ycnt)) $=\cdot Y 90$

curies (ycnt, isonum (ycnt)) $=1.881 \mathrm{E}-2 \star$ tcurie

isonum (ycnt) = isonum (ycnt) +1

nucnam (ycnt, isonum (ycnt)) = 'zR93'

curies (ycnt, isonum (ycnt)) $=6.455 \mathrm{E}-7{ }^{*}$ tcurie

isonum (ycnt) $=i \operatorname{sonum}(y \operatorname{cnt})+1$

nucnam (ycnt, isonum (ycnt)) = 'zR95'

curies (ycnt, isonum (ycnt)) $=4.988 \mathrm{E}-2 *$ tcurie

isonum (ycnt) $=$ isonum $(y<n t)+1$

ENDIF

GO TO 1020

GO TO 1020

C

c

8000 WRITE (6*)' INVENTORY DATA FOR FOR THE FOLLOWING YEARS: '

DO $15 i=0$,yent $-1,5$

WRITE $(6, *)(y 工(j), j=i+1, i+5)$

15 CONTINUE

5030

WRITE $(50,5030)$ outfil, $\operatorname{yr}(1), y r(n o y r)$, site, year

FORMAT (T5, A12, T24, I4,' THRU ', I4, T45, A15, 10X, A4)

WRITE $(50,5035)$ iu 234 , iu235, iu238

5035 FORMAT (t24, 'Enriched U Atom Fractions: U234= ',f5.2,' U235= ', $\mathrm{f5.2,} \mathrm{U} 238=\cdot \mathrm{f5} .2,1$

WRITE $(50,5000)$

WRITE $(50$, *

out $=15$

Do 60 cnt=1, nofile

C

C WRITE TO ORIGEN2 PC INPUT FILE

WRITE (OUT, 5040 )

5040 FORMAT $\left.(2(2 \mathrm{x}, \cdot-1 \cdot, /), 2 \mathrm{x}, \cdot-1)^{\prime}\right)$

WRITE (OUT, 5060 )

5060 FORMAT ( $2 x$, 'BAS', $4 x$, 'WIPP BASELINE INVENTORY DATA')

WRITE (OUT, 5065) title

5065 FORMAT ( $2 \mathrm{x}$, 'TIT', $4 \mathrm{x}, \mathrm{a} 80)$

WRITE (OUT, 5070) site

5070 FORMAT (2x, 'RDA', 4x, a15)

WRITE (OUT, 5075)

5075 FORMAT $(2 \mathrm{X}, \cdot \operatorname{LIP}, 4 \mathrm{x}, 3(\cdot 0 \cdot, 2 \mathrm{x}))$

WRITE (OUT, 5080)
PRE0533

PRE0534

PRE0535

PRE0536

PRE0537

PRE0538

PRE0539

PRE0540

PRE0541

PRE0542

PRE0543

PRE0544

PRE0545

PRE0546

PRE0547

PRE0548

PRE0 549

PRE0550

PRE0551

PRE0 552

PRE0553

PRE0554

PRE0555

PRE0556

PRE0 557

PRE0558

PRE0 559

PRE0560

PRE0561

PRE0562

PRE0563

PRE0 564

PRE0 565

PRE0566

PRE0567

PRE0 568

PRE0569

PRE0 0570

PRE0571

PRE0 572

PRE0573

PRE0574

PRE0575

PRE0576

PRE0577

PRE0578

PRE0579

PRE0580

PRE0581

PRE0582

PRE0583

PRE0 584

PRE0585

PRE0586

PRE0587

PRE0588

PRE0589

PRE0590

PRE0591

PRE0592

PRE0593

PRE0594

PRE0595

PRE0596

PRE0597

PRE0598

PRE0599

PRE0600

PRE0601

PRE0602

PRE0603

PRE0604

PRE0605

PRE0606

PRE0607 


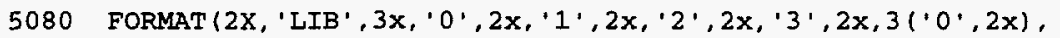
$+\quad 91,2 x, 3^{\prime}, 2 x, 0 \cdot, 2 x, 1^{\prime}, 2 x, 1^{\prime \prime}$

WRITE (OUT, 5085)

5085 FORMAT $\left(2 \mathrm{x},{ }^{\prime}\right.$ PHO', $\left.4 \mathrm{x}, \mathrm{x}^{\prime} 101^{\prime}, 2 \mathrm{x}, \cdot 102^{\prime}, 2 \mathrm{x}, \cdot 103^{\prime}, 2 \mathrm{x}, \cdot 10^{\prime}\right)$

c

C WRITE COMMANDS TO READ INPUT IN ORIGEN2 INPUT FILE

C

IF (nofile.EQ.1) THEN

$y \max =y c n t$

init $=1$

ELSEIF (cnt.NE. nofile) THEN

init $=y \max +1$

$y \max =$ init +14

ELSEIF (cnt. EQ. nofile) THEN

init $=y \max +1$

$y \max =y \operatorname{cnt}$

C

ENDIF

Do $20 i=$ init, $y \max$

WRITE (OUT, 5090) yr(i)

5090 FORMAT ( $2 x, \cdot R D A \cdot, 4 x$, 'INITIAL VALUES FOR: $\cdot, i 4)$ $j=-(1-($ cnt -1$) * 15)$

WRITE (OUT, 5095) j

5095 FORMAT $(2 \mathrm{x}$, 'INP', 4x, i4, 2x, ' 1 ' , 2x, 2('-1', 2x), 2('1', 2x $)$

20 CONTINUE

C WRITE ORIGEN2 COMMANDS TO DECAY ALL YEARS IN DATABASE

C FILE OUT TO YEAR GIVEN BY USER, INSTRUCT ORIGEN2 TO OUTPUT

C EACH YEAR AS CALCULATION IS PERFORMED IF USER SETS IOUT $=1$

C AND ADD TO A VECTOR TO HOLD ALL OF YEAR GIVEN BY USER DECAY DATA

C

WRITE (OUT, 5100) fyr, input (1:8)

5100 FORMAT ( $2 x$, 'BAS', 4x, 'DECAY INITIAL INVENTORIES TO: ' i4, ':', AB)

C

Do $25 i=$ init, ymax

$j=-(i-($ cnt -1$) * 15)$

$\operatorname{decay}(i)=\operatorname{dble}(f y r-y r(i))$

5105

WRITE (OUT, 5105) yr(i), decay (i)

$\&$

FORMAT ( $2 x$, 'RDA', $4 x$, ' DECAY YEAR ', i4, $2 x$, 'TO',

ivec $=\operatorname{abs}(j)$

WRITE(OUT, 5110) decay $(i), j$, ivec

5110

FORMAT ( $2 x$, 'DEC' $\left., 4 x, f 7.2,2 x, 13,2 x, 13,2 x, \cdot 5^{\prime}, 2 x, ' 1{ }^{\prime}\right)$

WRITE (OUT, 5115) yr(i), EYI

5115

FORMAT ( $2 x$, 'RDA', $4 x$, 'OUTPUT ', 14, ' DECAY TO ', i4, ' DATA ')

WRITE (OUT, 5120) ivec

5120

FORMAT ( $2 \mathrm{x}$, 'MOV', $\left.4 \mathrm{x}, 13,2 \mathrm{x}, \mathrm{\prime}^{\prime}, 2 \mathrm{x}, \mathrm{\prime}^{\prime}, 2 \mathrm{x},{ }^{\prime}\right)^{\prime}$ )

WRITE (OUT, 5125) $j$

FORMAT ( $2 \mathrm{x}$, 'MOV', $4 \mathrm{x}, 13,2 \mathrm{x}, 11 \cdot, 2 \mathrm{x}, \mathrm{\prime}^{\prime}, 2 \mathrm{x}, \mathrm{\prime}^{\prime}$ ')

5125

WRITE (OUT, 5130) YI(i)

5130

FORMAT ( $2 \mathrm{X}$, 'HED', $4 \mathrm{x}, 11$ ', 2x, i4)

WRITE (OUT, 5135) fYY

5135

5140

FORMAT ( $\left.\left.2 \mathrm{X},{ }^{\prime} \mathrm{HED}\right)^{\prime}, 4 \mathrm{x},{ }^{\prime} 2 \cdot, 2 \mathrm{x}, \mathrm{i} 4\right)$

WRITE (OUT, 5140)

FORMAT ( $2 \mathrm{x}$, 'RDA', $4 \mathrm{x}$, 'PRINT NUCLIDE QUANTITIES IN CURIES')

5145

WRITE (OUT, 5145)

5150

WRITE (OUT, 5150 )

FORMAT $(2 x$, , OPTF

5155

WRITE (OUT', 5155)

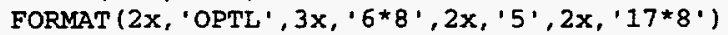

WRITE (OUT, 5160)

5160

作

WRITE (OUT, 5165)

5165

C

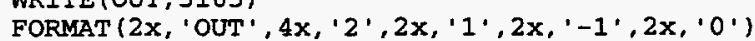

25 CONTINUE

c

IF (cnt.EQ.nofile) THEN

WRITE (OUT', 5170)

5170

FORMAT ( $2 \mathrm{x}$, 'END')

ELSEIF (cnt.NE. nofile) THEN

WRITE (OUT, 5175)

5175

ENDIF

FORMAT (2x, 'STP', $4 x, 1$ ')

PRE0608

PRE0609

PRE0610

PRE0611

PRE0612

PRE0 613

PRE0614

PRE0615

PRE0616

PRE0 617

PRE0618

PRE0619

PRE0620

PRE0621

PRE0622

PRE0623

PRE062 4

PRE0625

PRE0626

PRE0627

PRE0628

PRE0629

PRE0630

PRE0631

PRE0632

PRE0633

PRE0634

PRE0635

PRE0636

PRE0637

PRE0638

PRE0639

PRE0640

PRE0641

PRE0642

PRE0643

PRE0644

PRE0645

PRE0646

PRE0647

PRE0648

PRE0649

PRE0650

PRE0 651

PRE0 652

PRE0653

PRE0654

PRE0655

PRE0656

PRE0657

PRE0658

PRE0659

PRE0 660

PRE0661

PRE0662

PRE0663

PRE0664

PRE0665

PRE0666

PRE0667

PRE0668

PRE0669

PRE0670

PRE0671

PRE0 672

PRE0673

PRE0674

PRE0 675

PRE0676

PRE0677

PRE0678

PRE0679

PRE0680

PRE0681

PRE0682

PRE0683 
C

C

WRITE INITIAL NUCLIDE INVENTORIES TO ORIGEN2 INPUT FILE

PRE0684

Do $55 i=$ init, $y \max$

num $=i$ sonum $(i)$

lib1 $=0$

$1 \mathrm{ib} 2=0$

$\operatorname{lib3}=0$

lent $=0$

C CONVERT INITIAL NUCLIDE CONCENTRATIONS FROM CURIES TO GRAMS AND

C ASSIGN ORIGEN2 SPECIFIC NUCLIDE IDENTIFIER

C

DO $30 j=1$, num

CALL CONV (nucnam $(i, j), \operatorname{curies}(i, j), \operatorname{nucid}(i, j)$,

$30 \&$ CONTINUE

$\operatorname{grams}(i, j), \operatorname{lib}(i, j))$

DO $35 \mathrm{k}=1$, num

$\operatorname{IF}(1 \mathrm{ib}(i, k)$.EQ.1) THEN

$1 i b 1=1 i b 1+1$

$\operatorname{var} 1(1 \mathrm{ib} 1)=\mathrm{k}$

ELSEIF ( $1 i b(i, k)$.EQ.2) THEN

$1 i b 2=1 i b 2+1$

$\operatorname{var} 2(1 \mathrm{ib} 2)=\mathrm{k}$

ELSEIF $(1 i b(i, k) . E Q .3)$ THEN

$1 i b 3=1 i b 3+1$

ENDIF

$\operatorname{var} 3(1 \mathrm{ib} 3)=k$

CONTINUE

C

C FOR PRESENT YEAR, FROM LIBRARY 1 - ACTIVATION PRODUCTS

IF (Iib1.LE. 3) THEN

num $=1 \mathrm{ib} 1$

IF (lib1.EQ.1) THEN

WRITE (OUT, 5185) (nucid(i, $\operatorname{var} 1(L)), \operatorname{grams}(i, \operatorname{var} 1(L)), L=1,1 i b 1)$

5185

FORMAT ( $2 x, \cdot 1{ }^{\prime}, 5 x, A 7,2 x$, e $\left.10.4,2 x, 0^{\prime}, 2 x, 0^{\prime}\right)$ ELSEIF ( lib1.EQ.2) THEN

WRITE (OUT, 5190) (nucid(i, $\operatorname{var} 1(L)), \operatorname{grams}(i, \operatorname{var} 1(L)), L=1,2$ )

5190 FORMAT $\left(2 x, \cdot 11,5 x, 2(a 7,2 x\right.$, e10.4, $\left.2 x), 0^{\prime}, 2 x, 0^{\prime}\right)$ ELSEIF ( 1 ibl.EQ.3) THEN

WRITE (OUT, 5195) (nucid (i, $\operatorname{var} 1(L)), \operatorname{grams}(i, \operatorname{var} 1(L)), L=1,3$ )

5195 FORMAT $\left(2 \mathrm{x}, 11,5 \mathrm{x}, 3(\mathrm{a} 7,2 \mathrm{x}, \mathrm{e} 10.4,2 \mathrm{x}), 0^{\prime}, 2 \mathrm{x}, 0^{\prime}\right)$ ENDIF

ELSEIF ( IIB 1.GT.3) THEN

Idiv1 $=$ int $(1 \mathrm{ib} 1 / 3)$

ldiv2 = lib1 - 3*1div1

$\mathrm{m}=0$

IF (Idiv2.EO.0) THEN

$m=m+3$

IF (m.LE. Iib1) THEN

$$
\mathrm{n}=\mathrm{m}-2
$$

WRITE (OUT, 5200) (nucid(i, $\operatorname{var} 1(\mathrm{~L})$ ), $\operatorname{grams}(i, \operatorname{var} 1(L)), \mathrm{L}=\mathrm{n}, \mathrm{m}$ )

5200

FORMAT $(2 x, ' 1 \cdot, 5 x, 3(a), 2 x, e 10.4,2 x), \cdot 0 \cdot, 2 x, \cdot 0 \cdot)$ Go TO 1025

ENDIF

ELSEIF (1div2.GT . 0) THEN IF (ldiv1.EO.1) THEN

WRITE (OUT, 5200) (nucid( $i, \operatorname{var} 1(L)), \operatorname{grams}(i, \operatorname{var}(L)), L=1,3$ ) IF(ldiv2. EQ.1) THE

WRITE (OUT, 5185) (nucid ( $i, \operatorname{var} 1(L)), \operatorname{grams}(i, \operatorname{var} 1(L)), L=4,1 i b 1)$ ELSEIF (1div2.EQ.2) THEN

WRITE (OUT, 5190) (nucid( $i, \operatorname{var} 1(L)), \operatorname{grams}(i, \operatorname{var} 1(L)), L=4,1 i b 1$ ) ENDIF ELSE

DO $40 \mathrm{k}=1,1 \mathrm{div} 1$

$m=m+3$

lent $=1$ cnt +1

IF (lcnt.LE. ldiv1) THEN

$\mathrm{n}=\mathrm{m}-2$

WRITE (OUT, 5200$)($ nucid $(i, \operatorname{var} 1(I)), \operatorname{grams}(i, \operatorname{var} 1(L)), L=n, m$ ) GO TO 1030

ELSEIF ( lcnt.GT. Idiv1) THEN

$\mathrm{m}=\mathrm{m}-3$

ENDIF

PRE0685

PRE0686

PRE0687

PRE0688

PRE0689

PRE0690

PRE0691

PRE0692

PRE0693

PRE0694

PRE0695

PRE0696

PRE0697

PRE0698

PRE0699

PRE0700

PRE0701

PRE0702

PRE0703

PRE0704

PRE0705

PRE0706

PRE0707

PRE0708

PRE0709

PRE0710

PRE0711

PRE0712

PRE0713

PRE0714

PRE0715

PRE0716

PRE0717

PRE0718

PRE0719

PRE0720

PRE0721

PRE0722

PRE0723

PRE0724

PRE0725

PRE0726

PRE0727

PRE0728

PRE0729

PRE0730

PRE0731

PRE0732

PRE0733

PRE0734

PRE0735

PRE0736

PRE0737

PRE0738

PRE0739

PRE0740

PRE0741

PRE0742

PRE0743

PRE0744

PRE0745

PRE0746

PRE0747

PRE0748

PRE0749

PRE0750

PRE0751

PRE0752

PRE0753

PRE0754

PRE0755

PRE0756

PRE0757

PRE0758

PRE0759 
WRITE (OUT, 5185) (nucid(i, var1 (L)), grams $(i, \operatorname{var} 1(L)), L=m, 1 i b 1)$ ELSEIF (ldiv2. EQ.2) THEN

WRITE (OUT, 5190) (nucid( $i, \operatorname{var} 1(L)), \operatorname{grams}(i, \operatorname{var} 1(L)), L=m, 1 i b 1)$ ENDIF ENDIF ENDIF

C FOR PRESENT YEAR, FROM LIBRARY 2 - ACTINIDES

lent $=0$

IF (Iib2.LE.3) THEN num $=1 \mathrm{ib} 2$ IF (I Ib2.EQ.1) THEN

WRITE (OUT, 5205) (nucid(i, $\operatorname{var} 2(L)), \operatorname{grams}(i, \operatorname{var} 2(L)), L=1,1 i b 2$ )

FORMAT $\left(2 x,{ }^{\prime} 2{ }^{\prime}, 5 x, A 7,2 x\right.$, e10.4, $\left.2 x, 0^{\prime}, 2 x, 0^{\prime}\right)$ ELSEIF ( 1 ib2.EQ.2) THEN

WRITE (OUT, 5210) (nucid $(i, \operatorname{var} 2(L)), \operatorname{grams}(i, \operatorname{var} 2(L)), L=1,2$ )

5210 FORMAT $\left(2 \mathrm{x},{ }^{\prime} 2, .5 \mathrm{x}, 2(\mathrm{a} 7,2 \mathrm{x}, \mathrm{e} 10.4,2 \mathrm{x}), 0^{\prime}, 2 \mathrm{x}, \mathrm{\prime}^{\prime}\right)$ ELSEIF ( 1 ib2 . EQ.3) THEN

WRITE (OUT, 5215) (nucid $(i, \operatorname{var} 2(L)), \operatorname{grams}(i, \operatorname{var}(L)), L=1,3$ )

5215

FORMAT $(2 x, \cdot 2 \cdot, 5 x, 3(a 7,2 x, e 10.4,2 x), \cdot 0 \cdot, 2 x, \cdot 0 \cdot)$ ENDIF

ELSEIF (I Ib2.GT.3) THEN

laiv1 $=\operatorname{int}(1 \mathrm{ib} 2 / 3)$

ldiv2 = lib2 $-3 * 1$ div1

$\mathrm{m}=0$

IF (laiv2.EQ.0) THEN

1035

$\mathrm{m}=\mathrm{m}+3$

IF (m. LE. I Ib2) THEN $\mathrm{n}=\mathrm{m}-2$

WRITE (OUT, 5220) (nucid(i, $\operatorname{var2}(L)), \operatorname{grams}(i, \operatorname{var}(L)), L=n, m$ )

FORMAT $\left(2 \mathrm{x}, \mathrm{\prime}^{\prime}, 5 \mathrm{x}, 3(\mathrm{a} 7,2 \mathrm{x}, \mathrm{e} 10,4,2 \mathrm{x}), 0^{\prime}, 2 \mathrm{x}, 0^{\prime}\right)$ GO TO 1035

ENDIF

ELSEIF (1div2.GT.0) THEN

IF (Idiv1.EQ.1) THEN

WRITE (OUT, 5220$)(\operatorname{mucid}(i, \operatorname{var} 2(L)), \operatorname{grans}(i, \operatorname{var} 2(L)), L=1,3$ ) IF (ldiv2.EQ.1) THEN

WRITE (OUT, 5205) (nucid (i, var2 $(L)), \operatorname{grams}(i, \operatorname{var} 2(L)), L=4,1 i b 2)$ ELSEIF (laiv2. EQ.2) THEN

WRITE (OUT, 5210) (nucid( $i, \operatorname{var} 2(L)), \operatorname{grams}(i, \operatorname{var} 2(L)), L=4,1 i b 2)$ ENDIF

ELSE

DO $45 \mathrm{k}=1$, Idiv1

1040

$\mathrm{m}=\mathrm{m}+3$

lent $=1$ cnt +1

IF (lCnt. LE. Idiv1) THEN

$\mathrm{n}=\mathrm{m}-2$

WRITE (OUT, 5220 ) (nucid(i, $\operatorname{var2}(L)), \operatorname{grams}(i, \operatorname{var}(L)), L=n, m$ ) GO TO 1040

ELSEIF (lcnt. GT . ldiv1) THEN

$m=m-3$

ENDIF

45

CONTINUE

$\mathrm{m}=\mathrm{m}+1$

IF (ldiv2.EQ.1) THEN

WRITE (OUT, 5205) (nucia ( $i, \operatorname{var} 2(L)), \operatorname{grams}(i, \operatorname{var2}(L)), L=m, 1 i b 2)$ ELSEIF (ldiv2.EQ.2) THEN

WRITE (OUT, 5210) (nucid ( $i, \operatorname{var} 2(L)), \operatorname{grams}(i, \operatorname{var} 2(L)), L=m, 1 i b 2$ ) ENDIF

$$
\text { ENDI }
$$

ENDIF

C FOR PRESENT YEAR, FROM LIBRARY 3 - FISSION PRODUCTS

$$
\begin{aligned}
& \text { lcnt }=0 \\
& \text { IF (Iib3. LE. } 3 \text { ) THEN } \\
& \text { num }=\text { lib3 }
\end{aligned}
$$

IF ( 1 ib3.EQ.1) THEN

PRE0760

PRE0761

PRE0762

PRE0763

PRE0764

PRE0765

PRE0766

PRE0767

PRE0768

PRE0769

PRE0770

PRE0771

PRE0772

PRE0773

PRE0774

PRE0775

PRE0776

PRE0777

PRE0778

PRE0779

PRE0780

PRE0781

PRE0782

PRE0783

PRE0784

PRE0785

PRE0786

PRE0787

PRE0788

PRE0789

PRE0790

PRE0791

PRE0792

PRE 0793

PRE0 794

PRE0795

PRE0796

PRE0797

PRE0798

PRE0799

PREO 800

PREO 801

PRE0802

PRE0803

PRE0 804

PRE0805

PRE0806

PRE0 807

PREO 808

PRE0809

PRE0810

PRE0811

PRE0812

PRE0813

PRE0814

PRE0815

PRE0816

PRE0817

PRE0 818

PRE0819

PRE0820

PRE0821

PRE0822

PRE0823

PRE0 824

PRE0825

PRE0826

PRE0 827

PRE0828

PRE0829

PRE0830

PRE0831

PRE0832

PRE0833

PRE0834

PRE0835 
ELSEIF (1ib3.EO. 2) THEN

WRITE (OUT, 5230) (nucid(i, $\operatorname{var} 3(L)), \operatorname{grams}(i, \operatorname{var} 3(L)), L=1,2$ )

FORMAT $\left(2 x,{ }^{\prime} 3,5 x, 2(a), 2 x\right.$, e10.4, $\left.\left.2 x\right), 0^{\prime}, 2 x, 0^{\prime}\right)$ ELSEIF (1ib3.EQ.3) THEN

WRITE (OUT, 5235) (nucid( $i, \operatorname{var} 3(L)), \operatorname{grams}(i, \operatorname{var} 3(L)), L=1,3$ )

FORMAT $\left(2 x, 13^{\prime}, 5 x, 3(27,2 x, e 10,4,2 x), 0^{\prime}, 2 x, 0^{\prime}\right)$ ENDIF

ELSEIF ( 1 ib3.GT . 3) THEN

ldiv1 $=\operatorname{int}(1 \mathrm{ib} 3 / 3)$

ldiv2 = lib3 - 3*ldiv1

$m=0$

IF (Idiv2.EQ.0) THEN

1045

$\mathrm{m}=\mathrm{m}+3$

IF (M.LE.IIb3) THEN

$\mathrm{n}=\mathrm{m}-2$

WRITE (OUT, 5240) (nucid ( $i, \operatorname{var} 3(L)), \operatorname{grams}(i, \operatorname{var} 3(L)), I=n, m$ )

5240

FORMAT $\left(2 x, 3^{\prime}, 5 \mathrm{x}, 3(\mathrm{a} 7,2 \mathrm{x}, \mathrm{e} 10.4,2 \mathrm{x}), 0^{\prime}, 2 \mathrm{x}, \mathrm{\prime}^{\prime} 0^{\prime}\right)$

GO TO 1045

FIDIF

ELSEIF (Idiv2 . GT . 0) THEN IF (ldivi.EQ.1)THEN

WRITE (OUT, 5240) (nucid (i, var3 (L)), grams (i, var3(L)), $I=1,3$ ) IF (ldiv2.EQ.1) THEN

WRITE (OUT, 5225) (nucid ( $i, \operatorname{var} 3(L)), \operatorname{grams}(i, \operatorname{var} 3(L)), L=4,1 i b 3$ ) ELSEIF (1div2.EO.2) THEN

WRITE (OUT, 5230) (nucid( $i, \operatorname{var} 3(L)), \operatorname{grams}(i, \operatorname{var} 3(L)), L=4,1 i b 3$ ) ENDIF

ELSE

1050

DO $50 \mathrm{k}=1$, Idiv1

$m=m+3$

lcnt $=$ lcnt +1

IF (Icnt.LE. Iaiv1) THEN

$n=m-2$

WRITE (OUT, 5240$)(\operatorname{nucid}(i, \operatorname{var} 3(L)), \operatorname{grams}(i, \operatorname{var} 3(L)), L=n, m)$ GO TO 1050

ELSEIF (lent.GT. Idiv1) THEN

$m=m-3$

ENDIF

50

CONTINUE

$m=m+1$

IF (Idiv2.EQ.1) THEN

WRITE (OUT, 5225) (nucid(i, $\operatorname{var} 3(L)), \operatorname{grams}(i, \operatorname{var} 3(L)), L=m, 1 i b 3$ ) ELSEIF (ldiv2.EQ.2) THEN

WRITE (OUT, 5230) (nucid(i, var3 (L)), grams (i, $\operatorname{var} 3(L)), L=m, 1 i b 3$ ) ENDI

ENDIF

WRITE (OUT, 5245)

5245 FORMAT $(2 \times, \cdot 0 \cdot)$

55 CONTINUE

C

IF (NOFILE.EQ.1)GO TO 1055

60 CONTINUE

C GIVE USER OPTION TO RUN PROGRAM AGAIN

1055 WRITE $(6,5000)$

$\operatorname{WRITE}(6$, *

WRITE $(6, *)$ " WOULD YOU LIKE TO RUN PROGRAM AGAIN TO GENERATE

WRITE $(6, *)$ - MORE ORIGEN2 INPUT FILES? 1-YES 2-NO '

WRITE $(6, *)$

$\operatorname{WRITE}(6,5000)$

READ*, irun

IF(irun.EQ.1)GO TO 1000

9999 STOP

FND

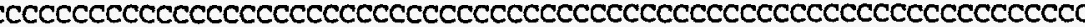
SUBROUTINE TYPE (itype, waste)

CCCCCCCCCCCCCCCCCCCCCCCCCCCCCCCCCCCCCCCCCCCCCCCCCCCCCCCCCCCCCCCCCCCCCCCC PRE0910 INTEGER i tYpe

PRE0836

PRE0837

PRE0 838

PRE0839

PRE0840

PRE0841

PRE0842

PRE0843

PRE0844

PRE0845

PRE0846

PRE0847

PRE0 848

PRE0849

PRE0850

PRE0851

PRE0852

PRE0853

PRE0854

PRE0855

PRE0856

PRE0 857

PRE0858

PRE0859

PRE0860

PRE0861

PRE0862

PRE0863

PRE0864

PRE0865

PRE0866

PRE0867

PRE0 868

PRE0869

PRE0870

PRE0871

PRE0872

PRE0873

PRE0874

PRE0875

PRE0876

PRE0877

PRE0878

PRE0879

PRE0880

PRE0881

PRE0882

PRE0883

PRE0884

PRE0885

PRE 0886

PRE0887

PRE0888

PRE0889

PRE0890

PRE0891

PRE0892

PRE0893

PRE0894

PRE0895

PRE0896

PRE0897

PRE0898

PRE0899

PRE0900

PRE0901

PRE0902

PRE0903

PRE0904

PRE0905

PRE0906

PRE0908

PRE0911 
c

CHARACTER waste* 2

PREO912

WRITE $(6, *)$ ' PLEASE CHOOSE WASTE CATEGORY:

WRITE $(6, *) \cdot 1$ - CH TRU, 2 - RH TRU, OR 3 - BURIED TRU • $\operatorname{WRITE}(6, *)$

READ*, itype

C

IF (itype.EQ.1) THEN

waste $=$ ' $\mathrm{CH}$ '

ELSEIF (itype. EQ.2) THEN waste $=$ ' $\mathrm{RH}$ '

ELSEIF (ITYPE.EQ.3) THEN waste $=$ ' $\mathrm{BU}$ '

C ENDIF

RETURN

END

PRE0913

PREO914

PREO915

PRE0916

PRE0917

PRE0918

PRE0919

PRE0920

PRE0921

PRE0922

PRE0923

PRE0924

PRE0925

PRE0926

PRE0927

PRE0928

CCCCCCCCCCCCCCCCCCCCCCCCCCCCCCCCCCCCCCCCCCCCCCCCCCCCCCCCCCCCCCCCCCCCCCCC PRE0929 SUBROUTINE INNAME (site, itype, input)

PRE0930

CCCCCCCCCCCCCCCCCCCCCCCCCCCCCCCCCCCCCCCCCCCCCCCCCCCCCCCCCCCCCCCCCCCCCCCC PRE0931

CHARACTER site 7 , input* 12

INTEGER itype

C

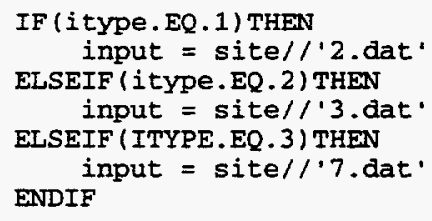

C

WRITE $(6, \star)$ ' PreORG INPUT DATA FILENAME: $\because$ input

C RETURN

END

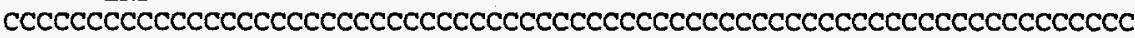
SUBROUTINE NUMYRS (input, noyr, nofile)

CCCCCCCCCCCCCCCCCCCCCCCCCCCCCCCCCCCCCCCCCCCCCCCCCCCCCCCCCCCCCCCCCCCC

C THIS SUBROUTINE WILI COUNT THE NUMBER OF YEARS IN A DATA FILE

C TO DETERMINE HOW MANY ORIGEN2 INPUT FILES MUST BE USED TO DECAY

C ALI THE INVENTORIES OUT AS DESIRED. THIS IS DUE TO LIMITATIONS

C IN THE ORIGEN2 CODE.

CCCCCCCCCCCCCCCCCCCCCCCCCCCCCCCCCCCCCCCCCCCCCCCCCCCCCCCCCCCCCCCCCCC

IMPLICIT real $(a-h, 0-z)$, integer $(i-n)$

INTEGER noyr, nofile

CHARACTER input* 12 , line*133

C

C

OPEN (UNIT $=10$, FILE $=$ input , STATUS $=$ ' OLD' $)$

noyr $=0$

nofile $=0$

$1000 \operatorname{READ}(10,5000, \operatorname{END}=1100)$ line

5000 FORMAT (A133)

C

IF (1ine $(1: 2)$. EQ.'19!) THEN

ENDIF noyr $=$ noyr +1

C

GO TO 1000

1100 IF (nOYY.LE.15) THEN

nofile $=1$

ELSEIF (nOYY. GT . 15) THEN

ldivl $=$ int $($ noyr $/ 15)$

ldiv2 $=$ noyr - ldiv1 15

IF (ldiv2. EO.0) nofile = ldiv1

ENDIF

IF (ldiv2.gt.0) nofile=ldiv1+1

c

CLOSE (UNIT $=10$ )

RETURN

END

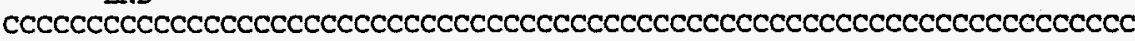
SUBROUTINE OUTNAM (year, input, outfil)

CCCCCCCCCCCCCCCCCCCCCCCCCCCCCCCCCCCCCCCCCCCCCCCCCCCCCCCCCCCCCCCCCCCC

$C$ THIS SUBROUTINE WILL COUNT THE NUMBER OF YEARS IN A DATA FILE

PRE0932

PRE0933

PRE0934

PRE0935

PRE0936

PRE0937

PRE0938

PRE0939

PRE0940

PRE0941

PREO942

PRE0943

PREO944

PRE0945

PRE0946

PRE0947

PRE0948

PRE0949

PRE0950

PRE0951

PRE0952

PRE0953

PRE0954

PRE0955

PRE0956

PRE0957

PRE0958

PRE0959

PRE0960

PRE0961

PRE0962

PRE0963

PRE0964

PRE0965

PRE0966

PRE0967

PRE0968

PRE0969

PRE0970

PRE0971

PRE0 072

PRE0973

PRE0974

PRE0975

PRE0976

PRE0977

PRE0978

PRE0979

PRE0980

PRE0981

PRE0982

PRE0983

PRE0984

PRE0985

PRE0986

PRE0987 
C TO DETERMINE HOW MANY ORIGEN2 INPUT FILES MUST BE USED TO DECAY C ALL THE INVENTORIES OUT AS DESIRED. THIS IS DUE TO LIMITATIONS C IN THE ORIGEN2 CODE.

CCCCCCCCCCCCCCCCCCCCCCCCCCCCCCCCCCCCCCCCCCCCCCCCCCCCCCCCCCCCCCCCCCCC IMPLICIT real $(a-h, o-z)$, integer $(i-n)$ CHARACTER input*12, outfil*12, type*2, year*4

C

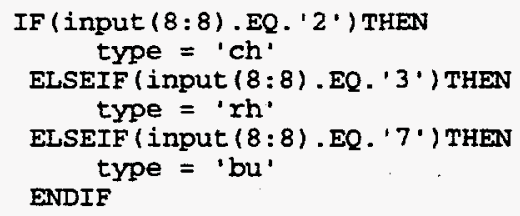

c

outfil = input $(1: 4) / /$ type $/ /$ year $(3: 4) / / 1.45$ WRITE $(6, *)$ ' PreORG OUTPUT FILENAME: $'$, outfil

c RETURN END WRITE $(6, *) \cdot$ PLEASE ENTER SITE NAME:

PRE1012

PRE1013

PRE1014

READ 2, name

2 FORMAT (A12)

WRITE $(6, *)$ ' PREORG ACCEPTS FOLLOWING SITE NAME ON ALI FILES: ‘

PRE1015

PRE1016

PRE1017

site $=$ name $(1: 7)$

WRITE $\left(6,{ }^{*}\right)$ ' SITE NAME: ', site

C

$\mathrm{ch}=$ site//'2.dat'

$\mathrm{rh}=$ site//'3.dat

bu $=$ site//'7.dat

$c$

WRITE $(6, *)$ ' TO RUN SUCESSFULLY THE FOLLOWING WASTE TYPES MUST ' PRE1018

PRE1019

PRE1020

PRE1021

PRE1022

PRE1023

PRE1024

PRE1025

WRITE $(6, *)$ ' HAVE THE FOLTOWING NAMES:

$\operatorname{WRITE}(6, *)$

$\operatorname{WRITE}(6, *)$, CH WASTE

$\operatorname{WRITE}(6, *)$

WRITE $(6, \bullet)$, RH WASTE ', rh

$\operatorname{WRITE}(6, *)$

WRITE $(6, \bullet)$, BURIED WASTE 1 , bU

C

WRITE $(6, \star)$

PRE1026

PRE1027

PRE1028

PRE1029

PRE1030

PRE1031

PRE1032

PRE1033

PRE1034

PRE1035

RETURN

PRE1036

END

PRE1037

SUBROUTINE CONV (name, activ, namy, activy, nuclib)

C THIS SUBROUTINE WAS WRITTEN BY HOLIY TRELLUE, $6 / 8 / 95$

C MODIFIED BY J.L. POWELL 6/29/95 WITH ORIGEN2 DECAY LIBRARY HALF LIVES

PRE1038

PRE1039

PRE1040

PRE1041

PRE1042

PRE1043

CHARACTER name* 6 , namy ${ }^{*}$

INTEGER nUclib

PRE1044

PRE1045

C ALI VALUES FOR ATOMTC MASS MUMBERS AND HALFI TVES TN THIS FILE WERE TAK PRE1046

C FROM THE CHART OF THE NUCLIDES.

$$
\text { IF (name }(1: 5) . E Q \cdot{ }^{\prime} \text { AC227') THEN }
$$

C ATOMIC WEIGHT VALUES(M) WERE CALCULATED BY USING THE MASS EXCESS VALUE PREI051

C FOR EACH ISOTOPE. MASS EXCESS VALUES WERE TAKEN FROM "NUCLEAR WALLET

C CARDS" BY JAGDISH TULI. JANUARY 1985.

$$
\mathrm{m}=227.0+25.849 / 931.5016
$$

C FOR CONFORMITY, THE HALF IIVES ARE CONVERTED TO SECONDS FOR

C EVERY ISOTOPE. AC227'S HALF IIFE IS 21.77 YEARS. ALI VALUES FOR

C HALFLIVES IN THIS PROGRAM COME FROM THE ORIGEN2 DECAY LIBRARY

$$
\begin{aligned}
& \text { Thalf }=6.871 \mathrm{e} 08 \\
& \text { nuclib }=2 \\
& \text { namy }(1: 6)=\cdot 892270 .
\end{aligned}
$$

PRE1052

PRE1053

PRE1054

PRE1055

PRE1056

PRE1057

PRE1058

PRE1059

PRE1060

PRE1061

PRE1062

PRE1063 
ELSEIF (name (1:5).EQ.'AG110.) THEN

PRE1064

if (name $(6: 6) . E Q$. ' $M$ ') THEN

$\mathrm{m}=110.0-87.341 / 931.5016$

Thalf $=2.159 \mathrm{E07}$

namy $(1: 6)=\cdot 471101$ '

nuclib=3

ELSE

$m=110.0-87.459 / 931.5016$

Thalf $=2.460 E 01$

namy $(1: 6)=\cdot 471100$.

nuclib $=3$

ENDIF

ELSEIF (name (1:5).EQ. 'AM240') THEN

$\mathrm{m}=240.0+51.498 / 931.5016$

Thalf $=1.829 \mathrm{e} 05$

namy $(1: 6)=' 952400^{\prime}$

nuclib $=2$

ELSEIF (name (1:5) .EQ. 'AM241') THEN

$\mathrm{m}=241.0+52.931 / 931.5016$

Thalf $=1.364 \mathrm{e} 10$

$\operatorname{namy}(1: 6)=' 952410^{\prime}$

nucli $\mathrm{b}=2$

ELSEIF (name (1:5).EQ.'AM242') THEN $\mathrm{m}=242.0+55.463 / 931.5016$

Thalf $=5.767 \mathrm{e} 04$

namy $(1: 6)=' 952420^{\circ}$

nuclib $=2$

ELSEIF (name (1:5) .EQ. 'AM243') THEN

$m=243.0+57.169 / 931.5016$

Thalf $=2.329 \mathrm{e} 11$

namy $(1: 6)=\cdot 952430^{\prime}$

nuclib=2

ELSEIF (name (1:5). EQ.'AM244') THEN $\mathrm{m}=244.0+59.877 / 931.5016$

Thalf $=3.636 \mathrm{e} 04$

namy $(1: 6)=.952440^{\circ}$

nuclib $=2$

ELSEIF (name (1:6).EQ. 'BA137M') THEN $\mathrm{m}=137.0-87.070 / 931.5016$

Thal $f=1.531 \mathrm{e} 02$

namy $(1: 6)=' 561371$

nucl $i b=1$

ELSEIF (name (1:5).EQ.'BI214') THEN

$\mathrm{m}=214.0-1.218 / 931.5016$

Thal $f=1.194 \mathrm{e} 03$

namy $(1: 6)=\cdot 832140$ '

nuclib $=2$

ELSEIF (name (1:5).EQ.'BK249') THEN $m=249.0+69.843 / 931.5016$

Thalf $=2.765 \mathrm{e} 07$

$\operatorname{namy}(1: 6)=1972490^{\circ}$ nuclib=2

ELSEIF (name(1:3).EQ.'C14') THEN

$m=14.0+3.02 / 931.5016$

Thalf $=1.808 \mathrm{e} 11$

namy $(1: 6)=' 060140$

nucl $i b=1$

ELSEIF (name (1:5) .EO.'CDI09') THEN $m=109.0-88.507 / 931.5016$

Thalf $=4.009 \mathrm{e} 07$

namy $(1: 6)=\cdot 481090$

nuclib=1

ELSEIF (name $(1: 6) . E Q . ' C D 113 M '$ ) THEN

$m=113.0-88.786 / 931.5016$

Thalf $=4.604 \mathrm{E} 08$

namy $(1: 6)=' 481131$.

nuclib=3

ELSEIF (name (1:5).EQ.'CE144') THEN

$m=144.0-80.441 / 931.5016$

Thalf $=2.456 \mathrm{e} 07$

namy $(1: 6)=' 581440$ '

nuclib=1

ELSEIF (name (1:5) EO. 'CF249') THEIN

$m=249.0+69.717 / 931.5016$

Thalf $=1.106 \mathrm{e} 10$

namy $(1: 6)=' 982490$ '

PRE1065

PRE1066

PRE1067

PRE1068

PRE1069

PRE1070

PRE1071

PRE1072

PRE1073

PRE1074

PRE1075

PRE1076

PRE1077

PRE1078

PRE1079

PRE1080

PRE1081

PRE1082

PRE1083

PRE1084

PRE1085

PRE1086

PRE1087

PRE1088

PRE1089

PRE1090

PRE1091

PRE1092

PRE1093

PRE1094

PRE1095

PRE1096

PRE1097

PRE1098

PRE1099

PRE1100

PRE1101

PRE1102

PRE1103

PRE1 104

PRE1105

PRE1106

PRE1107

PRE1108

PRE1109

PRE1110

PRE1111

PRE1112

PRE1113

PRE1114

PRE1115

PRE1116

PRE1117

PRE1118

PRE1119

PRE1120

PRE1121

PRE1122

PRE1123

PRE1 124

PRE1125

PRE1126

PRE1127

PRE1128

PRE1129

PRE1130

PRE1131

PREI 132

PRE1133

PRE1134

PRE1 135

PRE1136

PRE1137

PRE1138

PRE1139 
nuclib $=2$

ELSEIF (name(1:5).EQ. 'CF250') THEN

PRE1140

$\mathrm{m}=250.0+71.167 / 931.5016$

Thal $f=4.128 \mathrm{e} 08$

namy $(1: 6)=' 982500^{\circ}$

nuclib $=2$

ELSEIF (name (1:5) .EO. 'CF251') THEN $\mathrm{m}=251.0+74.129 / 931.5016$

Thalf $=2.834 \mathrm{e} 10$

namy $(1: 6)=\cdot 982510^{\prime}$

nuclib $=2$

ELSEIF (name (1:5).EQ. 'CF252') THEN

$m=252.0+76.03 / 931.5016$

Thalf $=8.325 e 07$

$\operatorname{namy}(1: 5)=982520^{\circ}$

nucl $i b=2$

ELSEIF (name(1:5) .EQ. 'CM242') THEN $m=242.0+54.800 / 931.5016$

Thalf $=1.410 \mathrm{e} 07$

namy $(1: 6)=1962420$. nuclib=2

ELSEIF (name (1:5) .EQ. 'CM243') THEN $\mathrm{m}=243.0+57.177 / 931.5016$

Thalf $=8.994 \mathrm{e} 08$

namy $(1: 6)=' 962430$

nuclib $=2$

ELSEIF (name (1:5) .EO. 'CM244') THEN $\mathrm{m}=244.0+58.449 / 931.5016$

Thalf $=5.715 e 08$

$\operatorname{namy}(1: 6)=' 962440$ '

nuclib $=2$

ELSEIF (name (1:5). EQ. 'CM245') THEN

$m=245.0+60.998 / 931.5016$

Thalf $=2.682 \mathrm{el1}$

$\operatorname{namy}(1: 6)=1962450$ '

nuclib=2

ELSEIF (name (1:5) .EQ. 'CM246') THEN $\mathrm{m}=246.0+62.614 / 931.5016$

Thalf $=1.493 \mathrm{el} 1$

namy $(1: 6)=\cdot 962460$.

nucl $i b=2$

ELSEIF (name (1:5).EO. 'CM248') THEN $\mathrm{m}=248.0+67.388 / 931.5016$

Thal $f=1.070 \mathrm{el}$

namy $(1: 6)=\cdot 962480^{\circ}$

nuclib $=2$

ELSEIF (name (1:4).EO.'CO58') THEN

$\mathrm{m}=58.0-59.844 / 931.5016$

Thalf $=6.115 \mathrm{e} 06$

namy $(1: 6)=\cdot 270580^{\prime}$

nuclib=1

ELSEIF (name (1:4).EQ. 'CO60') THEN $m=60.0-61.646 / 931.5016$

Thalf $=1.663 \mathrm{e} 08$

namy $(1: 6)=\cdot 270600^{\prime}$

nuclib $=1$

ELSEIF (name (1:4).EQ.'CR51') THEN $m=51.0-51.447 / 931.5016$

Thalf $=2.394 \mathrm{e} 06$

namy $(1: 6)=' 240510$ '

nuclib $=1$

ELSEIF (name (1:5). EO. 'CS134') THEN $m=134.0-86.906 / 931.5016$

Thalf $=6.507 \mathrm{e} 07$

namy $(1: 6)=\cdot 551340$ '

nuclib $=1$

ELSEIF (name (1:5). EQ. 'CS135') THEN $m=135.0-87.662 / 931.5016$

Thalf $=7.258 \mathrm{El} 3$

$\operatorname{namy}(1: 6)=\cdot 551350^{\circ}$

nuclib $=1$

ELSEIF (name (1:5) .EO. 'CS137') THEN $m=137.0-86.556 / 931.5016$

Thalf $=9.467 \mathrm{e} 08$

namy $(1: 6)=.551370$.

nuclib=1

PRE1144

PRE1145

PRE1146

PRE1147

PRE1148

PRE1149

PRE1150

PRE1151

PRE1152

PRE1153

PRE1154

PRE1155

PRE1156

PRE 1157

PRE1158

PRE1159

PRE1160

PRE1161

PRE1162

PRE1163

PRE1164

PRE1165

PRE 1166

PRE1167

PRE1168

PRE1169

PRE1170

PRE1171

PRE1172

PRE1173

PRE1174

PRE1175

PRE1176

PRE1177

PRE1178

PRE1179

PRE1180

PRE1181

PRE1182

PRE1183

PRE1184

PRE1185

PRE1186

PRE1187

PRE1188

PRE1189

PRE1190

PRE1191

PRE1192

PRE1193

PRE1194

PRE1195

PRE1196

PRE119?

PRE1198

PRE1199

PRE1200

PRE1201

PRE1202

PRE1203

PRE1204

PRE1205

PRE1206

PRE1207

PRE1208

PRE1209

PRE1210

PRE1211

PRE1212

PRE1213

PRE1214

PRE1215 
ELSEIF (name (1:5) .EQ.'ES253') THEN

$m=253.0+79.007 / 931.5016$

PRE1217

Thalf $=1.769 \mathrm{e} 06$

namy $(1: 6)=\cdot 992530$.

nuclib $=2$

ELSEIF (name (1:5).EQ.'ES254') THEN

IF (name $(6: 6) . E Q . ' M$ ') THEN

$\mathrm{m}=254.0+82.072 / 931.5016$

Thalf $=1.415 \mathrm{e} 05$

namy $(1: 6)=' 992541$ '

ELSE nuclib=2

$\mathrm{m}=254.0+81.994 / 931.5016$

Thalf $=2.382 \mathrm{e} 07$

namy $(1: 6)=\cdot 992540$.

FNDTF

nuclib $=2$

ELSEIF (name (1:5).EO. 'EU150') THEN

$\mathrm{m}=150.0-74.800 / 931.5016$

Thal $f=1.136 \mathrm{e} 09$

namy $(1: 6)=.631500^{\circ}$

nuclib $=3$

ELSEIF (name (1:5) .EO. 'EU152') THEN

$m=152.0-72.899 / 931.5016$

Thalf $=4.292 \mathrm{e} 08$

namy $(1: 6)=' 631520$ '

nuclib $=3$

ELSEIF (name (1:5) .EQ.'EU154') THEN

$\mathrm{m}=154.0-71.748 / 931.5016$

Thalf $=2.714 \mathrm{e} 08$

namy $(1: 6)=' 631540$

nuclib $=3$

ELSEIF (name (1:5).EQ. 'EU155') THEN

$m=155.0-71.829 / 931.5016$

Thalf $=1.565 \mathrm{e} 08$

namy $(1: 6)={ }^{\prime} 631550$ '

nuclib $=3$

ELSEIF (name (1:4).EQ.'FE55') THEN

$m=55.0-57.476 / 931.5016$

Thalf $=8.2048 \mathrm{e} 07$

namy $(1: 6)=' 260550$ '

nuclib $=1$

ELSEIF (name (1:4) . EQ.'FE59') THEN $\mathrm{m}=59.0-60.661 / 931.5016$

Thalf $=3.888 \mathrm{e} 06$

namy $(1: 6)=' 260590^{\circ}$

nuclib $=1$

ELSEIF (name (1:5) .EQ. 'GD162') THEN

$m=162.0-64.240 / 931.5016$

Thalf $=6.000 \mathrm{E} 02$

$\operatorname{namy}(1: 6)=1641620^{\circ}$

nuclib $=1$

ELSEIF (name (1:2).EQ. 'H3') THEN

$\mathrm{m}=3.0+14.95 / 931.5016$

Thal $f=3.897 \mathrm{e} 08$

namy $(1: 6)=\cdot 010030^{\circ}$

nuclib=1

ELSEIF (name (1:4) EQ.'I129') THEN

$m=129.0-88.507 / 931.5016$

Thal $f=4.954 \mathrm{e} 14$

namy $(1: 6)=.531290^{\circ}$

nuclib $=1$

ELSEIF (name (1:4) .EO. 'KR85') THEN $\mathrm{m}=85.0-81.477 / 931.5016$

Thalf $=3.383 \mathrm{e} 08$

namy $(1: 6)=\cdot 360850^{\circ}$

nuclib $=1$

ELSEIF (name (1:4). EQ. 'MN54') THEN

$m=54.0-55.553 / 931.5016$

Thal $f=2.700 \mathrm{e} 07$

namy $(1: 6)=' 250540^{\circ}$

nuclib=1

ELSEIF (name (1:4).EQ.'NB95') THEN

IF (name (5:5).EQ. ' $M$ ') THEN

$m=95.0-86.547 / 931.5016$

Thal $f=3.118 \mathrm{E} 05$

PRE1218

PRE1219

PRE1220

PRE1221

PRE1222

PRE1223

PRE1224

PRE1225

PRE1226

PRE1227

PRE1228

PRE1229

PRE1230

PRE1231

PRE1232

PRE1233

PRE1234

PRE1235

PRE1236

PRE1237

PRE1238

PRE1239

PRE1240

PRE1241

PRE1242

PRE1243

PRE1244

PRE1245

PRE1246

PRE 1247

PRE1248

PRE1249

PRE1250

PRE1253

PRE1252

PRE1253

PRE1254

PRE1255

PRE1256

PRE1257

PRE1258

PRE1259

PRE1260

PRE1261

PRE1262

PRE1263

PRE1264

PRE1265

PRE12 66

PRE1267

PRE1268

PRE1269

PRE1270

PRE1271

PRE 1272

PRE1273

PRE1274

PRE1275

PRE1276

PRE1277

PRE1278

PRE1279

PRE1280

PRE1281

PRE1282

PRE1283

PRE1284

PRE1285

PRE1286

PRE1287

PRE1288

PRE1289

PRE1290

PRE1291 
$\operatorname{namy}(1: 6)=' 410951$ '

PRE1292

nuclib $=1$

ELSE

$m=95.0-86.783 / 931.5016$

PRE1293

PRE1294

PRE1295

Thalf $=3.037 \mathrm{e} 06$

namy $(1: 6)=' 410950$ '

PRE1296

nuclib $=1$

EMNDIE

ELSEIF (name (1:4).EQ. 'NI59') THEN

$\mathrm{m}=59.0-61.153 / 931.5016$

Thal $f=2.5246 \mathrm{e} 12$

$\operatorname{namy}(1: 6)=' 280590$ ' nuclib $=1$

ELSEIF (name (1:4).EQ. 'NI63') THEN

$m=63.0-65.512 / 931.5016$

Thalf $=2.9032 \mathrm{e} 9$

namy $=' 280630^{\circ}$

nuclib $=1$

ELSEIF (name (1:5) .EO. 'NP237') THEN $\mathrm{m}=237.0+44.868 / 931.5016$

Thalf $=6.753 \mathrm{e} 13$

namy $=$ ' 932370 '

nuclib $=2$

ELSEIF (name (1:5) .EQ. 'NP239') THEN

$m=239.0+49.306 / 931.5016$

Thalf $=2.035 \mathrm{e} 05$

namy $=1932390$

nuclib=2

ELSEIF (name (1:5) .EQ. 'PA231') THEN $\mathrm{m}=231.0+33.422 / 931.5016$

Thalf $=1.034 \mathrm{e} 12$

namy $=$ ' $912310^{\circ}$

nuclib $=2$

ELSEIF (name (1:5).EQ. 'PA233') THEN

$\mathrm{m}=233.0+37.485 / 931.5016$

Thalf $=2.333 \mathrm{e} 06$

namy $=\cdot 912330$ '

nucl $1 \mathrm{~b}=2$

ELSEIF (name (1:5).EQ. 'PB210') THEN $\mathrm{m}=210.0-14.752 / 931.5016$

Thal $f=7.037 \mathrm{e} 08$

namy $=$ ' 822100 '

nuclib $=2$

ELSEIF (name(1:5).EQ.'PB214') THEN

$\mathrm{m}=214.0-.188 / 931.5016$

Thal $f=1.608 \mathrm{e} 03$

namy $=$ ' 822140 '

nuclib $=2$

ELSEIF (name (1:5).EQ. 'PD107') THEN $m=107.0-88.374 / 931.5016$

Thalf $=2.050 \mathrm{E} 14$

namy $=$ ' 461070 ' nuclib $=1$

ELSEIF (name (1:5) .EQ.'PM147') THEN $\mathrm{m}=147.0-79.052 / 931.5016$

Thalf $=8.279 e 07$

namy $=$ ' 611470 ' nuclib=1

ELSEIF (name (1:5) .EQ.'PO210') THEN $\mathrm{m}=210.0-15.977 / 931.5016$ Thalf $=1.196 \mathrm{e} 07$ namy $=$ ' 842100 ' nucli $b=2$

ELSEIF (name (1:5).EQ.'PO214') THEN $m=214.0-4.493 / 931.5016$

Thalf $=1.643 \mathrm{e}-04$ namy $=\cdot 842140^{\circ}$ nuclib=2

ELSEIF (name (1:5) .EQ.'PO218') THEN $m=218.0+8.351 / 931.5016$

Thalf $=1.830 \mathrm{e} 02$

namy $=.842180$ ' nuclib $=2$

ELSEIF (name (1:5) .EQ.'PR144') THEN $m=144.0$ - $80.76 / 931.5016$ Thalf $=1.037 \mathrm{e03}$

PRE1298

PRE1299

PRE1300

PRE1301

PRE1302

PRE1303

PRE1304

PRE 1305

PRE1306

PRE 1307

PRE1308

PRE1309

PRE1310

PRE1311

PRE1312

PRE1313

PRE1314

PRE 1315

PRE1316

PRE1 317

PRE1318

PRE1319

PRE1320

PRE1321

PRE1322

PRE1323

PRE1324

PRE1325

PRE1326

PRE1327

PRE1328

PRE1329

PRE1330

PRE1331

PRE1332

PRE1333

PRE1334

PRE1335

PRE1336

PRE1337

PRE 1338

PRE1339

PRE1340

PRE1341

PRE1342

PRE1343

PRE1344

PRE1345

PRE1346

PRE1347

PRE1348

PRE1349

PRE1350

PRE1351

PRE1352

PRE1353

PRE1354

PRE1355

PRE1356

PRE1357

PRE1358

PRE1359

PRE 1360

PRE1361

PRE1362

PRE1363

PRE1364

PRE1365

PRE1366

PRE1367 
namy $=$ ' 591440 '

nuclib $=1$

ELSEIF (name (1:5) .EQ. 'PU236') THEN $\mathrm{m}=236.0+42.879 / 931.5016$

Thalf $=8.997 \mathrm{e} 07$

namy $=$ ' 942360 '

nuclib $=2$

ELSEIF (name (1:5) .EQ.'PU238') THEN

$m=238.0+46.16 / 931.5016$

Thalf $=2.769 \mathrm{e} 09$

namy $=$ ' 942380 '

nuclib $=2$

ELSEIF (name (1:5).EQ.'PU239') THEN $\mathrm{m}=239.0+48.584 / 931.5016$

Thalf $=7.594 \mathrm{e} 11$

namy $=$ ' 942390 '

nuclib=2

ELSEIF (name (1:5) .EQ. 'PU240') THEN $\mathrm{m}=240.0+50.122 / 931.5016$

Thalf $=2.063 \mathrm{el1}$

namy $=1942400$

nuclib $=2$

ELSEIF (name (1:5) .EQ. 'PU241') THEN $\mathrm{m}=241.0+52.952 / 931.5016$

Thalf $=4.544 \mathrm{e} 08$

namy $=$ ' 942410

nuclib $=2$

ELSEIF (name (1:5) .EQ. 'PU242') THEN

$\mathrm{m}=242.0+54.713 / 931.5016$

Thalf $=1.221 \mathrm{e} 13$

namy $=\cdot 942420$

nuclib $=2$

ELSEIF (name (1:5) .EQ.'PU244') THEN $m=244.0+59.802 / 931.5016$

Thalf $=2.607 \mathrm{e} 15$

namy $=$ ' $942440^{\prime}$

nuclib $=2$

ELSEIF (name (1:5).EQ.'RA223') THEN $\mathrm{m}=223.0+17.232 / 931.5016$

Thalf $=9.879 \mathrm{e} 05$

namy $=$ ' $882230^{\circ}$ nuclib $=2$

ELSEIF (name (1:5).EO. 'RA226') THEN $m=226.0+23.662 / 931.5016$

Thalf $=5.049 \mathrm{e} 10$

namy $=$ ' $882260^{\prime}$

nuclib $=2$

ELSEIF (name (1:5).EQ. 'RA228') THEN

$m=228.0+28.936 / 931.5016$

Thalf $=2.1143 \mathrm{e} 08$

namy $=$ ' 882280 '

nuclib $=2$

ELSEIF (name (1:5) .EQ.'RH106') THEN $\mathrm{m}=106.0-86.365 / 931.5016$

Thalf $=29.9$

namy $=$ ' 451060 '

nuclib $=1$

ELSEIF (name (1:5).EQ. 'RN222') THEN $\mathrm{m}=222.0+16.367 / 931.5016$

Thalf $=3.304 \mathrm{e} 05$

namy $=$ ' 862220 ' nuclib $=2$

ELSEIF (name (1:5).EQ. 'RU106") THEN $\mathrm{m}=106.0-86.326 / 931.5016$ Thalf $=3.181 e 07$ namy $={ }^{\prime} 441060^{\prime}$

nuclib=1

ELSEIF (name (1:5).EQ.'SB125') THEN $m=125.0-88.258 / 931.5016$

Thalf $=8.741 \mathrm{e} 07$

namy $=$ ' 511250 '

nuclib=1

ELSEIF (name (1:5).EQ.'SB126') THEN

IF (NAME $(6: 6)$. EQ. 'M') THEN

$m=126.0$ - 86.382/931.5016

Thalf $=1.140 \mathrm{EO} 3$

PRE1368

PRE1369

PRE1370

PRE1371

PRE1372

PRE1373

PRE1374

PRE1375

PRE1376

PRE 1377

PRE1378

PRE1379

PRE 1380

PREI 381

PRE1382

PRE1383

PRE1384

PRE1385

PRE1386

PRE1387

PRE1388

PRE1389

PRE1390

PRE1391

PRE1392

PRE1393

PRE1394

PRE1395

PRE1396

PRE1397

PRE1398

PRE1399

PRE1400

PRE1401

PRE1402

PRE1403

PRE1404

PRE1405

PRE1406

PRE1407

PRE1408

PRE1409

PRE1410

PRE1411

PRE1412

PRE1413

PRE1414

PRE1415

PRE1416

PRE1417

PRE1418

PRE1419

PRE1420

PRE142I

PRE1422

PRE1 423

PRE1424

PRE1 425

PRE1426

PRE1427

PRE1428

PRE1429

PRE1430

PRE1431

PRE1432

PRE1433

PRE1434

PRE1435

PRE1436

PRE1 437

PRE1 438

PRE1439

PRE1440

PRE1441

PRE1442

PRE1443 
namy $=' 511261^{\prime}$

nuclib $=1$

ELSE

$m=126.0-86.400 / 931.5016$

Tha $1 £=1.071 \mathrm{E} 06$

namy $=' 511260$

nuclib $=1$

ENDIF

ELSEIF (name (1:4).EQ. 'SE79') THEN

$m=79.0-75.920 / 931.5016$

Thalf $=2.050 \mathrm{E} 12$

namy $=\cdot 340790$

nuclib $=1$

ELSEIF (name (1:5).EQ. 'SM151') THEN

$m=151.0-74.587 / 931.5016$

Thalf $=2.840 \mathrm{e} 09$

namy $=' 621510$ nuclib $=1$

ELSEIF (name $(1: 6)$.EO. 'SN119M') THEN $m=119.0-89.978 / 931.5016$

Thal $f=2.117 \mathrm{E} 07$

namy $=$ ' 501191

nuclib=1

ELSEIF (name $(1: 6) . \mathrm{EQ}$. 'SN121M') THEN

$m=121.0-89.197 / 931.5016$

Thalf $=1.577 \mathrm{E} 09$

namy $=$ '501211'

nuclib $=1$

ELSEIF (name (1:6).EQ. 'SN123M') THENN $m=123.0-87.795 / 931.5016$

Thalf $=2.405 E 03$

namy = ' 501231 .

nuclib $=1$

ELSEIF (name $(1: 5)$.EQ. 'SN126') THEN $\mathrm{m}=126.0-86.021 / 931.5016$

Thalf $=3.156 \mathrm{E} 12$

namy $=$ ' $501260^{\circ}$ nuclib $=3$

ELSEIF (name (1:4) .EQ. 'SR89') THEN $\mathrm{m}=89.0-86.211 / 931.5016$

Thalf $=4.363 e 06$

namy $=$ ' 380890

nuclib $=1$

ELSEIF (name (1:4).EQ.'SR90') THEN

$m=90.0-85.942 / 931.5016$

Thalf $=9.190 \mathrm{e} 08$

namy $=\cdot 380900$

nuclib=1

ELSEIF (name (1:5) .EQ. 'TA182') THEN

$\mathrm{m}=182.0-46.436 / 931.5016$

Thalf $=9.936 \mathrm{e} 06$

namy $=\cdot 731820$

nuclib $=1$

ELSEIF (name (1:5).EQ. 'TB162') THEN

$m=162.0-65.680 / 931.5016$

Thalf $=4.482 \mathrm{EO} 2$

namy $=\cdot 651620$ nuclib=1

ELSEIF (name $(1: 4)$.EO. 'TC99') THEN $\mathrm{m}=99.0-87.324 / 931.5015$

Thalf $=6.722 \mathrm{e} 12$

namy $=\cdot 430990$

nuclib=1

ELSEIF (name $(1: 6) . E Q$. 'TE125M') THEN $\mathrm{m}=125.0-88.879 / 931.5016$

Thalf $=5.011 \mathrm{e} 06$

namy $=' 521251$

nuclib $=1$

ELSEIF (name (1:5) .EQ.'TE127') THEN

IF (NAME (6:6) . EO. ' $M$ ') THEN

$\mathrm{m}=127.0-88.198 / 931.5016$

Thalf $=9.418 \mathrm{E} 06$

namy $=\cdot 521271$

nuclib $=1$

ELSE

$m=127.0-88.286 / 931.5016$

PRE1444

PRE1445

PRE1446

PRE1447

PRE1448

PRE1449

PRE1450

PRE1451

PRE1452

PRE1453

PRE1454

PRE1455

PRE1456

PRE1457

PREI 458

PRE1459

PRE1460

PRE1461

PRE1462

PRE1463

PRE1464

PRE1465

PRE1466

PRE1467

PRE1468

PRE1469

PRE1470

PRE1471

PRE1472

PRE1473

PRE1474

PRE1475

PRE1476

PRE1477

PRE1478

PRE1479

PRE1480

PRE1481

PRE1482

PRE1483

PRE1484

PRE1485

PRE1486

PRE1487

PRE1488

PRE1489

PRE1490

PRE1491

PRE1492

PRE1493

PRE1494

PRE1495

PRE1496

PRE1497

PRE1498

PRE1499

PRE1500

PRE1501

PRE1502

PRE1503

PRE1504

PRE1505

PRE1506

PRE1507

PRE1508

PRE1509

PRE1510

PRE1511

PRE1512

PRE1513

PRE1514

PRE1515

PRE1516

PRE1517

PRE1518

PRE1519 
Thalf $=3.366 \mathrm{E} 04$

PRE1520

namy $=$ ' 521270 '

PRE1521

nuclib $=1$

ENDIF

ELSEIF (name (1:5) .EQ. 'TH228') THEN $\mathrm{m}=228.0+26.749 / 931.5016$

Thalf $=6.037 \mathrm{e} 07$

namy $=$ ' $902280^{\circ}$

nuclib $=2$

ELSEIF (name (1:5) .EQ.'TH230') THEN $\mathrm{m}=230.0+30.858 / 931.5016$

Thalf $=2.430 \mathrm{e} 12$

namy $=$ ' 902300 ' nuclib $=2$

ELSEIF (name (1:5).EO. 'TH232') THEN $\mathrm{m}=232.0+35.444 / 931.5016$

Thalf $=4.434 \mathrm{el}$

namy $=.90232 .0^{\circ}$

nuclib $=2$

ELSEIF (name (1:5) .EQ.'TH234') THEN

$\mathrm{m}=234.0+40.607 / 931.5016$

Thalf $=2.082 \mathrm{e06}$

namy $=\cdot 902340$

nuclib $=2$

ELSEIF (name (1:4) .EQ. 'U232') THEN

$m=232.0+34.587 / 931.5016$

Thalf $=2.272 \mathrm{e} 09$

namy $=\cdot 922320^{\circ}$

nucli $b=2$

ELSEIF (name (1:4).EQ. 'U233') THEN $\mathrm{m}=233.0+36.915 / 931.5016$

Thalf $=5.002 \mathrm{e} 12$

namy $=$ ' $922330^{\circ}$

nucli $b=2$

ELSEIF (name (1:4).EQ.' U234') THEN $\mathrm{m}=234.0+38.141 / 931.5016$

Thalf $=7.716 \mathrm{e} 12$

namy $=\cdot 922340$

nuclib $=2$

ELSEIF (name (1:4).EQ.'U235') THEN

$\mathrm{m}=235.0+40.915 / 931.5016$

Thalf $=2.221 \mathrm{e} 16$

namy $=.922350$

nuclib $=2$

ELSEIF (name $(1: 4) . E Q . ' U 236$ ') THEN

$\mathrm{m}=236.0+42.441 / 931.5016$

Thalf $=7.389 \mathrm{e} 14$

namy $=.922360$

nucli $b=2$

ELSEIF (name (1:4).EQ.'U238') THEN $\mathrm{m}=238.0+47.305 / 931.5016$

Thalf $=1.410 \mathrm{el}$

namy $=.922380^{\circ}$ nuclib $=2$

ELSEIF (name (1:3) .EQ. 'Y90') THEN $\mathrm{m}=90.0-86.488 / 931.5016$

Thalf $=2.304 \mathrm{e} 05$

namy $=$ ' 390900 nuclib $=1$

ELSEIF (name (1:4) EO.'ZN65') THEN $m=65.0-65.91 / 931.5016$

Thalf $=2.107 \mathrm{e} 07$

namy $=$ ' 300650

nuclib $=1$

ELSEIF (name (1:4).EQ.'ZR93') THEN

$m=93.0-87.120 / 931.5016$

Thal $f=4.828 \mathrm{E} 13$

namy $=1400930$

nuclib $=1$

ELSEIF (name (1:4).EQ.'ZR95') THEN $m=95.0-85.659 / 931.5016$

Thal $f=5.528 \mathrm{e} 06$

namy $=' 400950^{\prime}$

ELSE nuclib $=1$

$\mathrm{m}=.5$

PRE1522

PRE1523

PRE1524

PRE1525

PRE1526

PRE1527

PRE1528

PRE1529

PRE1530

PRE1531

PRE1532

PRE1533

PRE1534

PRE1535

PRE1536

PRE1537

PRE1538

PRE1539

PRE1540

PRE1541

PRE1542

PRE1543

PRE1544

PRE1545

PRE1546

PRE1547

PRE1548

PRE1549

PRE1550

PRE1551

PRE1552

PRE1553

PRE1554

PRE1555

PRE1556

PRE1557

PRE1558

PRE1559

PRE1560

PRE1561

PRE1562

PRE1563

PRE1564

PRE1565

PRE1566

PRE1567

PRE1568

PRE1569

PRE1570

PRE1571

PRE1572

PRE1573

PRE1574

PRE1575

PRE1576

PRE1577

PRE1578

PRE1579

PRE1580

PRE1581

PRE1582

PRE1583

PRE1584

PRE1585

PRE1586

PRE1587

PRE1588

PRE1589

PRE1590

PRE1591

PRE1592

PRE1593

PRE1594

PRE1595 


\author{
Thalf $=.05$ \\ namy $=\cdot * * \star * \star *$. \\ ENDIF \\ nuclib $=0$
}

C FROM THE BOOK "ATOMS, RADIATION, AND RADIATION PROTECTION"

$C$ BY JAMES TURNER, USED A FORMULA TO CALCULATE SPECIFIC

C ACTIVITY IN BQ/G WHEN THE HALFLIFE WAS EXPRESSED IN SECONDS. C

C THEN CONVERTED Bq/g TO $\mathrm{Ci} / \mathrm{g}$

$$
s a 1=(6.022136736 \mathrm{e} 23 * \operatorname{alog}(2.0)) /(\mathrm{m} * \text { Thal } f)
$$

$$
\mathbf{s a}=\operatorname{sa1} / 3.7 \mathrm{e} 10
$$

C IT IS THEN POSSIBLE TO CONVERT A VALUE IN CURIES TO GRAMS

C BY THE FOLLOWING EQUATION.$$
\text { C }
$$

$c$

$$
\text { activy }=\operatorname{activ} / \mathbf{s a}
$$

\section{RETURN}

END

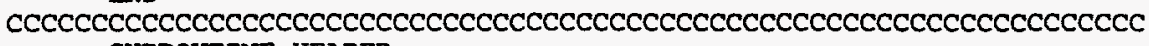
SUBROUTINE HEADER

c WRITE $(6,9801)$ 9801 FORMAT (

$+\quad 1234567890123456789012345678901234567890^{\circ}$. +1,

$+$

$+1, \cdot$

1

$+1$.

$1, \cdot$

$+$

$+1, \cdot$

$+1 .+>$

$+1,1>$

$+1,1 \quad>$

$+1,1>$

$+1$

$\operatorname{WRITE}(6,9802)$

9802 FORMAT (

$+\quad>$

$+$

$+1, \cdot \quad>$

$+1 \cdot 0>$

$+1, \quad>>$

$+1, \cdot$

$\gg>$

+1,

$+$

$+1, \cdot$

$+10^{\circ}$

+1,

+1,

$+$

$+1$

WRITE $(6,9803)$

9803 FORMAT '

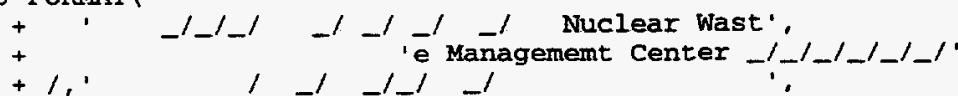

PRE1596

PRE1597

PRE1598

PRE1599

PRE1600

PRE1601

PRE1602

PRE1603

PRE1604

PRE1605

PRE1606

PRE1607

PRE1608

PRE1609

PRE1610

PRE1611

PRE1612

PRE1613

PRE1614

PRE1615

PRE1616

PRE1617

PRE1618

PRE1619

PRE1620

PRE1621

PRE1622

PRE1623

PRE1624

PRE1625

PRE1626

PRE1627

PRE1628

PRE1629

PRE 1630

PRE1631

PRE1632

PRE1633

PRE1634

PRE1635

PRE1636

PRE 2637

PRE1638

PRE1639

PRE1640

PRE1641

PRE1642

PRE1643

PRE1644

PRE1645

PRE1646

PRE1647

PRE1648

PRE1649

PRE1650

PRE1651

PRE1652

PRE1653

PRE1654

PRE1655

PRE1656

PRE1657

PRE1658

PRE1659

PRE1660

PRE1661

PRE1662

PRE1663

PRE1664

PRE1665

PRE1666

PRE1667

PRE1668

PRE1669

PRE1670

PRE1671 

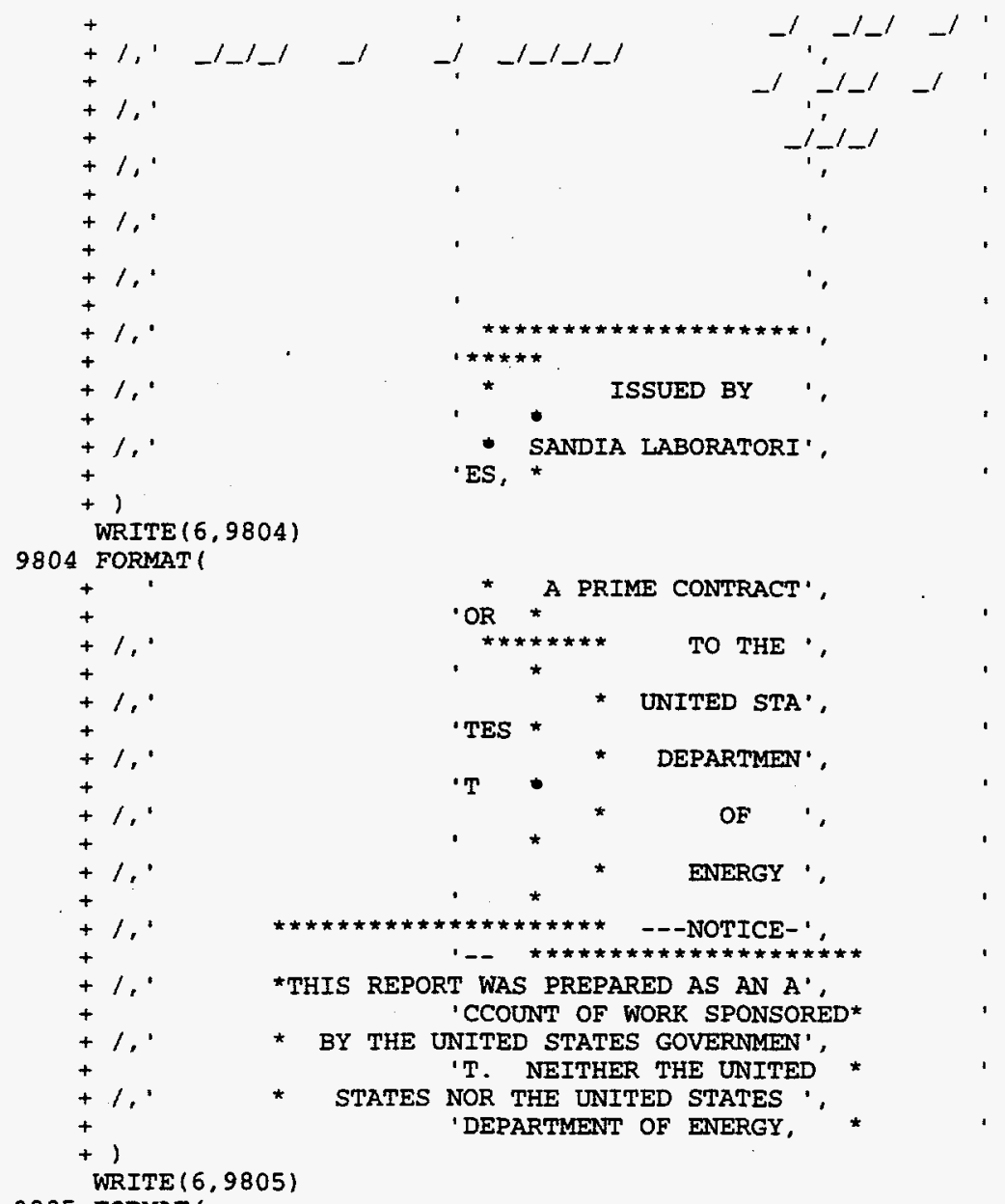

9805 FORMAT!

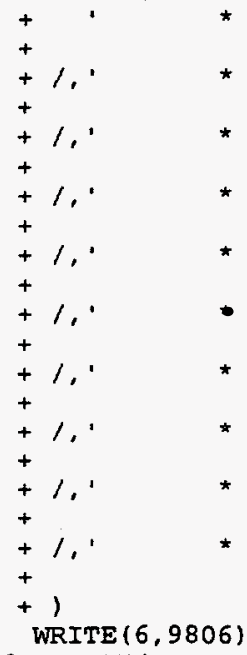

9806 FORMAT (

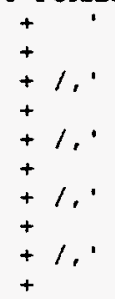

NOR ANY OF THEIR ', ' EMPLOYEES,

* NOR ANY OF THEIR CONTRACTORS, $s$ '

' UBCONTRACTORS, OR THEIR *

* EMPLOYEES, MAKES ANY WARRANTYY, 'EXPRESS OR IMPTIED OR' *

ANY LEGAL LIABILITY OR ? 'RESPONSIBILITY FOR THE $\star * \star * * * \star * * *$ ACCURACY'<smiles>[VH3]</smiles>

'. 'ss

*

'ESS *

$$
\text { * }
$$

*

'N,

,

* COMPLETENE'

* OR USEFULN

*

OF ANY

* INFORMatio.

* apparatus ' .

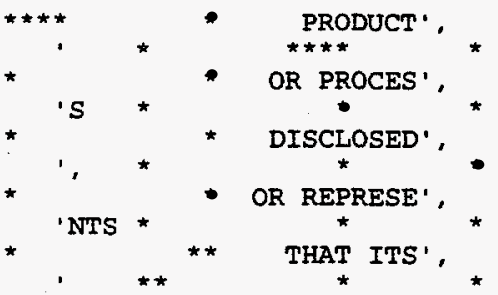

PRE1672

PRE1673

PRE1674

PRE1675

PRE1676

PRE1677

PRE1678

PRE1679

PRE1680

PRE1681

PRE1682

PRE1683

PRE1684

PRE1685

PRE1686

PRE1687

PRE1688

PRE1689

PRE1690

PRE1691

PRE1692

PRE1693

PRE1694

PRE1695

PRE1696

PRE1697

PRE1698

PRE1699

PRE1700

PRE1701

PRE1702

PRE1703

PRE1704

PRE1705

PRE1706

PRE1707

PRE1708

PRE1709

PRE1710

PRE1711

PRE1712

PRE1713

PRE1714

PRE1715

PRE1716

PRE1717

PRE1718

PRE1719

PRE1720

PRE1721

PRE1722

PRE1723

PRE1724

PREI725

PRE1726

PRE1727

PRE1728

PRE1729

PRE1730

PRE1731

PRE1732

PRE1733

PRE1734

PRE1735

PRE1736

PRE1737

PRE1738

PRE1739

PRE1 740

PRE1741

PRE1742

PRE1743

PRE1744

PRE1745

PRE1746

PRE1747 


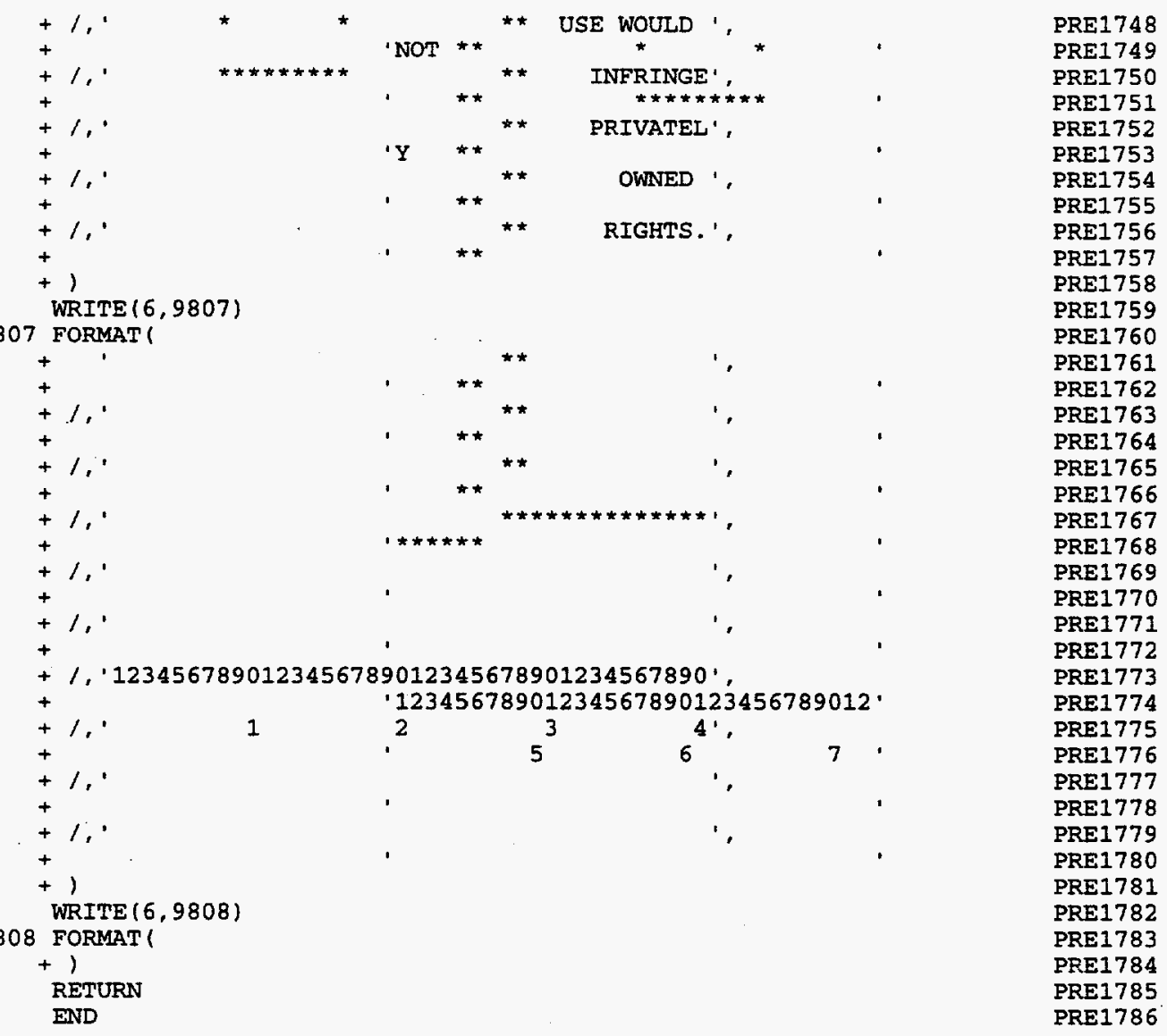




\section{APPENDIX E: PERSONAL COMMUNICATION WITH SAYAN CHAKRABORTI OF}

SCIENCE APPLICATIONS INTERNATIONAL CORPORATION BY JENNIFER POWELL ON JULY 26, 1995 
Intentionally Left Blank 


\section{Sandia National Laboratories}

Albuquerque, New Mexico $87185-1175$

date: May 13, 1996

to: FILE

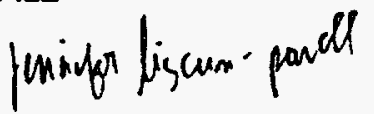

from: Jennifer Liscum-Powell

re: Activity Factors for Mixed Fission Products and Mixed Activtation Products in TWBIR Input Data Files for PreORG

The activity factors for TBGMFP (Mixed Fission Products) and TBGMAP (Mixed Activation Products) were obtained from Sayan Chakraborti of SAIC on July 26, 1995. Sayan is the TWBIR Technical Lead and a Senior Engineer with SAIC. These isotopic compositions are based on the Hanford generation site data.

The activity factors are used to translate activities given in generation sites for the isotope name TBGMAP and TBGMFP into actual isotopic compositions. The activity for an isotope is obtained by multiplying the activity of TBGMAP or TBGMFP, respectively, by the appropriate activity factor as given in Tables 1 and 2 below.

The activity factors are attached in the following tables. A copy of the original fax is also attached. 
FILE

-2 -

May 13, 1996

Table 1. Activity Factors for TBGMAP - Mixed Activation Products

\begin{tabular}{|c||c||}
\hline ISOTOPE & $\begin{array}{c}\text { ACTIVITY } \\
\text { FACTOR }\end{array}$ \\
\hline Ba137m & $9.7000 \mathrm{E}-03$ \\
C14 & $1.0000 \mathrm{E}-02$ \\
Co58 & $1.0000 \mathrm{E}-02$ \\
Co60 & $6.7000 \mathrm{E}-01$ \\
Cs137 & $1.0300 \mathrm{E}-02$ \\
Eu152 & $1.0000 \mathrm{E}-02$ \\
Eu154 & $8.5000 \mathrm{E}-02$ \\
Eu155 & $8.5000 \mathrm{E}-02$ \\
Fe59 & $4.0000 \mathrm{E}-02$ \\
Mn54 & $7.0000 \mathrm{E}-02$ \\
\hline
\end{tabular}


Table 2. Activity Factors for TBGMFP - Mixed Fission Products

\begin{tabular}{|c||c||}
\hline IsoToPE & ACTIVIT \\
FACTOR
\end{tabular}


Appendix E: Personal Communication with Sagan Chakraborti . . . July 26, 1995

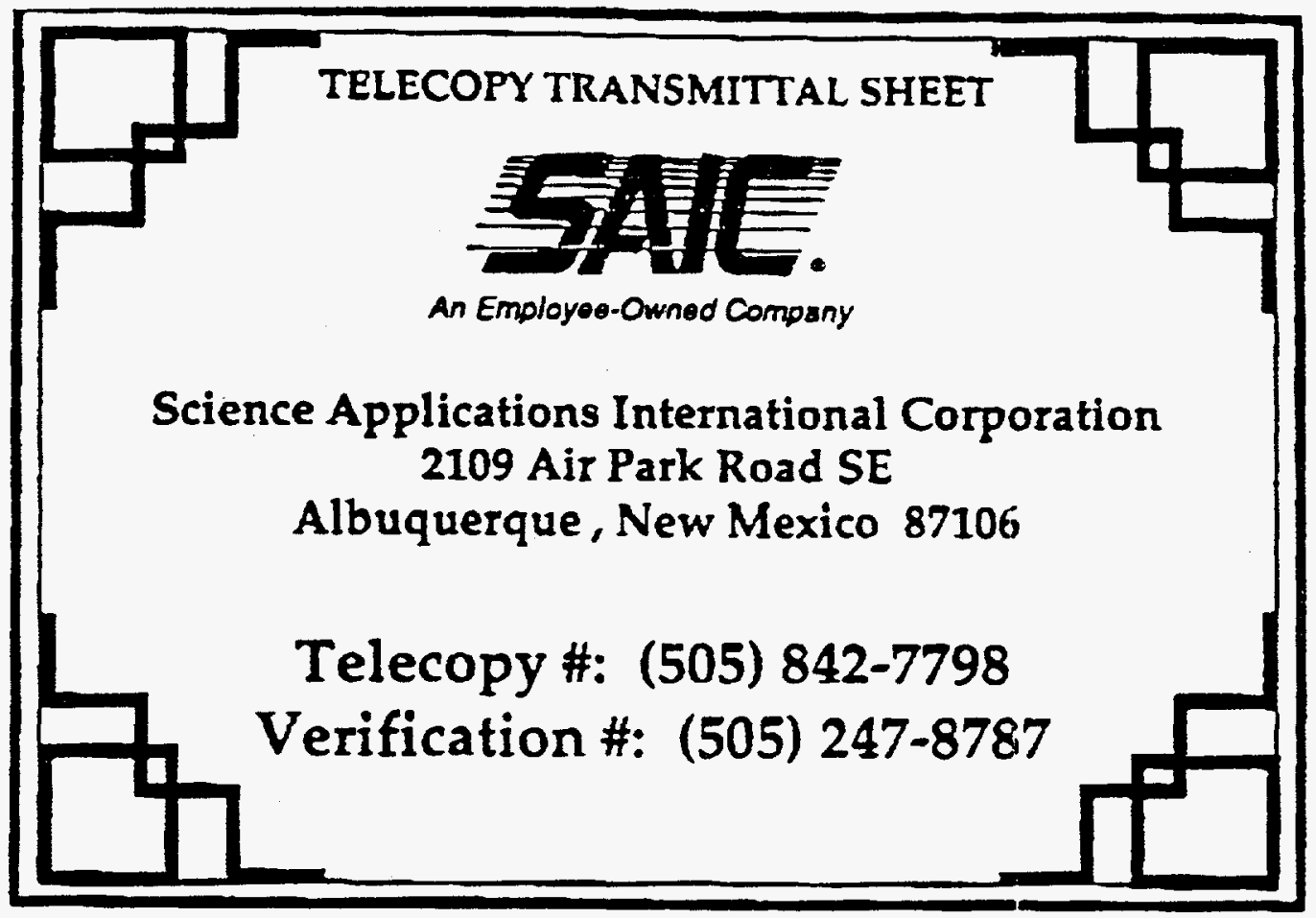

Date: $7 / 26$ Time: $9: 30 \mathrm{am}$ 5-Digit Access Code:

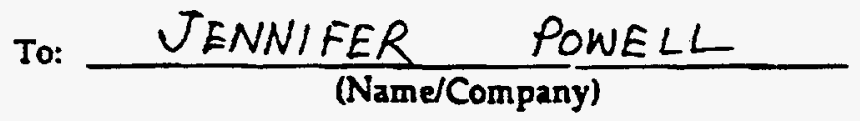

1.

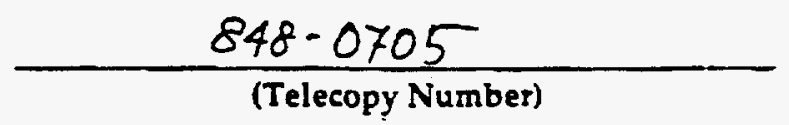

(Verification Number)

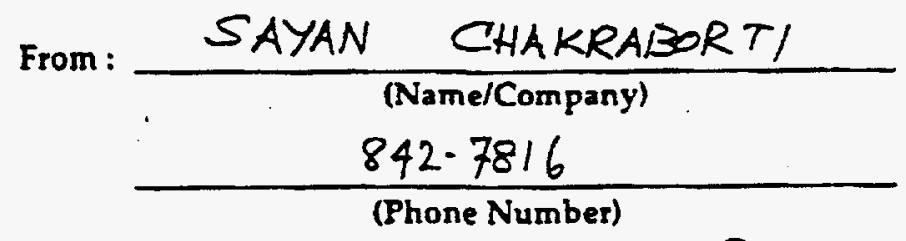

Additional Information: The ratios for the activation
conducts ares given on the right side lower corner of the atiaclee page.

\# of Pages to Follow:

1

E-6 


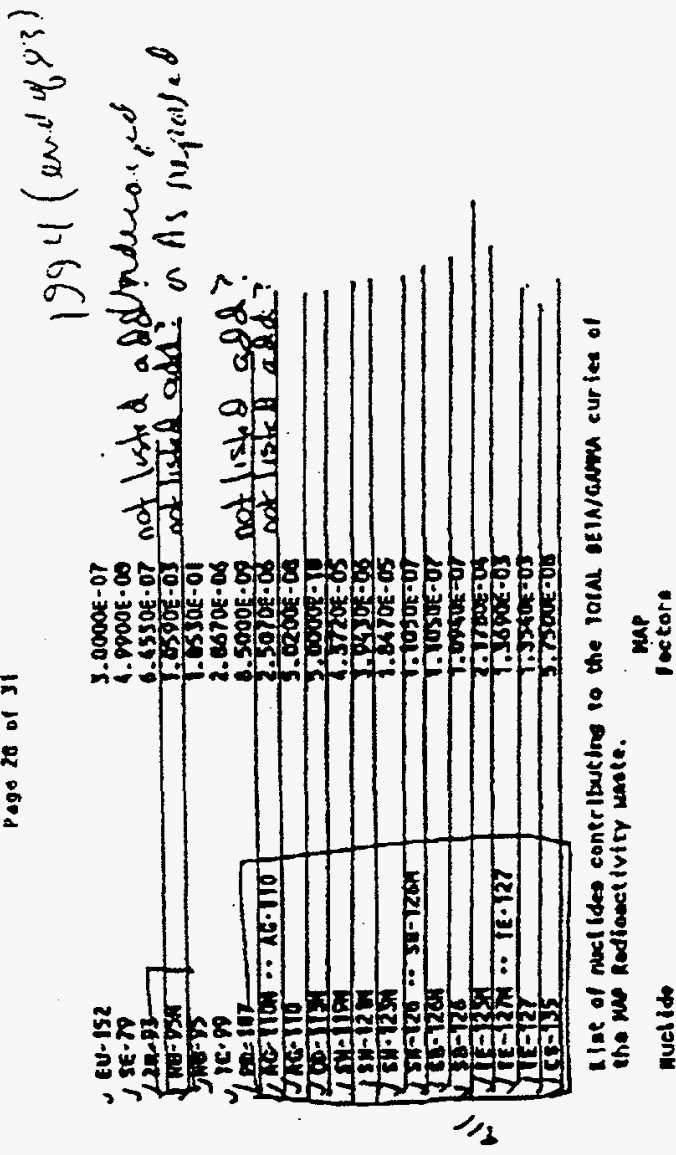

Nㅜㅇ후웡무웅무ㅇㅜㅜ

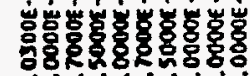

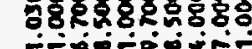

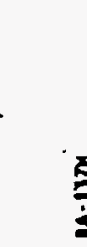

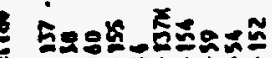

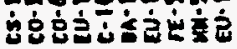

in

$r$

$\infty d$

$\sigma$

I.:

$=t$

$+5$

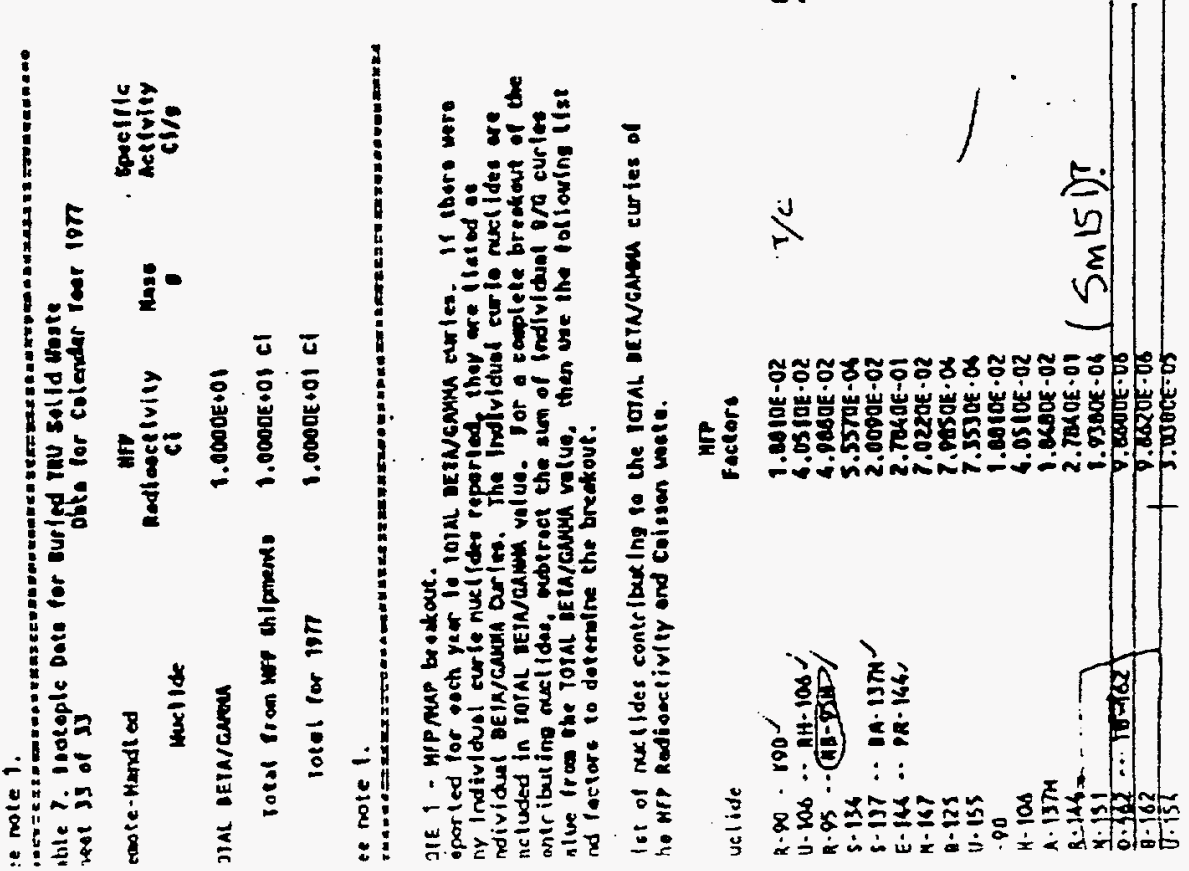


Intentionally Left Blank 
APPENDIX F: OR3_POSTORG.FOR PROGRAM LISTING 
Intentionally Left Blank 
program postorg

POST0001 C23456789012345678901234567890123456789012345678901234567890123456789012POST0002

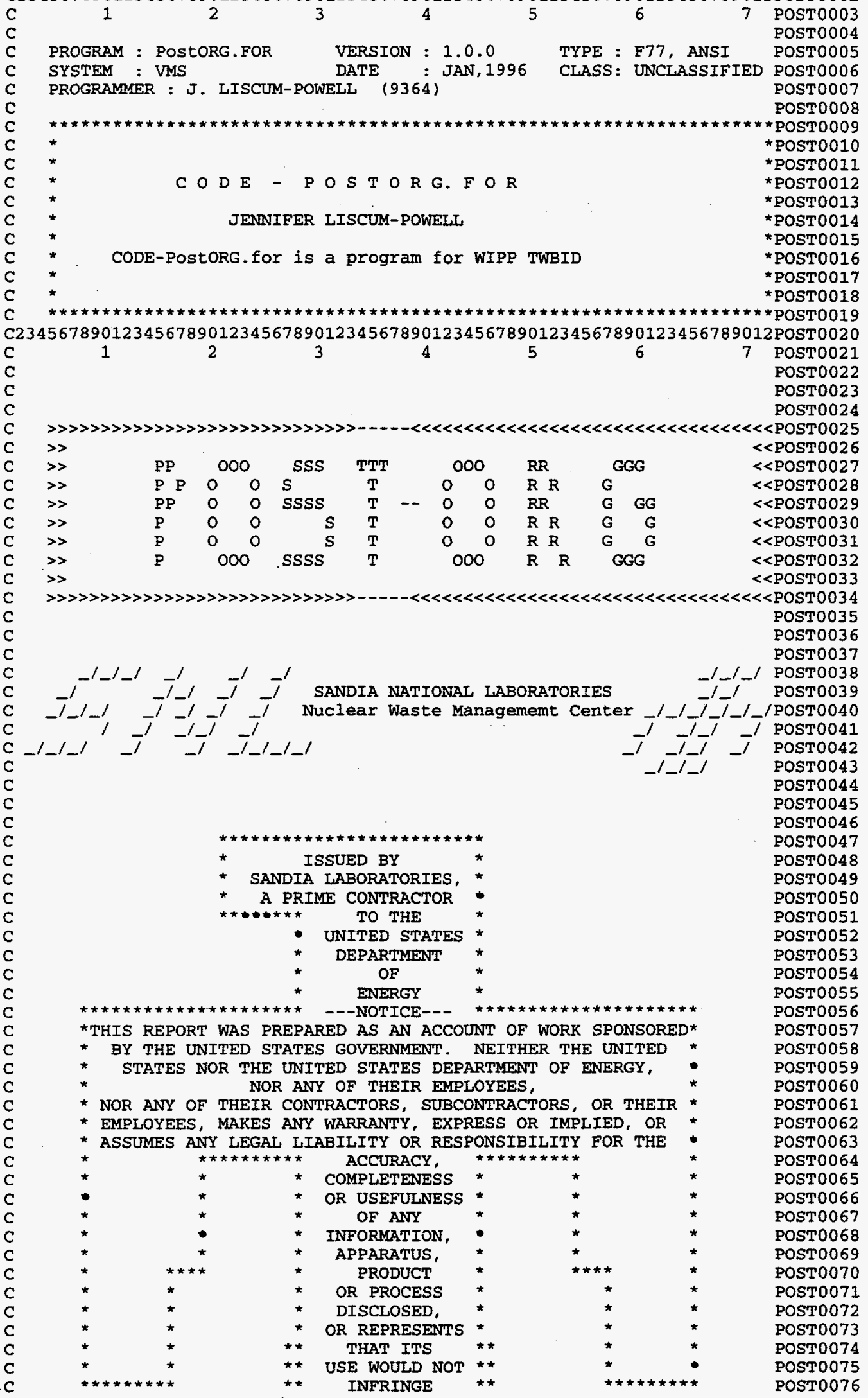




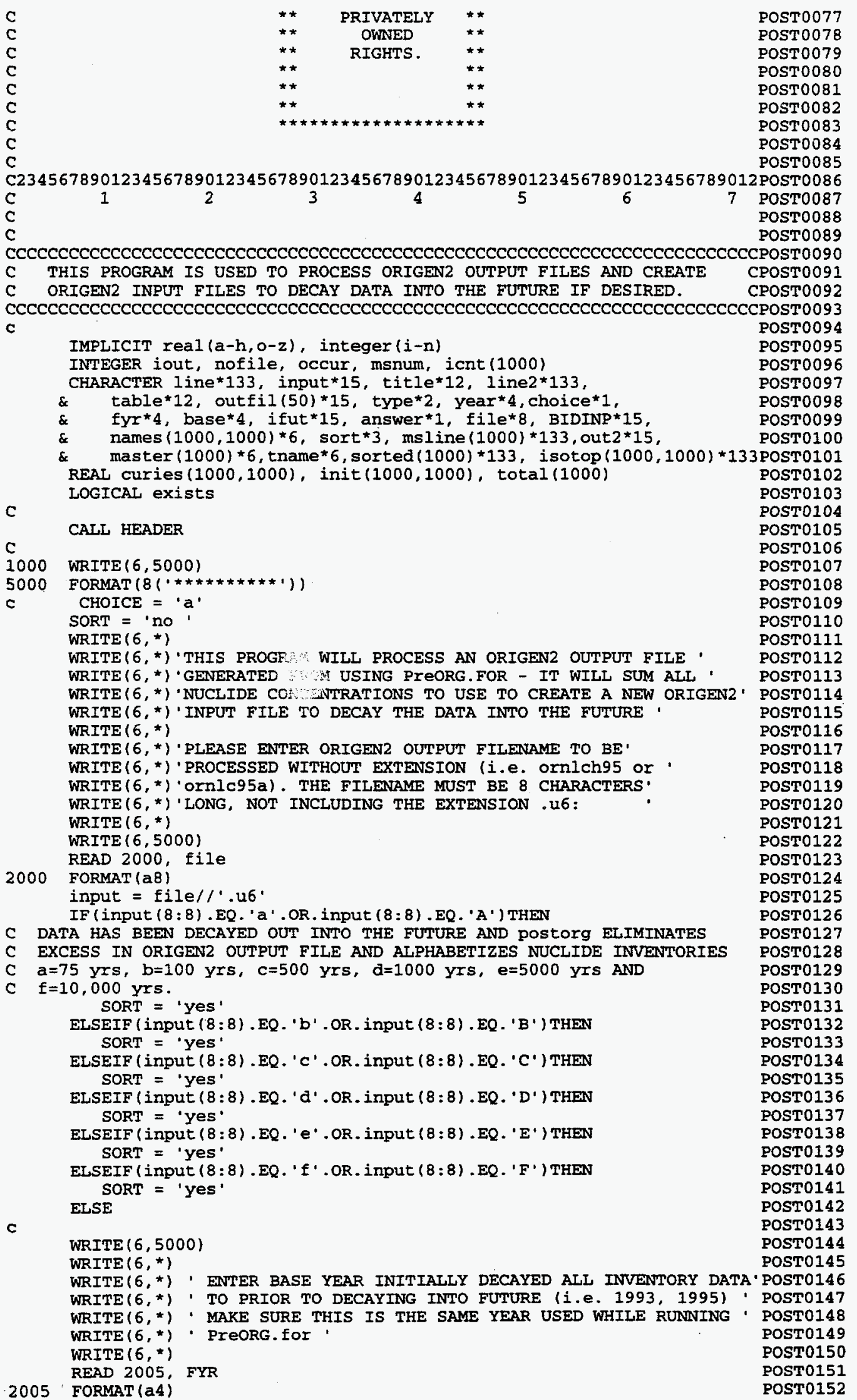


WRITE $(6,5000)$

WRITE $(6$, *

$\operatorname{WRITE}(6, *)$

WRITE $(6, *)$ 'WOULD YOU LIKE TO CREATE AN ORIGEN2 INPUT FILE'

WRITE $(6,5005)$ fyr

WRITE $(6, *)$ $Y$ (YES) or $n$ (NO)

2010 FORMAT (a)

WRITE $(6,5000)$

IF (answer. EQ. ' $Y$ ' . OR . answer . EQ. ' $Y$ ') THEN ENDIF

CALL FUTURE (time, tstep)

ENDIF

$c$

table $=$ input $(1: 8) / /$. ind

C INQUIRE TO SEE IF DATA FILE EXISTS

INQUIRE (FILE=input, EXIST=exists)

IF (.NOT . exists) THEN

C ERROR MESSAGE - INPUT FILE NOT FOUND

WRITE $(6,5010)$ input

\&

FORMAT ( $/, 7(" \star \star E R R O R '), /, /, t 10$,' INPUI FILE ',a12,

'DOES NOT EXIST ', $/, 1,7(1 * \star$ ERROR' $)(1)$

WRITE $(6, *)$ ' TO RE-ENTER FILENAME ENTER [1] OR '

WRITE $(6, *)$, TO EXIT POSTORG ENTER [2]:

$\operatorname{WRITE}(6, *)$

READ*, ians

IF (ians . EQ . 1) THEN

WRITE $(6, *) \cdot$ CHOOSE ANOTHER SITE: ' GO TO 1000

ELSEIF (ians. EQ.2) THEN

GO TO 9999

ENDIF

ENDIF

$\mathrm{C}$

OPEN (UNIT $=10$, FILE $=$ inpLT, STATUS $=$ ' OLD')

OPEN (UNIT $=15$, FILE=table, STATUS = ' NEW')

cont $=0$

bent $=0$

acnt $=0$

ycnt $=0$

lent $=0$

nofile $=0$

WRITE $\left(6,{ }^{*}\right)$

WRITE $(6, *)$ ' ELIMINATING EXCESS DATA FROM ORIGEN2 OUTPUT IF (sort. NE. 'yes') then

WRITE $(6, *)$ CREATING OUTPUT DATAFILE FOR EACH YEAR

WRITE $(6, *)$

$1005 \operatorname{READ}(10,2015$, end $=1020)$ line

2015 FORMAT (a133)

c

IF ( Iine $(13: 14)$.EO. ' 19'. OR. Iine $(13: 13)$.EO. ' ' ' . OR.

\& line $\left.(13: 14), E Q \cdot 0^{\prime} t==^{\prime}\right)$ THEN

IF (sort. EQ. 'Yes' . AND. acnt. EQ.0) THEN

acnt $=1$

OUT2 $=\operatorname{INPUT}(1: 8) / 1$. tot .

OREN (UNIT $=20$, FILE $=$ OUT 2 , STATUS $=$ ' NEW' '

WRITE $(20, *) \cdot$ RADIOACTIVITY IN Curies .

WRITE $(20, *)$

WRITE $(20,5015)$ line

5015 FORMAT (a133)

C

ELSEIF (acnt . NE . 1) THEN

acnt $=1$

type $=\operatorname{input}(5: 6)$

close (unit=iout)

c

READ(line,2020) year, base

POST0153

POST0154

POST0155

POST0156

POST0157

POST0158

POST0159

POST0160

POST0161

POST0162

POST0163

POST0164

POST0165

POST0166

POST0167

POST0168

POST0169

POST0170

POST0171

POST0172

POST0173

POST0174

POST0175

POST0176

POST0177

POST0178

POST0179

POST 0180

POST0181

POST0182

POST0183

POST0184

POST0185

POST0186

POST0187

POST0188

POST0189

POST0190

POST0191

POST0192

POST0193

POST0194

POST0195

POST0196

POST0197

POST0198

POST0199

POST0200

POST0201

POST0202

POST0203

POST0204

POST0205

POST0206

POST0207

POST0208

POST0209

POST0210

POST0211

POST0212

POST0213

POST0214

POST0215

POST0216

POST0217

POST0218

POST0219

POST0220

POST0221

POST0222

POST0223

POST0224

POST0225

POST0226

POST0227

POST0228 


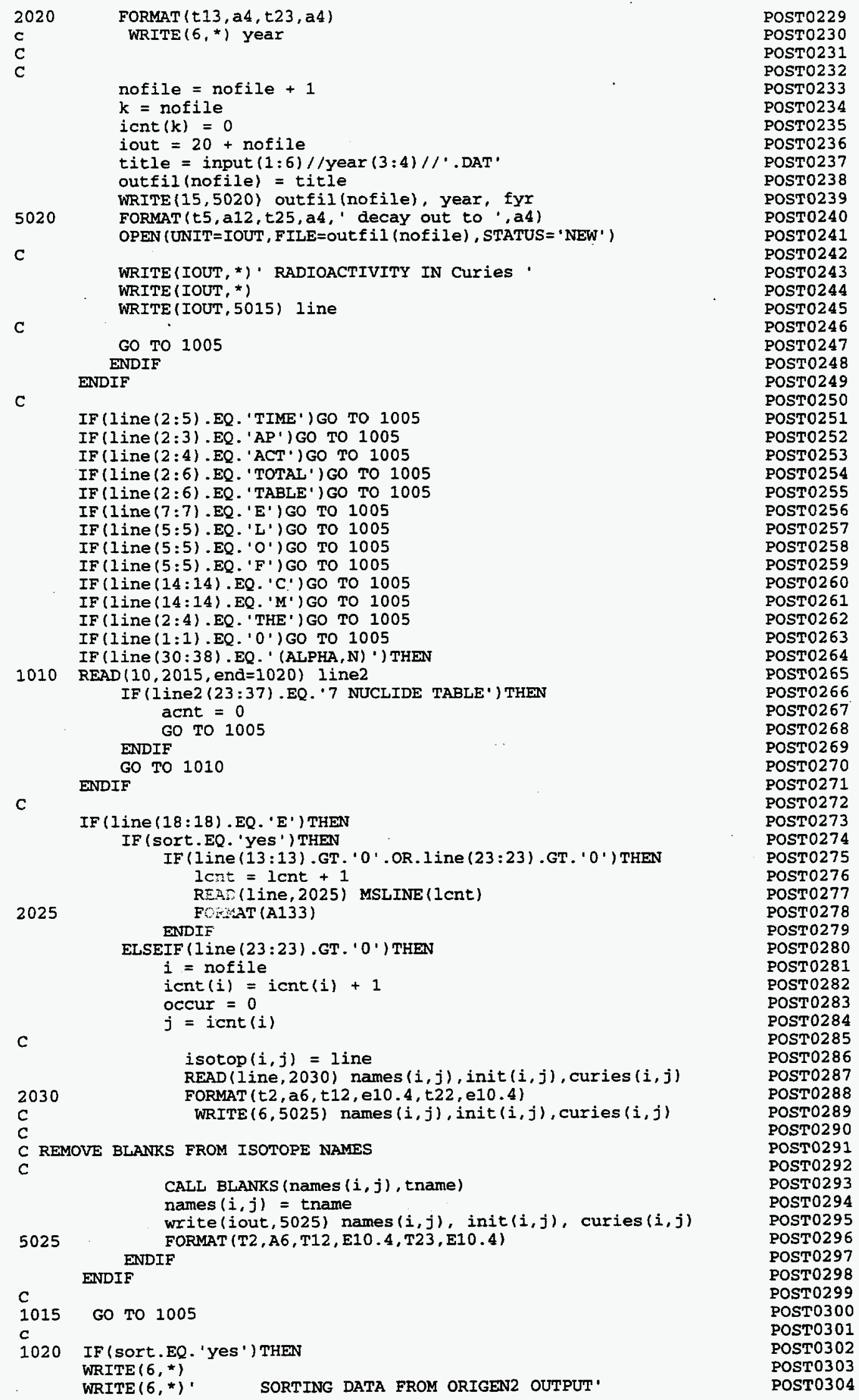




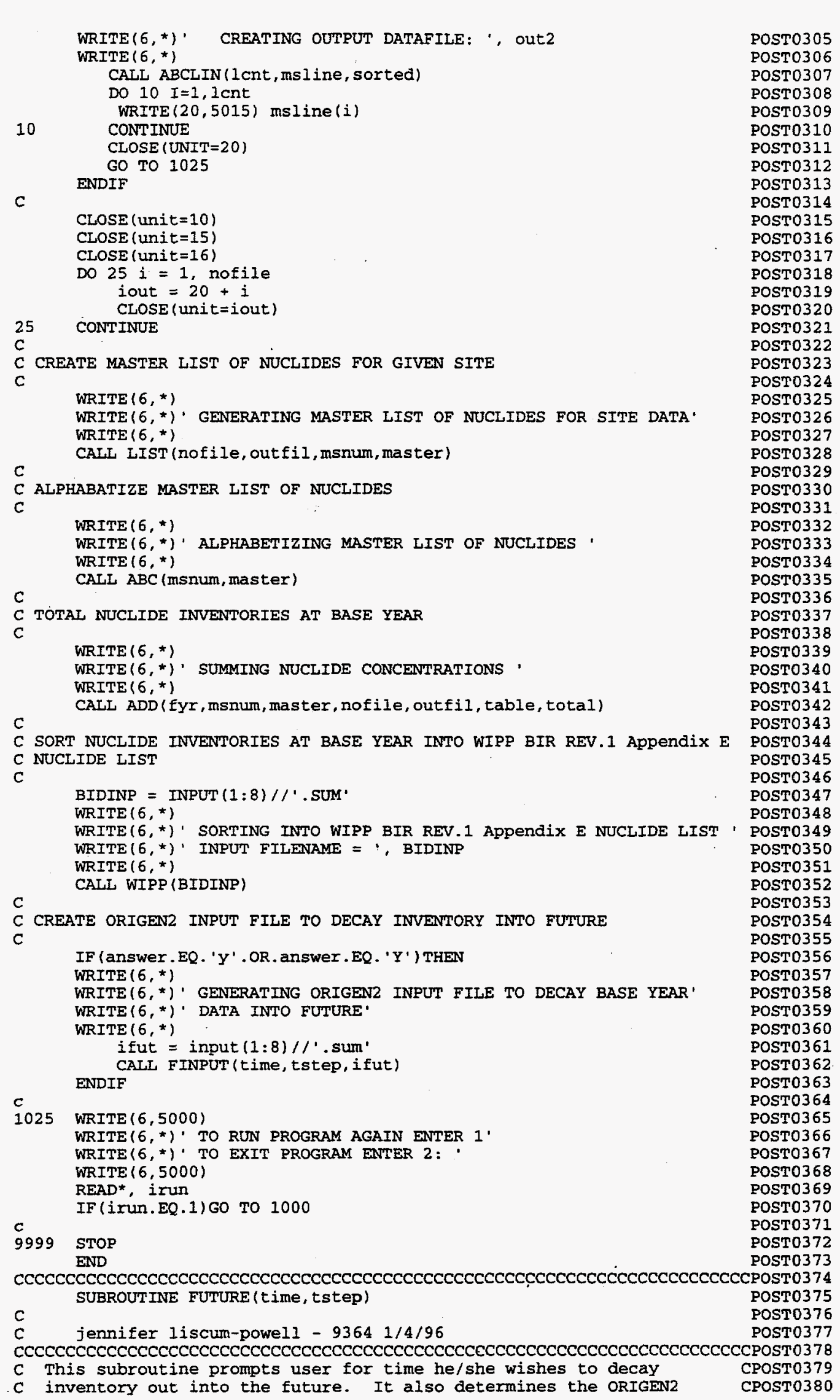


C input commands needed in the ORIGEN2 input file to perform the CPOST0381 C desired calculation CPOST0382 CCCCCCCCCCCCCCCCCCCCCCCCCCCCCCCCCCCCCCCCCCCCCCCCCCCCCCCCCCCCCCCCCCCCCCCCPOSTO 383 IMPLICIT REAI $(a-h, 0-z)$, INTEGER $(i-n) \quad$ POST0384 CHARACTER choice* 1

REAI time, tstep

C PROMPT USER FOR DESIRED TIME TO DECAY INVENTORY INTO FUTURE

C

WRITE $(6, *)$. PLEASE CHOOSE HOW LONG INTO FUTURE YOU WISH ' WRITE $(6, *)$. TO DECAY INVENTORY OUT TO: WRITE $(6, *) \cdot \begin{array}{llll}\text { (a) } 75 \text { yrs. } & \text { (b) } 100 \text { yrs. } & \text { (c) } 500 \text { yrs. POST0392 }\end{array}$

WRITE $(6, *) \cdot$ (d) $1000 \mathrm{yrs} . \quad$ (e) $5000 \mathrm{yrs}$ (f) $10,000 \mathrm{yrs.}$ ( POST0393 WRITE $(6, *)$ READ 2000, choice 2000 . FORMAT (al)

(f) 10,000 yrs. ' POST0393

POST0394

POST0396

POST0397

POST0398

POST0399

POST0400

POST0401 WILI BE NAMED SUCH THAT THE LAST LETTER OF THE FILENAME INDICATES C HOW FAR INTO THE FUTURE THE BASE YEAR DATA HAS BEEN DECAYED. FOR

C EXAMPLE, ornlc95a.u5 IS THE NAME FOR ORNL Contact handled waste C 1995 INVENTORY TO BE DECAYED OUT 75 YEARS.

C

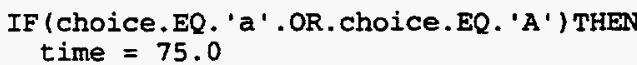
time $=75.0$

POST 0402

POST0403

POST0404

POST 0405

POST0406

POST 0407

POST0408

POST0409

POST0410

POST0411

POST0 112

POST0413

POST0414

POST0415

POST0416

POST 0417

POST0418

POST0419

POST 0420

POST0421

POST0422

POST0423

POST0424

RETURN

POST 0425

CCCCCCCCCCCCCCCCCCCCCCCCCCCCCCCCCCCCCCCCCCCCCCCCCCCCCCCCCCCCCCCCCCCCCCCCPOST0426 $\begin{array}{ll}\text { SUBROUTINE BLANKS (names, tname) } & \text { POST0427 }\end{array}$

$\begin{array}{ll}\text { C } & \text { POST0428 } \\ \text { C jennifer liscum-Dowell }-936411 / 30 / 95 & \text { POST0429 }\end{array}$

CCCCCCCCCCCCCCCCCCCCCCCCCCCCCCCCCCCCCCCCCCCCCCCCCCCCCCCCCCCCCCCCCCCCCCCCPOST 0430

$C$ This subroutine removes all blanks from isotope names as CPOST0431

C written in ORIGEN2 output in order to use subroutine CONV $\quad$ CPOST0432

C to convert from curies in ORIGEN2 output file to grams and CPOST0433

C generate a new ORIGEN2 input file. $\quad$ CPOST0434

CCCCCCCCCCCCCCCCCCCCCCCCCCCCCCCCCCCCCCCCCCCCCCCCCCCCCCCCCCCCCCCCCCCCCCCCPOST0435

IMPLICIT REAL $(a-h, 0-z)$, INTEGER $(i-n) \quad$ POST0436

CHARACTER let $(6) \star 1$, names*6, tname*6, pos $(6) \star 1,1$ pos $* 6 \quad$ POST0437

INTEGER blank, cnt, space, sp, $k$

$\mathrm{C}$

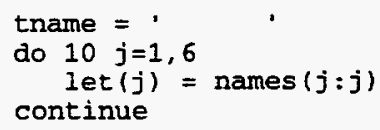

POST0442

POST0443

POST0444

POST0445

POST0446

POST0447

space $=0$

nent $=0$

cnt $=0$

blank =

do $15 j=1,6$

if (let $(j)$.eq. ' ') blank=blank+1

15 continue

POST0448

POST0449

POST0 450

POST0451

POST0452

POST0453

do $20 j=1,6$

if (let $(j)$.eq. ') then $\operatorname{pos}(j)=1-1$ 


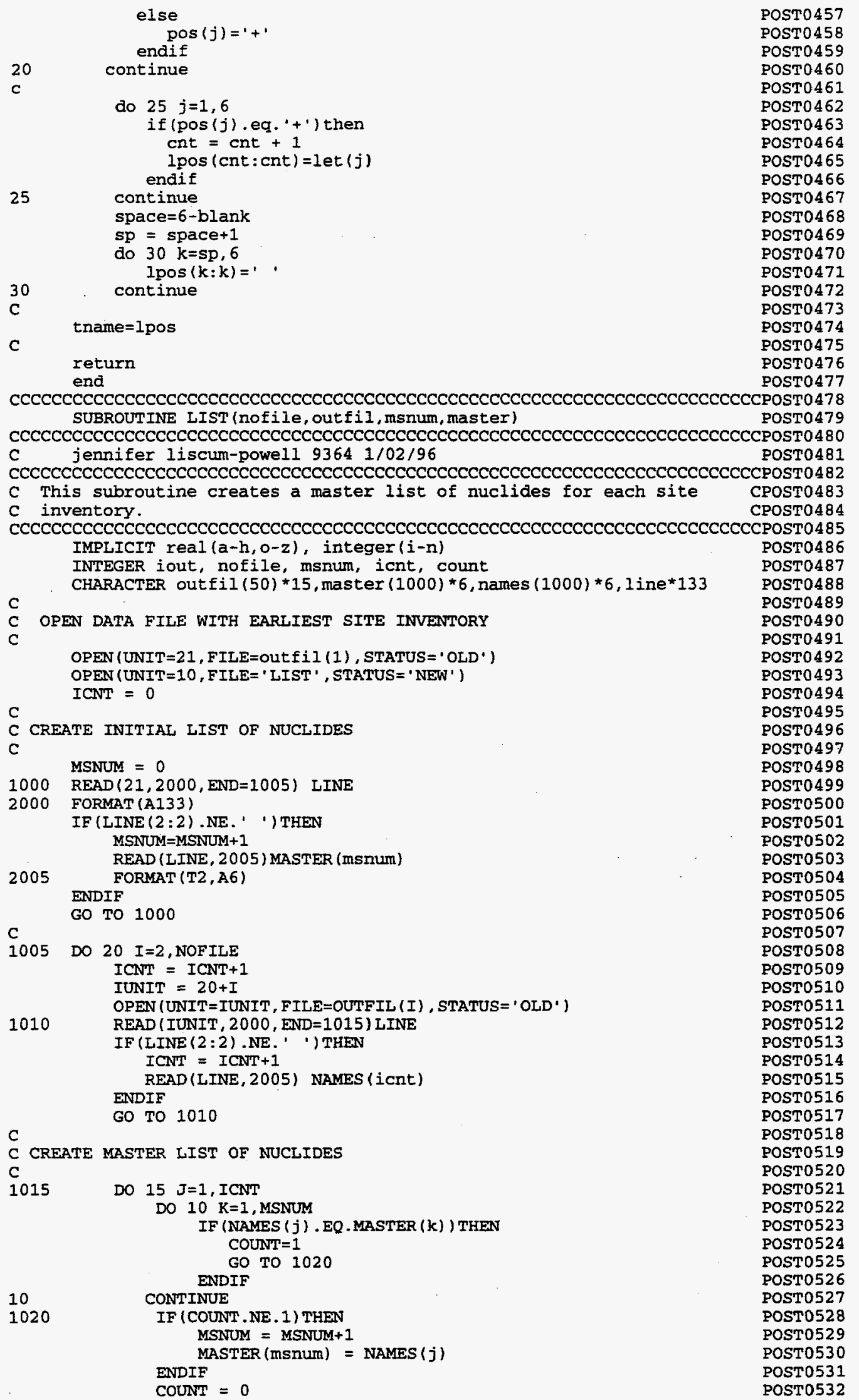


ICNT $=0$

20 CONTINUE

C DO $22 I=1$, MSNUM WRITE $(10,5000)$ MASTER (I)

5000 FORMAT (T2, A6)

22 CONTINUE

C

CLOSE (UNIT $=10$ )

DO $25 i=1$, nofile

iout $=20+i$

25

CLOSE (unit $=$ iout)

CONTINUE

$$
\text { RETURN }
$$

END

POST0 533

POST0534

POSTO535

POST0536

POST0537

POST0538

POST0539

POST0540

POST0541

POST0542

POST0543

POST0544

POST0545

POST0546

POST 0547

POST0548

POST0549

POST0550

CCCCCCCCCCCCCCCCCCCCCCCCCCCCCCCCCCCCCCCCCCCCCCCCCCCCCCCCCCCCCCCCCPOST0551 SUBROUTINE ABC (msnum, master)

POSTO552

C

jennifer 1iscum-powell - $936412 / 18 / 95$

POST0553

POST0554

CCCCCCCCCCCCCCCCCCCCCCCCCCCCCCCCCCCCCCCCCCCCCCCCCCCCCCCCCCCCCCCCCCCCCPOST0 55

C This subroutine alphabetizes the master list of nuclides prior cPosT0556

$C$ to calculating totals at end of base year.

CPOST0557

CCCCCCCCCCCCCCCCCCCCCCCCCCCCCCCCCCCCCCCCCCCCCCCCCCCCCCCCCCCCCCCCCCCCPOSTO558 IMPLICIT real $(a-n, 0-z)$, integer $(i-n) \quad$ POSTO559 INTEGER msnum

C CHARACTER master $(1000) * 6$, TEMP*6

C do $15 j=1$, msnum-1

do $10 i=1$, msnum- 1

if (master (i) . ge . master $(i+1)$ ) then TEMP = master $(i)$

master $(i)=\operatorname{master}(i+1)$ master $(i+1)=$ TEMP

10 continu endif

C 15 continue

C

return

end

POST0560

POST0561

POST0562

POST0 563

POST0 564

POST0565

POST0566

POST0 567

POST0568

POST0 569

POST0570

POST0571

POST0 572

POST0573

POST0574

POST0575

POST0576

CCCCCCCCCCCCCCCCCCCCCCCCCCCCCCCCCCCCCCCCCCCCCCCCCCCCCCCCCCCCCCCCCCCCCCPOSTO577 SUBROUTINE ABCIIN (lcnt, msIine, sorted)

POST0578

POST0579

C

jennifer 1iscum-powel1 - $93641 / 04 / 96$

POST0580

CCCCCCCCCCCCCCCCCCCCCCCCCCCCCCCCCCCCCCCCCCCCCCCCCCCCCCCCCCCCCCCCCCCCCCPOST0581

C This subroutine alphabetizes the master list of nuclides tracking cPostos82

$C$ entire lines of data containing inventory decayed into the future. CPosTo583

CCCCCCCCCCCCCCCCCCCCCCCCCCCCCCCCCCCCCCCCCCCCCCCCCCCCCCCCCCCCCCCCCCCCCCPOST0584

IMPLICIT real $(a-h, 0-z)$, integer $(i-n) \quad$ P0ST0585

INTEGER lcnt

CHARACTER msline $(1000) \star 133$, sorted $(1000) \star 133$, TEMP $* 133$,

C name $(1000) * 6$, trame* 6

DO $20 j=1,1$ cnt -1

C

DO $15 i=1$, $1 \mathrm{cnt}-1$ READ (msline (i), 2000) name (i)

$2000 \quad$ FORMAT (T2, A6)

CALL BLANKS (name (i), tname)

name $(i)=$ tname

c

READ (msline $(i+1), 2000$ ) name $(i+1)$

CALL BLANKS (name $(i+1)$, tname)

name $(i+1)=$ tname

IF (name (i) . ge name ( $i+1)$ ) THEN

c write $(6, \star)$ name $(i),+\&$ name $(i+1)$

TEMP = msline (i)

msline $(i)=$ msline $(i+1)$

Iine $(i+1)=$ TEMP

endif

15 continue

c

POST0586

POST0587

POST0588

POST0589

POST0590

POST0591

POST0592

POST0593

POST0594

POST0595

POST0596

POST0597

POST0598

POST0599

POST0600

POST0601

POST0602

POST0603

POST0604

POST0605

POST0606

POST0607

POST0608 


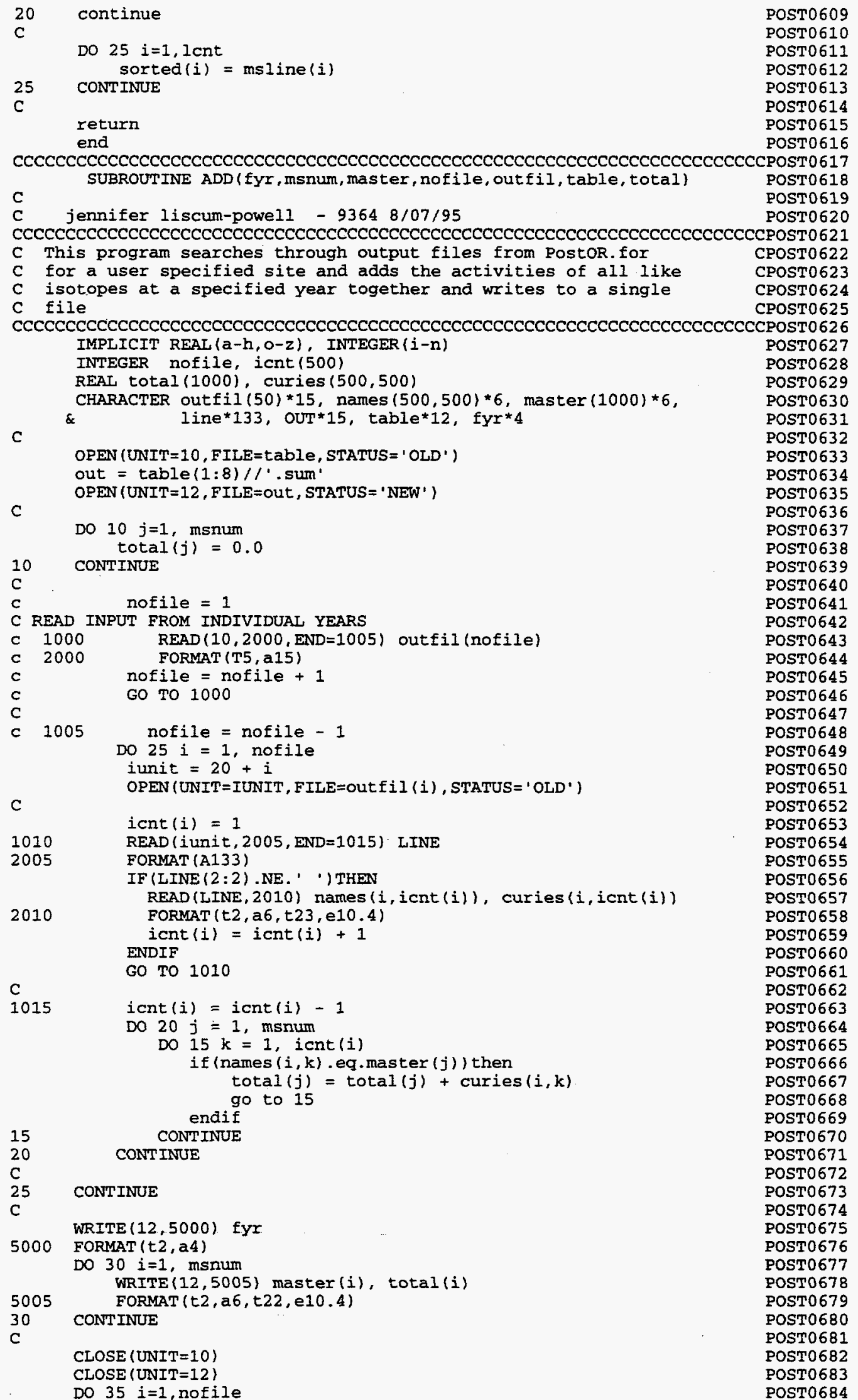




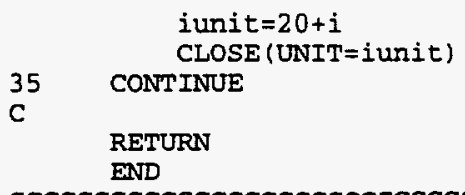

POST0685

POST0686

POST0687

POST0688

POST0689

POST0690

$\operatorname{CCCCCCCCCCCCCCCCCCCCCCCCCCCCCC~}$ SUBROUTINE WIPP (BIDINP) CCCCCCCCCCCCCCCCCCCCCCCCCCCCCCCCCCCCCCCCCCCCCCCCCCCCCCCCCCCCCCCCCCCCCCCC $\mathrm{C}$

C jennifer liscum-powell $93641 / 04 / 96$

(2) CCCCCCCCCCCCCCCCCCCCCCCCCCCCCCCCCCCCCCCCCCCCCCCCCCCCCCCCCCCCCCCCCCCCCCCCPOST0696

$C$ This subroutine can be used to sort inventories in *.sum files CPOST0697

C generated by postoRg. for to include those listed in Appendix E CPOST0698

$C$ of the WIPP Baseline Inventory Report revision 1 (February 1995) CPOST0699 CCCCCCCCCCCCCCCCCCCCCCCCCCCCCCCCCCCCCCCCCCCCCCCCCCCCCCCCCCCCCCCCCCCCCCCCPOST0700 IMPLICIT REAL $(a-h, 0-z), \operatorname{INTEGER}(i-n)$ INTEGER icnt

REAL sumed $(500)$, curies (500)

CHARACTER name $(500) * 6$, iname $(500) * 6$, type $* 2$, input* 15 , output*15, line*133, BIDINP*15

C OPEN DATA FILES

input $=$ BIDINP

output $=$ input $(1: 8) / /$ '. bid

C

WRITE $\left(6,{ }^{*}\right)$ ' WIPP BIR Appendix E Nuclide List File: ', output

OPEN (UNIT $=10$, FILE $=$ input, STATUS $=$ ' OLD' $)$

OPEN (UNIT =15, FILE=outPut, STATUS= ' NEW' )

C

WRITE $(6, *)$ INPUT $(5: 5)$

IF (input $(5: 5)$. EQ. ' $C$ ' . OR. input $(5: 5)$. eq. 'C') THEN type $=$ ' ch'

ELSEIF (input (5:5) EO. 'b' . OR. input (5:5) . eq. 'B') THEN type $=$ ' bu '

ELSEIF (input ( $5: 5)$.EQ. ' $r$ ' . OR . input $(5: 5)$. eq. ' $R$ ') THEN type $=$ ' $r$ '

ENDIF

WRITE $(6, \bullet)$ TYPE

C

READ NAMES AND CONCENTRATIONS FROM INPUT FILE

iskip $=0$

icnt $=0$

1000 READ $(10,2005$, END $=1010)$ line

2005 FORMAT (a80)

iskip = iskip +1

IF (iskip.GT.I) THEN

icnt $=$ icnt +1

READ (1ine, 2010) iname(icnt), curies (icnt)

2010 FORMAT (T2, A6, T22, E10,4)

C ENDIF WRITE $(6,2010)$ INAME (ICNT), CURIES (ICNT)

GO TO 1000

1010 CALL NAMES (type, name)

IF (type.EQ. 'ch' .OR. type.EQ. 'bu') THEN

DO $10 I=1,136$

$\operatorname{SUMED}(I)=0$

10 CONTINUE

DO $15 k=1,136$

IF (iname (j) .EQ.name (k)) THEN

sumed $(k)=$ curies $(j)$

$$
\text { ENDIF }
$$

15 CONTINUE

20 CONTINUE

MEITE TO OUTPUT FILE

DO $25 j \cdot=1,136$

WRITE $(15,5005)$ NAME $(j), \operatorname{SUMED}(j)$
POST0701

POST0702

POST0703

POST0704

POST0705

POST0706

POST0707

POST0708

POST0709

POST0710

POST0711

POST0712

POST0713

POST0714

POST0715

POST0716

POST0717

POST0718

POST0719

POST0720

POST0721

POST0722

POST 0723

POST0724

POST0725

POST0726

POST0727

POST0728

POST0729

POST0730

POST0731

POST0732

POST0733

POST0734

POST0735

POST0736

POST0737

POST0738

POST0739

POST0740

POST0741

POST0742

POST0743

POST0744

POST0745

POST0746

POST0747

POST0748

POST0749

POST0750

POST0751

POST0752

POST0753

POST0754

POST0755

POST0756

POST0757

POST0758

POST0759

POST0760 


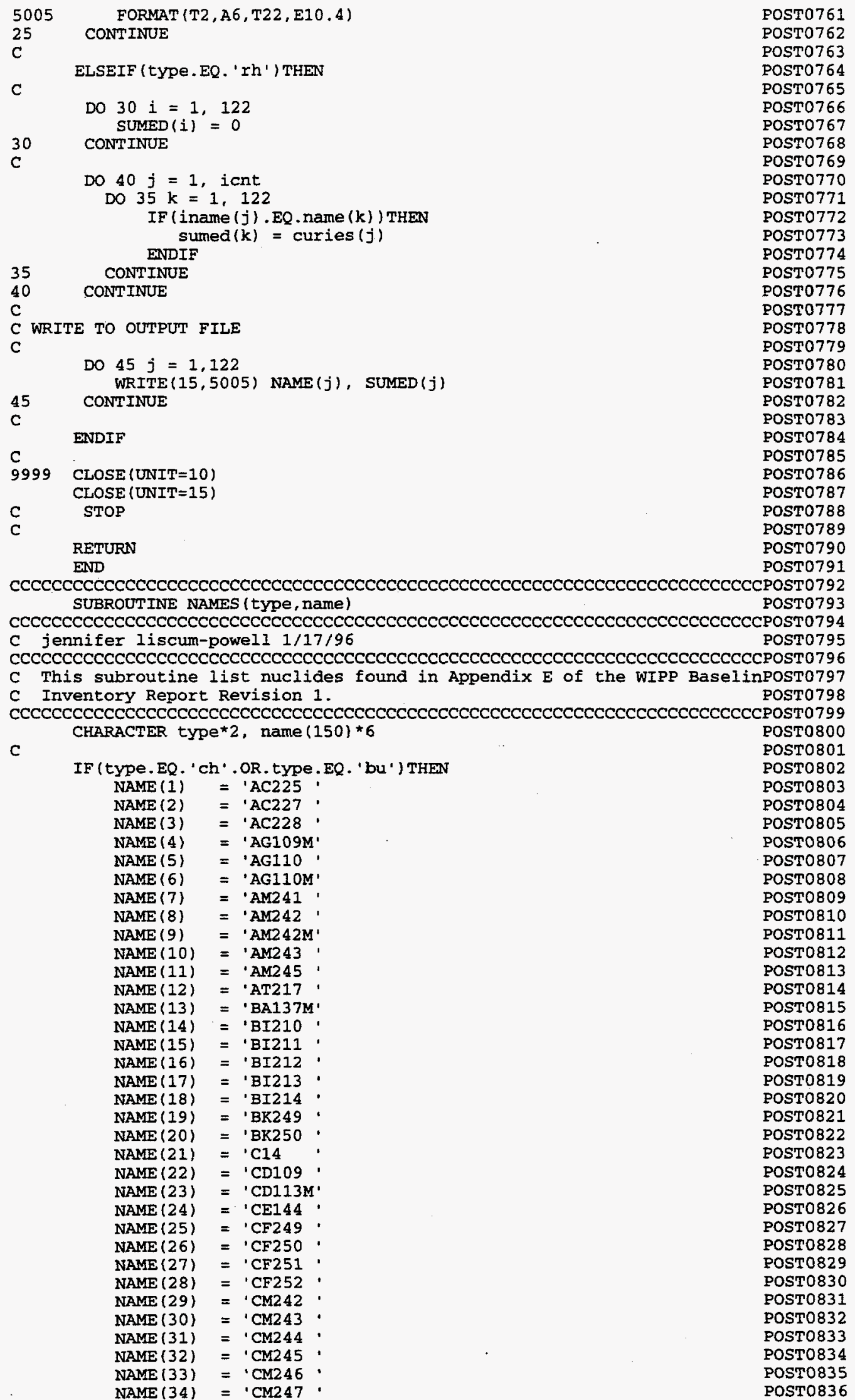




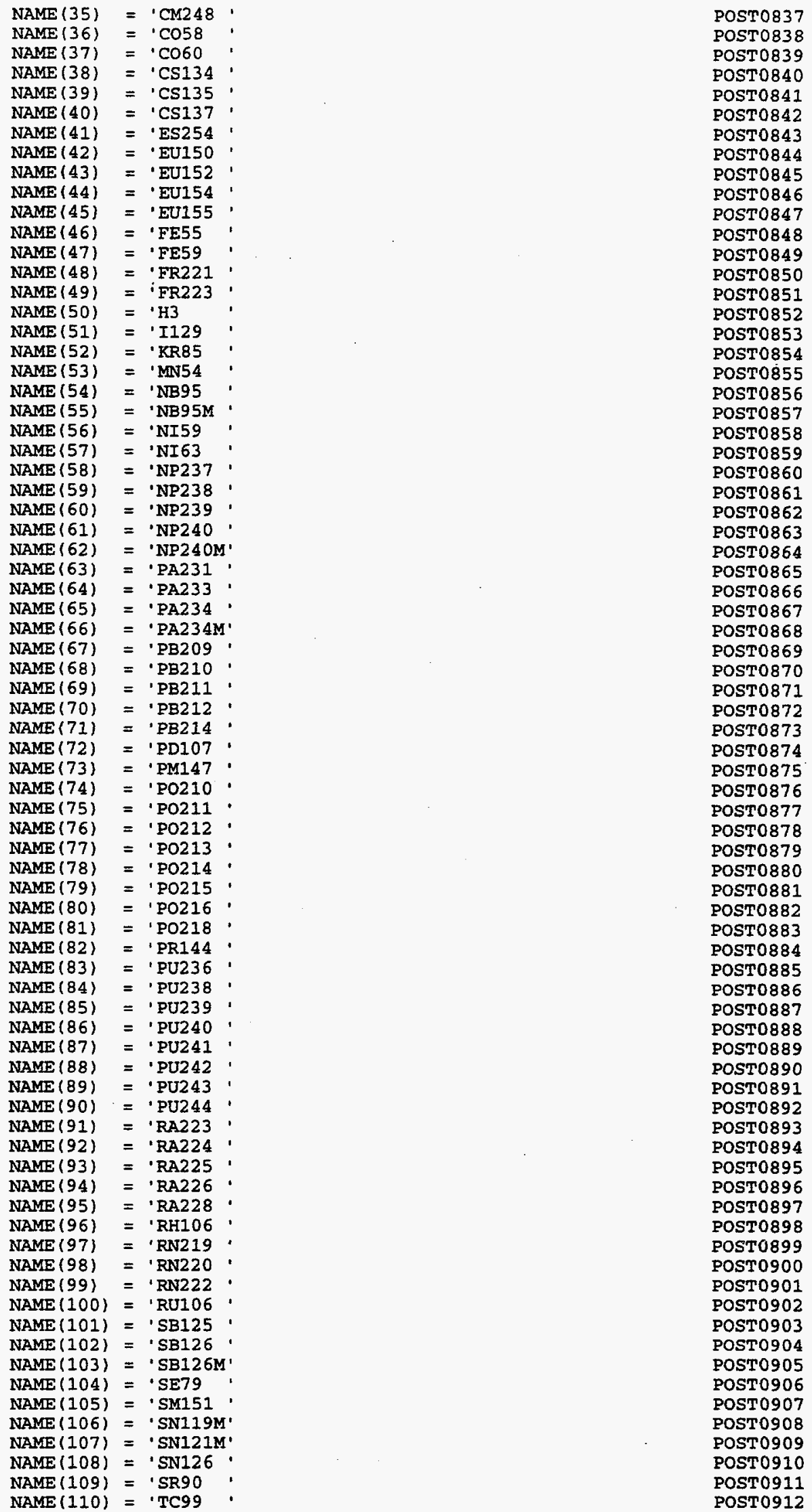




\begin{tabular}{|c|c|}
\hline NAME (111) & $=$ \\
\hline $\operatorname{NAME}(112)$ & TE127 \\
\hline NAME (113) & ' TE127 \\
\hline NAME (114) & $=$ 'TH227 \\
\hline NAME (115) & $=\mathrm{TH} 228$ \\
\hline $\operatorname{NAME}(116)$ & $=\mathrm{TH} 229$ \\
\hline $\operatorname{AME}(117)$ & $=\quad T H 230$ \\
\hline AME (118) & $=\quad \mathrm{TH} 231$ \\
\hline NAME (119) & $=\mathrm{TH} 232$ \\
\hline NAME (120) & $={ }^{\prime} \mathrm{TH} 234$ \\
\hline NAME (121) & $=\operatorname{TL} 207$ \\
\hline NAME $(122)$ & $=T L 208$ \\
\hline$M E(123)$ & $=T L 209$ \\
\hline $\operatorname{ME}(124)$ & $=T L 210$ \\
\hline (125) & $=\cdot \mathrm{U} 232$ \\
\hline $\operatorname{NAME}(126)$ & $=\cdot \mathrm{U} 233$ \\
\hline NA & $=\mathrm{U} 234$ \\
\hline (128) & $=\cdot \mathrm{U} 235$ \\
\hline & $=\cdot \mathrm{U} 236$ \\
\hline NA & $=\mathrm{U} 237$ \\
\hline (131) & $=\mathrm{U} 238$ \\
\hline 2) & $=' U 240$ \\
\hline & $=' Y 90$ \\
\hline NA & $=$ ZN95 \\
\hline & $=$ ZR93 \\
\hline NAI & $=\mathrm{ZR} 95$ \\
\hline & Q. $\left.\cdot \mathrm{rh}^{\prime}\right) \mathrm{I}$ \\
\hline & \\
\hline $\mathrm{NA}$ & $=A C 227$ \\
\hline & $=A C 228$ \\
\hline & $=' A G 110$ \\
\hline & $=$ AG110 \\
\hline$N$ & ' AM241 \\
\hline & $=$ AM243 \\
\hline & $=\cdot A M 245$ \\
\hline & $=\cdot \operatorname{AT} 217$ \\
\hline & $={ }^{\prime} \mathrm{BA} 137 \mathrm{~F}$ \\
\hline & $=' B I 210$ \\
\hline & $=\cdot B I 211$ \\
\hline & $=' B I 212$ \\
\hline & BI213 \\
\hline & $=$ \\
\hline & $=\cdot B K 249$ \\
\hline & $={ }^{\prime} \mathrm{C} 14$ \\
\hline & $={ }^{\circ} \mathrm{CD} 113 \mathrm{l}$ \\
\hline & $={ }^{\prime} C E 144$ \\
\hline & $={ }^{\circ} \mathrm{CF} 249$ \\
\hline & $=\cdot \mathrm{CF} 250$ \\
\hline & 52 \\
\hline & $=\cdot \mathrm{C}$ \\
\hline & $=\cdot \mathrm{CM}$ \\
\hline & $=\cdot \mathrm{CM}$ \\
\hline & $=\mathrm{CM}^{2}$ \\
\hline & $=\cdot \mathrm{CM} 248$ \\
\hline & ' $\mathrm{CO}$ \\
\hline & . cos \\
\hline & $={ }^{\prime}$ CR51 \\
\hline & $=\cdot \operatorname{cs} 134$ \\
\hline & $\cdot \mathrm{cs}$ \\
\hline & 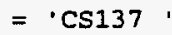 \\
\hline & ' EU \\
\hline & $=\cdot \mathrm{EU}]$ \\
\hline & \\
\hline & 'FE5 \\
\hline & ' FE5 \\
\hline & 1 \\
\hline$=0$ & $=\quad F R 2$ \\
\hline & ' $\mathrm{H} 3$ \\
\hline & ' KR8 \\
\hline & $=$ MN54 \\
\hline & $=$ 'NB9 \\
\hline (45) & $=' \mathrm{NB} 95 \mathrm{M}$ \\
\hline NA & $=\operatorname{NI} 63$ \\
\hline NA & NP2 \\
\hline 100 & NP239 \\
\hline & \\
\hline
\end{tabular}

POSTO913

POSTO914

POST0915

POST0916

POST0917

POSTO918

POST0919

POST0920

POST0921

POST0922

POST0923

POST0924

POST0925

POST0926

POST0927

POST0928

POST0929

POST0930

POST0931

POST0932

POST0933

POST0934

POST0935

POST0936

POST0937

POST0938

POST0939

POST0940

POST0941

POST0942

POSTO943

POST0944

POSTO945

POST094 6

POST0947

POST0948

POST0949

POST0950

POST0951

POST0952

POST0953

POST0954

POST0955

POST0956

POST0957

POST0958

POST0959

POST0960

POST0961

POST0962

POST0963

POST0964

POST0965

POST0966

POST0967

POST0968

POST0969

POST0970

POST0971

POST0972

POST0973

POST0974

POST0975

POST0976

POST0977

POST0978

POST0979

POST0980

POST0981

POST0982

POST0983

POST0984

POST0985

POST0986

POST0987

POST0988 


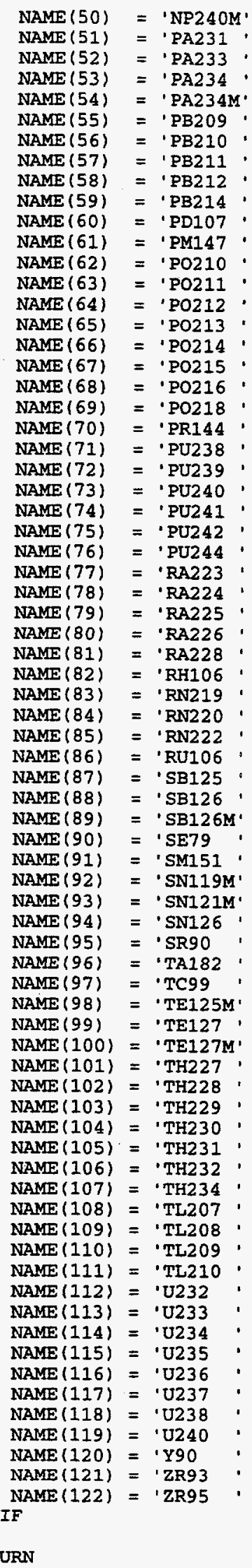

POST0989

POST0990

POST0991

POST0992

POST0993

POST0994

POST0995

POST0996

POST0997

POST0998

POST0999

POST 1000

POST 1001

POST 1002

POST 1003

POST1004

POST 1005

POST 1006

POST 1007

POST1008

POST 1009

POST1010

POST1011

POST 1012

POST1013

POST1014

POST 1015

POST 1016

POST 1017

POST1018

POST1019

POST1020

POST1021

POST 1022

POST1023

POST1024

POST 1025

POST 1026

POST 1027

POST 1028

POST1029

POST 1030

POST 1031

POST1032

POST 1033

POST 1034

POST 1035

POST 1036

POST1037

POST1038

POST1039

POST 1040

POST1041

POST 1042

POST1043

POST 1044

POST1045

POST 1046

POST 1047

POST1048

POST 1049

POST1050

POST1051

POST 1052

POST 1053

POST1054

POST 1055

POST 1056

POST 1057

POST 1058

POST 1059

POST 1060

POST1061

POST1062

POST 1063

POST 1064 
END

CCCCCCCCCCCCCCCCCCCCCCCCCCCCCCCCCCCCCCCCCCCCCCCCCCCCCCCCCCCCCCCCCCCCCCCCPOST1066

SUBROUTINE FINPUT (time, tstep, input)

POST1067

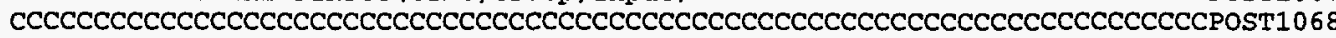
$\mathrm{C}$

C jennifer 1iscum-powell $93641 / 04 / 96$

POST 1069

CCCCCCCCCCCCCCCCCCCCCCCCCCCCCCCCCCCCCCCCCCCCCCCCCCCCCCCCCCCCCCCCCCCCCCCCPOST1071

C This subroutine generates an ORIGEN2 input file to decay inventory CPOST1072

$C$ into the future as specified by the user.

CPOST1073

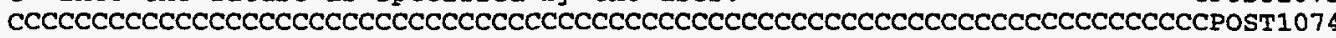
IMPLICIT REAL $(a-h, 0-z)$, INTEGER $(i-n)$

REAL curies $(100,500)$, grams $(1,500)$, time, tsteo

INTEGER ycnt, isonum, var1 $(500), \operatorname{var} 2(500), \operatorname{var} 3(500)$, lib $(100,500)$, yr out, hed $0: 11)$ dectim

CHARACTER input $* 15$, outfil*15, line*133, choice*1,

$\delta$ ucnam $(100,500) * 6$, nucid $(100,500) * 6$, fyr ${ }^{*} 4$

IF (tstep.EQ.7.50) THEN

choice $=$ ' $a$ '

ELSEIF (tstep.EQ.10.0) THEN

choice $=$ ' $b$ '

ELSEIF (tstep.EQ.50.0) THEN

choice $=$ ' $c$ '

ELSEIF (ts tep.EQ.100.0) THEN choice $=$ ' $d$ '

ELSEIF (tstep.EQ.500.0) THEN choice $=$ ' $e$ '

ELSEIF (tstep.EQ.1000) THEN

choice $=$. $f$ '

ENDIF

outfil = input $(1: 5) / /$ input $(7: 8) / /$ choice $/ / 1$, u5

WRITE $(6, \star)$ ' ORIGEN2 input filename $=$ ', outfil

$\mathrm{C}$

OPEN INPUT AND OUTPUT FILES

OPEN (UNIT $=12$, FILE $=$ input , STATUS $=$ ' OLD' )

OPEN (UNIT $=15$, FILE= out fil, STATUS = ' NEW'

C READ INPUT DATA FROM FILE

ycnt $=0$

icnt $=1$

istep $=0$

c

$1000 \operatorname{READ}(12,2000, \operatorname{END}=1005)$ Iine

2000 FORMAT (a133)

istep $=$ istep +1

IF (line ( $2: 3)$.EQ. ' 19 '. OR. I ine (2:2) .EQ. '2') THEN

yont $=y$ cnt +1

isonum $=0$

READ (LINE, 2005) yr

2005 FORMAT (t2, i4)

GO TO 1000

ENDIF

C

READ ISOTOPES AND ACTIVITIES IN INVENTORY FOR PRESENT YEAR AND SITE

IF (istep.GT.2) THEN

isonum $=$ isonum +1

READ(1ine, 2010) nucnam(1, isonum), curies (1, isonum)

2010 FORMAT (T2, A6, T22, e10.4)

IF (curies ( 1 , isonum) .EQ.0) THEN isonum $=$ isonum -1

ENDIF

GO TO 1000

C ENDIF

1005 out $=15$

C

C WRITE TO ORIGEN2 PC INPUT FILE

WRITE (out, 5000)

5000 FORMAT $\left(2(2 x, 1-1,1), 2 x, 1^{\prime}-1 '\right)$

C

WRITE (out, 5005) time

POST1076

POST1077

POST1078

POST1079

POST1080

POST1081

POST1082

POST1083

POST1084

POST1085

POST1086

POST 1087

POST1088

POST1089

POST 1090

POST1091

POST1092

POST1093

POST1094

POST1095

POST1096

POST1097

POST1098

POST1099

POST 1100

POST 1101

POST 1102

POST 1103

POST1104

POST 1105

POST 1106

POST 1107

POST 1108

POST1109

POST1110

POST1111

POST1112

POST 1113

POST1114

POST1115

POST 1116

POST1117

POST 1118

POST1119

POST 1120

POST 1121

POST1122

POST1123

POST1124

POST1125

POST 1126

POST 1127

POST 1128

POST 1129

POST 1130

POST1131

POST1132

POST 1133

POST 1134

POST 1135

POST 1136

POST1137

POST 1138

POST1139

POST1140 
5005 FORMAT (2x, 'BAS', 4x, 'WIPP BASELINE INVENTORY DATA DECAY OUT' ', \& $\quad$ 7.1,' Yrs')

WRITE (out, 5010) input $(1: 8)$

5010 FORMAT ( $2 x$, 'TIT', $4 x, a 8$ )

WRITE (out, 5015)

5015 FORMAT $\left(2 x\right.$, 'LIP', $\left.4 x, 3\left({ }^{\prime} 0 \cdot, 2 x\right)\right)$

WRITE (out, 5020)

5020 FORMAT $\left(2 x,{ }^{\prime}\right.$ IIB', 3x, $\left.0^{\prime}, 2 x, 1^{\prime}, 2 x, 2^{\prime}, 2 x, 3^{\prime}, 2 x, 3()^{\prime}, 2 x\right)$, $+$ $\left.\cdot 9 \cdot, 2 x, \cdot 3 \cdot, 2 x, \cdot 0^{\circ}, 2 x, 1^{\prime}, 2 x, \cdot 1 '\right)$

WRITE (out, 5025)

5025 FORMAT (2x, 'PHO', 4x, '101', 2x, '102', 2x, '103', 2x, '10')

C

$j=1$

WRITE (out, 5030) j

5030 FORMAT ( $2 x$, 'INP', $4 x, 14,2 x, 11 \cdot, 2 x, 2(\cdot-1 ', 2 x), 2(\cdot 1 \cdot, 2 x))$

C

WRITE (out; 5035) yr

5035 FORMAT (2x, 'RDA', 4x, 'BASE YEAR IS ', i4,' $(T=0)$ ') WRITE (out, 5040)

5040 FORMAT ( $2 x$, 'RDA', $4 x$, 'ALL OTHER HEADINGS ARE IN YEARS')

dectim $=0$

hed $(0)=0$

DO $10 i=1,10$

$j=i+1$

dectim $=$ dectim + tstep

hed $(i)=\operatorname{dectim}$

IF (i.EQ.1)THEN

WRITE (out, 5045) dectim, $i, j$

5045 FORMAT (2x,'DEC', $4 x, i 7,2 x, i 4,2 x, i 4,1 x$,

ELSE

$$
\text { ' } \left.5 \text {, } 1 \mathrm{x}, \mathbf{1}^{\prime}\right)
$$

WRITE(out, 5050) dectim, i, j
$5050+\quad$ FORMAT $(2 x$, DEC', $4 x, i 7,2 x, i 4,2 x, i 4,1 x$,

ENDIF

$\begin{array}{ll}10 & \text { CONTINUE } \\ \mathrm{C} & \\ \mathrm{C} & \text { WRITE TITLES FOR OUTPUT VECTORS }\end{array}$

C WRITE TITLES FOR

5055 FORMAT (2x, 'RDA', 4x, 'ASSIGN TITLES TO OUTPUT VECTORS')

DO $15 i=1,11$

$j=i-1$

IF (j.EQ.0) THEN

WRITE (out, 5060$) i$, hed (j)

5060 FORMAT $(2 x, ' H E D ', 4 x, i 4,2 x, \cdot t=\cdot, i 5)$

ELSEIF (j.GT.0) THEN

WRITE (out, 5065) $i$, hed (j)

5065 FORMAT ( $2 x$, 'HED' $, 4 x, 14,2 x, i 5,1 x, ' y '$ ') ENDIF

15 CONTINUE

C

WRITE OUTPUT COMMANDS

C

WRITE (out, 5070)

WRITE (out, 5075)

WRT $E$ (out, 5080)

W. $e($ out, 5085$)$

WS . (out, 5090)

WR-TE (out, 5095)

5070 FORMAT ( $2 x$, 'RDA', $4 x, \cdot$ PRINT NUCLIDE QUANTITIES IN CURIES')

5075 FORMAT $\left(2 x, '\right.$ OPTA' $\left., 3 x, ' 6 * 8{ }^{\prime}, 2 x, ' 5{ }^{\prime}, 2 x, ' 17 * 8 \cdot\right)$

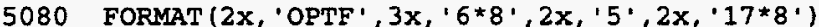

5085 FORMAT $\left(2 x,{ }^{\prime}\right.$ OPTL' $\left., 3 x, 6^{\prime} * 8^{\prime}, 2 x, ' 5^{\prime}, 2 x, \cdot 17 * 8 \cdot\right)$

5090 FOFSAT ( $2 x, \cdot$ RDA', $4 x$, 'OUTPUT COMMANDS')

5095 FOAMAT $\left(2 \mathrm{x}\right.$, 'OUT $\left.^{\prime}, 4 \mathrm{x}, \cdot 11^{\prime}, 2 \mathrm{x}, 1^{\prime}, 2 \mathrm{x}, \cdot-1^{\prime}, 2 \mathrm{x},{ }^{\prime} 0^{\prime}\right)$

$\mathrm{C}$

WRITE (out, 5100)

5100 FORMAT (2x, 'END')

C WRITE INITIAL NUCLIDE INVENTORIES TO ORIGEN2 INPUT FILE

C WRITE INITIAL NUCLIDE

$$
\begin{aligned}
& \text { num }=\text { isonum } \\
& \text { lib1 }=0 \\
& \text { lib2 }=0
\end{aligned}
$$

POST1141

POST 1142

POST1143

POST 1144

POST1145

POST1146

POST1147

POST1148

POST 1149

POST 1150

POST1151

POST1152

POST1153

POST1154

POSTI155

POST1156

POST1157

POST1158

POST1159

POST 1160

POST1161

POST1162

POST1163

POST1164

POST1165

POST 1166

POST 1167

POST 1168

POST1169

POST1170

POST1171

POST 1172

POST1173

POST1174

POST1175

PosT1176

POST1177

POST 1178

POST1179

POST 1180

POST1181

POST1182

POST1183

POSTI184

POST1185

POST1186

POST1187

POST1188

POST1189

POST1190

POST1191

POST1192

POST1193

POST1194

POST1195

POST1196

POST1197

POST1198

POST1199

POST 1200

POST1201

POST1202

POST1203

POST1204

POST 1205

POST1206

POST 1207

POST1208

POST1209

POST 1210

POST1211

POST 1212

POST1213

POST1214

POST 1215

POST1216 
$1 i b 3=0$

lcnt $=0$

POST 1217

POST 1218

$c$

C CONVERT INITIAL NUCLIDE CONCENTRATIONS FROM CURIES TO GRAMS AND C ASSIGN ORIGEN2 SPECIFIC NUCLIDE IDENTIFIER

C

DO $20 j=1$, num

$\operatorname{CALL} \operatorname{CONV}(\operatorname{nucnam}(1, j), \operatorname{curies}(1, j), \operatorname{nucid}(1, j)$,

20 CONTINUE $\operatorname{grams}(1, j), \operatorname{lib}(1, j))$

DO $25 k=1$, num

$\operatorname{IF}(1 i b(1, k)$.EQ.1) THEN

$1 \mathrm{ib} 1=1 \mathrm{ib} 1+1$

$\operatorname{var} 1(1 i b 1)=k$

ELSEIF ( $1 i b(1, k)$. EQ . 2$)$ THEN

$1 i b 2=1 i b 2+1$

$\operatorname{var} 2(1 \mathrm{ib2})=k$

ELSEIF $(1 j b(1, k)$.EQ.3) THEN

$1 i b 3=1 i b 3+1$

$\operatorname{var} 3(1 i b 3)=k$

25 CONTINUE

NDIF

C FOR PRESENT YEAR, FROM IIBRARY 1 - ACTIVATION PRODUCTS

c

IF ( 1 ibl. LE. 3 ) THEN
num $=1$ ibl

IF (Iib1.EO.1) THEN

WRITE (out, 5105) (nucid(1, $\operatorname{var} 1(L)), \operatorname{grams}(1, \operatorname{var} 1(L))$, $L=1,1$ ib 1 )

5105

FORMAT $(2 x, 11,5 x, A 7,2 x$

ELSEIF (1ib1. EQ.2) THEN

WRITE (out.5110) (nucid(1, var1(L)), grams (1, $\operatorname{var} 1(L)$ ), $L=1,2$ )

$5110^{\&}$

$5115^{\&}$

FORMAT $\left(2 x, 1^{\prime}, 5 x, 2(a 7,2 x\right.$, e10 $\left.4,2 x), 0^{\prime}, 2 x, 0^{\prime}\right)$

ELSEIF ( I Ib1.EQ.3) THEN

WRITE (out. 5115) (nucid(1, var1(L)), grams (1, var1(L)), $\mathrm{L}=1,3$ )

FORMAT $(2 x, 11,5 x, 3(a), 2 x, e 10,4,2 x), 0 \cdot, 2 x, 0 \prime)$ ENDIF

ELSEIF ( lib1.GT . 3) THEN

ldivl $=\operatorname{int}(1 \mathrm{ib} 1 / 3)$

ldiv2 = lib1 - 3*1div1

$\mathrm{m}=0$

IF (laiv2.EO.0) THEN

$m=m+3$

IF (m. LE. IIb1) THEN

$\mathrm{n}=\mathrm{m}-2$

5120

WRITE (out, 5120 )

) (nucid (1, $\operatorname{var} I(L)), \operatorname{grams}(1, \operatorname{var} 1(L))$, $\mathrm{L}=\mathrm{n}, \mathrm{m})$

FORMAT $\left(2 x, 1^{\prime}, 5 x, 3(a 7,2 x, e 10.4,2 x), 0^{\circ}, 2 x, 0^{\prime}\right)$

GO TO 1010 ENDIF

ELSEIF (1div2.GT.0) THEN

IF (1 div1.EQ.1) THEN

WRITE (out, 5120) (nucid(1, $\operatorname{var} 1(L)), \operatorname{grams}(1, \operatorname{var} 1(L))$, $L=1,3$ )

IF ( Idiv2 . EQ . 1) THEN

WRITE (out, 5105) (nucid(1, var1 (L)), grams (1, $\operatorname{var1}(L)$ ) , $I=4,1 \mathrm{ib} 1$

ELSEIF (laiv2.EQ.2) THEN

WRITE (out, 5110) (nucid(1, var1(L)), grams (1, $\operatorname{var} 1(\mathrm{~L})$ ), $L=4,1 \mathrm{ib} 1$

ENDIF

ELSE

DO $30 \mathrm{k}=1$, ldiv 1

1015

$\mathrm{m}=\mathrm{m}+3$

lcnt $=1$ cnt +1

IF (ICnt.LE. Idiv1) THEN

$\mathrm{n}=\mathrm{m}-2$

WRITE (out, 5120) (nucid (1, $\operatorname{var1}(L)), \operatorname{grams}(1, \operatorname{var} 1(L))$

$$
\mathrm{I}=\mathrm{n}, \mathrm{m} \text { ) }
$$

GO TO 1015

ELSEIF (1cnt.GT . 1div1) THEN

$\mathrm{m}=\mathrm{m}-3$
POST1219

POST 1220

POST1221

POST 1222

POST1223

POST1224

POST1225

POST1226

POST1227

POST 1228

POST 1229

POST 1230

POST1231

POST 1232

POST 1233

POST 1234

POST 1235

POST 1236

POST 1237

POST 1238

POST 1239

POST 1240

POST1241

POST 1242

POST1243

POST1244

POST 1245

POST 1246

POST 1247

POST1248

POST 1249

POST 1250

POST1251

POST 1252

POST 1253

POST 1254

POST 1255

POST 1256

POST 1257

POST1258

POST 1259

POST1260

POST1261

POST 1262

POST 1263

POST 1264

POST 1265

POST 1266

POST 1267

POST1268

POST 1269

POST 1270

POST1271

POST1272

POST1273

POST1274

POST 1275

POST1276

POST 1277

POST 1278

POST1279

POST 1280

POST1281

POST1282

POST 1283

POST1284

POST 1285

POST 1286

POST 1287

POST 1288

POST 1289

POST1290

POST1291

POST1292 
IF (1div2.EQ.1) THEN

$\&$

$\&$

WRITE (out, 5105) (nucid(1, $\operatorname{var1}(L)), \operatorname{grams}(1, \operatorname{var} 1(L)$ ) $\mathrm{L}=\mathrm{m}, 1 \mathrm{ib} 1)$

ELSEIF (1div2, EO.2) THEN

WRITE (out, 5110) (nucid(1, $\operatorname{var} 1(L)), \operatorname{grams}(1, \operatorname{var} 1(\mathrm{~L})$ ), $\mathrm{L}=\mathrm{m}, \mathrm{lib} 1)$

$$
\begin{aligned}
& \text { ENDIF } \\
& \text { ENDIF } \\
& \text { ENDIF }
\end{aligned}
$$

C FOR PRESENT YEAR, FROM LIBRARY 2 - ACTINIDES

lcnt $=0$

IF (1 ib2 . LE. 3) THEN

num $=1$ ib2

IF ( 1 ib2 . EQ. 1) THEN

WRITE (out, 5125) (mucid(1, var2(L)), grams (1, $\operatorname{var2}(L)$ )

5125

5130

5135 FORMAT $\{2 \mathrm{x}, \cdot 2 \cdot, \mathrm{L}=1, \mathrm{x}, \mathrm{A} 7,2 \mathrm{i}, \mathrm{x} 2$

ELSEIF ( 1 ib2. EO. 2) THEN

WRITE (out, 5130) (nucid(1, var2(L)), grams (1, $\operatorname{var2}(L))$, $L=1,2)$

FORMAT $\left(2 x, ' 2^{\prime}, 5 x, 2(a 7,2 x, e 10,4,2 x), 0^{\prime}, 2 x, 0^{\prime}\right)$

ELSEIF ( 1 ib2.EQ . 3) THEN

WRITE (out, 5135) (nucid(1, $\operatorname{var} 2(L)), \operatorname{grams}(1, \operatorname{var} 2(L)$ ) , $\mathrm{L}=1,3)$

FORMAT $\left(2 \mathrm{x},{ }^{\prime} \mathrm{2}^{\prime}, 5 \mathrm{x}, 3(\mathrm{a} 7,2 \mathrm{x}, \mathrm{e} 10.4,2 \mathrm{x}), 0^{\prime}, 2 \mathrm{x}, \mathrm{\prime}^{\prime} \mathrm{\prime}^{\prime}\right)$ ENDIF

ELSEIF ( 1 ib2 . GT . 3) THEN

laivl $=\operatorname{int}(1 \mathrm{ib} 2 / 3)$

laiv2 $=1 i b 2-3 * 1$ div1

$m=0$

1020

IF (Iaiv2.EQ.0) THEN

$m=m+3$

IF (m. LE. I ib2) THEN

$\mathrm{n}=\mathrm{m}-2$

WRITE (out, 5140) (nucid (I, $\operatorname{var2}(L)$ ) , grams (1, $\operatorname{var2}(\mathrm{L})$ ), $\mathrm{L}=\mathrm{n}, \mathrm{m})$

$$
\text { FORMAT }(2 \mathrm{x}, \cdot 2 \cdot, 5 \mathrm{x}, 3 \text { (a) }
$$$$
\text { GO TO } 1020
$$

ENDIF

ELSEIF (Idiv2.GT .0) THEN

IF (Idiv1.EQ.1) THEN

WRITE (out, 5140$)$ (nucid (1, $\operatorname{var} 2(L)), \operatorname{grams}(1, \operatorname{var} 2(L)$ ), $\mathrm{L}=1,3$ )

IF (ldiv2, EO . 1) THEN

WRITE (out, 5125) (nucid (1, var2 (L)), grams (1, var2(L)), $L=4,1$ ib 2 )

ELSEIF (1div2 . EQ . 2) THEN

WRITE (out, 5130) (nucid(1, var2(L)), grams (1, var2(I)), ENDIF $\mathrm{L}=4,1 \mathrm{ib} 2$ )

ELSE

DO $35 \mathrm{k}=1$, ldiv1

1025

$\&$ $m=m+3$

lcnt $=1 \mathrm{cnt}+1$

IF (lcnt. IE. Idiv1) THEN

$\mathbf{n}=\mathrm{m}-2$

WRITE (out, $5 \pm 40)($ nucid(1, $\operatorname{var} 2(I)), \operatorname{grams}(1, \operatorname{var} 2(I))$,

GO TO 1025

ELSEIF (lcnt.GT. Idiv1) THEN

$\mathrm{m}=\mathrm{m}-3$

ENDIF

CONTINUE

$\mathbf{m}=\mathbf{m}+1$

IF (laiv2.EQ.1) THEN

WRITE (out, 5125) (nucid(1, var2 (L)), grams (1, $\operatorname{var2(L)),~}$ $\mathrm{L}=\mathrm{m}, 1 \mathrm{ib2}$ )

ELSEIF (ldiv2 . EQ.2) THEN

WRITE (out, 5130) (nucid(1, var2(L)), grams $(1, \operatorname{var} 2(L)$ ), $\mathrm{L}=\mathrm{m}, 1 \mathrm{ib2}$ )
POST 1293

POST1294

POST1295

POST1296

POST 2297

POST1298

POST1299

POST 1300

POST 1301

POST 1302

POST 1303

POST 1304

POST 1305

POST1306

POST 1307

POST 1308

POST1309

POST 1310

POST1311

POST1312

POST1313

POST1314

POST 1315

POST 1316

POST 1317

POST1318

POST1319

POST 1320

POST1321

POST 1322

POST 1323

POST1324

POST 1325

POST1326

POST1327

POST 1328

POST1329

POST 1330

POST1331

POST 1332

POST 1333

POST1334

POST 1335

POST1336

POST 1337

POST1338

POST1339

POST 1340

POST1341

POST 1342

POST1343

POST 1344

POST 1345

POST 1346

POST 1347

POST1348

POST1349

POST1350

POST1351

POST1352

POST 1353

POST1354

POST1355

POST1356

POST1357

POST1358

POST1359

POST1360

POST 1361

POST1362

POST1363

POST 1364

POST 1365

POST1366

POST 1367

POST1368 


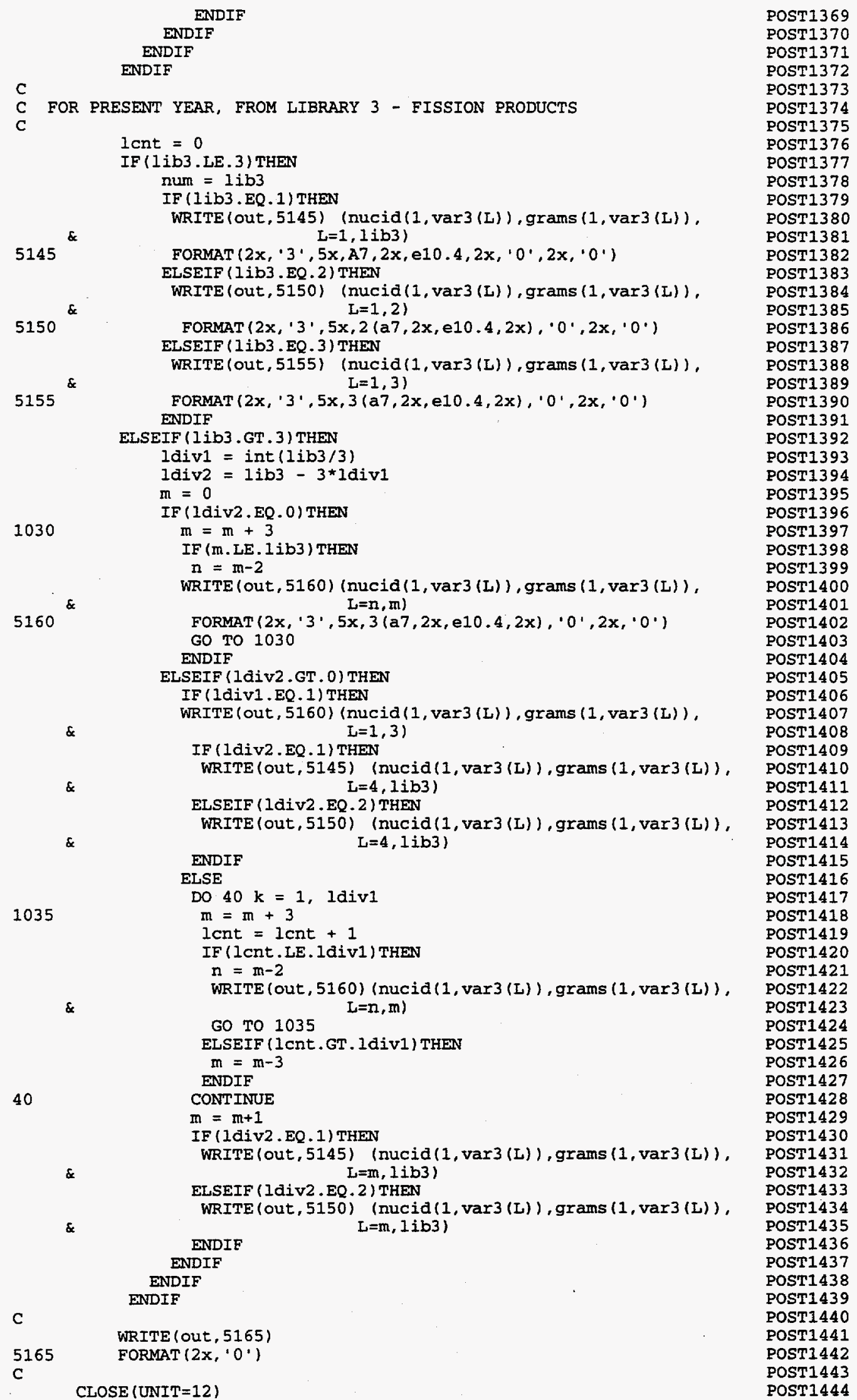


C

CLOSE (UNIT $=15$ )

POST 1445

POST1446

RETURN

END

POST 1447

POST 1448

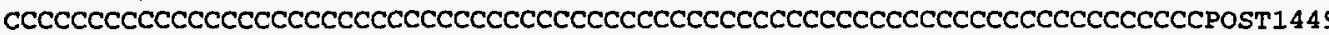
SUBROUTINE CONV (name, activ, namy, activy, nuclib) POST1450

holly trellue $67416 / 8 / 95$

POST1451

holly trellue $67416 / 8 / 95$

POST 1452

C THIS SUBROUTINE WAS WRITTEN BY HOLLY TREILUE, $6 / 8 / 95$

C MODIFIED BY J.LISCUM-POWELL 6/29/95 WITH ORIGEN2 DECAY LIBRARY HALF

C LIVES NUCLIDES ADDED 10/16/95, ADDITIONS MADE $1 / 8 / 96$

CPOST1453

CPOST 1454

CPOST 1455

CPOST 1456 CCCCCCCCCCCCCCCCCCCCCCCCCCCCCCCCCCCCCCCCCCCCCCCCCCCCCCCCCCCCCCCCCCCCCCCPOST 1457

C This subroutine converts nuclide concentrations in curies into CPOST1458

C equivalent grams of the nuclide to be used as ORIGEN2 input. CPOST1459

CCCCCCCCCCCCCCCCCCCCCCCCCCCCCCCCCCCCCCCCCCCCCCCCCCCCCCCCCCCCCCCCCCCCCCCCPOST1460

CHARACTER name ${ }^{\star} 6$, namy ${ }^{\star} 6$
POST 1461

INTEGER nuclib

REAL activ, activy, m, Thalf, sa, sal

POST1462

POST 1463

$\mathrm{C}$

C ALL VALUES FOR ATOMIC MASS NUMBERS AND HALFLIVES IN THIS FILE WERE

C TAKEN FROM THE CHART OF THE NUCLIDES.

C

$\mathrm{C}$

$$
\text { IF (name }(1: 5), E Q \cdot A C 227 \cdot \text { ) THEN }
$$

POST 1464

POST1465

POST1466

POST 1467

POST1468

POST 1469

C ATOMIC WEIGHT VALUES(M) WERE CAICULATED BY USING THE MASS EXCESS VALUEPOST1470

C FOR EACH ISOTOPE. MASS EXCESS VALUES WERE TAKEN FROM "NUCLEAR WALLET POST1471

C CARDS" BY JAGDISH TULI. JANUARY 1985. POST1472

$\mathrm{C}$

$$
\mathrm{m}=227.0+25.849 / 931.5016
$$

$\mathrm{C}$

C FOR CONFORMITY, THE HALF LIVES ARE CONVERTED TO SECONDS FOR

C EVERY ISOTOPE. AC227's HALF LIFE IS 21.77 YEARS. AIL VALUES FOR

C HALFLIVES IN THIS PROGRAM COME FROM THE ORIGEN2 DECAY LIBRARY

C

Thalf $=6.871 \mathrm{e} 08$

nucli $b=2$

namy $(1: 6)=' 892270$ '

ELSETF (name $(1: 5)$. EO. AC225') THEN

$M=225.0+21.626 / 931.5016$

Thalf $=8.640 \mathrm{e} 05$

nuclib $=2$

namy $(1: 6)=\cdot 892250$ '

ELSEIF (name (1:5). EQ. 'AC228') THEN

$\mathrm{m}=228.0+28.890 / 931.5016$

Thalf $=2.207 \mathrm{e} 04$

nuclib $=2$

$\operatorname{namy}(1: 6)=\cdot 892280^{\circ}$

ELSEIF (name (1:5). EQ.'AG110') THEN

if (name $(6: 6)$. EO. ' $M$ ') THEN

$\mathrm{m}=110.0-87.341 / 931.5016$

Thalf $=2.159 \mathrm{E} 07$

namy $(1: 6)=\cdot 471101$.

nuclib=3

ELSE

$m=110.0-87.459 / 931.5016$

Thalf $=2.460 \mathrm{E} 01$

namy $(1: 6)=' 471100$.

nuclib $=3$

ENDIF

ELSEIF (name (1:5),EQ. 'AM240') THEN

$m=240.0+51.498 / 931.5016$

Thalf $=1.829 \mathrm{e} 05$

namy $(1: 6)=952400$.

nucli $b=2$

ELSEIF (name (1:5) .EQ. 'AM241') THEN $\mathrm{m}=241.0+52.931 / 931.5016$

Thalf $=1.364 \mathrm{e} 10$

namy $(1: 6)=\cdot 952410$.

nuclib $=2$

ELSEIF (name (1:5) .EQ. 'AM242') THEN

$m=242.0+55.463 / 931.5016$

Thalf $=5.767 \mathrm{e} 04$

namy $(1: 6)=952420^{\circ}$

nuclib $=2$

ELSEIF (name (1:5) .EQ. 'AM243') THEN

POST 1473

POST 1474

POST 1475

POST 1476

POST 1477

POST 1478

POST 1479

POST 1480

POST 1481

POST1482

POST 1483

POST 1484

POST1485

POST1486

POST 1487

POST 1488

POST 1489

POST 1490

POST 1491

POST 1492

POST 1493

POST 1494

POST 1495

POST 1496

POST 1497

POST 1498

POST1499

POST 1500

POST1501

POST 1502

POST 1503

POST 1504

POST 1505

POST 1506

POST 1507

POST 1508

POST 1509

POST 1510

POST1511

POST1512

POST1513

POST1514

POST1515

POST1516

POST1517

POST1518

POST1519

POST1520 
$\mathrm{m}=243.0+57.169 / 931.5016$

POST1521

Thalf $=2.329 \mathrm{el1}$

namy $(1: 6)=' 952430^{\prime}$

nuclib $=2$

ELSEIF (name (1:5) .EQ. 'AM244') THEN

$m=244.0+59.877 / 931.5016$

Thalf $=3.636 \mathrm{e} 04$

namy $(1: 6)=\cdot 952440$ '

nuclib $=2$

ELSEIF (name $(1: 5), E Q . \cdot A M 245$ ') THEN $m=245.0+61.891 / 931.5016$

Thalf $=7.452 \mathrm{e} 03$

$\operatorname{namy}(1: 6)=.952450^{\prime}$ nuclib $=2$

ELSEIF (name (1:5).EQ. 'AM246') THEN $m=246.0+64.990 / 931.5016$

Thalf $=1.500 \mathrm{e} 03$

namy $(1: 6)=\cdot 952460$ nuclib $=2$

ELSEIF (name (1:5).EO. 'AT217') THEN $\mathrm{m}=217.0+4.383 / 931.5016$

Thalf $=3.230 \mathrm{e}-02$

namy $(1: 6)=1852170$.

nuclib $=2$

ELSEIF (name (1:6) .EQ. 'BA137M') THEN

$m=137.0-87.070 / 931.5016$

Thalf $=1.531 \mathrm{e02}$

namy $(1: 6)=' 561371$

nuclib $=1$

ELSEIF (name (1:5) .EQ.'BI210') THEN $\mathrm{m}=210.0-14.815 / 931.5016$

Thalf $=4.330 \mathrm{e} 05$

namy $(1: 6)=\cdot 832100^{\circ}$

nucl ib $=2$

ELSEIF (name (1:5) .EQ. 'BI211') THEN $m=211.0-11.873 / 931.5016$

Thalf $=1.278 \mathrm{e} 02$

namy $(1: 6)=' 832110^{\prime}$

nucli $b=2$

EISEIF (name $(1: 5)$.EO. 'BI212') THEN $\mathrm{m}=212.0-8.142 / 931.5016$

Thalf $=3.633 \mathrm{e} 03$

$\operatorname{namy}(1: 6)=' 832130^{\circ}$

nucl ib $=2$

ELSEIF (name (1:5) .EQ. 'BI213') THEN

$\mathrm{m}=213.0-5.244 / 931.5016$

Thalf $=2.739 \mathrm{e} 03$

namy $(1: 6)=\cdot 832130$.

nuclib=2

ELSEIF (name (1:5) .EQ. 'BI214') THEN

$\mathrm{m}=214.0-1.218 / 931.5016$

Thalf $=1.194 \mathrm{e} 03$

namy $(1: 6)=\cdot 832140$ '

nuclib=2

ELSEIF (name (1:5).EQ. 'BK249') THEN

$\mathrm{m}=249.0+69.843 / 931.5016$

Thalf $=2.765 \mathrm{e} 07$

namy $(1: 6)=\cdot 972490$.

nuclib $=2$

ELSEIF (name (1:5).EO. 'BK250') THEN $m=250.0+72.951 / 931.5016$

Thalf $=1.160 \mathrm{e} 04$

namy $(1: 6)=' 972500$ '

nuclib $=2$

ELSEIF (name (1:3) .EQ.'C14') THEN

$\mathrm{m}=14.0+3.02 / 931.5016$

Thalf $=1.808 \mathrm{e} 11$

namy $(1: 6)=\cdot 060140^{\prime}$

nuclib=1

ELSEIF (name (1:5).EQ. 'CD109') THEN

$m=109.0-88.507 / 931.5016$

Thal $\mathrm{f}=4.009 \mathrm{e} 07$

namy $(1: 6)=1481090$.

nuclib=1

ELSEIF (name (1:6).EQ. 'CD113M') THEN $m=113.0-88.786 / 931.5016$

POST1522

POST1523

POST1524

POST1525

POST1526

POST1527

POST1528

POST1529

POST 1530

POST1531

POST1532

POST1533

POST1534

POST1535

POST1536

POST1537

POST1538

POST1539

POST1540

POST1541

POST1542

POST1543

POST1544

POST1545

POST1546

POST 1547

POST 1548

POST1549

POST1550

POST1551

POST1552

POST 1553

POST1554

POST 1555

POST 1556

POST1557

POST1558

POST1559

POST1560

POST1561

POST 1562

POST1563

POST1564

POST1565

POST1566

POST 1567

POST 1568

POST1569

POST 1570

POST1571

POST 1572

POST1573

POST1574

POST1575

POST1576

POST1577

POST1578

POST1579

POST1580

POST1581

POST1582

POST1583

POST1584

POST1585

POST1586

POST 1587

POST1588

POST 1589

POST 1590

POST1591

POST1592

POST1593

POST1594

POST1595

POST1596 
Thal $f=4.604 \mathrm{E} 08$

namy $(1: 6)=' 481131$ '

nuclib $=3$

ELSEIF (name (1:5).EQ. 'CE144') THEN $\mathrm{m}=144.0-80.441 / 931.5016$

Thalf $=2.456 \mathrm{e} 07$

namy $(1: 6)=' 581440^{\prime}$

nucli $b=1$

ELSEIF (name (1:5) .EQ. 'CF249') THEN

$m=249.0+69.717 / 931.5016$

Thalf $=1.106 \mathrm{e} 10$

namy $(1: 6)=\cdot 982490$.

nuclib $=2$

ELSEIF (name (1:5).EQ. 'CF250') THEN $\mathrm{m}=250.0+71.167 / 931.5016$

Thalf $=4.128 \mathrm{e} 08$

namy $(1: 6)=\cdot 982500$ '

nuclib $=2$

ELSEIF (name (1:5).EQ. 'CF251') THEN $\mathrm{m}=251.0+74.129 / 931.5016$

Thalf $=2.834 \mathrm{e} 10$

namy $(1: 6)=' 982510^{\prime}$

nuclib $=2$

ELSEIF (name (1:5).EQ. 'CF252') THEN $\mathrm{m}=252.0+76.03 / 931.5016$

Thalf $=8.325 \mathrm{e} 07$

namy $(1: 6)=' 982520^{\prime}$

nuclib $=2$

ELSEIF (name (1:5) .EQ. 'CM242') THEN

$\mathrm{m}=242.0+54.800 / 931.5016$

Thalf $=1.410 \mathrm{e} 07$

$\operatorname{namy}(1: 6)=' 962420^{\prime}$

nuclib $=2$

ELSEIF (name (1:5) .EQ. 'CM243') THEN

$\mathrm{m}=243.0+57.177 / 931.5016$

Thal $f=8.994 \mathrm{e} 08$

namy $(1: 6)=' 962430^{\circ}$

nuclib=2

ELSEIF (name (1:5) .EQ. 'CM244') THEN

$\mathrm{m}=244.0+58.449 / 931.5016$

Thalf $=5.715 e 08$

namy $(1: 6)=' 962440^{\prime}$

nuclib $=2$

ELSEIF (name (1:5) .EO. 'CM245') THEN $\mathrm{m}=245.0+60.998 / 931.5016$

Thalf $=2.682 \mathrm{el1}$

$\operatorname{namy}(1: 6)={ }^{\prime} 962450$ '

nuclib $=2$

ELSEIF (name (1:5) .EQ. 'CM246') THEN

$\mathrm{m}=246.0+62.614 / 931.5016$

Thal $f=1.493 \mathrm{el}$

$\operatorname{namy}(1: 6)=1962460^{\circ}$

nuclib $=2$

ELSEIF (name (1:5) .EQ. 'CM248') THEN

$m=248.0+67.388 / 931.5016$

Tha1f $=1: 070 \mathrm{el} 3$

namy $(1: 6)=962480$.

nucl $i b=2$

ELSEIF (name $(1: 5)$.EQ. 'CM250') THEN $m=250.0+72.985 / 931.5016$

Thalf $=5.491 \mathrm{e} 11$

$\operatorname{namy}(1: 6)=\cdot 962500$.

nuclib $=2$

ELSEIF (name (1:4).EO. 'CO58') THEN $\mathrm{m}=58.0-59.844 / 931.5016$

Thalf $=6.115 \mathrm{e} 06$

namy $(1: 6)=' 270580^{\prime}$

nuclib $=1$

ELSEIF (name (1:4).EQ. 'C060') THEN

$\mathrm{m}=60.0-61.646 / 931.5016$

Thalf $=1.663 \mathrm{e} 08$

namy $(1: 6)=\cdot 270600$ '

nuclib $=1$

ELSEIF (name (1:4) .EQ. 'CR51') THEN $\mathrm{m}=51.0-51.447 / 931.5016$

Thalf $=2.394 \mathrm{e} 06$

POST1597

POST1598

POST1599

POST1600

POST1601

POST1602

POST1603

POST1 604

POST1605

POST1606

POST 1607

POST1608

POST1609

POST 1610

POST'1611

POST1612

POST1613

POST1614

POST1615

POST1616

POST 1617

POST1618

POST1619

POST 1620

POST1621

POST 1622

POST 1623

POST1624

POST 1625

POST 1626

POST 1627

POST1628

POST 1629

POST 1630

POST1631

POST1632

POST 1633

POST 1634

POST1635

POST 1636

POST1637

POST1638

POST1639

POST1640

POST1641

POST 1642

POST 1643

POST 1644

POST 1645

POST 1646

POST1647

POST 1648

POST1649

POST 1650

POST1651

POST 1652

POST 1653

POST 1654

POST1 1655

POST1656

POST 1657

POST1658

POST1659

POST 1660

POST1661

POST 1662

POST1 1663

POST1 664

POST1665

POST 1666

POST 1667

POST 1668

POST 1669

POST 1670

POST1671

POST1672 
$\operatorname{namy}(1: 6)=' 240510^{\prime}$

nuclib $=1$

ELSEIF (name (1:5).EQ.'CS134') THEN $m=134.0-86.906 / 931.5016$

Thalf $=6.507 \mathrm{e} 07$

namy $(1: 6)={ }^{\prime} 551340^{\circ}$

nuclib $=1$

ELSEIF (name ( $1: 5) . E Q . ' C S 135$ ') THEN

$m=135.0-87.662 / 931.5016$

Thalf $=7.258 \mathrm{E} 13$

$\operatorname{namy}(1: 6)=' 551350$

nucl $i b=1$

ELSEIF (name (1:5) .EQ.:CS137') THEN

$m=137.0-86.556 / 931.5016$

Thalf $=9.467 \mathrm{e} 08$

namy $(1: 6)=\cdot 551370^{\prime}$

nucl $i b=1$

ELSEIF (name (1:5). EQ. 'ES253') THEN $m=253.0+79.007 / 931.5016$

Thalf $=1.769 \mathrm{e} 06$

$\operatorname{namy}(1: 6)={ }^{\prime} 992530^{\circ}$

nuclib $=2$

ELSEIF (name ( $1: 5)$. EO. 'ES254') THEN IF (name $(6: 6)$. EQ. 'M') THEN $m=254.0+82.072 / 931.5016$

Thalf $=1.415 \mathrm{e} 05$

namy $(1: 6)=1992541^{\prime}$

ELSE nuclib $=2$

$\mathrm{m}=254.0+81.994 / 931.5016$

Thal $f=2.382 \mathrm{e} 07$

namy $(1: 6)=\cdot 992540$

ENDIF

nuclib $=2$

ELSEIF (name (1:5).EQ. 'EU150') THEN $m=150.0-74.800 / 931.5016$

Thalf $=1.136 \mathrm{e} 09$

$\operatorname{namy}(1: 6)=631500^{\circ}$

nuclib $=3$

ELSEIF (name (1:5) .EO. 'EU152') THEN

$\mathrm{m}=152.0-72.899 / 931.5016$

Thalf $=4.292 \mathrm{e} 08$

namy $(1: 6)=\cdot 631520$.

nuclib $=3$

ELSEIF (name (1:5) EO. 'EU154') THEN $m=154.0-71.748 / 931.5016$

Thalf $=2.714 \mathrm{e} 08$

$\operatorname{namy}(1: 6)=' 631540^{\prime}$

nuclib $=3$

ELSEIF (name (1:5) .EQ. 'EU155') THEN

$m=155.0-71.829 / 931.5016$

Thalf $=1.565 \mathrm{e} 08$

namy $(1: 6)=' 631550^{\circ}$

nuclib $=3$

ELSEIF (name (1:4).EQ.'FE55') THEN

$\mathrm{m}=55.0-57.476 / 931.5016$

Thalf $=8.2048 \mathrm{e} 07$

$\operatorname{namy}(1: 6)=\cdot 260550$.

nuclib $=1$

ELSEIF (name (1:4).EQ. 'FE59') THEN

$\mathrm{m}=59.0-60.661 / 931.5016$

Thalf $=3.888 \mathrm{e} 06$

namy $(1: 6)=' 260590^{\prime}$

nuclib=1

ELSEIF (name (1:5) .EO. 'GD152') THEN

$m=152.0-74.718 / 931.5016$

Thalf $=3.408 \mathrm{E} 21$

$\operatorname{namy}(1: 6)=641520^{\circ}$

nuclib $=1$

ELSEIF (name (1:5) .EQ. ' GD162 ') THEN

$m=162.0-64.240 / 931.5016$

Thalf $=6.000 \mathrm{E} 02$

$\operatorname{namy}(1: 6)=641620$.

nuclib=1

ELSEIF (name (1:2) .EQ. 'H3') THEN $m=3.0+14.95 / 931.5016$

POST1676

POST1677

POST1678

POST 1679

POST 1680

POST1681

POST 1682

POST 1683

POST1684

POST 1685

POST 1686

POST 1687

POST 1688

POST 1689

POST1 1690

POST1691

POST1 1692

POST1693

POST1694

POST 1695

POST1696

POST 1697

POST1698

POST1699

POST 1700

POST1701

POST1702

POST1703

POST1704

POST1705

POST1706

POST 1707

POST1708

POST1709

POST 1710

POST1711

POST1712

POST1713

POST 1714

POST1715

POST 1716

POST 1717

POST1718

POST 1719

POST 1720

POST1721

POST 1722

POST 1723

POST1724

POST1725

POST 1726

POST1727

POST1728

POST1729

POST1730

POST1731

POST 1732

POST1733

POST1734

POST1735

POST 1736

POST1737

POST1738

POST1739

POST 1740

POST1741

POST 1742

POST 1743

POST1744

POST 1745

POST 1746

POST 1747

POST 1748 
Thalf $=3.897 \mathrm{e} 08$

namy $(1: 6)=' 010030^{\prime}$

nuclib=1

ELSEIF (name $(1: 4)$. EQ. 'I129') THEN

$\mathrm{m}=129.0-88.507 / 931.5016$

Thalf $=4.954 \mathrm{e} 14$

$\operatorname{namy}(1: 6)=\cdot 531290^{\circ}$

nuclib=1

ELSEIF (name (1:4).EQ.'KR85') THEN

$\mathrm{m}=85.0-81.477 / 931.5016$

Thalf $=3.383 \mathrm{e} 08$

$\operatorname{namy}(1: 6)=1360850$.

nuclib $=1$

ELSEIF (name(1:4). EQ.'MN54') THEN $\mathrm{m}=54.0-55.553 / 931.5016$

Thalf $=2.700 \mathrm{e} 07$

namy $(1: 6)=' 250540$. nuclib $=1$

ELSEIF (name (1:4).EQ.'NB95') THEN

IF (name (5:5). EQ.' 'M') THEN $\mathrm{m}=95.0-86.547 / 931.5016$

Thalf $=3.118 \mathrm{E} 05$

namy $(1: 6)=\cdot 410951$ '

nuclib $=1$

ELSE

$\mathrm{m}=95.0-86.783 / 931.5016$

Thalf $=3.037 \mathrm{e} 06$

namy $(1: 6)={ }^{\prime} 410950$ '

nucli $b=1$

ENDIF

ELSEIF (name (1:4) .EQ.'NI59') THEN

$\mathrm{m}=59.0-61.153 / 931.5016$

Thalf $=2.5246 \mathrm{el}$

namy $(1: 6)=' 280590$ ' nucli $b=1$

ELSEIF (name $(1: 4)$. EQ. 'NI63') THEN $\mathrm{m}=63.0-65.512 / 931.5016$

Thalf $=2.9032 \mathrm{e} 9$

namy $=$ ' 280630 nucli $b=1$

ELSEIF (name (1:5) .EQ. 'NP237') THEN $m=237.0+44.868 / 931.5016$

Thalf $=6.753 \mathrm{e} 13$

namy $=$ ' $932370^{\circ}$

nuclib $=2$

EISEIF (name (1:5).EQ. 'NP239') THEN

$\mathrm{m}=239.0+49.306 / 931.5016$

Thalf $=2.035 \mathrm{e} 05$

namy $=.932390$

nuclib $=2$

ELSEIF (name (1:5).EQ.'NP240') THEN

IF (name $(6: 6) . E Q$.' $M$ ') THEN

$\mathrm{m}=240.0+52.321 / 931.5016$

Thalf $=4.440 \mathrm{e} 02$

namy $=$ ' 932401

ELSE nuclib $=2$

$\mathrm{m}=240.0+52.321 / 931.5016$

Thalf $=3.900 \mathrm{e} 03$

namy $=$ ' 932400 '

ENDIF

nuclib $=2$

ELSEIF (name (1:5) .EQ. 'PA231') THEN

$\mathrm{m}=231.0+33.422 / 931.5016$

Thalf $=1.034 \mathrm{e} 12$

namy $=$ ' $912310^{\prime}$

nuclib=2

ELSEIF (name (1:5) .EQ. 'PA233') THEN $\mathrm{m}=233.0+37.485 / 931.5016$

Thalf $=2.333 \mathrm{e} 06$

namy $=$ ' $912330^{\circ}$

nuclib $=2$

ELSEIF (name (1:5).EQ.'PA234') THEN

IF (name $(6: 6) . E Q . ' M$ ') THEN $\mathrm{m}=234.0+40.408 / 931.5016$

Thalf $=7.020 \mathrm{e} 01$

POST1754

POST 1755

POST 1756

POST1757

POST 1758

POST1759

POST 1760

POST1761

POST 1762

POST 1763

POST1764

POST1765

POST1766

POST 1767

POST1768

POST1769

POST 1770

POST 1771

POST1772

POST1773

POST1774

POST1775

POST1776

POST1777

POST 1778

POST1779

POST1780

POST1781

POST1782

POST1783

POST1784

POST 1785

POST1786

POST1787

POST1788

POST1789

POST1790

POST1791

POST 1792

POST1793

POST1794

POST1795

POST1796

POST 1797

POST1798

POST1799

POST 1800

POST1801

POST1802

POST 1803

POST1804

POST 1805

POST 1806

POST 1807

POST1808

POST 1809

POST 1810

POST1811

POST 1812

POST 1813

POST1814

POST1815

POST 1816

POST1817

POST 1818

POST1819

POST 1820

POST1821

POST 1822

POST1823

POST1824 
namy = ' 912341

nuclib $=2$

ELSE

$m=234.0+40.334 / 931.5016$

Thalf $=2.412 \mathrm{e} 04$

namy $=$ ' 912340 '

nuclib $=2$

ENDIF

ELSEIF (name (1:5) .EQ.'PB209') THEN

$m=209.0-17.638 / 931.5016$

Thalf $=1.188 \mathrm{e} 04$

namy = ' $822090^{\prime}$

nuclib $=2$

ELSEIF (name (1:5) .EQ.'PB210') THEN

$m=210.0-14.752 / 931.5016$

Thalf $=7.037 \mathrm{e} 08$

namy $=\cdot 822100$

nuclib $=2$

ELSEIF (name $(1: 5)$.EQ. 'PB211') THEN

$\mathrm{m}=211.0-10.494 / 931.5016$

Thalf $=2.166 \mathrm{e} 03$

namy $=$ ' $822110^{\prime}$

nuclib $=2$

ELSEIF (name (1:5).EQ.'PB212') THEN $\mathrm{m}=212.0-7.571 / 931.5016$

Thalf $=3.830 \mathrm{e} 04$

namy $=\cdot 822120^{\prime}$

nuclib $=2$

ELSEIF (name (1:5).EQ.'PB214') THEN $\mathrm{m}=214.0-.188 / 931.5016$

Thalf $=1.608 \mathrm{e} 03$

namy $=' 822140$ '

nuclib $=2$

ELSEIF (name (1:5) .EQ.'PD107') THEN

$m=107.0-88.374 / 931.5016$

Thal $f=2.050 \mathrm{E} 14$

namy $=\cdot 461070$

nuclib=1

ELSEIF (name (1:5).EQ.'PM147') THEN

$m=147.0-79.052 / 931.5016$

Thalf $=8.279 \mathrm{e} 07$

namy $=\cdot 611470$ '

nuclib=1

ELSEIF (name (1:5).EQ.'PO210') THEN $\mathrm{In}=210.0-15.977 / 931.5016$

Thalf $=1.196 \mathrm{e} 07$

namy = ' $842100^{\prime}$

nuclib $=2$

ELSEIF (name (1:5). EQ.'PO211') THEN $\mathrm{m}=211.0-12.457 / 931.5016$

Thalf $=5.600 \mathrm{e}-01$

namy $=$ ' 842110 '

nuc 1 ib $=2$

ELSEIF (name (1:5) .EQ.'PO212') THEN $m=212.0-10.394 / 931.5016$

Thalf $=3.000 e-07$

nany $=$ ' 842120 '

nuclib=2

ELSEIF (name (1:5) .EQ.'PO213') THEN $\mathrm{m}=213.0-6.676 / 931.5016$

Thalf $=4.200 e-06$

namy $=$ ' 842130 ' nuclib $=2$

ELSEIF (name (1:5) .EQ. 'PO214') THEN $m=214.0-4.493 / 931.5016$

Thalf $=1.643 \mathrm{e}-04$

namy $={ }^{\circ} 842140^{\circ}$

nuclib=2

ELSEIF (name (1:5) .EQ.'PO215') THEN $\mathrm{m}=215.0-0.542 / 931.5016$

Thalf $=1.780 \mathrm{e}-03$

namy $=$ ' $842150^{\circ}$

nuclib $=2$

ELSEIF (name (1:5) .EQ. 'PO216') THEN $m=216.0+1.760 / 931.5016$

Thalf $=1.500 \mathrm{e}-01$

POST1825

POST 1826

POST 1827

POST1828

POST1829

POST1830

POST1831

POST1 1832

POST1833

POST 1834

POST 1835

POST1836

POST1 1837

POST1838

POST1839

POST1840

POST1841

POST1 1842

POST 1843

POST1 1844

POST 1845

POST1846

POST1847

POST1848

POST1849

POST 1850

POST1851

POST1 852

POST1853

POST1854

POST1 855

POST1856

POST1857

POST1858

POST1859

POST1860

POST1861

POST 1862

POST 1863

POST1864

POST1865

POST1 866

POST1867

POST1 1868

POST1 869

POST1870

POST1871

POST1872

POST1873

POST1874

POST1875

POST1876

POST1877

POST1878

POST1879

POST 1880

POST1881

POST1882

POST1883

POST1884

POST1885

POST1886

POST1 1887

POST1888

POST1 889

POST1890

POST1891

POST1892

POST1893

POST1894

POST1895

POST1896

POST1 897

POST1898

POST1899

POST 1900 
namy $=\cdot 842160^{\circ}$

nuclib $=2$

ELSEIF (name (1:5). EQ.'PO218') THEN $\mathrm{m}=218.0+8.351 / 931.5016$

Thal $f=1.830 \mathrm{e} 2$

namy $=\cdot 842180$

nucli $i b=2$

ELSEIF (name (1:5) .EQ.'PR144') THEN $m=144.0-80.76 / 931.5016$

Thal $f=1.037 \mathrm{e} 03$

namy $=\cdot 591440$ '

nuclib $=1$

ELSEIF (name (1:5) .EQ. 'PU236') THEN $m=236.0+42.879 / 931.5016$

Thalf $=8.997 \mathrm{e} 07$

namy $=.942360^{\circ}$ nuclib $=2$

ELSEIF (name (1:5) .EQ.'PU238') THEN $\mathrm{m}=238.0+46.16 / 931.5016$

Thalf $=2.769 \mathrm{e} 09$

namy $=\cdot 942380$ nuclib $=2$

ELSEIF (name (1:5) .EQ.'PU239') THEN $\mathrm{m}=239.0+48.584 / 931.5016$ Tha If $=7.594 \mathrm{el1}$ namy $=\cdot 942390$ nuclib=2

ELSEIF (name (1:5) .EQ. 'PU240') THEN $m=240.0+50.122 / 931.5016$

Thalf $=2.063 \mathrm{ell}$ namy $=$ ' 942400 nuclib=2

ELSEIF (name (1:5).EQ.'PU241') THEN $m=241.0+52.952 / 931.5016$

Thalf $=4.544 \mathrm{e} 08$ namy $=$ ' 942410 nuclib $=2$

ELSEIF (name (1:5) .EQ. 'PU242') THEN $\mathrm{m}=242.0+54.713 / 931.5016$

Thalf $=1.221 \mathrm{e} 13$

namy $=$ ' $942420^{\prime}$ nuclib=2

ELSEIF (name (1:5).EQ. 'PU244') THEN $\mathrm{m}=244.0+59.802 / 931.5016$

Thalf $=2.607 \mathrm{e} 15$

namy $=$ ' 942440

nuclib $=2$

ELSEIF (name (1:5) .EQ. 'PU246') THEN $m=246.0+65.391 / 931.5016$

Thalf $=9.374 \mathrm{e} 05$

namy $=$ ' 942460

nuclib $=2$

ELSEIF (name (1:5) .EQ. 'RA223') THEN $\mathrm{m}=223.0+17.232 / 931.5016$

Thalf $=9.879 \mathrm{e} 05$

namy $=' 882230^{\prime}$ nuclib=2

ELSEIF (name (1:5).EQ.'RA224') THEN $m=224.0+18.804 / 931.5016$

Thal $f=3.162 \mathrm{e} 05$

namy $=\cdot 882240^{\prime}$ nuclib $=2$

ELSEIF (name (1:5) ,EO. 'RA225') THEN $m=225.0+21.988 / 931.5016$ Thalf $=1.279 \mathrm{e} 06$ namy $=$ ' $882250^{\prime}$ nuclib=2

ELSEIF (name (1:5) .EQ.'RA226') THEN $m=226.0+23.662 / 931.5016$

Thalf $=5.049 \mathrm{e} 10$

namy $=\cdot 882260$ ' nuclib $=2$

ELSEIF (name (1:5).EQ. 'RA228') THEN $\mathrm{m}=228.0+28.936 / 931.5016$ Thalf $=2.1143 \mathrm{e} 08$ namy $=$ ' 882280 '

POST 1901

POST1902

POST1903

POST1904

POST1905

POST1906

POST1907

POST1908

POST1909

POST1910

POST1911

POST1912

POST1913

POST1914

POST1915

POST1916

POST1917

POST1918

POST1919

POST1920

POST1921

POST1922

POST1923

POST1924

POST1925

POST1926

POST1927

POST1928

POST1929

POST1930

POST1931

POST1932

Post1933

PosT1934

POST1935

POST1936

POST1937

POST1938

POST1939

POST1940

POST1941

POST1942

POST1943

POST1944

POST1945

POST1946

POST1947

POST1948

POST1949

POST1950

POST1951

POST1952

POST1953

POST1954

POST 1955

POST 1956

POST1957

POST1958

POST1959

POST1960

POST1: $: 1$

POST1962

POST1963

POST1964

POST1965

POST 1966

POST1967

POST 1968

POST1969

POST1970

POST 1971

POST1972

POST1973

POST1974

POST1975

POST1976 
nuclib $=2$

ELSEIF (name (1:5).EQ. 'RH106') THEN

$m=106.0-86.365 / 931.5016$

Thalf $=29.9$

namy $={ }^{\prime} 451060^{\circ}$

nuclib $=1$

ELSEIF (name (1:5).EQ.'RN219') THEN

$m=219.0+8.828 / 931.5016$

Thalf $=3.960 \mathrm{e} 00$

namy $=\cdot 862190$

nuclib $=2$

ELSEIF (name (1:5).EQ. 'RN220') THEN $\mathrm{m}=220.0+10.590 / 931.5016$

Thalf $=5.560 \mathrm{e} 01$

namy $={ }^{\prime} 862200^{\prime}$

nuclib $=2$

ELSEIF (name (1:5).EQ. 'RN222') THEN $\mathrm{m}=222.0+16.367 / 931.5016$

Thalf $=3.304 \mathrm{e} 05$

namy $=\cdot 862220$

nucli $b=2$

ELSEIF (name (1:5).EQ. 'RU106') THEN $\mathrm{m}=106.0-86.326 / 931.5016$

Thalf $=3.181 \mathrm{e} 07$

namy $=$ ' $441060^{\prime}$

muclib $=1$

ELSEIF (name (1:5) .EQ.'SB125') THEN

$m=125.0-88.258 / 931.5016$

Thal $\mathrm{f}=8.741 \mathrm{e} 07$

namy $=$ ' 511250

nuclib=1

ELSEIF (name (1:5).EQ. 'SB126') THEN

IF (NAME $(6: 6)$. EQ. ' $M$ ') THEN

$\mathrm{m}=126.0-86.382 / 931.5016$

Thal $f=1.140 \mathrm{E} 03$

namy $=$ ' 511261 '

nuclib $=1$

ELSE

$m=126.0-86.400 / 931.5016$

Thal $f=1.071 \mathrm{E} 06$

namy $=\cdot 511260$

nuclib $=1$

ENDIF

ELSEIF (name (1:4) .EQ.'SE79') THEN

$m=79.0-75.920 / 931.5016$

Thalf $=2.050 \mathrm{E} 12$

narny $=1340790$

nuclib=1

ELSEIF (name (1:5) .EQ.'SM147') THEN

$\mathrm{m}=147.0-79.276 / 931.5016$

Thalf $=3.377 \mathrm{el} 8$

namy $=' 621470$

nuclib=1

ELSEIF (name (1:5) .EQ.' SM148') THEN

$m=148.0-79.346 / 931.5016$

Thalf $=2.525 \mathrm{e} 23$

narny $=$ ' $621480^{\prime}$

nuclib $=1$

ELSEIF (name (1:5) .EO. 'SM151') THEN $\mathrm{m}=151.0-74.587 / 931.5016$

Thalf $=2.840 \mathrm{e} 09$

namy $=$ ' 621510 '

nuclib $=1$

ELSEIF (name $(1: 6)$. EQ.' SN119M') THEN

$m=119.0-89.978 / 931.5016$

Thalf $=2.117 \mathrm{E} 07$

namy $=$ ' 501191

nuclib=1

ELSEIF (name $(1: 6) . E Q$. 'SN121M') THEN $m=121.0-89.197 / 931.5016$

Thalf $=1.577 \mathrm{E} 09$

namy $=$ ' 501211'

nuclib $=1$

ELSEIF (name $(1: 6)$.EQ. 'SN123M') THEN $m=123.0-87.795 / 931.5016$

Thalf $=2.405 \mathrm{E} 03$

POST 1979

POST 1980

POST1981

POST 1982

POST1983

POST1984

POST 1985

POST1986

POST1987

POST1988

POST1989

POST1990

POST1991

POST1992

POST1993

POST1994

POST1995

POST1996

POST1997

POST1998

POST 1999

POST2000

POST2001

POST2002

POST2003

POST2004

POST2005

POST2006

POST2007

POST2008

POST2009

POST2010

POST2011

POST2012

POST2013

POST2014

POST2015

POST2016

POST2017

POST 2018

POST2019

POST2020

POST2021

POST2022

POST2023

POST2024

POST2025

POST2026

POST2027

POST 2028

POST2 029

POST2 030

POST2031

POST2032

POST2033

POST2034

POST2035

POST2036

POST2037

POST2038

POST2039

POST2 040

POST2041

POST2042

POST2043

POST2044

POST2045.

POST2046

POST2 047

POST2048

POST2049

POST2050

POST2051

POST 2052 
namy $=$ ' 501231

nuclib=1

ELSEIF (name (1:5) .EQ. 'SN126') THEN $m=126.0-86.021 / 931.5016$

Thalf $=3.156 \mathrm{E} 12$

namy $=$ ' $501260^{\circ}$

nuclib $=3$

ELSEIF (name (1:4).EQ. 'SR89') THEN

$m=89.0-86.211 / 931.5016$

Thal $f=4.363 \mathrm{e} 06$

namy $=$ ' $380890^{\circ}$ nuclib=1

ELSEIF (name (1:4) .EQ.' SR90') THEN $\mathrm{m}=90.0-85.942 / 931.5016$

Thalf $=9.190 \mathrm{e} 08$

namy $=$ ' 380900 '

nuclib=1

ELSEIF (name (1:5).EQ.'TA182') THEN

$m=182.0-46.436 / 931.5016$

Thalf $=9.936 \mathrm{e} 06$

namy $=\cdot 731820$

nuclib $=1$

ELSEIF (name (1:5).EQ.' TB162') THEN $m=162.0-65.680 / 931.5016$

Thalf $=4.482 \mathrm{E} 02$

namy $=$ ' 651620 '

nuclib $=1$

ELSEIF (name (1:4).EQ. 'TC99') THEN

$m=99.0-87.324 / 931.5016$

Thal $f=6.722 \mathrm{el} 2$

namy $=$ ' 430990 '

nuclib=1

ELSEIF (name (1:6).EQ. 'TE125M') THEN $m=125.0-88.879 / 931.5016$

Thalf $=5.011 \mathrm{e} 06$

namy $=$ ' 521251 '

nuclib=1

ELSEIF (name (1:5).EQ. 'TE127') THEN

IF (NAME $(6: 6)$. EQ. 'M') THEN

$m=127.0-88.198 / 931.5016$

Thalf $=9.418 \mathrm{E} 06$

namy $=\cdot 521271$ '

nuclib=1

ELSE

$m=127.0-88.286 / 931.5016$

Thalf $=3.366 \mathrm{E} 04$

namy $=.521270^{\circ}$

nuclib $=1$

ENDIF

ELSEIF (name (1:5) .EO. 'TH227') THEN $\mathrm{m}=227.0+25.803 / 931.5016$

Thal $f=1.617 \mathrm{e} 06$

namy $=\cdot 902270^{\prime}$

nuclib $=2$

ELSEIF (name (1:5).EQ.'TH228') THEN

$m=228.0+26.749 / 931.5016$

Thalf $=6.037 \mathrm{e} 07$

namy $=$ ' $902280^{\prime}$

nuclib=2

ELSEIF (name (1:5) .EQ. 'TH229') THEN $m=229.0+29.581 / 931.5016$

Thalf $=2.316 \mathrm{e} 11$

namy $=$ ' 902290 '

nuclib=2

ELSEIF (name (1:5).EQ. 'TH230') THEN $\mathrm{m}=230.0+30.858 / 931.5016$

Thalf $=2.430 \mathrm{e} 12$

namy $=.902300$ '

nuclib $=2$

ELSEIF (name $(1: 5)$. EO. 'TH231') THEN $\mathrm{m}=231.0+33.812 / 931.5016$

Thalf $=9.187 \mathrm{e} 04$

namy $=\cdot 902310$ '

nuclib $=2$

ELSEIF (name (1:5).EQ. 'TH232') THEN $m=232.0+35.444 / 931.5016$

POST2053

POST2054

POST2055

POST2056

POST 2057

POST2 058

POST2059

POST2060

POST2061

POST2062

POST2063

POST2064

POST2065

POST2066

POST2067

POST2068

POST2069

POST 2070

POST2071

POST2072

POST2073

POST2074

POST2075

POST 2076

POST2077

POST 2078

POST2079

POST2080

POST2081

POST 2082

POST2083

POST2084

POST2085

POST2086

POST2087

POST2088

POST2089

POST2090

POST2091

POST2092

POST2093

POST2094

POST2095

POST2096

POST2097

POST2098

POST2099

POST2100

POST2101

POST2102

POST2103

POST2104

POST2105

POST2 106

POST2107

POST2 108

POST2 109

POST2110

POST2111

POST2 112

POST2 113

POST2114

POST2115

POST2116

POST2117

POST2118

POST2119

POST2120

POST2121

POST 2122

POST2123

POST2124

POST 2125

POST2126

POST2127

POST2128 
Thalf $=4.434 \mathrm{e} 17$

POST 2129 namy $=\cdot 902320^{\circ}$

nuclib=2

ELSEIF (name (1:5) .EQ.'TH234') THEN $\mathrm{m}=234.0+40.607 / 931.5016$

Thalf $=2.082 \mathrm{e} 06$

namy $=$ ' 902340 '

nuclib $=2$

ELSEIF (name (1:5). EQ.'TL207') THEN $m=207-21.049 / 931.5016$

Thalf $=2.862 \mathrm{e} 02$

$\operatorname{namy}(1: 6)=\cdot 812070^{\prime}$ nuclib $=2$

ELSEIF (name (1:5).EO.' TL208') THEN $m=208.0-16.774 / 931.5016$

Thal $\mathrm{f}=1.842 \mathrm{e} 02$

namy $(1: 6)={ }^{\prime} 812080^{\prime}$

nucli $b=2$

ELSEIF (name (1:5) .EQ.'TL209') THEN

$\mathrm{m}=209-13.652 / 931.5016$

Thalf $=1.320 \mathrm{e} 02$

namy $(1: 6)=\cdot 812090$

nuclib=2

ELSEIF (name (1:4) .EQ.' U232') THEN $\mathrm{m}=232.0+34.587 / 931.5016$

Thal $=2.272 \mathrm{e} 09$

namy $=\cdot 922320$ nuclib $=2$

ELSEIF (name ( $1: 4)$.EO. 'U233') THEN $\mathrm{m}=233.0+36.915 / 931.5016$

Thal $f=5.002 \mathrm{e} 12$

namy $=.922330$

nuclib $=2$

ELSEIF (name (1:4),EO. 'U234') THEN

$\mathrm{m}=234.0+38.141 / 931.5016$

Thalf $=7.716 \mathrm{e} 12$

namy $=\cdot 922340$

nuclib $=2$

ELSEIF (name ( $1: 4)$.EQ. 'U235') THEN

$m=235.0+40.915 / 931.5016$

Thalf $=2.221 \mathrm{e} 16$

namy $=$ ' 922350

nuclib $=2$

ELSEIF (name $(1: 4), E Q .^{\prime}$ U236') THEN

$\mathrm{m}=236.0+42.441 / 931.5016$

Thalf $=7.389 \mathrm{el}$

namy $=922360$

nuclib=2

ELSEIF (name (1:4) EO. 'U237') THEN $\mathrm{m}=237.0+45.387 / 931.5016$

Thalf $=5.832 \mathrm{e} 05$

namy $=\cdot 922370$

nuclib $=2$

ELSEIF (name (1:4).EQ.' U238') THEN

$\mathrm{m}=238.0+47.305 / 931.5016$

Thalf $=1.410 \mathrm{e} 17$

namy $=\cdot 922380$

nuclib $=2$

ELSEIF (name (1:4) EQ.'U240') THEN $\mathrm{m}=240.0+52.711 / 931.5016$

Thalf $=5.076 \mathrm{e} 04$

namy $=.922400$.

nuclib $=2$

ELSEIF (name (1:3) .EO. 'Y9O') THEN $m=90.0-86.488 / 931.5016$

Thalf $=2.304 \mathrm{e} 05$

namy $=$ : 390900

nuclib $=1$

ELSEIF (name $(1: 4)$.EQ.'ZN65') THEN

$\mathrm{m}=65.0-65.91 / 931.5016$

Thalf $=2.107 \mathrm{e} 07$

namy $=\cdot 300650$

nuclib=1

ELSEIF (name (1:4) .EQ.'ZR93') THEN $\mathrm{m}=93.0-87.120 / 931.5016$

Thalf $=4.828 \mathrm{E} 13$

POST 2130

POST2131

POST2132

POST2133

POST2134

POST 2135

POST2136

POST2137

POST2138

POST2139

POST2140

POST2141

POST2142

POST2143

POST2144

POST2145

POST 2146

POST 2147

POST 2148

POST2149

POST2150

POST2151

POST2152

POST2153

POST2154

POST2155

POST2156

POST2157

POST2158

POST2159

POST2160

POST2161

POST2162

POST2 163

POST2164

POST2165

POST2166

POST2167

POST2168

POST2169

POST2170

POST2171

POST2172

POST2173

POST 2174

POST2175

POST2176

POST 2177

POST2178

POST2179

POST2180

POST2181

POST2182

POST2183

POST2184

POST2185

POST2186

POST2187

POST2188

POST2189

POST2190

POST2191

POST2192

POST2193

POST2194

POST2195

POST2196

POST2197

POST2198

POST2199

POST2200

POST2201

POST2202

POST2203

POST2204 
C

namy $=\cdot 400930$

nucli $b=1$

ELSEIF (name (1:4).EQ. 'ZR95') THEN

$\mathrm{m}=95.0-85.659 / 931.5016$

Thalf $=5.528 \mathrm{e} 06$

namy $=.400950$.

ELSE

nuclib $=1$

$m=.5$

Thalf $=.05$

namy $=: \star \star \star \star \star \star * *$.

ENDIF

nuclib $=0$

C FROM THE BOOK "ATOMS; RADIATION, AND RADIATION PROTECTION"

C BY JAMES TURNER, USED A FORMULA TO CALCULATE SPECIFIC

C ACTIVITY IN BQ/G WHEN THE HALFLIFE WAS EXPRESSED IN SECONDS.

C

$$
\text { sa1 }=\left(6.022136736 \mathrm{e} 23^{*} \operatorname{alog}(2.0)\right) /\left(m^{*} \text { Thalf }\right)
$$

C THEN CONVERTED Bq/g TO $\mathrm{Ci} / \mathrm{g}$

$$
\mathrm{sa}=\operatorname{sal} / 3.7 \mathrm{e} 10
$$

C IT IS THEN POSSIBLE TO CONVERT A VALUE IN CURIES TO GRAMS

C BY THE FOLLOWING EQUATION.

C

$$
\text { activy }=\operatorname{activ} / s a
$$

RETURN

END

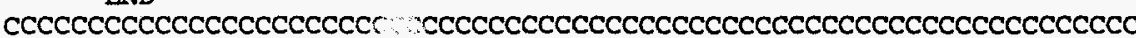

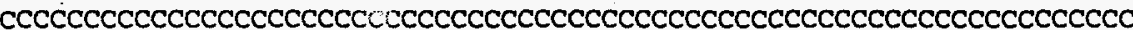
SUBROUTINE HEADER

WRITE $(6,9801)$

9801 FORMAT (

$+1234567890123456789012345678901234567890^{\prime}$
$+\quad \cdot 1234567890123456789012$

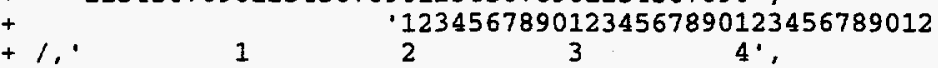

$+1$.

2

$5^{3}$

5

$6^{4}$

$+1$.

$+$

$+1$.

$+1, \cdot$

$>$

1.

$+1, \quad>$

$+1,1,>$

$1,1>>$

)

WRITE $(6,9802)$

RMAT

$$
\begin{array}{ll}
1,1 & > \\
1, \cdot \quad> \\
1, \cdot \quad>> \\
1, \cdot \\
+1, \cdot
\end{array}
$$$$
>>
$$$$
1,1>
$$

$$
\text { , }
$$$$
\text { , }
$$$$
\text { , }>>>>>>>>>>>>-----<<,
$$

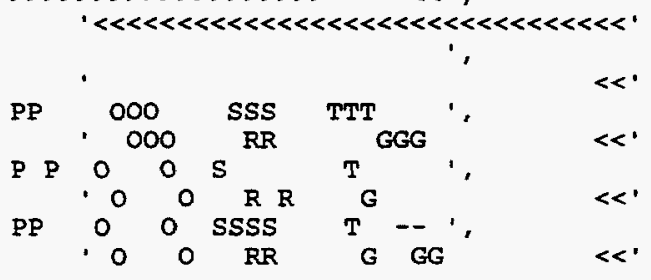$$
\begin{array}{lll}
P & 0 & 0 \\
P & 0 & 0 \\
P & 0 & 0 \\
P & 000 & 0
\end{array}
$$

R $\mathrm{R}$

$S$
$R$
$R$
$R$

$>>>$ 000 SSS

$$
\text { ' }>>>>>>>>>>>>>--\cdots--<<\text {, }
$$

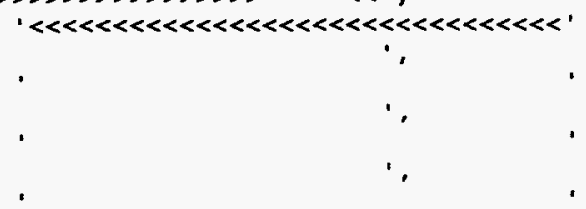

POST2208

POST2209

POST 2210

POST2211

POST 2212

POST 2213

POST2214

POST2215

POST2216

POST 2217

POST2218

POST2219

POST2220

POST2221

POST2222

POST2223

POST2224

POST2225

POST2226

POST2227

POST2228

POST2229

POST2230

PosT2231

POST2232

POST2233

POST2234

POST2235

POST2236

POST2237

POST 2238

POST2239

POST2240

POST2241

POST2242

POST 2243

POST2244

POST2245

POST2246

POST2247

POST 2248

POST2249

POST2250

POST2251

POST2252

POST2253

POST2254

POST2255

POST2256

POST 2257

POST2258

POST2259

POST 2260

POST2261

POST 2262

POST2263

POST2264

POST2265

POST2266

POST2267

POST 2268

POST 2269

POST 2270

POST 2271

POST 2272

POST 2273

POST2274

POST 2275

POST2276

POST2277

POST2278

POST2279

POST2280 

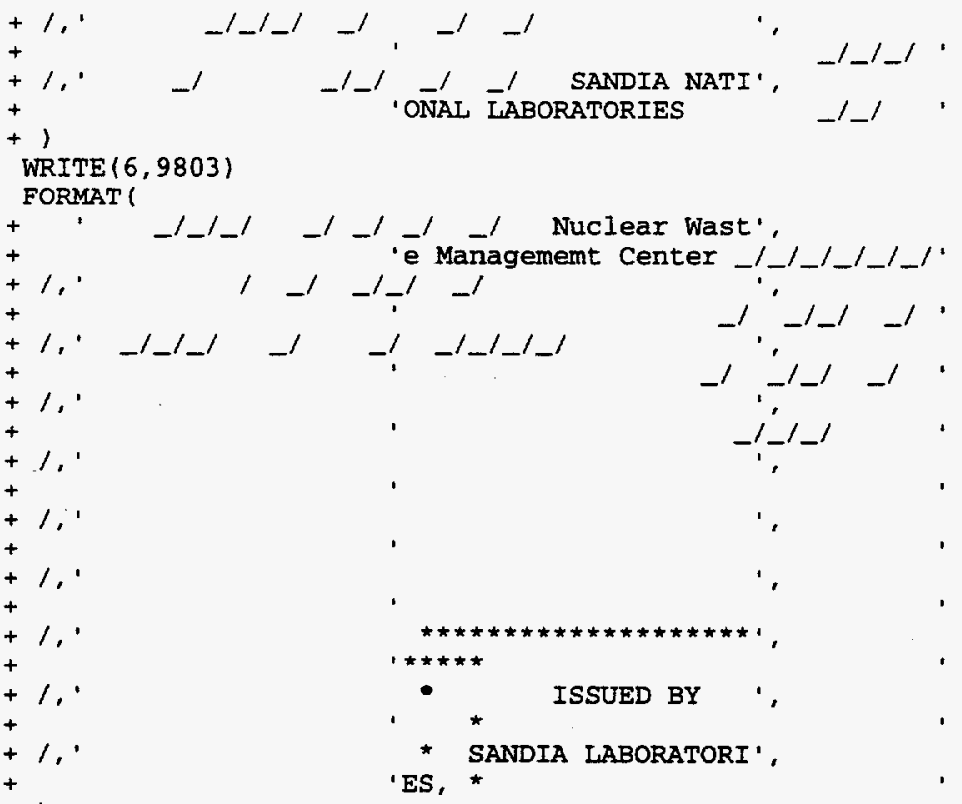

* A PRIME CONTRACT'.

1,

$1, \cdot$

1,

1,

1.'

$1, \cdot$

1

(1)

+1,

1.

WRITE $(6,9805)$

NOR ANY OF THEIR ', EMPLOYEES,

* NOR ANY OF THEIR CONTRACTORS, $S$ '

UBCONTRACTORS, OR THEIR

1.' EMPLOYEES, MAKES ANY WARRANTY,

EXPRESS OR IMPLIED, OR

$1, \cdot$

$1, \cdot$

1.

$1, \cdot$

$1, \cdot$

1,

1. 1

$+1$

WRITE $(6,9806)$

9806

FORMAT!

POST2281

POST 2282

POST2283

POST2284

POST 2285

POST2286

POST 2287

POST 2288

POST2289

POST 2290

POST2291

POST2 292

POST2 293

POST2294

POST 2295

POST2296

POST2297

POST2298

POST2299

POST2300

POST2301

POST2302

POST2 303

POST2304

POST2305

POST2306

POST2307

POST 2308

POST2309

POST 2310

POST2311

POST 2312

POST2313

POST2314

POST2315

POST2316

POST2317

POST2318

POST2319

POST2 320

POST2321

POST2322

POST2323

POST2324

POST2325

POST2326

POST 2327

POST2328

POST2 329

POST2330

POST2331

POST2 332

POST2333

POST2334

POST2335

POST2336

POST 2337

POST2338

POST 2339

POST 2340

POST2341

POST2342

POST 2343

POST2344

POST2345

POST2346

POST2347

POST2348

POST2 349

POST2350

POST2351

POST2352

POST 2353

POST2354

POST2355

POST2356 


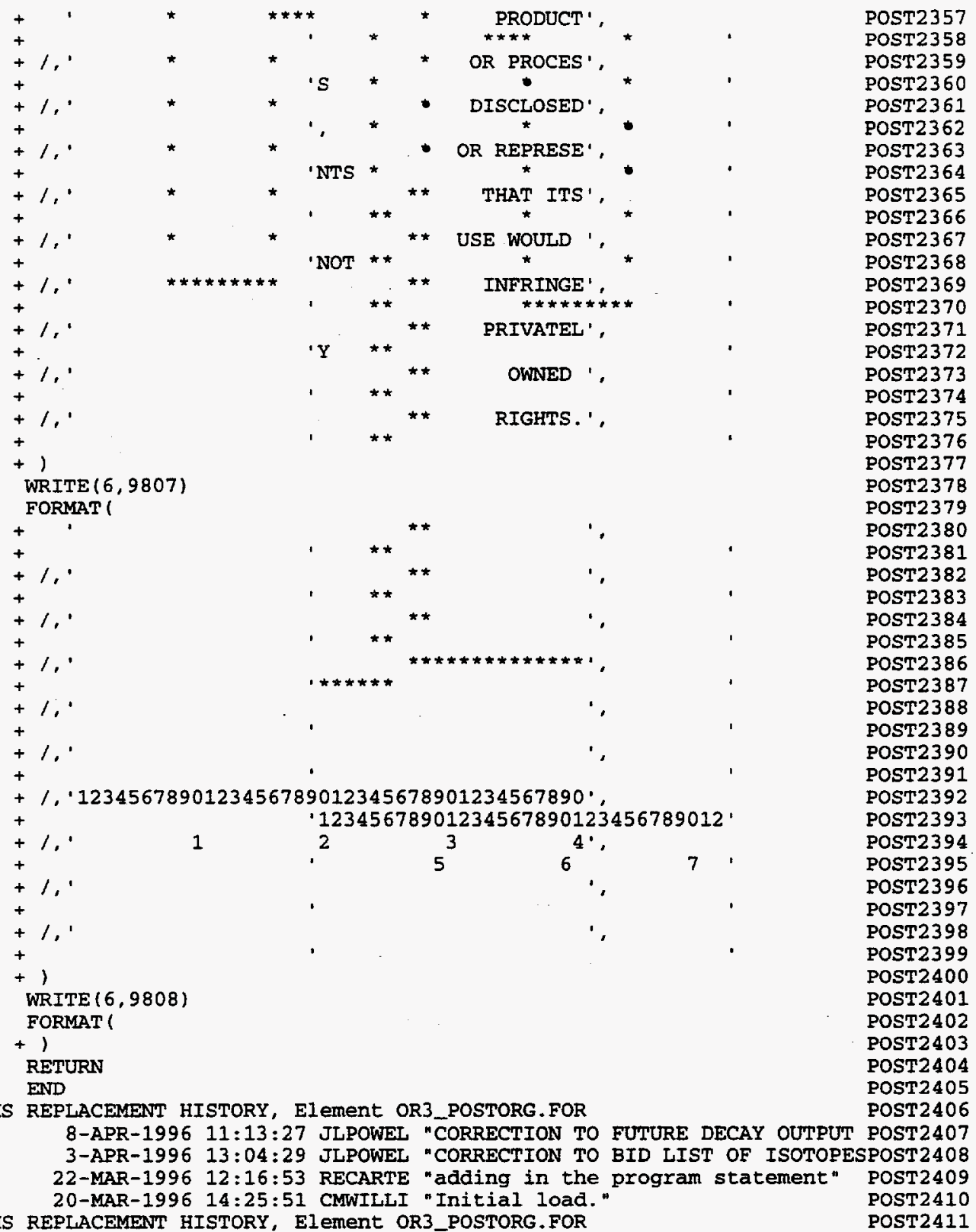


APPENDIX G: SAMPLE OUTPUT FILES FROM POSTORG 
Intentionally Left Blank 


\section{Appendix G: Sample Output Files From Postorg}

This appendix contains the three posible output files that can be generated from PostORG. The files include the following: ORNLCH93.SUM, ORNLC93F.U5, and ORNLC93F.SUM. The file ORNLCH93.SUM is a file containing the total 1993 nuclide concentrations after all initial inventories listed in ORNL__.DAT have been decayed to the base year of 1993. The file ORNLC93F.U5 is an ORIGEN2 input file to decay the nuclide concentrations in ORNLCH93.SUM 75 years beyond the base year of 1993. Finally, ORNLC93F.SUM is the processed and sorted ORIGEN2 output for ORNLC93F.U5. 


\section{ornlch93.sum FILE LISTING}

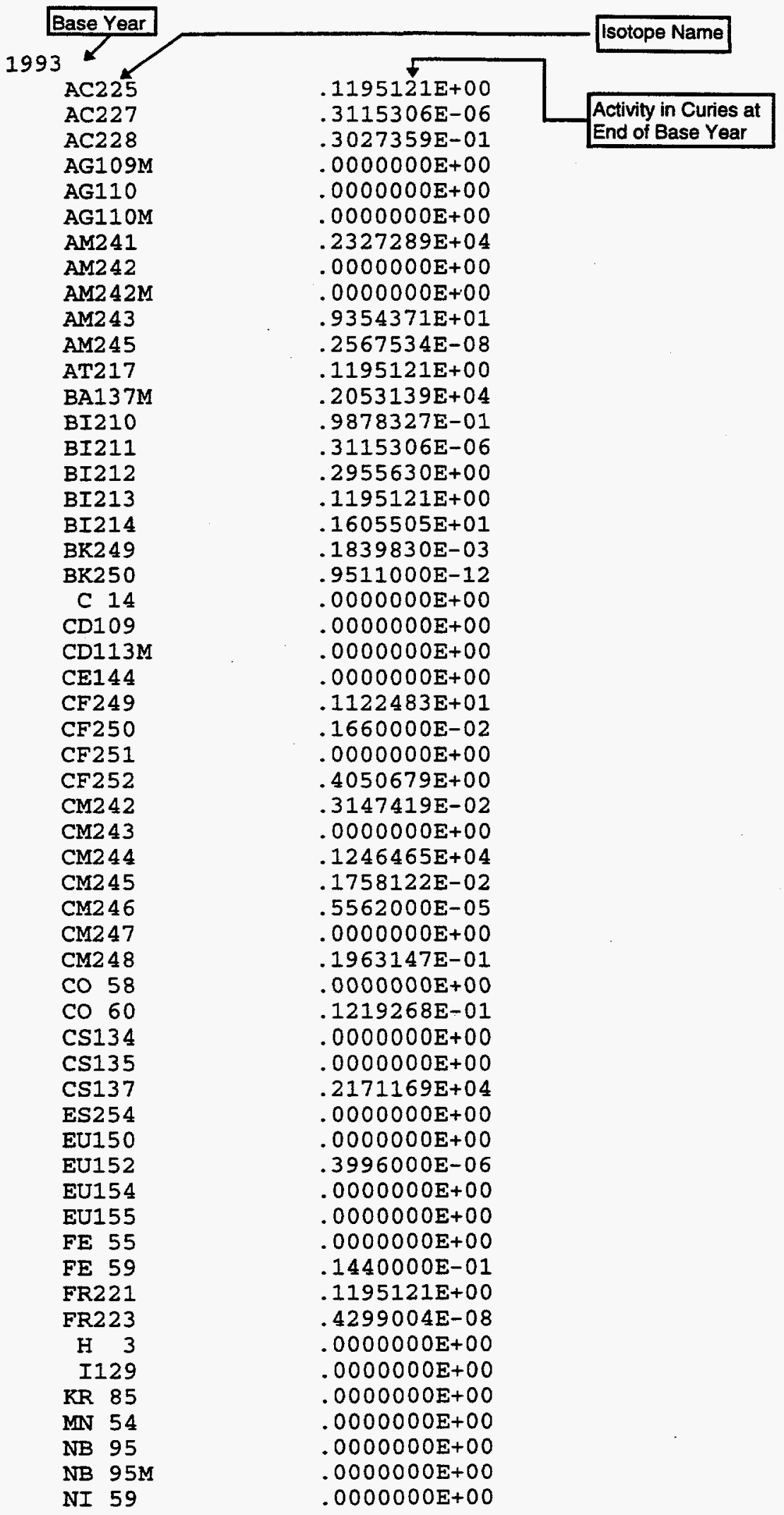


ornlch93.sum FILE LISTING

\begin{tabular}{|c|c|c|}
\hline 58 & NI 63 & $.1109000 \mathrm{E}-03$ \\
\hline 59 & NP237 & $.6030983 E+01$ \\
\hline 60 & NP2 38 & $.0000000 \mathrm{E}+00$ \\
\hline 61 & NP239 & $.9354371 E+01$ \\
\hline 62 & NP240 & $.0000000 \mathrm{E}+00$ \\
\hline 63 & NP2 $40 M$ & $.7911798 \mathrm{E}-09$ \\
\hline 64 & PA231 & $.1560855 E-05$ \\
\hline 65 & PA233 & $.6030983 E+01$ \\
\hline 66 & $\mathrm{PA} 234$ & $.3419687 \mathrm{E}-04$ \\
\hline 67 & PA234M & $.2630038 E-01$ \\
\hline 68 & PB209 & $.1195121 \mathrm{E}+00$ \\
\hline 69 & PB210 & $.9878327 \mathrm{E}-01$ \\
\hline 70 & PB211 & $.3115306 E-06$ \\
\hline 71 & PB212 & $.2955630 \mathrm{E}+00$ \\
\hline 72 & PB214 & $.1605505 E+01$ \\
\hline 73 & PD107 & $.0000000 \mathrm{E}+00$ \\
\hline 74 & PM147 & $.1454935 \mathrm{E}-01$ \\
\hline 75 & PO210 & $.7316528 \mathrm{E}-01$ \\
\hline 76 & P0211 & $.8723459 \mathrm{E}-09$ \\
\hline 77 & PO212 & $.1893680 \mathrm{E}+00$ \\
\hline 78 & PO213 & $.1169344 \mathrm{E}+00$ \\
\hline 79 & $\mathrm{PO} 214$ & $.1604504 \mathrm{E}+01$ \\
\hline 80 & $\mathrm{PO} 215$ & $.3115306 \mathrm{E}-06$ \\
\hline 81 & PO216 & $.2955630 \mathrm{E}+00$ \\
\hline 82 & PO218 & $.1605505 E+01$ \\
\hline 83 & PR144 & $.0000000 E+00$ \\
\hline 84 & PU236 & $.0000000 E+00$ \\
\hline 85 & PU238 & $.6783259 E+04$ \\
\hline 86 & PU239 & $.7955260 E+03$ \\
\hline 87 & PU240 & $.7256365 E+03$ \\
\hline 88 & PU241 & $.5527624 E+05$ \\
\hline 89 & PU242 & $.5463922 \mathrm{E}+01$ \\
\hline 90 & PU243 & $.0000000 \mathrm{E}+00$ \\
\hline 91 & PU2 44 & $.7921432 \mathrm{E}-09$ \\
\hline 92 & RA223 & $.3115306 \mathrm{E}-06$ \\
\hline 93 & RA224 & $.2955630 \mathrm{E}+00$ \\
\hline 94 & RA225 & $.1195121 \mathrm{E}+00$ \\
\hline 95 & RA226 & $.1605505 \mathrm{E}+01$ \\
\hline 96 & RA228 & $.3027359 \mathrm{E}-01$ \\
\hline 97 & $\mathrm{RH} 106$ & $.0000000 E+00$ \\
\hline 98 & RN219 & $.3115306 \mathrm{E}-06$ \\
\hline 99 & RN220 & $.2955630 \mathrm{E}+00$ \\
\hline 100 & $\mathrm{RN} 222$ & $.1605505 \mathrm{E}+01$ \\
\hline 101 & RU106 & $.0000000 E+00$ \\
\hline 102 & SB125 & $.0000000 E+00$ \\
\hline 103 & SB126 & $.0000000 E+00$ \\
\hline 104 & SB126M & $.0000000 E+00$ \\
\hline 105 & SE 79 & $.0000000 \mathrm{E}+00$ \\
\hline 106 & SM151 & $.0000000 E+00$ \\
\hline 107 & SN119M & $.0000000 \mathrm{E}+00$ \\
\hline 108 & SN121M & $.0000000 E+00$ \\
\hline 109 & SN126 & $.0000000 \mathrm{E}+00$ \\
\hline 110 & SR 90 & $.1289080 \mathrm{E}+04$ \\
\hline 111 & TC 99 & $.1779000 \mathrm{E}+02$ \\
\hline 112 & TE125M & $.0000000 \mathrm{E}+00$ \\
\hline 113 & TE127 & $.0000000 E+00$ \\
\hline 114 & TE127M & $.0000000 \mathrm{E}+00$ \\
\hline
\end{tabular}




$$
\pi
$$


ornic93f.u5 FiLE LISTING

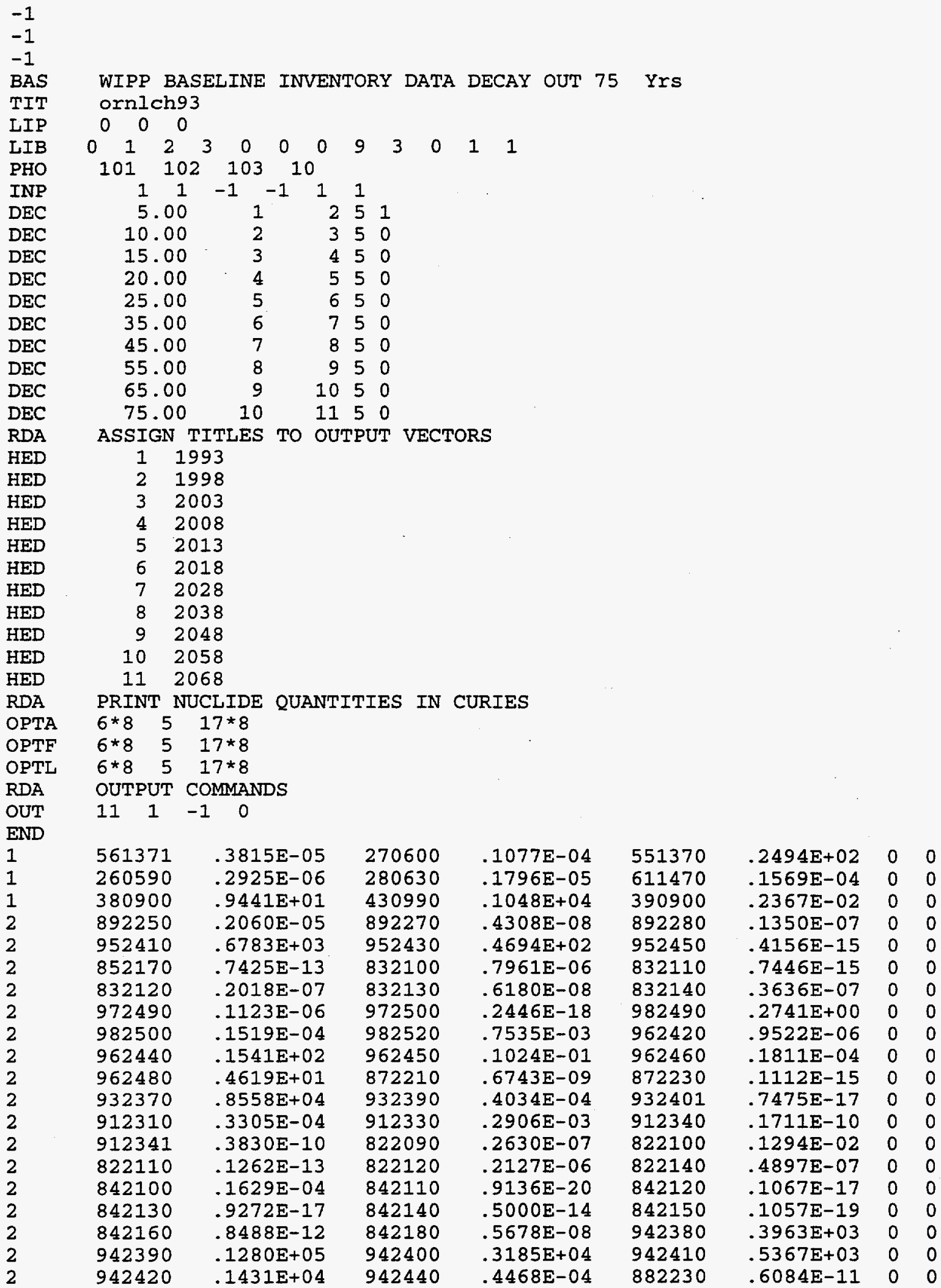




\section{Appendix G: Sample Output Files From PostORG}

ornlc93f.u5 FILE LISTING

\begin{tabular}{|c|c|c|c|c|c|c|c|c|}
\hline 59 & 2 & 882240 & $.1856 \mathrm{E}-05$ & 882250 & $.3049 E-05$ & 882260 & $.1624 \mathrm{E}+01$ & 0 \\
\hline 60 & 2 & 882280 & $.1294 \mathrm{E}-03$ & 862190 & $.2395 E-16$ & 862200 & $.3205 E-09$ & 0 \\
\hline 61 & 2 & 862220 & $.1044 \mathrm{E}-04$ & 902270 & $.9997 \mathrm{E}-11$ & 902280 & $.3607 \mathrm{E}-03$ & 0 \\
\hline 62 & 2 & 902290 & $.5619 \mathrm{E}+00$ & 902300 & $.3233 E-01$ & 902310 & $.1145 \mathrm{E}-07$ & 0 \\
\hline 63 & 2 & 902320 & $.3125 E+06$ & 902340 & $.1136 \mathrm{E}-05$ & 812070 & $.1631 \mathrm{E}-14$ & 0 \\
\hline 64 & 2 & 812080 & $.3606 E-09$ & 812090 & $.5681 E-09$ & 922320 & $.1348 E-01$ & 0 \\
\hline 65 & 2 & 922330 & $.9231 E+04$ & 922340 & $.1425 E+04$ & 922350 & $.3039 E+04$ & 0 \\
\hline 66 & 2 & 922360 & $.3764 E+01$ & 922370 & $.1575 E-04$ & 922380 & $.1052 \mathrm{E}+06$ & 0 \\
\hline 67 & 2 & 922400 & $.8545 E-15$ & 0 & & & & \\
\hline 68 & 3 & 631520 & $.2310 E-08$ & 0 & & & & \\
\hline
\end{tabular}


ornic93f.u5 FILE LISTING

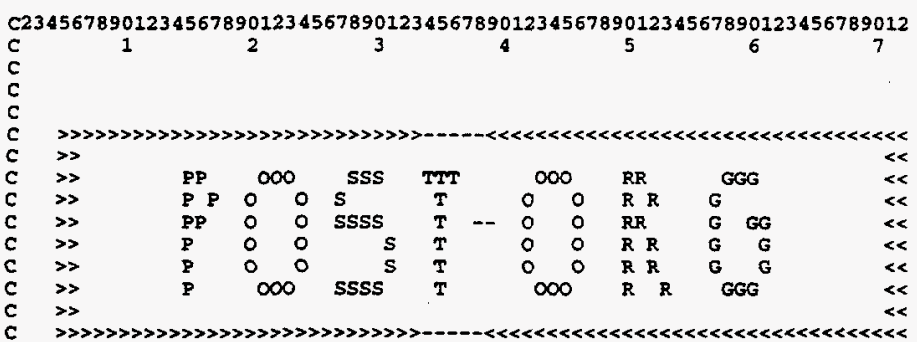

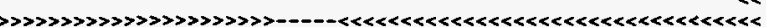
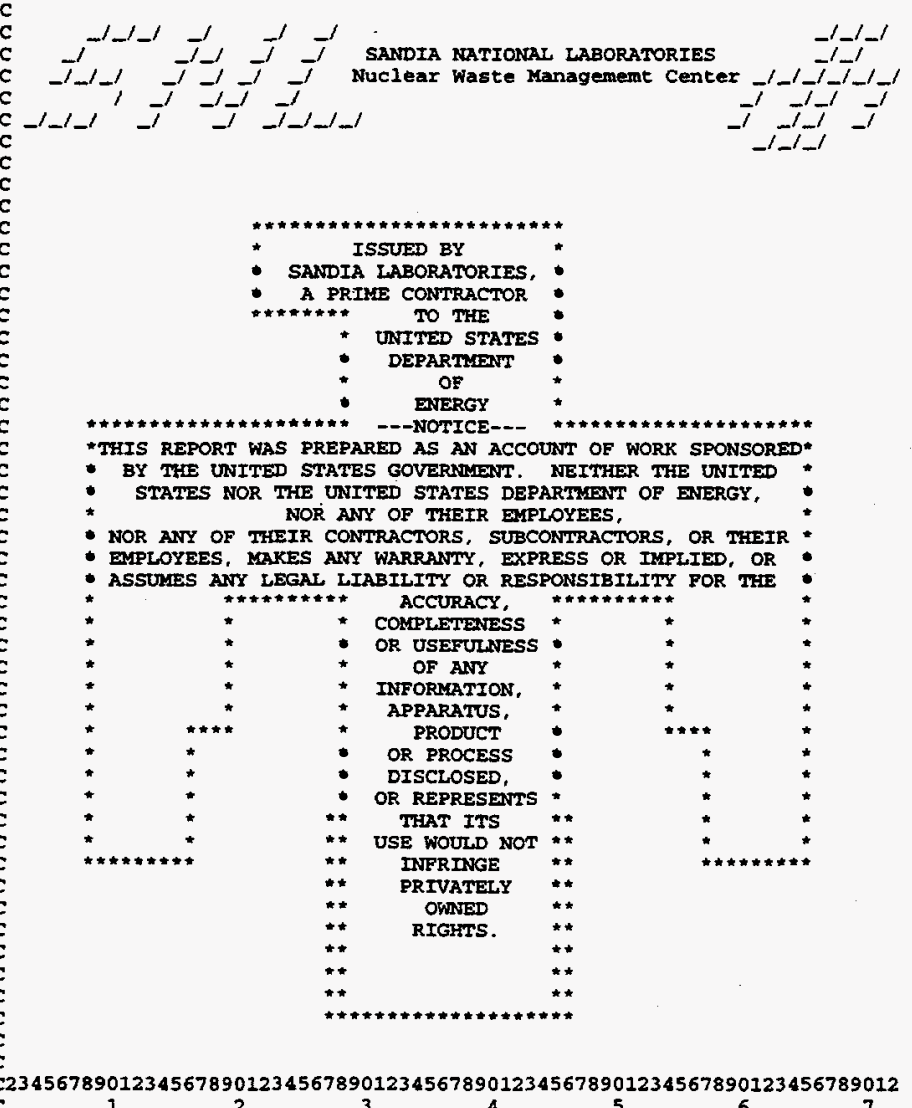

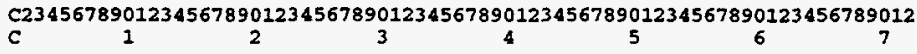

THIS PROGRAM WILL PROCESS AN ORIGEN2 OUTPUT FILE

GENERATED FROM USING PYEORG. FOR - IT WILL SUM ALI

NUCLIDE CONCENTRATIONS TO USE TO CREATE A NEW ORIG

INPUT FILE TO DECAY THE DATA INTO THE FUTURE

PLEASE ENTER ORIGEN2 OUTPUT FIILNAME TO BE

PROCESSED WITHOUT EXTENSION (i.e. Ornlch95 or

ornle95a). THE FILENAME MUST BE B CHARACTERS

LONG, NOT INCLUDING THE EXTENSION . 26 :

\section{ornlch95.u6}

ENTER BASE YEAR INITIALCY DECAYED ALT INVENTORY DA TO PRIOR TO DECAYING INTO FUTURE (i.e. 1993, 1995) MAKE SURE THIS IS THE SAME YEAR USED WHILE RUNNING PreORG. for 


\section{ornic93f.u5 FILE LISTING}

WOULD YOU LIKE TO CREATE AN ORIGEN2 TNPUT FILE

TO DECAY 1995 INVINTORY DATA INTO FUTURE? $y$ (YES) or $n$ (NO)

\section{$y \longleftarrow$ USER ANSWER}

PLEASE CHOOSE HOW LONG INRO FUTURE YOU WISH TO DECAY INVENTORY OUT TO:
(a) 75 yxs.
(b) $100 \mathrm{yrs}$
(c) $500 \mathrm{yrs}$

(d) 1000 yrs.

(e) 5000 yrs.

(f) 10,000 yrs.

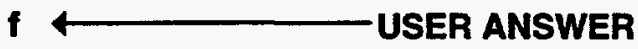

ELIMINATING EXCESS DATA FROM ORIGEN2 OUTPUT CREATING OUTPUT FILES FOR EACH YEAR OF DATA

GENERATING MASTER LIST OF NUCLIDES FOR SITE DATA

ALPHABETIZING MASTER IIST OF NUCLIDES

SUMAING NUCLIDE CONCENTRATIONS

SORTING INTO WIPP BIR REV.1 Appendix E NUCLIDE LIST INPUT FILENAME = OInleh95. SUM

WIPP BIR Appendix E Nuclide List File: ornlch95.bid

GENERATING ORIGEN2 INPUT FILE TO DECAY BASE YEAR DATA INTO FUTURE

ORIGEN2 input filename = ornle95f.u5

TO RUN PROGRAM AGAIN ENTER 1

TO EXIT PROGRAM ENTER 2: 

3.117E-07 5.483E-07 $8.524 \mathrm{E}-07 \quad 1.214 \mathrm{E}-06 \quad 1.625 \mathrm{E}-06 \quad 2.078 \mathrm{E}-06 \quad 3.087 \mathrm{E}-06 \quad 4.201 \mathrm{E}-06 \quad 5.392 \mathrm{E}-06 \quad 6.640 \mathrm{E}-06 \quad 7.929 \mathrm{E}-06$ 3.028E-02 3.191E-02 3.287E-02 3.344E-02 3.378E-02 3.399E-02 3.418E-02 3.425E-02 $3.427 \mathrm{E}-02 \quad 3.428 \mathrm{E}-02 \quad 3.429 \mathrm{E}-02$ $\begin{array}{llllllllllll}0.000 \mathrm{E}+00 & 0.000 \mathrm{E}+00 & 0.000 \mathrm{E}+00 & 0.000 \mathrm{E}+00 & 0.000 \mathrm{E}+00 & 0.000 \mathrm{E}+00 & 0.000 \mathrm{E}+00 & 0.000 \mathrm{E}+00 & 0.000 \mathrm{E}+00 & 0.000 \mathrm{E}+00 & 0.000 \mathrm{E}+00\end{array}$ $\begin{array}{lllllllllllll}0.000 E+00 & 0.000 E+00 & 0.000 E+00 & 0.000 E+00 & 0.000 E+00 & 0.000 E+00 & 0.000 E+00 & 0.000 E+00 & 0.000 E+00 & 0.000 E+00 & 0.000 E+00\end{array}$ $0.000 E+00 \quad 0.000 E+00 \quad 0.000 E+00 \quad 0.000 E+00 \quad 0.000 E+00 \quad 0.000 E+00 \quad 0.000 E+000.000 E+00 \quad 0.000 E+000.000 E+00 \quad 0.000 E+00$

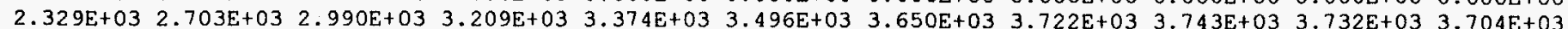
$0.000 \mathrm{E}+00 \quad 0.000 \mathrm{E}+000.000 \mathrm{E}+00 \quad 0.000 \mathrm{E}+00 \quad 0.000 \mathrm{E}+00 \quad 0.000 \mathrm{E}+00 \quad 0.000 \mathrm{E}+00 \quad 0.000 \mathrm{E}+000.000 \mathrm{O}+00 \quad 0.000 \mathrm{E}+000.000 \mathrm{E}+00$

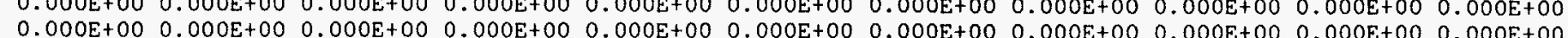

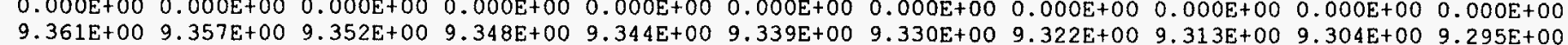
$\begin{array}{lllllllllll}9.361 \mathrm{E}+00 & 9.357 \mathrm{E}+00 & 9.352 \mathrm{E}+00 & 9.348 \mathrm{E}+00 & 9.344 \mathrm{E}+00 & 9.339 \mathrm{E}+00 & 9.330 \mathrm{E}+00 & 9.322 \mathrm{E}+00 & 9.313 \mathrm{E}+00 & 9.304 \mathrm{E}+00 & 9.295 \mathrm{E}+00 \\ 2.569 \mathrm{E}-09 & 5.113 \mathrm{E}-11 & 9.790 \mathrm{E}-13 & 1.875 \mathrm{E}-14 & 3.590 \mathrm{E}-16 & 6.874 \mathrm{E}-18 & 2.520 \mathrm{E}-21 & 9.242 \mathrm{E}-25 & 3.389 \mathrm{E}-28 & 1.242 \mathrm{E}-31 & 4.457 \mathrm{E}-35\end{array}$ $\begin{array}{lllllllllll}1.196 \mathrm{E}-01 & 1.617 \mathrm{E}-01 & 2.039 \mathrm{E}-01 & 2.460 \mathrm{E}-01 & 2.880 \mathrm{E}-01 & 3.301 \mathrm{E}-01 & 4.142 \mathrm{E}-01 & 4.982 \mathrm{E}-01 & 5.821 \mathrm{E}-01 & 6.659 \mathrm{E}-01 & 7.496 \mathrm{E}-01\end{array}$ $\begin{array}{lllllllllll}2.053 \mathrm{E}+03 & 1.829 \mathrm{E}+03 & 1.630 \mathrm{E}+03 & 1.452 \mathrm{E}+03 & 1.293 \mathrm{E}+03 & 1.152 \mathrm{E}+03 & 9.145 \mathrm{E}+02 & 7.259 \mathrm{E}+02 & 5.761 \mathrm{E}+02 & 4.573 \mathrm{E}+02 & 3.629 \mathrm{E}+02\end{array}$ $\begin{array}{lllllllllll}9.881 \mathrm{E}-02 & 3.155 \mathrm{E}-01 & 5.005 \mathrm{E}-01 & 6.584 \mathrm{E}-01 & 7.931 \mathrm{E}-01 & 9.079 \mathrm{E}-01 & 1.089 \mathrm{E}+00 & 1.220 \mathrm{E}+00 & 1.314 \mathrm{E}+00 & 1.381 \mathrm{E}+00 & 1.428 \mathrm{E}+00\end{array}$

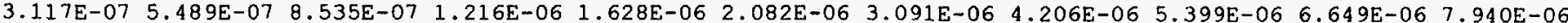

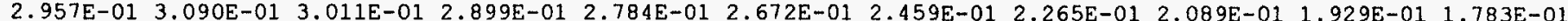
(. $1.60 \mathrm{E}+00$ 1. 1. $1.841 \mathrm{E}-04$ 3.525E-06 $6.750 \mathrm{E}-081.293 \mathrm{E}$

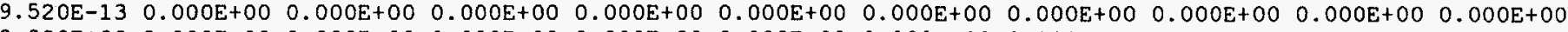
$\begin{array}{lllllllllll}0.000 \mathrm{E}+00 & 0.000 \mathrm{E}+00 & 0.000 \mathrm{E}+00 & 0.000 \mathrm{E}+00 & 0.000 \mathrm{E}+00 & 0.000 \mathrm{E}+00 & 0.000 \mathrm{E}+00 & 0.000 \mathrm{E}+00 & 0.000 \mathrm{E}+00 & 0.000 \mathrm{E}+00 & 0.000 \mathrm{E}+00\end{array}$ $\begin{array}{llllllllllll}0.000 \mathrm{E}+00 & 0.000 \mathrm{E}+00 & 0.000 \mathrm{E}+00 & 0.000 \mathrm{E}+00 & 0.000 \mathrm{E}+00 & 0.000 \mathrm{E}+00 & 0.000 \mathrm{E}+00 & 0.000 \mathrm{E}+00 & 0.000 \mathrm{E}+00 & 0.000 \mathrm{E}+00 & 0.000 \mathrm{E}+00\end{array}$ $\begin{array}{lllllllllllll}0.000 \mathrm{E}+00 & 0.000 \mathrm{E}+00 & 0.000 \mathrm{E}+00 & 0.000 \mathrm{E}+00 & 0.000 \mathrm{E}+00 & 0.000 \mathrm{E}+00 & 0.000 \mathrm{E}+00 & 0.000 \mathrm{E}+00 & 0.000 \mathrm{E}+00 & 0.000 \mathrm{E}+00 & 0.000 \mathrm{E}+00\end{array}$ $\begin{array}{llllllllllllll}0.000 \mathrm{E}+00 & 0.000 \mathrm{E}+00 & 0.000 \mathrm{E}+00 & 0.000 \mathrm{E}+00 & 0.000 \mathrm{E}+00 & 0.000 \mathrm{E}+00 & 0.000 \mathrm{E}+00 & 0.000 \mathrm{E}+00 & 0.000 \mathrm{E}+00 & 0.000 \mathrm{E}+00 & 0.000 \mathrm{E}+00\end{array}$

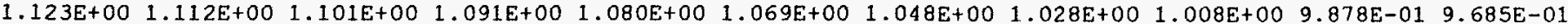

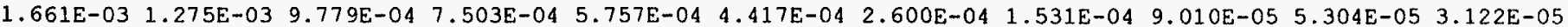
$0.000 \mathrm{E}+00 \quad 0.000 \mathrm{E}+00 \quad 0.000 \mathrm{E}+00 \quad 0.000 \mathrm{E}+00 \quad 0.000 \mathrm{E}+00 \quad 0.000 \mathrm{E}+00 \quad 0.000 \mathrm{E}+00 \quad 0.000 \mathrm{E}+00 \quad 0.000 \mathrm{E}+00 \quad 0.000 \mathrm{E}+000.000 \mathrm{E}+00$

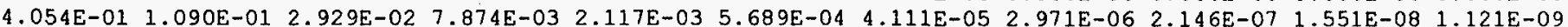
$3.150 \mathrm{E}-03 \quad 1.348 \mathrm{E}-06 \quad 5.765 \mathrm{E}-10 \quad 2.467 \mathrm{E}-13 \quad 1.055 \mathrm{E}-16 \quad 4.515 \mathrm{E}-20 \quad 8.265 \mathrm{E}-27 \quad 1.513 \mathrm{E}-33 \quad 0.000 \mathrm{E}+00 \quad 0.000 \mathrm{E}+000.000 \mathrm{E}+00$

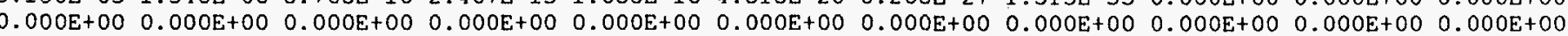

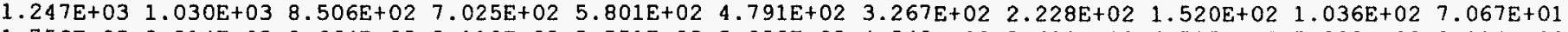

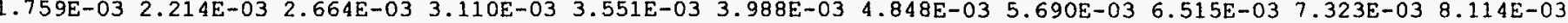

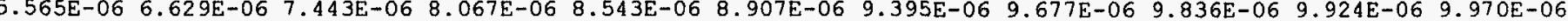
$\begin{array}{llllllllllll}0.000 \mathrm{E}+00 & 0.000 \mathrm{E}+00 & 0.000 \mathrm{E}+00 & 0.000 \mathrm{E}+00 & 0.000 \mathrm{E}+00 & 0.000 \mathrm{E}+00 & 0.000 \mathrm{E}+00 & 0.000 \mathrm{E}+00 & 0.000 \mathrm{E}+00 & 0.000 \mathrm{E}+00 & 0.000 \mathrm{E}+00\end{array}$ 1.965E-02 1.965E-02 1.965E-02 1.965E-02 $1.965 \mathrm{E}-02 \quad 1.965 \mathrm{E}-02 \quad 1.965 \mathrm{E}-02 \quad 1.965 \mathrm{E}-02 \quad 1.965 \mathrm{E}-02 \quad 1.965 \mathrm{E}-02 \quad 1.965 \mathrm{E}-02$ $0.000 E+00 \quad 0.000 E+00 \quad 0.000 E+00 \quad 0.000 E+00 \quad 0.000 E+00 \quad 0.000 E+00 \quad 0.000 E+00 \quad 0.000 E+00 \quad 0.000 E+000.000 E+00 \quad 0.000 E+00$

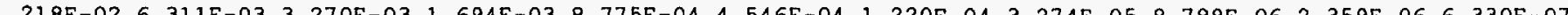
$0.000 E+000.000 E+000.000 E+000.000 E+00 \quad 0.000 E+000.000 E+00 \quad 0.000 E+000.000 E+000.000 E+00 \quad 0.000 E+000.000 E+00$ $0.000 E+000.000 E+000.00 E+000.00 E+00 \quad 0.000 E+000.00 E+00 \quad 0.000 E+00 \quad 0.000 E+00 \quad 0.000 E+000.000 E+00 \quad 0.000 E+00$ $\begin{array}{lllllllllll}0.000 E+00 & 0.000 E+00 & 0.000 E+00 & 0.000 E+00 & 0.000 E+00 & 0.000 E+00 & 0.000 E+00 & 0.000 E+00 & 0.000 E+00 & 0.000 E+00 & 0.000 E+00 \\ 2.170 E+03 & 1.934 E+03 & 1.723 E+03 & 1.535 E+03 & 1.367 E+03 & 1.218 E+03 & 9.667 E+02 & 7.673 E+02 & 6.090 E+02 & 4.834 E+02 & 3.836 E+02\end{array}$ $\begin{array}{lllllllllllll}0.000 \mathrm{E}+00 & 0.000 \mathrm{E}+00 & 0.000 \mathrm{E}+00 & 0.000 \mathrm{E}+00 & 0.000 \mathrm{E}+00 & 0.000 \mathrm{E}+00 & 0.000 \mathrm{E}+00 & 0.000 \mathrm{E}+00 & 0.000 \mathrm{E}+00 & 0.000 \mathrm{E}+00 & 0.000 \mathrm{E}+00\end{array}$ $\begin{array}{llllllllllll}0.000 \mathrm{E}+00 & 0.000 \mathrm{E}+00 & 0.000 \mathrm{E}+00 & 0.000 \mathrm{E}+00 & 0.000 \mathrm{E}+00 & 0.000 \mathrm{E}+00 & 0.000 \mathrm{E}+00 & 0.000 \mathrm{E}+00 & 0.000 \mathrm{E}+00 & 0.000 \mathrm{E}+00 & 0.000 \mathrm{E}+00\end{array}$

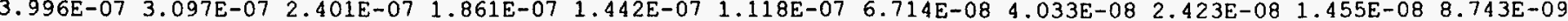
$\begin{array}{lllllllllllll}0.000 \mathrm{E}+00 & 0.000 \mathrm{E}+00 & 0.000 \mathrm{E}+00 & 0.000 \mathrm{E}+00 & 0.000 \mathrm{E}+00 & 0.000 \mathrm{E}+00 & 0.000 \mathrm{E}+00 & 0.000 \mathrm{E}+00 & 0.000 \mathrm{E}+00 & 0.000 \mathrm{E}+00 & 0.000 \mathrm{E}+00\end{array}$ $\begin{array}{llllllllllll}0.000 E+00 & 0.000 E+00 & 0.000 E+00 & 0.000 E+00 & 0.000 E+00 & 0.000 E+00 & 0.000 E+00 & 0.000 E+00 & 0.000 E+00 & 0.000 E+00 & 0.000 E+00\end{array}$ $0.000+00 \quad 0.000 E+000.000 E+000.000 E+00 \quad 0.000 E+000.000 E+000.000 E+000.000 E+000.000 E+000.000 E+00 \quad 0.000 E+00$ $\begin{array}{lllllllllllll}0.000 E+00 & 0.000 E+00 & 0.000 E+00 & 0.000 E+00 & 0.000 E+00 & 0.000 E+00 & 0.000 E+00 & 0.000 E+00 & 0.000 E+00 & 0.000 E+00 & 0.000 E+00\end{array}$ $\begin{array}{llllllllllll}1.196 \mathrm{E}-01 & 1.617 \mathrm{E}-01 & 2.039 \mathrm{E}-01 & 2.460 \mathrm{E}-01 & 2.880 \mathrm{E}-01 & 3.301 \mathrm{E}-01 & 4.142 \mathrm{E}-01 & 4.982 \mathrm{E}-01 & 5.821 \mathrm{E}-01 & 6.659 \mathrm{E}-01 & 7.496 \mathrm{E}-01\end{array}$

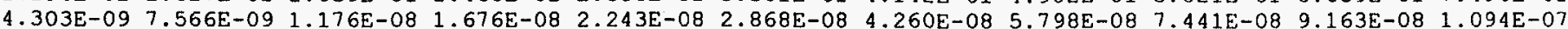
$\begin{array}{lllllllllll}0.000 E+00 & 0.000 E+00 & 0.000 E+00 & 0.000 E+00 & 0.000 E+00 & 0.000 E+00 & 0.000 E+00 & 0.000 E+00 & 0.000 E+00 & 0.000 E+00 & 0.000 E+00\end{array}$ $\begin{array}{llllllllllllll}0.000 E+00 & 0.000 E+00 & 0.000 E+00 & 0.000 E+00 & 0.000 E+00 & 0.000 E+00 & 0.000 E+00 & 0.000 E+00 & 0.000 E+00 & 0.000 E+00 & 0.000 E+00\end{array}$ $\begin{array}{lllllllllllll}0.000 \mathrm{E}+00 & 0.000 \mathrm{E}+00 & 0.000 \mathrm{E}+00 & 0.000 \mathrm{E}+00 & 0.000 \mathrm{E}+00 & 0.000 \mathrm{E}+00 & 0.000 \mathrm{E}+00 & 0.000 \mathrm{E}+00 & 0.000 \mathrm{E}+00 & 0.000 \mathrm{E}+00 & 0.000 \mathrm{E}+00\end{array}$ $\begin{array}{llllllllllllllll}0.000 \mathrm{E}+00 & 0.000 \mathrm{E}+00 & 0.000 \mathrm{E}+00 & 0.000 \mathrm{E}+00 & 0.000 \mathrm{E}+00 & 0.000 \mathrm{E}+00 & 0.000 \mathrm{E}+00 & 0.000 \mathrm{E}+00 & 0.000 \mathrm{E}+00 & 0.000 \mathrm{E}+00 & 0.000 \mathrm{E}+00\end{array}$ 

$\begin{array}{llllllllllll}0.000 E+00 & 0.000 E+00 & 0.000 E+00 & 0.000 E+00 & 0.000 E+00 & 0.000 E+00 & 0.000 E+00 & 0.000 E+00 & 0.000 E+00 & 0.000 E+00 & 0.000 E+00 \\ 0.000 E+00 & 0.000 E+00 & 0.000 E+00 & 0.000 E+00 & 0.000 E+00 & 0.000 E+00 & 0.000 E+00 & 0.000 E+00 & 0.000 E+00 & 0.000 E+00 & 0.000 E+00\end{array}$

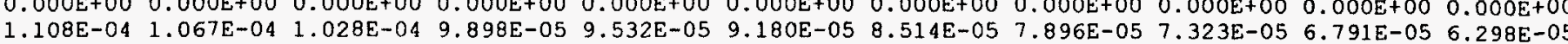

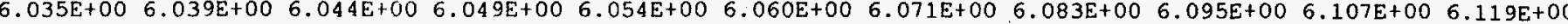
$\begin{array}{lllllllllll}0.000 \mathrm{E}+00 & 0.000 \mathrm{E}+00 & 0.000 \mathrm{E}+00 & 0.000 \mathrm{E}+00 & 0.000 \mathrm{E}+00 & 0.000 \mathrm{E}+00 & 0.000 \mathrm{E}+00 & 0.000 \mathrm{E}+00 & 0.000 \mathrm{E}+00 & 0.000 \mathrm{E}+00 & 0.000 \mathrm{E}+00\end{array}$ $\begin{array}{lllllllllll}9.361 \mathrm{E}+00 & 9.357 \mathrm{E}+00 & 9.352 \mathrm{E}+00 & 9.348 \mathrm{E}+00 & 9.344 \mathrm{E}+00 & 9.339 \mathrm{E}+00 & 9.330 \mathrm{E}+00 & 9.322 \mathrm{E}+00 & 9.313 \mathrm{E}+00 & 9.304 \mathrm{E}+00 & 9.295 \mathrm{E}+00\end{array}$

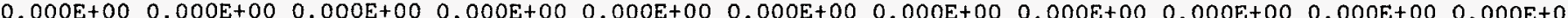

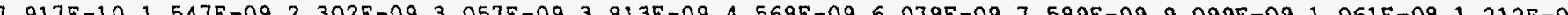

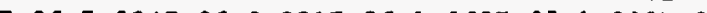

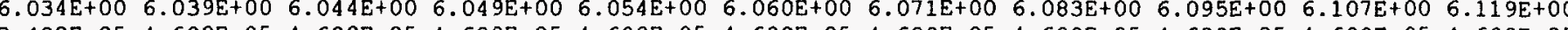

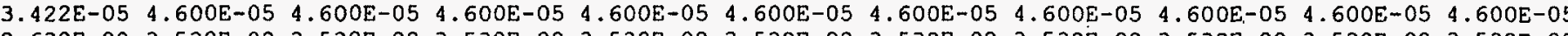
2.632E-02 3.538E-02 3.538E-02 3.538E-02 3.538E-02 $3.538 \mathrm{E}-02 \quad 3.538 \mathrm{E}-02 \quad 3.538 \mathrm{E}-02 \quad 3.538 \mathrm{E}-02 \quad 3.538 \mathrm{E}-02 \quad 3.538 \mathrm{E}-02$ $\begin{array}{lllllllllll}1.196 \mathrm{E}-01 & 1.617 \mathrm{E}-01 & 2.039 \mathrm{E}-01 & 2.460 \mathrm{E}-01 & 2.880 \mathrm{E}-01 & 3.301 \mathrm{E}-01 & 4.142 \mathrm{E}-01 & 4.982 \mathrm{E}-01 & 5.821 \mathrm{E}-01 & 6.659 \mathrm{E}-01 & 7.496 \mathrm{E}-01\end{array}$

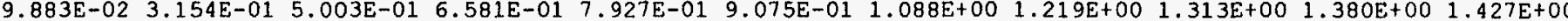
3.117E-07 5.489E-07 8.535E-07 1.216E-06 1.628E-06 2.082E-06 3.091E-06 4.206E-06 5.399E-06 $6.649 \mathrm{E}-06 \quad 7.940 \mathrm{E}-06$ $\begin{array}{llllllllllll}2.957 \mathrm{E}-01 & 3.090 \mathrm{E}-01 & 3.011 \mathrm{E}-01 & 2.899 \mathrm{E}-01 & 2.784 \mathrm{E}-01 & 2.672 \mathrm{E}-01 & 2.459 \mathrm{E}-01 & 2.265 \mathrm{E}-01 & 2.089 \mathrm{E}-01 & 1.929 \mathrm{E}-01 & 1.783 \mathrm{E}-01\end{array}$ $1.606 E+001.603 E+001.599 E+001.596 E+001.592 E+001.589 E+001.582 E+001.575 E+001.568 E+001.562 E+001.555 E+00$ $\begin{array}{lllllllllll}7.320 \mathrm{E}-02 & 3.155 \mathrm{E}-01 & 5.005 \mathrm{E}-01 & 6.585 \mathrm{E}-01 & 7.932 \mathrm{E}-01 & 9.080 \mathrm{E}-01 & 1.089 \mathrm{E}+00 & 1.220 \mathrm{E}+00 & 1.314 \mathrm{E}+00 & 1.381 \mathrm{E}+00 & 1.428 \mathrm{E}+00\end{array}$ 8.727E-10 1.537E-09 2.390E-09 3.404E-09 4.557E-09 5.828E-09 $\quad 8.654 \mathrm{E}-09 \quad 1.178 \mathrm{E}-08$ 1.512E-08 $1.862 \mathrm{E}-08 \quad 2.223 \mathrm{E}-08$ $\begin{array}{lllllllllll}1.894 \mathrm{E}-01 & 1.980 \mathrm{E}-01 & 1.929 \mathrm{E}-01 & 1.857 \mathrm{E}-01 & 1.784 \mathrm{E}-01 & 1.712 \mathrm{E}-01 & 1.575 \mathrm{E}-01 & 1.451 \mathrm{E}-01 & 1.339 \mathrm{E}-01 & 1.236 \mathrm{E}-01 & 1.143 \mathrm{E}-01\end{array}$ $\begin{array}{llllllllllll}1.170 E-01 & 1.582 \mathrm{E}-01 & 1.995 \mathrm{E}-01 & 2.406 \mathrm{E}-01 & 2.818 \mathrm{E}-01 & 3.230 \mathrm{E}-01 & 4.052 \mathrm{E}-01 & 4.874 \mathrm{E}-01 & 5.695 \mathrm{E}-01 & 6.515 \mathrm{E}-01 & 7.334 \mathrm{E}-01\end{array}$

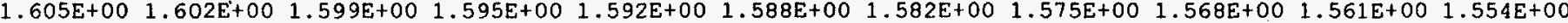

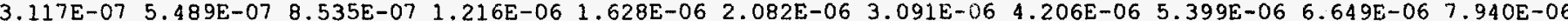

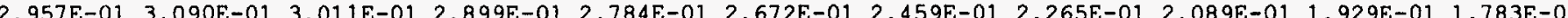
.

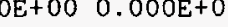
$0.000 \mathrm{E}+00 \quad 0.000 \mathrm{E}+00 \quad 0.000 \mathrm{E}+00 \quad 0.000 \mathrm{E}+00$ 0.000E+00 $0.000 \mathrm{E}+00 \quad 0.000 \mathrm{E}+00 \quad 0.000 \mathrm{E}+000.000 \mathrm{E}+00 \quad 0.000 \mathrm{E}+00 \mathrm{0} \quad 000 \mathrm{E}+00$

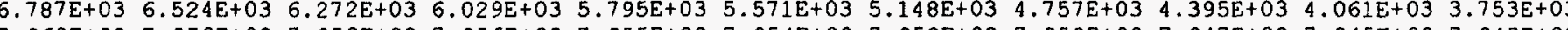

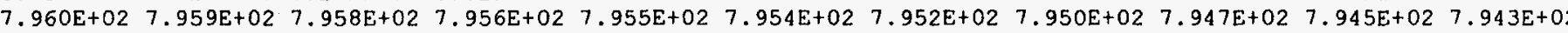

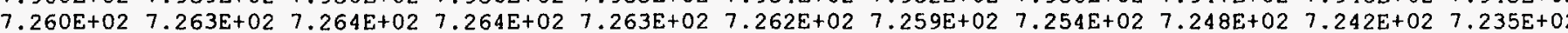
$\begin{array}{lllllllllll}5.531 \mathrm{E}+04 & 4.348 \mathrm{E}+04 & 3.418 \mathrm{E}+04 & 2.687 \mathrm{E}+04 & 2.112 \mathrm{E}+04 & 1.660 \mathrm{E}+04 & 1.026 \mathrm{E}+04 & 6.340 \mathrm{E}+03 & 3.917 \mathrm{E}+03 & 2.421 \mathrm{E}+03 & 1.496 \mathrm{E}+03\end{array}$

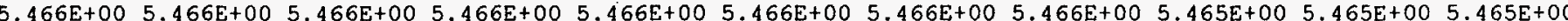
$\begin{array}{lllllllllll}0.000 E+00 & 0.000 E+00 & 0.000 E+00 & 0.000 E+00 & 0.000 E+00 & 0.000 E+00 & 0.000 E+00 & 0.000 E+00 & 0.000 E+00 & 0.000 E+00 & 0.000 E+00\end{array}$ $7.928 E-101549 E-092.305 E-093.061 E-09318 E-094574 E-096.086 E-097.598 E-092.111 E-091.062 E-081.214 E-08$ . 2.958E-01 3.090E-01 $3.011 E-012.899 E-012.784 E-012.672 E-012.459 E-012.265 E-012.089 E-011.929 E$ $\begin{array}{lllllllllll}1.196 \mathrm{E}-01 & 1.617 \mathrm{E}-01 & 2.039 \mathrm{E}-01 & 2.460 \mathrm{E}-01 & 2.880 \mathrm{E}-01 & 3.301 \mathrm{E}-01 & 4.142 \mathrm{E}-01 & 4.982 \mathrm{E}-01 & 5.821 \mathrm{E}-01 & 6.659 \mathrm{E}-01 & 7.496 \mathrm{E}-01\end{array}$

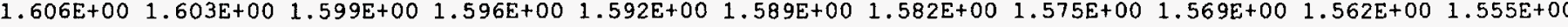
3.030E-02 3.191E-02 3.287E-02 3.344E-02 3.378E-02 3.399E-02 3.418E-02 3.425E-02 $3.427 \mathrm{E}-02 \quad 3.428 \mathrm{E}-02 \quad 3.429 \mathrm{E}-02$ $\begin{array}{lllllllllll}0.000 \mathrm{E}+00 & 0.000 \mathrm{E}+00 & 0.000 \mathrm{E}+00 & 0.000 \mathrm{E}+00 & 0.000 \mathrm{E}+00 & 0.000 \mathrm{E}+00 & 0.000 \mathrm{E}+00 & 0.000 \mathrm{E}+00 & 0.000 \mathrm{E}+00 & 0.000 \mathrm{E}+00 & 0.000 \mathrm{E}+00\end{array}$

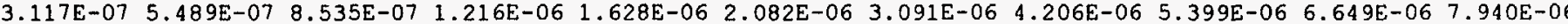
$\begin{array}{llllllllllll}2.957 E-01 & 3.090 E-01 & 3.011 E-01 & 2.899 E-01 & 2.784 E-01 & 2.672 E-01 & 2.459 E-01 & 2.265 E-01 & 2.089 E-01 & 1.929 E-01 & 1.783 E-01\end{array}$ $1.606 E+001.603 E+001.599 E+001.596 E+001.592 E+001.589 E+001.582 E+001.575 E+001.569 E+001.562 E+001.555 E+00$

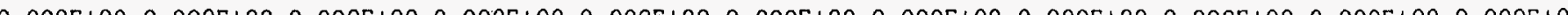

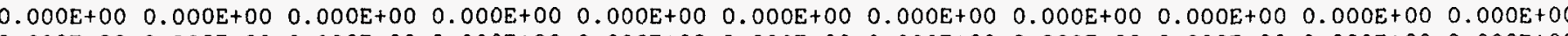
$\begin{array}{lllllllllll}0.000 E+00 & 0.000 E+00 & 0.000 E+00 & 0.000 E+00 & 0.000 E+00 & 0.000 E+00 & 0.000 E+00 & 0.000 E+00 & 0.000 E+00 & 0.000 E+00 & 0.000 E+00\end{array}$ $\begin{array}{llllllllllllllll}0.000 \mathrm{E}+00 & 0.000 \mathrm{E}+00 & 0.000 \mathrm{E}+00 & 0.000 \mathrm{E}+00 & 0.000 \mathrm{E}+00 & 0.000 \mathrm{E}+00 & 0.000 \mathrm{E}+00 & 0.000 \mathrm{E}+00 & 0.000 \mathrm{E}+00 & 0.000 \mathrm{E}+00 & 0.000 \mathrm{E}+00\end{array}$ $\begin{array}{lllllllllll}0.000 \mathrm{E}+00 & 0.000 \mathrm{E}+00 & 0.000 \mathrm{E}+00 & 0.000 \mathrm{E}+00 & 0.000 \mathrm{E}+00 & 0.000 \mathrm{E}+00 & 0.000 \mathrm{E}+00 & 0.000 \mathrm{E}+00 & 0.000 \mathrm{E}+00 & 0.000 \mathrm{E}+00 & 0.000 \mathrm{E}+00\end{array}$ $\begin{array}{lllllllllllll}0.000 \mathrm{E}+00 & 0.000 \mathrm{E}+00 & 0.000 \mathrm{E}+00 & 0.000 \mathrm{E}+00 & 0.000 \mathrm{E}+00 & 0.000 \mathrm{E}+00 & 0.000 \mathrm{E}+00 & 0.000 \mathrm{E}+00 & 0.000 \mathrm{E}+00 & 0.000 \mathrm{E}+00 & 0.000 \mathrm{E}+00\end{array}$ $\begin{array}{lllllllllllll}0.000 E+00 & 0.000 E+00 & 0.000 E+00 & 0.000 E+00 & 0.000 E+00 & 0.000 E+00 & 0.000 E+00 & 0.000 E+00 & 0.000 E+00 & 0.000 E+00 & 0.000 E+00\end{array}$ 
$\begin{array}{llllllllllllllllllllll}0.000 E+00 & 0.000 E+00 & 0.000 E+00 & 0.000 E+00 & 0.000 E+00 & 0.000 E+00 & 0.000 E+00 & 0.000 E+00 & 0.000 E+00 & 0.000 E+00 & 0.000 E+00\end{array}$

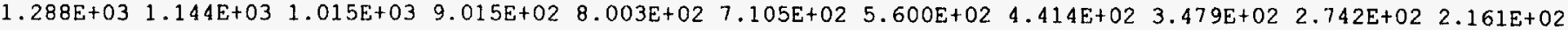
$\begin{array}{lllllllllll}1.777 E+01 & 1.777 E+01 & 1.777 E+01 & 1.777 E+01 & 1.777 E+01 & 1.777 E+01 & 1.777 E+01 & 1.777 E+01 & 1.777 E+01 & 1.777 E+01 & 1.777 E+01\end{array}$

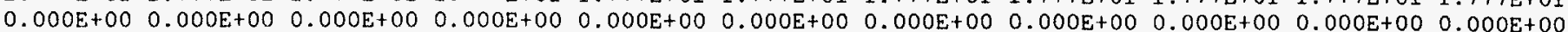

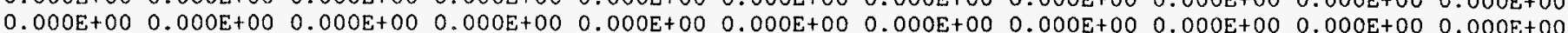

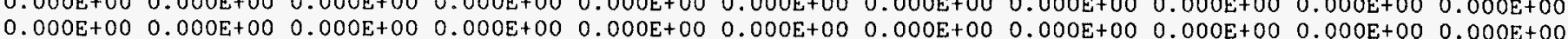

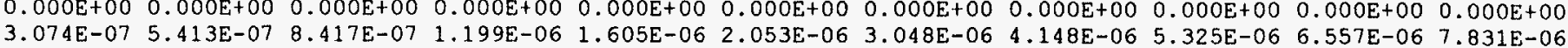
$\begin{array}{lllllllllll}2.958 E-01 & 3.087 \mathrm{E}-01 & 3.008 \mathrm{E}-01 & 2.896 \mathrm{E}-01 & 2.781 \mathrm{E}-01 & 2.669 \mathrm{E}-01 & 2.458 \mathrm{E}-01 & 2.265 \mathrm{E}-01 & 2.089 \mathrm{E}-01 & 1.929 \mathrm{E}-01 & 1.783 \mathrm{E}-01\end{array}$

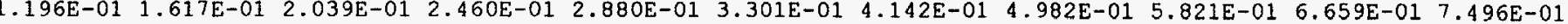

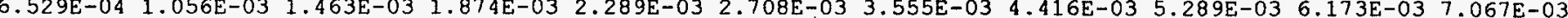

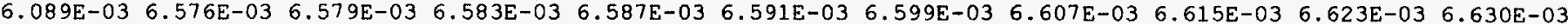
3. $429 \mathrm{E}-02$ 3. $429 \mathrm{E}-02 \quad 3.429 \mathrm{E}-02 \quad 3.429 \mathrm{E}-02 \quad 3.429 \mathrm{E}-02 \quad 3.429 \mathrm{E}-02 \quad 3.429 \mathrm{E}-02 \quad 3.429 \mathrm{E}-02 \quad 3.429 \mathrm{E}-02 \quad 3.429 \mathrm{E}-02 \quad 3.429 \mathrm{E}-02$ 2.632E-02 3.538E-02 3.538E-02 3.538E-02 3.5385-02 3.538E-02 3.538E-02 3.538E-02 3.538E-02 3.530E-02 $3.5380-02$

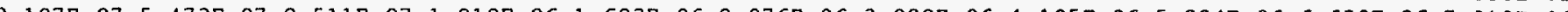
(.

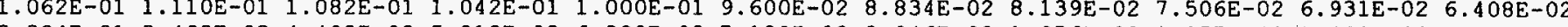

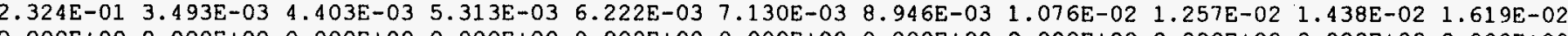
$\begin{array}{lllllllllllllllllll}0.000 \mathrm{E}+00 & 0.000 \mathrm{E}+00 & 0.000 \mathrm{E}+00 & 0.000 \mathrm{E}+00 & 0.000 \mathrm{E}+00 & 0.000 \mathrm{E}+00 & 0.000 \mathrm{E}+00 & 0.000 \mathrm{E}+00 & 0.000 \mathrm{E}+00 & 0.000 \mathrm{E}+00 & 0.000 \mathrm{E}+00\end{array}$ $\begin{array}{lllllllllll}2.886 \mathrm{E}-01 & 2.751 \mathrm{E}-01 & 2.621 \mathrm{E}-01 & 2.498 \mathrm{E}-01 & 2.381 \mathrm{E}-01 & 2.269 \mathrm{E}-01 & 2.061 \mathrm{E}-01 & 1.872 \mathrm{E}-01 & 1.700 \mathrm{E}-01 & 1.544 \mathrm{E}-01 & 1.402 \mathrm{E}-01\end{array}$

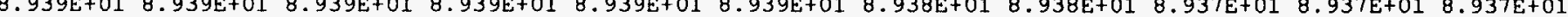

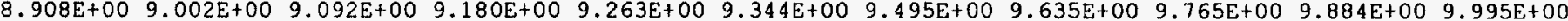
6.572E-03 6.576E-03 6.579E-03 6.583E-03 6.587E-03 6.591E-03 6.599E-03 6.607E-03 6. 615E-03 6.623E-03 $6.630 \mathrm{E}-03$ $2.436 \mathrm{E}-04$ 3.511E-04 4. 586E-04 5.661E-04 $6.736 \mathrm{E}-04$ 7.811E-04 $9.961 \mathrm{E}-04$ 1.211E-03 1.426E-03 $1.640 \mathrm{E}-0331.854 \mathrm{E}-03$

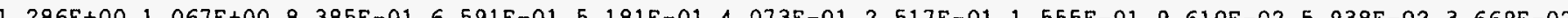
1.538 7.

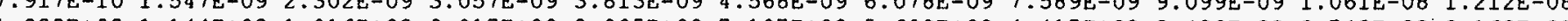

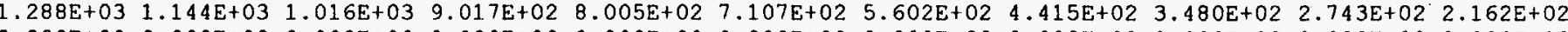

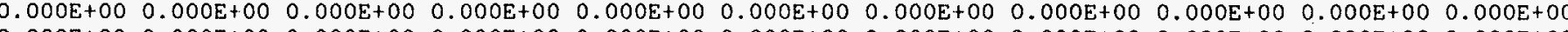
$\begin{array}{lllllllllll}0.000 E+00 & 0.000 E+00 & 0.000 E+00 & 0.000 E+00 & 0.000 E+00 & 0.000 E+00 & 0.000 E+00 & 0.000 E+00 & 0.000 E+00 & 0.000 E+00 & 0.000 E+00\end{array}$ $\begin{array}{lllllllllllllll}0.000 E+00 & 0.000 E+00 & 0.000 E+00 & 0.000 E+00 & 0.000 E+00 & 0.000 E+00 & 0.000 E+00 & 0.000 E+00 & 0.000 E+00 & 0.000 E+00 & 0.000 E+00\end{array}$ 
Appendix G: Sample Output Files From PostoRG

Intentionally Left Blank

G-14 
S. Chakraborti

SAIC

2109 Air Park Road SE

Albuquerque, NM 87106

P. Drez

8816 Cherry Hills Road NE

Albuquerque, NM 87111

Tech Reps Inc. (2)

Attn: M. Minahan

L. Robledo

5000 Marble NE, Suite 222

Albuquerque, NM 87110

\section{Internal}

$\underline{\text { MS }} \quad$ Org.

$1175 \quad 9364 \quad$ J. Liscum-Powell

$05715914 \quad$ T. W. Laub

$13356801 \quad$ M. S. Y. Chu

$13956800 \quad$ L.E. Shephard

$1328 \quad 6848 \quad$ K. Cochrane (4)

$13286848 \quad$ H-N. Jow

$13286848 \quad$ L. C. Sanchez (8)

$13286848 \quad$ B. Schlapper

$13286849 \quad$ J. S. Rath

$13996850 \quad$ M. Brady

$1326 \quad 6851 \quad$ R. Aguilar

13266851 G. E. Barr

$13286849 \quad$ F. J. Davis

$\begin{array}{lll}0734 & 6472 & \text { R. D. Waters }\end{array}$

$11459363 \quad$ P. J. Griffin

11459363 G. Harms

11759364 C. A. Ottinger

$1330 \quad 6811 \quad$ K. Hart (2)

$1330 \quad 4415 \quad$ NWM Library (2)

9018 8940-2 Central Technical Files

08994916 Technical Library (5)

061912690 Review and Approval Desk (2),

For DOE/OSTI 
Intentionally Left Blank 